FI
45 


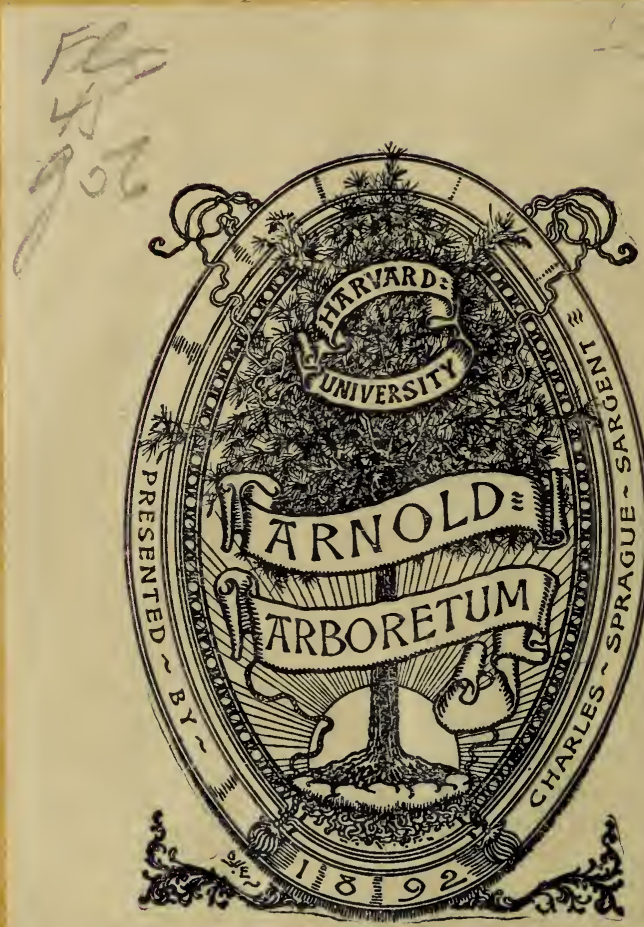


All' Itustre, Aaturalejta - Con "Doarso de Bella - froternem

R. Gouron: 



\section{Divisio I.}

\section{Protihallogamae.}

Tutte le famiglie appartenenti a questa divisione, fatta sinora eccezione delle Isoëteae, sono rappresentate nella nostra flora. Il modo però della loro diffusione e distribuzione è subordinato alle abitudini delle varie forme, e per necessità al complesso delle condizioni esterne ed interne che caratterizzano le varie stazioni. Vediamo pertanto le Rhizocarpeae confinate nella regione della pianura, od appena estendersi alle sponde del Benaco ed al piede dei contrafforti che segnano l'estremo limite delle nostre prealpi; mentre in quella vece troviamo le Equisetaceae, Filices, Lycopodiaceae sparse sopra la intera superficie della Provincia, dalla ima pianura alle valli ed alle vette alpine.

Ordo I.

Equisetaceae.

L' unico genere che compone questa famiglia è rappresentato nella nostra flora da quasi tutte le specie italiane. Gli Equiseti amano in generale i luoghi umidi e sabbiosi, le paludi, le sponde dei fossati, le rive dei fiumi e dei torrenti; qualche volta però mi venne dato di incontrarli in luoghi pietrosi, aridi e secchi. Talune specie salgono dal piano alle regioni elevate dei monti: l'Equisetum variegatum Schl. invece, da questi, seguendo il corso dei torrenti alpini e dei fiumi, si avanza nel cuore della pianura.

\section{Gen. unicum. - EQUisetum.}

\section{Sectio 1. - Equisetum Tournef., Milde.}

1. Equisetum arvense $L . s p . p l$. $1516 ;$ Pollin. viaggio al lago di Garda e al Monte Baldo pag. 16, 84, 118; fl. veron. III, p. 259, et herb.!; Francesco Fontana cat. dei veg. spont. di Lazise p. 29; E. arvense longioribus setis Segu. pl. veron. I, p. 105, n. 4, et cat. plant. quae in agr. veron. 
reper. p. 39; Moren. herb.! ; Coda di cavallo Pona Monte Baldo p. 144. - Vernacolo: Sajola!, Coa de caval, Seola. Come osservai nella Nota, che serve di prefazione al mio Erbario forestale veronese, avendo un giorno domandato ad un villico, quale nome desse alla forma sterile più comune dello $E$. arvense, $\mathrm{mi}$ rispose che la chiamava Pino!

$\beta$ serotinum $F$. W. Meyer chlor. Hanov. p. 666; E. campestre Milde fil. Euro1). etc. p. 217. - . Planta simillima formae - sterili, sive potius transmutatio ejusdem formae, in api- cem caulis spicam sporiferam gerens. '

$\gamma$ nemorosum $A$. $B r$. - Forma sterilis: caule valido - 2-5 dc. m. alto, inferne nudo, pallido; ramis patentissimis. .

o decumbens Meyer. - " Forma sterilis : caule coespitoso, decumbente, e basi ramoso.

$\varepsilon$ varium Milde. - - Forma sterilis: caulis tenuis, erectus, - simplex vel parce ramulosus, in internodiis latericolor. Goir. in exsicc. apud Soc. helvet. (a. 1880).

$\zeta$ arenicola Goiran in herb.! - - Forma sterilis. Rhizoma - pilosissimum: pilis fuscescentibus. Caulis dense coespito- sus : caudiculis a basi ramosis, arcuatis, solo adpressis, in - orbem expansis.

$\eta$ alpestre Whblrg. - Forma sterilis: caulis humilis, - decumbens, internodiis abbreviatis.

Frequentissimo nei prati, nei campi, nei pascoli, nei boschi, negli argini della provincia, dalla pianura a tutta la regione montana, diventa più raro nella regione alpina e subalpina. Le forme fertili si rinvengono sporificate dalla fine di febbraio a tutto aprile, ed anche in maggio e giugno nelle stazioni più elevate; le forme sterili si mantengono sino a tardo autunno. 2

La var. $\beta$ è rara; io sinora non la ho incontrata; se ne conserva un esemplare nell'Erbario Polliniano, raccolto sicuramente nella provincia, il quale si trova mescolato a diversi campioni fertili e sterili, appartenenti questi ultimi alla forma più comune. Le quattro schede però, che accompagnano gli esemplari, portanti tutte la scritta $E$. arvense, sono andate confuse : quindi mi è impossibile indicare la località nella quale Ciro Pollini raccolse questa pianta, da lui ritenuta per fermo come semplice lusus della normale. - Le varietà $\gamma$ e $\delta$ si incontrano qua e là in tutta la provincia dalla pianura ai monti; riferisco alla varietà $\gamma$ una forma elegantissima, assai frequente nei pressi di Verona, nelle siepi umide ed ombrose, il cui caule a stagione un po' inoltrata, diventa di un 
bel nero carico e lucente. - La var. $\varepsilon$ è stata da me raccolta lungo l'Adige, fra le pietre che formano l'argine del fiume, fuori Porta Pellegrina, ed anche nei campi aliacenti. - I donate dall' Adige. - La var. $\eta$ nel M. Baldo alla Lonza, in Novesina, presso la F'onte di Naole, fra 1300 e 1600 metri di altitudine.

Assieme alla var. decumbens cresce puro una forma sterile, coi cauli semplici e sdraiati, che sembra corrispondere all' $E$. arvense var. myriocladum Gandoger (flora gallica exsicc.!).

it assai difficile, per non dire impossibile, asserire quale pianta o meglio quali piante, il Pona nel suo Monte Baldo descritto intenda significare col suo Coda di cavallo di varie specie: denominazione che evidentemente deve intendersi attribuita a varie forme di questo intricatissimo genere, come l'autore stesso indica colle parole di varie specie aggiunte alla denominazione generale di Coda di Cavallo.

2. Equisetum Telmateja Ehrh. in Ilannöv. Magazin, Stück 18, 1793, p. 287; Sacc. critt. vasc. etc. p. 59; Vis. et Sacc. cat. p. 7; E. fluviatile Auctor. plur. non L.; Bertol. fl. ital. crypt. pars I, p. 9, excl. syn. Pont. et Segu.; E palustre longioribus setis et ramosis Pollin. viag. $p .16,118 ; \mathrm{fl}$. veron. III, p. 260, exel. syn. Pont. et Segu. ut supra, et herl.!; Fontana cat. etc. p. 29; E. palustre longioribus setis Segu. pl. veron. I, p. 101, et cat. pl. 39; Coda di cavallo Pona M. Baldo. p. 114. - Icon.: Matth. Valgr. 1585, 2, p. 10so. - Vernacolo: Setola, Seola, Sajola.

$\beta$ comosum Milde fil. etc. p. 219. - - Forma sterilis:

- caule 4.5 dc.m. alto, dimidia parte inferiore ramis destituto:

" ramis erecto-patentibus. '

$\gamma$ breve Milde fil. etc. p. 219. - Forma sterilis: - caule ${ }^{1 / 2}$ dem. $-2 \mathrm{~d}^{\mathrm{cm}}$. alto, erecto, vaginis approximatis;

- densissime ramoso. ,'

Nei luoghi umidi ed acquitrinosi, e specialmente nel margine dei fossi e dei corsi d'acqua di tutti la provincia, dalla pianura ai monti ove diventa più raro: p. e. presso Vigasio! (e Masè herb.!) ove si mostra gigantesco, nei dintorni di Verona in Campo Marzo! (e Tonini herb.!); presso Legnago! (e Rocchetti herb.!), nei colli presso Avesa nel Vajo del Borago!, in $M$. Baldo presso la Ferrara dove lo ha pure osservato il Pollini (viag. p. 118) etc. Le forme fertili si incontrano sporificate dalla fine di marzo al principio di giugno; le sterili si mantengono, nelle stazioni ombrose e nelle elevate, sino al principio dell' autunno. - La var. $\beta$ è stata da me raccolta pressn Avesa nel Vajo del Borago. - La var. $\gamma$ negli argini presso Caldiero. 2 
Sinora non mi venne dato di incontrare la forma serotinum Milde, coi cauli estivi ed autunnali terminati da una spiga. - La pianta di Pontedera comp. tab. bot. pag. 64 e del Seguier pl.veron. I, p. 104, Equisetum palustre, longioribus setis et ramosis, non deve punto, secondo il mio modo di vedere, ritenersi quale sinonimo dell' $E$. Telmateja, come hanno opinato il Pollini ed il Bertoloni. La descrizione che il Pontedera dà della sua pianta, non mi pare possa lasciare a tale riguardo dubbio alcuno: « Ex * radice candida, quae humi reptat, et plurimis geniculis distincta, caules - multi surgunt, nigricantes, bicubitales, simplices, erecti, enodes, cavi, py* xidatim ad genicula cohaerentes, ex quibus geniculis capillamenta in or" bem circumducta nascuntur, geniculata, in terna, quaterna, quina alia - divisa, quae pariter, ut illis inseruntur, ita quoque partes alterius in se * recjpiunt, et hae alias. Caulium summitati capitula oblonga Asparago, ant - quorundam Amentaceorum arborum julis similia insident. » - Che il Pontedera non intendesse punto parlare dell' $E$. Telmateja, oltrechè dalla diagnosi ora riferita, risulta benanco da quanto soggiunge lo stesso autore; il quale così prosegue: Ex his itaque manifestum est, hoc nostrum di* versum esse tum ab Equiseto palustri longioribus setis cui folia simpli- cia sunt (e che corrisponde per lo appunto all' $E$. Telmateja) tum ab * Equiseto foliis nudo ramoso sive Equiseto tertio Matth. ». Ad ogni modo quale pianta intendesse descrivere il Pontedera, non oserei assolutamente asserire, nella mancanza di esemplari autotipi o per lo meno di una figura: l'Autore dice che questo Équisets non è infrequente nell'Agro veronese, ma non ricordo d'avere mai incontrata alcuna forma che possa riferirsi alla pianta descritta da lni. Nell'Erbario del Moreni sotto la scheda Equisetum palustre longioribus setis et ramosis Pont. comp. 64, pl. ver. I, 104 (sotto la quale con carattere, che sembra di Pollini, sta acritto Equisetum fluviatile $s p . p l$.) si trovano due esemplari appartenenti a due specie affatto distinte: un esemplare infatti sembra possa riferirsi ad una delle tante forme dello $E$. ramosissimum, e l'altro è rappresentato dalla parte superiore di un campione sterile di $\boldsymbol{E}$. arvense. Nello stesso Erbario del Moreni la pianta portante la scheda E. palustre longioribus setis (e sotto con scrittura diversa, forse del Pollini, $E$. fuviatile $L$.) spetta all' $E$. ramosissimum. - L'illustre Milde, nella celebratissima monografia degli Equiseti, fra i sinonimi dell' E. Telmateja, non enuncia punto la pianta del Pontedera e Seguier.

Il Bertoloni fl. ital. crypt. a proposito dell'E. Telmateja, il suo E. fluviatile, a ragione osserva che in questa specie: \& juxta caules ste- riles autumno e rhizomate prodeunt turiones breves, clavaeformes, toti - vaginis nigrescentibus, laciniatis tecti, qui anno sequente transeunt in - caulem fertilem; id bene ostendit figura a Matthiolio data, et ab aliis " imitata. Io ho constatato la verità di questa osservazione nel Vajo del Borago presso Avesa: mi pare pertantı) che il Pollini non sia nel vero chiamando malı la figura del Mattioli, la quale per lo appunto, come osserva il Bertoloni, riproduce tale particolarità. 
3. Equisetum palústre $L$. sp. pl. 1516; Pollin. fl. veron. ill, pag. 261, var. a excl. syn. E. veronense Spreng. et Poll.; Sacc. critt. vasc, etc. p. 59; Vis. et Sacc. cat. p. 7; E. palustre brevioribus setis Segu.pl. veron. I, p. 105. - Vernacolo: Coa de caval!.

$\beta$ nudum Duby bot. gall. p. 535; Milde fil. etc. p. 221. - Caulis validus erectus, nudus.

$\gamma$ tenue Döll. rhein. F'l. 1813, p. 29; Milde fil. etc. p. 221. - Caulis debilis, erectus, nudus licet vage ramosus. ;

$\delta$ arcuatum Milde fil. etc. p. 225. - Caulis a basi ra- mosus, pyramidatus, ramis caulis apicem versus decre- scentibus.

$\varepsilon$ polystachyum. - Ramis spiciferis. , - E. polystachyum Vill. hist. pl. Dauph.; E. veronense Spren!l. pl. min. cogn. pug. II, p. 91; Pollin. hort. et prov. veron. pl. nov. vel min. cogn. p. 27; E palustre var. $\beta$ Poilin. fl. veron. 1II, p. 261 (E. palustre minus polystachyum); Berlol. l. it. crypt.p. 12; E. palustre $\beta$ polystachyum Sacc. critt. vasc. etc. p. 59 ; Vis. et Sacc. cat. p. 7 .

La forma tipica cresce abbondantemente nei luoghi umidi e palustri della intera Provincia veronese, dal piano a tutta la regione montana : nei dintorni di Verona!, lungo le rive del Benaco!, presso Costermano!, nelle valle dell'Adige!, nella valle dell'Alpone!, nel M. Baldo presso la Ferrara!, etc. Le varietz̀ crescono promiscuamente alla specie: la varietà $\beta$ è stata di preferenza da me incontrata lungo l'Adige presso S. Michele, il Casino, Centore, la Mambrotta etc.; la var. $\gamma$ in M. Baldo alla Ferrara. - Si incontra sporificata da aprile ad ottobre. $z^{2}$

L' $E$. veronense spetta all' $E$. palustre come riconosce 10 stesso Pollini, che nella sua Flora lo riporta per lo appunto come sinonimo di questo: pero la frase ramis hexagonis apice spiciferis, dimostra che la pianta in quistione deve più rigorosamente riferirsi alla var. polystachyum; il celebre Milde (fil. etc. p. 225) reca appunto l' $E$. veronense come sinonimo di questa varietà. La var. $\beta$ poi della Flora Polliniana (E. palustre minus polystachyum), stando alla figura indicata dal Pollini (Raji syn. meth. t. $V, f .3$ ), riportata pnre dal Milde, apparterrebbe più propriamente alla forma racemosa dello stesso $E$. polystachyum. - Nell'Erbario Polliniano del resto stanno in uno stesso foglio diversi esemplari da riferirsi tutti all' $\boldsymbol{E}$. palustre, od a forme di questo, ad eccezione di due di essi che spettano evidentemente all' $\boldsymbol{E}$. ramosissimum. Tutti questi esemplari sono accompagnati da quattro schede che portano promiscuamente le denominazioni di $E$. palustre ed $E$. veronense; ed uno degli esemplari avera evidentemente in origine i rami spiciferi. 
4. * Equisetum limosum L. sp. pl. 1517; Pollin. fl. ver. III, p. 261, excl. nonnul. syn.; Vis. et Sacc. cat. p. $\%$.

Secondo il Catalogo dei sigg. Visiani e Saccardo, questa specie cresce nei luoghi palustri del Veronese: io però sinora non ho arvuto il destro di osservarla. Il Pollini pure non indica questa pianta nella nostra regione, e l'esemplare di E. limosum che si trova nel suo erbario proviene dalla Germania. - La pianta del Seguier, Equisetum foliis nudum non ramosum sive junceum, pl. ver. $I, p$. 105, non spetta a parer mio all' $E$. limosum. Il Seguier scrive di averlo trovato nei fossi di Verona a Porta Nuova, a destra di chi entra in città: ma nei fossi che sono a destra come a manca di Porta Nuova, io non ho incontrato l' $E$. limosum, bensì diverse forme appartenenti o all' $E$. Schleicheri, ovvero alla specie seguente, quella segnatamente coi cauli nudi, che potrebbe benissimo essere la pianta del Seguier. Anche nell'Erbario del Moreni la pianta che sta con la denominazione di Seguier spetta all' $E$. ramosissimum. E si noti che il Moreni si vale sempre nella classificazione delle sue piante della nomenclatura e delle frasi del Seguier; e che essendo stato frequentemente compagno a questi nelle erborizzazioni, forse più di ogni altro era in grado di sapere quali piante il Seguier intendesse descrivere. Infine io credo che la pianta dello Seguier e del Moreni sia identica a quella dello Zannichelli portante la stessa denominazione (hist. pl. p. 93, t. 57), la cui figura corrisponde per lo appunto all'esemplare dell' Erbario Moreniano, e che io non esito punto a riferire all' $E$. ramosissimum. A torto pertanto il Pollini reca come sinonimo del suo E. limosum, la pianta dello Zannichelli, tanto più che questi a pag. 93 dice che tale pianta per l'asprezza dei suoi rami serve a levigare i metalli: mentre l' $E$. limosum, come è noto, ha i cauli lisci.

\section{Sectio 2. - Hippochaete Milde.}

5. Equisetum ramosissimum Dest. fl. allant. II, p. 398; Milde fil. etc. p. 234; E. elongatum Willd. sp. pl. V, p. 8; Pollin. herb.!; Sacc. critt. vasc. p. 59; Vis. et Sacc. cat. p. 8; E. procerum Pollin. hort. et prov. veron. pl. nov. vel. min. cogn. p. 28; E. hyemale $\beta$ procerum Pollin. fl. ver. III, p. 26.2; E. palustre longioribus setis Moren. herb!. - Vernacolo: Seola.

$\beta$ virgatum $A l$. $B r$. - Coespitosum: caulis erectus, - scaber, nudus licet vage ramosus. ,

$\gamma$ simplex Döll. - " Caulis simplex, erectus, nudus. , $\delta$ gracile $A l . B r$. - Coespitosum: caulis basi procum- bens vel erectus, scabriusculus, a basi vel in medio ra"mosus." 
$\varepsilon$ altissimum $A l . B r$. - Caulis viridis, scabriusculus, - inferne dense verticillatus, superne nudus vel vage ramo- sus, scandens inter frutices: vaginae amplae, margine an" gusto nigro. '

$\zeta$ polystachyum. - Ramis spiciferis. .

Nei luoghi arenosi, ghiaiosi, umidicci della parte piana della intera provincia, copiosissimo: diventa più raro nei colli e nei monti ove compare qua e là. Io l'ho raccolto od osservato negli argini lungo l'Adige in Campo Marzo, al Pestrino ccc., lungo tutto il corso del fiume in una parola; nei fossi della citti di Verona a Purta Nuova; nella Valle dell'Adige presso Ceraino, Peri, Avio, ecc., nellir Val Pantena, e Val Policella, alle sponde del Benaco, lungo il Mincio ecc. Ise forme citate, cal altre molte che si potrebbero aggiungere, crescono e virono promiscuamente: la $\varepsilon$ si incontra di preferenza nelle siepi, nei canneti, fra i virgulti e qualche volti anche in luoghi aridissimi, p. e. al Bosco MIantico!; la $\zeta$ è piuttosto rara. - Le spighe cominciano a spuntare a mezza primavera: però si può affermare che la pianta si conscrva in vegetazione durante tutto il corso dell'auno, almeno nelle stazioni sufficientemente riparate dai rigori dell' inverno e dalla arsura estiva. 2

L'E. ramosissimum è pianta eminentemente polimorfa : alcune forme si vanno arvicinando all' $\boldsymbol{E}$. hyemale, e taluna, massimamente sul secco, si può di leggieri confondere con quelie corrispondenti all' E. Schleicheri Milde. Gli illustratori delle piante veronesi credo che sieno, secondo ogni probabilita, incorsi in tale equivoco, del resto facilissimo.

L'E. procerum Pollin. spetta sicuramente all' E. ramosissimum, sebbene il Bertoloni (fl. ital. crypt.pars I, p. 15) lo consideri come sinonimo dell'E. hyemale. Il Pollini stesso lo sospettava nella sua Flora: nella quale ritiene bensì l' $E$. procerum come una varietà dell' $E$. hyemale, ma contemporaneamente nelle annotazioni a questa specie ( $f$. ver. III, $p .263$ ) si mostra titubante tra il ritenerlo come specie distinta ovvero come una varietà dell' $E$. elongatum Willd., che è per lo appunto sinonimo del nostro. Anche l'illustre Milde riporta lo E. procerum Pollin. come sinonimo dell' $E$. ramosissimum.

L'E. ramosissimum si trova nell'Erbario Polliniano sotto la scheda E. elongatum, raccolto nei fossi delle fortificazioni, ed al Pestrino, ove cresce copiosamente: e fra le diverse schełe che accompagnano gli esemplari, se ne trova una portante la intitolazione $E$. elongatum con la frase diagnostica della pianta, e sotto aggiuntovi $E$. procerum.

Nell' Erbario Moreniano si trova pure l'E. ramosissimum sotto la scheda $E$. palustre longioribus setis: un esemplare fertile ed intatto, che vi si conserva, non lascia dubbio alcuno. Ed errò chi sotto la scritta del Moreni pose la denominazione $E$. fluviatile: il vero $E$. fluviatile $L$. appartiene all' $E$. limosum, ha la spiga ottusa, non mucronata ccme nella pianta del Moreni. E non posso a meno di esternare il sospetto che alla specie in questione debba pure appartenere la pianta del Seguier, Equi- 
setum palustre longioribus setis et ramosis (pl. ver. I, p. 104, n. 2) : la stazione indicata por questa dall'autore (Veronae secus Athesim) non fa che confermarmi in tale sospetto.

Nelle siepi presso S. Michele, Rivole ecc., ho frequentemente osservato un Equiseto col caule scandente, alto talora sino a due metri, più o meno ramoso. Io non esito punto a riferirlo all' $E$. ramosissimum; e precisamente alla forma corrispondente all' $E$. altissimum $A l$. Br., che mi sembra scolpita dal Cesalpino nel suo $E$. longissimo caule in sepibus scandens (de pl. lib. 16, p. 598).

La figura del Nattioli, F. tertium (ed. Valgrisi p. 1028) non spetta punto all' $E$. hyemale, come la ritiene il Pollini, ma bensì al nostro: al quale appartiene benanco la pianta del Micheli, $E$. altissimum scandens asperum monostachyon et polystachyon setis plerumque ramosis et praelongis (cat. pl. hort. $f l . p$. 25) riferita dall'autore alla citata figura del Mattioli. A torto perianto il Pollini ( $f$. ver. III, p. 260), riporta la pianta del Mattioli quale sinonimo del suo E. sylvaticum.

6. Equisetum hyemale $L . s p . p l$. 1517 ; Pollin. viag. p. 16, 84, 11i; fl. veron. III, p. 26.2, excl. syn. et icon. Matth. et var. $\beta$ (E. procerum); Bertol. fl. it. crypt. p. 15, excl. syn. E. proceri Pollini; Sacc. critt. vasc. etc. p. 59; Vis. et Sacc. cat. p. 20. - Vernacolo: Scola.

B Schleicheri Milde fil. ctc. p. 244; Goir. appunti bot. in Cron. Alp. p. 68. - - Planta polymorpha; caulis nudus vel a vage ramosus, plerumque sordide viridis: vaginae saepe, " in speciminibus nostris, concolores, pallide virides, elon" gatae, ampliatae: dentes vaginarum plus minusve per- sistentes, appendice membranacea subulata instructi. Ab - E. ramosissimo non absimile. .

La specie cresce nel M. Baldo nelle selve che stanno intorno al Pian della Cenere! (1000 m.) (Pollin. herb.!), nei Lessini presso Revolto $(994 \mathrm{~m}$. ove venne raccolto da Caro Massalongo. La var. $\beta$ si incontra nei monti Lessini presso Revolto!, nella Valle dell'Adige presso Rivole!, lungo l'Adige presso Verona ecc. Non è molto l' ho raccolto con l'amico corte Giulio Giusti lungo il Tione presso Guastalla nell'Agro Veronese. - Dalla primavera a tardissimo autunno: frequentemente, come ho potuto verificare per le piante che crescono in M. Baldo, nei pressi del Pian della Cenere, si mantiene durante l' inverno, arendo nel giugno scorso raccolte le piante appartenenti sicuramente all'anno precedente. 2

Gli esemplari che stanno nell'Erbario Polliniano, ed in quelli di A. Massalongo e di Carlo Tonini sotto la denominazione di $\mathbb{E}$. hyemale, fatta eccezione di quelli raccolti presso il Pian della Cenere, spettano o all' $E$. ramosissimum, ovvero a forme prossime all' $E$. Schleicheri. - 
L'E. Schleicheri da me raccolto nel Veronese concorda pienamente coi campioni ungheresi del dottor Tausccher e con quelli dell' erb. critt. ital. n. 1301, coi quali ho istituito il confronto.

7. Equisetum variegatum Schleich. cat. pl. helv. p. 27; Vis. et Sacc. cat. p. 7; Goir. pl. ver. nov. vel min. cogn. etc. cent. I, p. 17, et app. bot. etc. p. 68; E. nudum minus variegatum Basileense C. B. prodr. p. 21, n. 21; Moren. herl.!

$\beta$ anceps Milde fil. etc. p. 248. - . Pusillum: caulis te- nuissimus. .

Lungo i rigagnoli ed i torrenti montani, scendendo insino alla pianura: in Campion! (1200 m.), alle Acque negre! ecc. nel M. Baldo; alla Giazza! (800 m.), Revolto! (994 m.) ecc. nei Lessini. Si trova pure in Val d'Adige presso Dolcè, Ceraino ecc., nonchè presso Verona in luoghi acquitrinosi oltre il porto di S. Pancrazio!, a S. Michele!, Casino!, Giarone!, Centore! ecc.; nel complesso fra $30 \mathrm{~m}$. e $1600 \mathrm{~m}$. di altitudine. La var. $\beta$ ì stata primieramente segualata dall'illustre Trevisan nei M. Lessini : io la ho osservata nel M. Campostrin al dissopra di Giazza, e nel M. Baldo alle Acque negre. - Nell' estate e nell'autunno uelle stazioni elevate, durante quasi tutto l'anno nella pianura. 2.

Secondo ogni probabilità questa pianta è stata confusa con qualche forma spettante all' $E$. ramosissimum, al quale è vicinissima. La pianta che sta nell' Erbario del Moreni sotto la denominazione sopra riportata spetta sicuramente all' $E$. variegatum. - Gli esemplari veronesi di questa pianta, da me raccolti nello vicinanze della città e nella Valle dell' Adige, concordano con quelli del Modenese e di Francia: la pianta del Casino e Giarone è la esatta riproduzione di quella distribuita dalla Société helvétique pour l'ćchange des plantes, e di altre austriache favoritemi dal dottor E. Hackel.

Non mi è stato dato sinora di incontrare l' E. sylvaticum L. (Pollin. fl. veron. III, p. 260, excl. syn. Michelii): siccome però questa bella specie cresce nei luoghi selvatici ed ombrosi del Vicentino e del Trentino, così non reputo improbabile che la stessa possa rinvenirsi presso di noi; in ogni caso sarebbe da rintracciarsi nelle stazioni subalpine dei monti confinanti con queste due provincie. Nell' Erbario del Pollini si trova l' E. sylvaticum, ma senza designazione di località, proveniente secondo ogni probabilità dai monti Trentini, nei quali il chiarissimo autore della Flora Veronese dichiara di aver incontrato la specie in quistione. - Nell' Erbario del Moreni l' $E$. sylvaticum si trova sotto la denominazione di $E$. palustre tenuissimis et longissimis setis: ed in vicinanza di questo si trova una forma sterile di Equiseto con la scritta $E$. sylvaticum tenuissimis setis, che potrebbe forse riferirsi all' E. capillare Hoff m. - Molto probabilmente potrà pure rinvenirsi nella nostra regione l' $E$. pratense Ehrh. 
In generale appo noi le bestie da pascolo rifuggono dagli Equiseti, e se devo prestar fede a quanto venne narrato da una effemeride cittadina, or fanno pochi anni, nei Lessini diversi vaccini perirono dopo essersene cibati. Noterò come un fatto degno di osservazione, che nei monti accade rarissimamente di vedere $\mathrm{i}$ vaccini, le capre nate nelli stessi ecc., cibarsi delle piante velenose quivi crescenti: ho osservato frequentemente casi di meteorizzazione, mai di vero avvelenamento; il quale invece non di rado si verifica per gli animali nati e cresciuti nella pianura, e che, come è costume, salgono nei monti a passarvi la stagione estiva.

\section{Ordo II.}

\section{RHizocarpeae.}

Le Rhizocarpeae vivono nei luoghi umidi, nelle paludi, nei fossati ad acque stagnanti ovvero a lento corso, nelle risaie; nella provincia nostra, come si è detto, sono confinate nella regione della pianura; si spingono appena sino alle sponde più meridionali del Benaco (Marsilia quadrifolia, Salvinia natans), ed ai piedi delle ultime diramazioni delle nostre prealpi, p. e. Monteforte (Salvinia natans). Manca sinora appo noi il genere Pilularia; che pure nella finitima provincia di Brescia è rappresentato dalla Pilularia globulifera (Zersi).

\section{Subordo I. - Marsiliaceae.}

Gen. I. - Marsilia.

8. Mar'silia quadrifolia $L . s p . p l$. 1503; Pollin. viag. p. 17; fl. veron. III, p. 295, et herb.!; Sacc. critt. vasc. etc. p. 60; Vis. et Sacc. cat. p. 7; Lenticula palustris quadrifolia Segu. pl. veron. I, p. 77; Moren. herb.! - Vernacolo: Erba ranina, Pavarina, Trifoglio d'acqua.

Qua e là nelle risaie $\theta$ nei fossati poco profondi e poco correnti della pianura veronese: Peschiera! (e Pollini), Legnago!, Asalogna!, Arcole! ecc. - Estate ed autunno: è sporificata in luglio-ottobre. 2 Noto che la forma sterile è presso di noi più frequente della fertile.

$$
\text { Subordo II. - Salviniaceae. }
$$$$
\text { Gen. II. - Salvinia. }
$$

9. Salvinia natans All. fl.ped. 1I, 1; Pollin. ind. delle piante dei colli Eug. pag. 24; viag. etc. p. 18; fl. veron. III, p. 294; 
Sacc. critt. vasc. ctc. p. 60; Vis. et Sacc. cat.p. 7 ; Marsilea natans L. sp. pl. 1562; Salvinia vulgaris acquis innatans, foliis subrotundis punctatis laete virentibus Scgu. $p l$. ve. ron. III, p. 52; Moren. herb.! - Vernacolo: Pavarina.

Nelle risaie e nei fossati poco corenti della pianura veronese frequentissima e copiosissima; in alcuni fossi, ove vive promiscuamente alle Lemne, le acque ne sono letteralmente coperte: Peschiera! (e Pollin.), Caldiero!, Monteforte!, S. Bonifacio!, Arcole!, Legnago!, Asalogna!, Vigasio!, Bovolone! ecc. Dalla primavera a tardo autunno: presso Caldiero, stante forse la temperatura delle acque che alimentano i fossati e provengono dalle sorgenti termali, si mautiene in regetazione anche ad inoltrato inverno: è sporificata in estate ed autunno. $z-$ Anche nella Salvinia natans, il numero degli individui sterili è di gran lunga superiore a quello dei fertili.

Ordo III.

\section{LyCOPODIACEAE.}

Le Lycopodiaceae nostre amano in generale le stazioni più o meno elevate dei monti: la sola Sclaginclla, helvetica scende dalla regione alpina ove è rara, infino alla pianura ove cresce copiosamente. Il Lycopodium inundatum, trovato una volta da Ciro Pollini (?) presso Bovolone, è scomparso.

\section{Subordo I. - Lycopodieae.}

Gen. I. - Lycopodium.

\section{Sectio 1. - Selago.}

10. Lycopodium Selago L. sv. pl. 1565 ; Pollin. viag.p. 113 ; fl. veron. 1II, p. 268, et herb!; Sacc. critt. vasc. etc. p. 60; Selago vulgaris abietis rubrae facie Moren. herb.! Vernacolo: Erba piocina, Erba per i pioci.

$\beta$ laxum Desv. - Folia modice longa integerrima - erecto-incurvata (Milde).

Nei luoghi selvatici del M. Baldo, dei Lessini e dei monti che ne dipendono, dalla regione montana in su. Nel M. Baldo in Valle delli Ossi!, Val grande!, Valle delle pietre!, Cime dell'Artillon!, Campion! (e Pollini). Acque negre (e Pollini), Lavacci ecc. Nei Lessini alla Sega!, Revolto!, Valle dei Ronchi! ecc. ; in Campobruno!; nei pascoli del M. Bolea (A. Massalongo in herb. Da Campo!), - La var. $\beta$ è indicata nei Lessini dal 
chiaris. Trevisan. Da giugno ad ottobre: io '1' ho incontrato sporificato al finire dell'estate e nell'autunno. $2^{\circ}$

Le contadine Cadorine, che vengono a smerciare sul nostro mercato lavori in legno destinati a diversi usi, vendono pure mazzettini secchi di L. Selago: questa pianta finamente polverizzata, mescolata al sapone, forma un cosmetico o pomata destinata ad uccidere gli insetti del cupo.

Una forna da me raccolta in M. Baldo alle Acque negre, e nel Trentino alle sponde del lago di Tovel in Valle di Non, si allontana assai dalla forma comune per $i$ cauli più lunghi e quasi repenti.

\section{Sectio 2. - Lepidotis.}

11. Lycopodium annotinum $L . s p . p l$. 1566; Pollin. fl. veron. I1I, p. 165, et herb.! ; Sacc. critt. vasc. etc. p. 61; Vis. et Sacc. cat. p. 8. - Vernacolo: Erba da scoli!, Scoli!.

Nei luoghi selvatici alpini e subalpini del M. Baldo e dei Lessini, e nelle stesse località del precedente, col quale lo ho sempre trovato crescere promiscuamente. - Da luglio a tardissimo autunno: lo ho raccolto sporificato in ottobre 2 .

I nostri pastori si servono di questa pianta come di filtro per colare il latte appena smunto: di qui l'origine del nome volgare.

12. * Lycopodium inundatum $L . s p . p l .1565$; Pollin. $f l$. ver. III, p. 265 et herb. quoad plantam Norvegicam!; Sacc. critt. vasc. etc. p. 61.

Il Pollini dice di aver incontrato il $L$. inundatum una sola volta nei luoghi palustri presso Bovolone; forse si tratta di una pianta scomparsa dalla nostra flora, più probabilmente di un equivoco. Due esemplari di $L$. inundatum esistenti nell' Erbario del Pollini sono della Norvegia; la pianta che si trova in questo stesso Erbario sotto la scheda L. inundatum, proveniente dal Veronese, ma senza indicazione precisa di località, è affitto diversa da questo, trattandosi in essa puramente e semplicemente di un' Alga. Nello stesso equivoco è pure incorso il Da Campo, il quale modellò il suo Erbario su quello del Pollini.

13. Lycopodium clavatum $L . s p . p l .1564 ;$ Pollin. viag. p. 113; fl. veron. III, p. 261; Sacc. critt. vasc. etc. p. 61; Vis. et Sacc. catal. p. 8; Lycopodium Segu. pl. veron. I, p. 64. - Vernacolo: Erba da Scoli!, Scoli!

Nei M. Lessini (Seguier), in Valle dei Ronchi (Pollin. herb!.); Giazza, Zeola, Maléra, Velo, (A. Massal. herb.) ; Selva di Progno (A. Massal. in herb. De Campo!); in M. Baldo a Campion ed alle Acque negre assieme 
al $I$. annotinum e $L$. Selago coi quali cresce promiscuarnennte (Pollin. herb.!) ecc.: in complesso fra $860^{\circ} \mathrm{m}$. e $1800 \mathrm{~m}$, di altitudine. - Luglio, ottobre 20 .

I pastori lo adoperano come il $J_{\text {. }}$ annotinum per colare il latte.

14. Lycopodium complanatum $L . s p . p l$. 1567; Pollin. viag. p. 13; fl. ver. III, p. 26j, et herb.!; Bertol. fl. it. crypt. pars. I, p. 21; Sacc. critt. vasc. etc. p. 61; Vis. et Sacc. cat. etc. p. 8.

Nel M. Baldo a Campion, alle Acque negre, e nelle selve prospicienti il Benaco (Pollin. herb.!); e nei Lessini presso Selva di Progno (A. Massal. in herb. Da Campo!), Rovere di velo (A. Massul. herb.!); nel M. Bolca (A. Massal. herb.!). - Luglio-ottobre. 2

Credo io pure che il $L$. alpinum L. sp. pl. 1567 sia una semplice forma del $L$. complanatum, dipendente dalla stazione; tra gli esemplari di $L$. complanatum che si trovano nelle reliquie Massalongiane, uno di

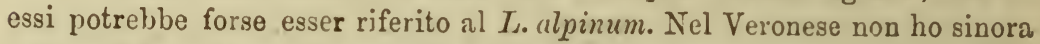
trovato il $L$. alpinum : li esemplari che si trovano sotto questo nome nell'Erbario Polliniano (senza designazione di località) meritano speciale considerazione perchè le spighe sono bifide.

\section{Subordo II. - Selaginelleae.}

\section{Gen. II. - Selaginella.}

Sectio 1. - Homotropae Al. Br., Milde.

15. Selaginella spinulosa Al. Br. ap. Doell. rhein. Flora p. 38; Lycopodium selaginoides L. sp. pl. 1565; Pollin. viag. p. 102; fl. veron. III, p. 266, et herb.!; Bertol. fl. it. crypt. pars 1, p. 20; Selaginella spinosa Beauv.; Sacc. critt. vasc. etc. p. 61; Vis. et Sacc. cat. p. 8; Selaginoides foliis spinosis Segu. pl. veron. III (Suppl.), p. 51; Moren. herb.!

Nei pascoli elevatissimi e nelle vallate alpine: nel M. Baldo a $\mathrm{Co}_{0}$ stabella! (2060), Valle delle pietre!, Valle degli Ossi!, Altissimo di Nago! (2117 m.); nei Lessini e nei monti adiacenti p. e. M. Posta! (2198 m.) e Campobruno! (1800 m.) ecc. Scende pure qualche volta nella regione montana: nell'Erbario Da Campo si conservano esemplari raccolti dal sempre compianto Abramo Massalongo nei pascoli del $M$. Bolca $(945 \mathrm{~m}$.), nel M. Baldo non la ho mai trovata al disotto dei $1200^{\mathrm{m}}$. - Giugno-ottobre. 2

Il Moreni avrebbe una volta raccolta la $S$. spinulosa nei fossi di Verona fuori porta $S$. Zeno: si tratta evidentemente di un equivoco, perchè questa Licopodiacea è pianta essenzialmente della regione alpina e subalpina. 


\section{Sectio 2. - Dichotropae Al. Br., Milde.}

16. Selaginella helvetica Link fil. hort. berol. p. 159; Sacc. critt. vasc. etc. p. 62; Vis. et Sacc. catal. etc. p. 8; Lycopodium helveticum L. sp. pl. 1568; Pollin. viag. etc. p. 128; fl. veron. III, p. 266, et herb.!; Bertol. fl. crypt. pars I, p. 25 ; Lycopodioides Segu. pl. veron. I, p. 65 ; Lycopodioides denticulatum pulchrum, repens, spicis pediculis insidentibus Moren. herb.! - Vernacolo: Sirizinella!

Dalla pianura, ove è abbondantissima, alla regione alpina ove si incontra raramente. Presso Legnago! (Rocchetti herb.!); nei luoghi ombrosi o nelle siepi presso Verona non che nei fossi della città stessa presso S. Zeno! (Pollin. herb.!), in Campo Marzo (Tonini), nei luogni ghiaiosi lungo l'Adige! (Pollin. herb.!, e De Bracht), al Pestrino! (Pollin. herb.!), in tutta la Valle dell' Adige!, nei Lessini presso Velo (A. Massal. herb.!) ecc., nel M. Baldo presso la Corona! (776 m.), sopra la Vat fredda (1200-1600 m.) ecc. Durante tutto l'anno a seconda delle condizioni delle varie stazioni. 2

Frequentemente, come osserva il Milde, i cauli di questa Selaginella assumono un color rosso mattone ed anche sanguigno; al Pestrino lungo l'Adige, a Brentino alle falde di M. Baldo, ed in questo istesso monte nei pascoli di Valfredda, ho verificato questa particolarità nella intera pianta: dichiaro perd che cid ho notato sempre di primavera e sopra esemplari che probabilmente avevano svernato. Il chiarissimo Milde dice pure di questa pianta: nusquam in regione alpina vidi ut autores referunt; io per parte mia la ho trovata in M. Baldo fra i $1200 \mathrm{~m}$. $\theta 1600 \mathrm{~m}$., $\theta$ ad una altitudine ancora maggiore nel Trentino in $\mathrm{Val}$ di Non, nei monti che sono sopra S. Felice, e precisamente nel M. Luch. Nel M. Baldo, nella Valle del Bastion, ho visto nell'anno in corso la S.helvetica crescere promiscuamente alla S. spinulosa.

In questi ultimi anni questa elegantissima Licopodiacea è stata introdotta nelle sale quale pianta di ornamento.

\section{Ordo IV.}

\section{Filices.}

(Milde).

Dei generi italiani appartenenti a questa bella famiglia, nella nostra flora non sono rappresentati finora i gen. Hymenophyllum, Gymnogramme, Allosorus, Onoclea (Struthiopteris germanica W.), Woodsia: appena nomino il gen. Woodwardia 
perchè l'unica specie che lo costituisce, Woodwardia radicans Sw. venne esclusivamente raccolta nelle parti meridionali della Penisola, e nelle isole d' Ischia e di Sicilia. Nella no. stra Provincia la distribuzione topografica delle Felci dipende naturalmente dalle condizioni del suolo, dalla esposizione e dalla altitudine. Mentre taluna specie non oltrepassa la regione collina o tutto al più la montana, altre invece dalla pianura, ovvero dai colli, penetrano nei più cupi recessi dei monti; alcune sono caratteristiche della zona alpina, subalpina e montana; una, Aspidium Thelypteris Sw., si trova confinata nei paduli e nei fossati della pianura, ed appena si spinge alle sponde del Benaco, ed al piede degli ultimi contrafforti delle nostre alpi al confine orientale della Provincia.

\section{Subordo I. - Polypodiaceae.}

Tribus 1. - Polypodiaceae.

Gen. I. - Polypodium.

17. Polypodium vulgare $L . s p$. $p l$. 1514 ; Segu. pl. veron. I, p. 72; Moren. herb.!; Pollin. viag. etc. p. 89; fl. veron. III, p. 273!; herb.!; Fr. Fiont. cat.p. 32 ; Bertol. fl. it. crypt. pars I, p. 11; Vis. et Sacc. cat. p. 9; Polipodio Calc. viag. p. 8. Vernacolo: Polipodio, Felese, Felese dolsa, Regolissia.

- Stirps summopere varians: e planitie ascendit ad regionem sub* alpinam; in alpina regione quoque occurrit, sed rarius. Mibi peragranti, * sequentes obviam fuere formae vel insigniores, vel magis frequentes: "

a commune" Milde, filices Europxe etc. p. 18. - a Sugmentis oblongis, apice subito acuto, ibique minute serulatis. - Forma frequentissima; nella pianura : presso Verona nel Bos:o Mantico!, presso Legnago (Rocchetti herb.!) ecc.; nella regione collina: nelle convalli presso Avesa!, in Val Pantena (Tonini!), a Ponti presso Peschiera (Da Campo herb.!) ecc.; e nei monti: nel Pastello!, nel Baldo!, nei Lessini!, e specialmente nel Bolca!.

$\beta$ angustum Milde, loc. cit. - * Lamina angusta, longa, longe pe* tiolata: segmentis oblongis, apice subito acuto, ibique minute serru- latis. - Nel Baldo e nei monti prossimi o adiacenti ad esso, ma meno frequente della forma precedente.

$\gamma$ rotundatum Milde, loc. cit. - \& Segmentis late rotundatis, vix ser* rulatis. , - Si incontra frequentemente assieme alla var. $\alpha$.

$\delta$ serratum $W .-$ Polypodium serratum W. sp.pl. V, p. 173; P. vulgare $\beta$ serratum Vis. et Sacc. cat. p. 9 ; Goir. appunt. bot. in Cron. 
alp. p. 68; exsicc. Goir. ap. Société helv. pour l'échange des plantes. "Frondibus (in speciminibus veronensibus) 40 cent. longis : lamina $25 \mathrm{~cm}$. - longa, $19 \mathrm{~cm}$. lata : segmentis longe acuminatis et toto margine grosse a serrato-dentatis. Pulcherrima forma! rara tamen in ditione nostra. In Val Pantena nelle rupi sopra Orè (200 m.)!, nel Vajo della Pernise alle falde dei Lessini!, nel Vajo di Squaranto!, e nel M. Baldo al Santuario della Corona (776 m.)!. Ad Orè numerosissini e lussureggianti esemplari ricoprono letteralmente in alcuni punti, le rupi ombrose ed umide che guardano verso settentrione e levante; le frondi, nella pianta fresca, frequentemente appaiono con una vaghissima tinta glauca. Ho raccolto nei Lessini e nel Baldo forme intermediario che segnano il passaggio dalla var. serratum alla forma tipica.

$\varepsilon$ pumilum Goir. et Tonini; Goir. spec. morph. veg. p. 12; lapp. bot. etc. p. 68. - Frondibus 6-10 cent. altis: lamina vix attenuata - 5-7 cent. longa, 5-5,5 lata: segmentis lineari-oblongis, obtusiusculis * brevissime apiculatis, e medio serrulatis: segmento supremo lanceolato, \& acuminato. Forma pusilla praecedentis. - Promiscuamente alla forma precedente che rappresenta, si può dire, in miniatura.

$\zeta$ auritum W.; Goir. app. bot. etc. p. 69. - C Segmentis infimis, a basi superiore, vel inferiore, vel utrinque anriculatis vel lobatis. »Nel Zlosco Mantico!; nel Vajo del Borago! e Pissacavra! presso Avesa; in Quinto!; al Cerro! ecc. al piedo del tronco dei Castagni, mescolato alla forma normale, a Ponti presso Peschiera (herb! Da Campo senza (ieterminazione). - Anzichè una varietà, nel senso che generalmente si s:rol dare a questa parola, io considero questa forma come una semplice anamorfosi; come una deviazione ciò̀ dalla tipica. La quale deviazione fi.equentemente si esagera, talchè come osserva il Milde (loc. cit.) saepissime in formas monstrosas exit; mentre altra volta trovansi forme intermediarie che .passano gradatamente alla normale. Ho raccolto p. e. presso Alcenago, in Val Pantena, un esemplare nel quale i due segmenti infimi, nella base superiore presentano appena due leggerissime protuberanze semicircolari; un altro in quella vece, raccolto da me in vicinanza del Cerro, ha i due segmenti infimi laciniati irregolarmente nella base inferiore, e le lacinis hanno una lunghezza compresa fra 12 e $15 \mathrm{~mm}$.

Il $P$. vulgare, colle sue numerose varietà, si mantiene in vegetazione durante tutto l'anno, almeno nei luoghi riparati o dalla eccessiva arsura dell'estate, o dai soverchi rigori dell'inverno. In questi ultimi anni ho visto frequentemento il $P$. vulgare coltivato in vasi per ornamento delle sale. 2

Gen. II. - Adiantum.

18. Adiantum Capillus-Veneris L. sp. pl. 1558; Pollin. f. veron. III, p. 293, et herb.! ; Franc. Font. cat. etc. p. 27 ; Beriol. fl. it. crypt. pars I, p. 97; Vis. et Sacc. cat. etc. p. 13; Adiantum foliis Coriandri Segu. veron. I, p. 73. - Vernacolo: Capilvenere, Capiler, Capelvenere. 
Nelle rupi o nei luoghi umidi dei colli e dei monti più bassi : nella citta stessa di Verona nelle pareti dei pozzi!; lıngo il Benaco presso Iazise, Malcesine!, Cassone! Castelletto! ecc.; presso Avesa al Tagliaferro!; nelle valli dell'Adige!, Policella!, Pantena!, di Illasi nella quale il Da Campo (herb.!) la raccolse nel luggo detto le Fontanine presso Tregnago. - Dalla primavera al principiar dell'inverno. 2

Anche il Capelvenere viene da alcuni anni coltivato in vasi quale pianta d'ornamento.

\section{Gen. III. - Cheiranthes.}

19. Cheilanthes Szovitsii Fisch. et Meyer in Hohenacker plant. Prov. Talysch, et Bull. soc. Mosc. $11 I$ (1838), p. 211, et Bull. soc. Mosc. VI (1833), p. 260; Milde fil. ctc. p. 33; Comp. fl. it. etc. p. 22; Cheilanthes fimbriata Vis. fl. dalm. p. 12; Acrostichum microphyllum Bertol. fl. ital. crypt. pars 1 , p. 35; Oeosporangium Szovitsii Vis. in Atti R. I. Ven. XII, serie terza, p. 656-61; Vis. et Sacc. cat. ctc. p. 13; Cheilanthes persica Trevisan syll. sporophyt. Ital. in Atti della Soc. Ital. di scienz. nat. XVII, p. 233. - Icon. Hool. Spec. fil. II, tab. $91 \mathrm{~B}$; Bertol. miscellan. botan. XVIII (1858), tab. 1; Vis. fl. dalm. tab. 1, fog. 1, e atti R. I. Ven. etc. tab. X et XI.

Rarissima. $2-$ - Questa interessantissima felce,rarissima per l'Italia ove venne primieramente scoperta nel M. Mauro dal Tassinari, è stata raccolta dal sempre compianto Carlo Tonini nel M. Baldo. Il Tonini mando tutti i suoi esemplari all'illustre Antonio Bertoloni, il quale accenna la provenienza Veronese di questa pianta nella sua fl. ital. crypt. loc. cit.: questi esemplari si conservano tuttora nello storico Erbario del Bertoloni in Bologna, come il figlio di lui prof. Giuseppe mi dichiarava per lettera, qualche giorno prima venisse rapito alla scienza ed agli amici. Fatalmente questa pianta manca nell'Erbario del Tonini; nè questi ripetutamente interrogato da me, potè mai ricordare la località del monte ove la rinvenne; e malgrado le più diligenti e minuziose ricerche, finora non mi venne dato di imbattermi in essa. Nelle reliquie botaniche del celeberrimo Abrámo Massalongo, il figlio Caro ha trovato in uno stesso foglio, indeterminati e mescolati assieme, esemplari di Ch. Szovitsii e di C'h. fra grans: mancando però ogni scheda, non sò che dire sulla loro provenienza; però io ritengo che gli esemplari del prof. A. Massalongo non sieno punto veronesi, ma che, secondo ogni probabilità, provengano dalla Dalmazia.

\section{Gen. IV. - Pteris.}

20. Pteris aquilina $L . s p . p l$. 15.33 ; Pollin. fl. veron. III p. 291, et herb.! ; Franc. Font. cat. p. 33; Bertol. fl. ital. 
crypt. pars I, p. 89; $\nabla$ is. et Sacc. cat. etc. p. 12; Felce femina Calc. viag. di M. B. p. 11; Pona M. B. p. 230; Filix ramosa major pinnulis obtusis non dentatis Segu. pl. veron. I, p. 60; Moren. herb.!. - Vernacolo: Felese, Felze, Felese dell'Aquila!, e presso Badia Calavena, 'Fòldare: nome, come si dirà, comune ad altre specie di Felci.

Dalla pianura ai colli ed ai monti. 2 - Volgarissima nelle siepi, nei boschi e negli stessi luoghi coltivati che stanno ai margini di questi; diventa più rara nella regione alpina. Comincia a vegetare dalla fine di marzo o dal principio di aprile, nei luoghi bassi ed aprici, e dura sino in ottobre ed anche novembre. Nei boschetti ombrosi dei colli ho osservato individui che superavano l'altezza di due metri; mentre nei luoghi più aridi e secchi altri toccavano appena il decimetro. - Nei nostri monti questa felce serve nelle stalle per fare il letto al bestiame; se ne avvolge il ghiaccio che si trasporta in città, i formaggi ecc.

La Pteris aquilina raramente, in questa nostra zona, si incontra con le frondi fertili; e lo stesso posso affermare di avere osservato in altre regioni da me visitate. Nel Veronese ho raccolto individui sporificati due sole volte: in una siepe nel M. Bolca, ed in un mucchio di pietre fra pruni e rovi nei M. Lessini presso Cerro. Tutte le felci, come è noto, presentano delle forme sterili : ora le numerose osservazioni da me fatte mi inducono a ritenere, che le forme sterili si incontrano per lo più negli individui che offrono una maggiore esuberanza nel complesso della vegetazione. Tutti i generi appartenenti alle felci nostrane hanno somministrato delle prove in favore di questa proposizione: si direbbe quasi che nelle forme sterili il lavoro, che chiamerò di sporificazione, si è trasformato in lavoro semplicemente vegetativo.

21. Pteris cretica L. mant. 130; Pollin. fl. veron. III, p. 291, in notis; Bertol. fl. ital. crypt. pars 1, p. 88; Vis. et Sacc. cat. etc. p. 276 .

Sebbene sino ad oggi io non abbia trovato questa bella specie nella Provincia veronese, pure credo opportuno inserirla in questo luogo; il chiarissimo Trevisan indicandola fra Peschiera (provincia di Verona) e Sirmione (provincia di Brescia), nelle rupi che prospettano il Lago di Garda. Il carissimo amico Gregorio Rigo mi ha favorito un esemplare di $P$. cretica della finitima provincia di Brescia, e raccolto pure, come risulta dalla scheda, nelle rupi che si specchiano nel Benaco. 2

Tribus 2. - Aspleniaceae.

Gen. V. - Blechnum.

22. Blechnum Spicant Roth tent. germ. 1II, p. 44; Pollin. fl. veron. III, p. 292, et herb!; Vis. et Sacc. cat. p. 12; Blech. 
num boreale Siv. syn. fil. p. 115; Bertol. fl.ital. crypt.pars 1, p. 93; Osmunda Spicant L. sp. pl. 1522.

Rarissima. 2 - 11 Pollini non cita questa felce fia le piante veronesi. Carlo Tonini la raccolse nei monti di Tregnago (Bertol. loc. cit. p. 94); - Abramo Massalongo al confine della Provincia veronese con la vicentina presso Crespadoro (herb. Da Campo!).

\section{Gen. VI. - Athyrium.}

23. Athyrium Filix-femina lioth tent. germ. III, p. 65; Bertol. fl. ital. crypt. pars I, p. 80; Vis. et Sacc. cat. p. 14; Polypodium Filix-femina $L . s p \cdot p l .1551$; Aspidium Filixfemina Sw. fil. 59; Pollin. viag. etc. p. 113; fl. veron. III, p. 282, et herb.!; Asplenium Filix-femina Bernhard. in Schrad. neu. Journ. 1, pars II, p. 27, tal. 2, f. 7; Filix non ramosa, petiolis tenuissimis et tenuissime dentatis Segu. pl.veron. III (Suppl.), pag. 53; Felce femina Pona MI. Baldo p. 230. - Vernacolo: Felese!, Felese femina!, e presso $\mathrm{Ba}$ dia Calavena col nome di Fóldare (fratelli conte Francesco e Carlo Cipolla).

$\beta$ multidentatum Doell. Gefüsslirypt. Bad.p. 23; Milde fil. etc. p. 50. - a Lamina tripinnatisecta: segmentis tertii - ordinis undique dentatis .

$\gamma$ fissidens Doell. loc. cit.? - Lamina rigida, lanceo- lata, bipinnatisecta, $27 \mathrm{~cm}$. longa, $95 \mathrm{~mm}$. lata: segmen- tis secundi ordinis pinnatifidis: lacinulis fere omnibus - apice bidentatis. Planta pallide viridis, sori pauci; segmenta - quamplura sterilia. '

Frequentissima: si incontra ad ogni passo nel M. Baldo, nei Lessini e nei monti che ne dipendono; scendendo dalla regione alpina nella zontana, e penetrando benanco, sebbene più di rado, nella collina. Da aprile e maggio ad inoltrato autunuo. $z_{-}$- La var. $\beta$ cresce frequentissima in tutta la catena del Baldo, specialmento in Basiana, Novesa, Ortigara ccc. Riferisco con dubbio alla var. fissidens Docll., Milde, una bella forma, che meriterebbe forse di essere considerała come distinta varietà, da me raccolta sul versante occidentale di M. Baldo in Val grande (2100 mt.).

Il chiarissimo Trevisan segnala nelle alpi vicentine, a destra del passo della Lora, l'Athyrium alpestre Nyland. (Syn. Poly podium alpestre Hoppe; P. rhaeticum $L$.; P. molle All.): è quasi certo che lo stesso debba crescere nella provincia nostra, nei monti che separano questa dalla finitima provincia di Vicenza, come pure nelle parti più settentrionali della catena del M. Baldo. 


\section{Gen. ViI. - Asplenium.}

24. Asplenium viride Huds. $f$ l. angl. ed. I, p. 385, et herb.; Willd. n. 19912; Pollin. viag. p. 113, et fl. veron. III, p. 286, et herb.!; Bertol. fl. it. crypt. pars I, p. 70; Vis. et Sacc. cat. p. 11. - Vernacolo: Felesina!

$\beta$ inciso-crenatum Milde fil. etc. p. 60; Goir. app. bot. in cron. alp. p. 69. - Segmenta profunde inciso-crenata. .

$\gamma$ pusillum Goir. in herb. - Planta pusilla: lamina - 35-60 mm. longa: segmenta distincte petiolata, rhombea, - irregulariter crenata. ,

Frequentissimo nelle rupi dei monti Veronesi dalla regione montana ai gioghi più elevati. Nei Lessini lungo tutta la catena; nei monti che segnano il confine fra la Provincia veronese, il Trentino ed il Vicentino; nel Baldo alla Corona!, in Val Andrine!, Ime!, Valfredda!, Artillon!, nelle ralli alpine prospicienti il Benaco! ecc. fra $776 . \mathrm{m}$. e $2200 \mathrm{~m}$. di altitudine; il prof. A. Massalongo (Da Campo herb.!) lo ha raccolto nel M. Belloca $(830 \mathrm{~m}$.$) ; qualche volta però scende al basso nelle valli e si arvi-$ cina alla pianura; Carlo Tonini lo osservò in Valpantena tra Alcenago e Stalavena a $2 \hat{\mathrm{u}} 0 \mathrm{~m}$. circa sul mare; e nell'Erbario Da Campo! si trovano esemplari raccolti da A. Massalongo presso Tregnago nella valle dei Finetti $(316 \mathrm{~m}$.). Nella var. $\beta$ mi sono imbattuto per la prima volta nel novembre 1877, lango la salita dal Vò, sulla sinistra dell'Adige, al passo della Sega a circa $200 \mathrm{~m}$. sui mare. La var. $\gamma$ in M. Baldo al Telegrafo (2200 m.), all'Altissimo di Nago (2117 m.), e nelle alpi confinanti col Trentino alla Cima di Posta $(2120 \mathrm{~m}$.$) . - Si mantiene in regetazione e$ sporificato durante tutto l'anno! 2

25. Asplenium Trichomanes Huds. flora angl. ed. I, p. 385; Pollin.viag. p. 113, et fl. veron. III, p. 285, et herb.!; Bertol. fl. it. crypt. pars I, p. 67, var. $\alpha$; Franc. Font. cat. p. 28; Vis. et Sacc. cat.p. 11; Politrico Pona M. Baldo p. 142 et 230?; Trichomanes seu Polytricum officinarum Segu. pl. ver. I, p. 71; Moren. herb.!. - Vernacolo: Felesina!, Felesina dei muri!, Sardelette!, Errba da omo!.

$\beta$ alpestre Goir. app. bot. in cron. alp. etc.p. 69; A. Trichomanes $L$. forma alpestris Goir. specim. morphogr. veg. p. 12. - A forma genuina partibus minoribus facillime distinguitur. Lamina, rachide excepta, laete viridis. .

$\gamma$ microphyllum Tin. ap. Guss. fl. sic. syn. II, p. 884; Goir. app. bot. in cron. alp. p. 69. - Planta pusilla: fron- 
- dibus brevibus, decumbentibus, rosulatis, laete virentibus:

- pinnulis profundius crenatis quam in forma genuina. '

$\delta$ rotundatum Milde fil. etc. p. 61. - Planta elata:

- segmentis late rotundatis, crenatis. '

$\varepsilon$ dentatum Goir. et Tonini. - Frondibus flaccidis,

- decumbentibus: segmentis rhomboideis, basi cuneatis,

- apice truncatis, toto margine, basi excepta, eleganter et - profunde dentatis. ,

Pianta frequentissima nella intera Provincia : dalla pianura si innalza a tutta la regione montana e subalpina, e penetra benanco, sebbene più parcamente, nella alpina, vivendo di preferenza nei muri vecchi e nelle spaccature delle rupi. Si conserva in vegetazione e sporificata durante tutto l'anno, almeno nelle stazioni non troppo aride, ed in quelle riparate dai rigori invernali. $\tau_{-}$- La var. $\beta$ si incontra nelle stazioni elevate dei monti, tanto nel Baldo che nei Lessini; la var. $\gamma$ nelle siepi dei colli veronesi, nelle caverne e nelle grotte; la var. $\delta$ qua e là nei colli e nei monti; la var. $\varepsilon$ nel collo di $S$. Leonardo.

Riferisco con dubbio il sinonimo di Pona, il quale a pag. 143 del suo Monte Baldo nomina l'uno e l'altro Politrico, ed a pag. 230 il Politrico aureo maggiore e minore degli erbarij. E assai difficile potero affermare a quale pianta alluda il Pona. Il Bertoloni, che reca sempre il sinonimo del Pona, in questo caso non lo cita, contrariamente a quanto fu fatto dal Pollini. Il Seguier ( $p l$. veron. $I, p$. 71) osserva : Alteram speciem Pona memorat, quae forsan, muscus est, de qua nihil certe in presentia habeo. Il sospetto del Seguier è tanto più fondato, inquantochè la pianta dal Pona registrata a pag. 230 del suo viaggio è segnata all'Artillon: ora mi sembra poco probabile che il Pona, ad indicare la presenza dell'A. Trichomanes, scegliesse di preferenza una stazione alpina; nella quale questa felce si trova assai parcamente, ed in esemplari lontanissimi dall'offerire quello splendore di forma e di vegetazione, che presenta nelle stazioni per altitudine anche di poco inferiori a questa. Del resto colla denominazione di Politrico aureo, gli antichi scrittori e lo stesso Pollini (fl. ver. III, 312) denominano il Polytrichum commune: il quale Musco cresce copiosissimo in tutta la catena del Baldo.

L'egregio amico, cav. G. B. Barla, micologo distintintissimo e direttore del Civico Museo di Storia Naturale in Nizza, esaminando la composizione del così detto Thé suisse, trovò nei pacchi da lui osservati, che la pianta dominante era per lo appunto l'A. Trichomanes.

26. Asplenium fontanum Bern. in Schrad. Journ.; Vis. et Sacc. cat. etc. p. 275.

Sinora non mi venne dato di imbatterni in questa felce. I signori R. de Visiani e P. A. Saccardo, nel loro eccellente Catalogo delle piante 
vascolari del Veneto, la indicano nel M. Baldo sulla affermazione del sig. John Ball (Veggasi Guida alpina, Sezione 57, traduzione del conte Giulio Giusti pag. 38 , sub A. Halleri).

27. Asplenium Ruta-muraria $L . s p . p l$. 1511; Pollin. $f$. veron. I.lI, p. 287, et herb.!; Bertol. fl. it. crypt. pars I, p. 71; Franc. Font. cat. p. 28; Vis. et Sacc. cat. etc. p. 11; Ruta-muraria Segu. pl. veron. I, p. 72; Moren. herb.!. - Vernacolo: Felesina!, Capiler mato!, Capiler bastardo!.

a. Stirps mire polymorpha; inter coeteras, sequentes - prestantiores formas, in ditione nostra lectas, enumerare - liceat : ग

$\beta$ glandulosum. - A. Ruta-muraria var. Brunfelsii Heufler, forma glandulosa Milde fil. etc.p. 76. - "Frondibus - sordide canescentibus!: rachide segmentisque glanduloa sis: glandulis pedicellatis.

$\gamma$ pseudo-germanicum Heufler; Milde Fil. etc. p. 77. Folia angusta, longissime petiolata, 3-pinnatisecta, opaca; segmenta ultima pauca (1-juga), angusta, elongato-cuneata, incisodentata, dentibus linealibus longis. Asplenio germanico non absimile (Milde loc. cit.).

$\delta$ pulchellum Goir. - Frondibus $8 \mathrm{~cm}$. altis, petio- lis cm. 5, 5 longis: lamina ovato-lanceolata, tripinnati- secta: segmentis ultimis minutissimis, ovalibus, $1 \mathrm{~mm}$. la- tis, 1, $5 \mathrm{~mm}$. longis, integerrimis, omnibus fertilibus; soris ' densissimis.

Nei muri vecchi e nelle rupi soleggiate dalla pianura ai monti, ed anche nella regione alpina ove si fa più rara. - La var. $\beta$ in M. Baldo nei pressi della Corona!. La var. $\gamma$, che nel portamento ricorda l'A. germanicum, è assai diffusa: io la ho raccolta nel M. Baldo presso la Corona $(776 \mathrm{~m}$.$) , ad Olivè presso Montorio, alla Mosella (91 \mathrm{~m}$.$) . La var. \delta$ è una vaga piantina che nel settembre del 1874 ho trovato per la prima volta in M. Baldo presso la Corona.

La Ruta-muraria si mantiene in vegetazione e sporificata durante tutto l'anno, almeno nelle stazioni più favorite. 20

28. Asplenium septentrionale $S w$. syn. fil. p. $75 ;$ Pollin. ind. delle piant. racc. sui coll. Eng. p. 24, et fl. veron. III, p. 285; Bcrtol. fl. it. crypt. pars I, p. 85; Vis. et Sacc. cat. etc. p. 12; Acrostichum septentrionale L. sp. 1524; Filicula saxatilis corniculata Segu. pl. veron. I, p. 70; Moren. herb.!. 
Rarissimo. 2 - Nel M. Baldo (Tonini in Bertol. loc. cit.), e presso Revolto nei Lessini (A. Mass. in herb. Da Campo!, esemplare imperfettissimo). Gli esemplari esistenti nell'Erbario Polliniano non appartengono al Veronese.

29. Asplenium fissum Kit. ap. Willd. sp. pl. $\nabla^{\circ}, p .348$; Bertol. fl. it. crypt. pars I, p. 78; Milde fil. etc. p. 83-81; cump. fl. ital. p. 19; Vis. et Sacc. cat. p. 11; A. Breynii Pollin. fl. veron. III, p. 286 (excl. omn. syn.), et hert.!; A tenuifolium Guss.; A. Trettenerianum Jan. - Icon.: Gussone pl. rar. t. 65.

Rarissimo ai confini del Veronese colla Provincia vicentina e col Trentino: io ho raccolto questa felce al Passo della Lora (1726 m.), lungo la discesa verso Recoaro, ove è pure indicata da Pollini e dallo Jan: si trova benanco nel M. Campobruno, e presso Revolto a sinistra del Passo ora detto, guardando verso nord; ed il prof. A. Massalongo la rinvenne presso Giazza (herb. Da Campo!). - Si può raccogliere sporificata alla fine dell'estate e nell'auturno. 2

L'A. Breynii del Pollini corrisponde sicuramente allo A. fissum Kit.; i sinonimi però e le figure citate da lui sono da riferirsi al vero $A$. Breynii Retz. (1774), che per posteriorità di data diventa sinonimo dell'A. germanicum Weiss (1770). A torto poi il Pollini sospetta che la pianta in quistione possa essere una semplice varietà dell' $A$. Ruta-muraria, ex loci asperitate orta. E l'errore del Pollini deriva da ciò che, come sembrerebbe risultare dall'esame degli esemplari che sono nel suo erbario, il chiarissimo Autore della Flora Veronese ha confuso con il vero $A$. fissum taluna forma spettante all'A. Ruta-muraria. Gli esemplari Veronesi di A. fissum concordano pienamente con gli Austriaci del Bolle ed altri, e con gli Abruzzesi raccolti dal dottor Levier.

Sino ad oggi l'A. germanicum Weiss non è stato rinvenuto, per quanto io so, nei monti veronesi : non credo però sia da escludersi affatto la probabilità o possibilità della sua presenza nelle nostre alpi, questa felce crescendo nelle finitime provincie del Vicentino e del Bresciano. Così pure non è improbabile che nelle nostre dolomie possa ritrovarsi l'Asplenium Seelosii Leybold, da chiarissimi botanici (Milde fil. etc. pag. 76) segnalato nel vicino Trentino, e presso il lago di Garda nella provincia di Brescia.

Noto infine che per pura svista, il Bertoloni ( $f$. it. crypt. pars I, p. 73) cita fra i sinonimi dell'A. germanicum, l'Asplenium Breynii della Flora Polliniana.

30. Asplenium Adiantum-nigrum L. sp. pl. $1542 ;$ Pollin. fl. veron. III, p. 288, var. a et $\gamma$; Bertol. fl. it. crypt. pars 11 , p. 74; Vis. et Sacc. cat. p. 12, var. $\alpha$ et $\beta$; Filicula quae Adianthum nigrum officinarum, pinnulis obtusioribus 
Segu. pl. veron. I, p. 69; IIoren. herb.! - Vernacolo: Polipodio cavallin. - Icon.: Pollin. fl. veron. III, t. II, f. 2, sub Aspidio acuto.

- Formae veronenses a me lectae, omnes ad A. ni- grum referendae videntur. Duae praesertim varietates vel - potius variationes, mihi obviam fuere: •

a lancifolium Heufler; Milde fil. etc. p. 85. - . Lamina - anguste lanceolata: segmentis ultimis ovatis, acute dena tatis. Frondes hyeme perdurantes, ut in sequenti. "

$\beta$ obtusum Kit.; Milde fil. etc. p. 85. - - Lamina late - ovata: segmentis ultimis e basi angustiore, obtusis, den- tatis: dentibus plerumque acutis. .

- Prope Roma, extra Porta Portese in quodam dumeto - (sept. 1874), legi formam monstrosam, lamina apice bi- fida.

Nei colli e nei monti della Provincia durante tutto l'anno; e non sembra escire, almeno in via generale, dai limiti della regione montana: in Val Policella presso Fumane!; nei colli veronesi nel Vajo del Borago, presso Avesa!, nelle vicinanze di Montecchio nei basalti!; nella Valle Pantena presso Alcenago! (e Tonini!); nella Valle di Illasi nel Vajo dei Rugolotti!; nel M. Belloca (her.. Da Campo!); nel M. Bolca!; nel Vajo di Squaranto! ecc. - La var. $\beta$ fra i kasalti nei colli di Lavagno!. Il Seguier colla frase pinnulis obtusioribus caratterizza molto bene le forme veronesi spettanti a questo tipo.

L'Asplenium acutum di Ciro Pollini Hort. et prov. veron. pl. nov. vel min. cogn. (1816) pag. 28, et fl. veron. III, pag. 288, mi sembra diverso dall'A. Virgilii Guss., stando alla figura del chiarissimo Autore, da me confrontata con esemplari genuini di quest'ultimo provenienti dall'Italia meridionale. E col Bertoloni opino, che la pianta del Pollini sia una semplice variazione del vero $A$. Adiantum-nigrum $L$.; la quale non esiterei punto a riforire alla var. nigrum Heufler.

\section{Gen. VIII. - Scolopendrium.}

31. Scolopendrium vulgare Symons syn. p. 193; Vis. et Sacc. cat. p. 12; Milde fil. etc. p. 88.90; Scolopendrium officinarum Sw. syn. fil. p. 89; Pollin. viag. etc. p. 29, et fl. veron. III, p. 289; et herb.!; Bertol. fl. it. crypt. pars I, p. 82; Fillitide di Dioscoride Pona IM. Baldo p. 142; Lingua cervina officinarum Segu. pl. veron. I, p. "rt; Moren herb.!. - Vernacolo: Lengua zzervina, Regolizia. 
- Stirps polymorpha: facillime in formas monstrosas " transit. In ditione nostra sequentes occurrunt formae: , a genuinum.

$\beta$. Planta pumila. Lamina, in speciminibus nostris, "4-10 cm. longa, 9-25 mm. lata. - S. minus Fée gen. fil. p. 209. - Formam monstrosam legi ad hanc varietatem per- tinentem, lamina basi rotundato-cuneata, in uno latere ap" pendice rotunda protracta. o

$\gamma$. Lamina basim versus contracta, apice bifida: la- ciniis brevibus, rotundalis, divaricatis. , - - . vulgare $\beta$ furcatum Goir. spec. morphogr. veg. p. 12; S. officinarum var. bifidum F'é gen. fil. p. 209.

$\delta$ a Lamina $40 \mathrm{~cm}$. longa, 9-10 cin. lata, ad basim - contracta, apice subito acuminata: ba:eos lobis patentis" simis. - - S. vulgare $\gamma$ platyphyllum Goir et Ton.; Goir. spec. morphog. veg. p. 12.

$\varepsilon$ - Lamina undulato-crispa, saepe margine et apice - inciso-dentata (in speciminibus veronensibus), vel inciso- lobata. Forma monstrosa summopere varians quae tran- sit in formam daedaleam. - S. officinar im var. crispa Koch " syn. fl. germb. et helv. ed. 2, p. 984; Milie fil. etc. p. 89.

Volgarissimo nella Provincia, si mantiene in vegetazione durante tutto l'anno; e dalla bassa pianura ascende alla regione montana e penetra benanco nella subalpina. 20 - La var. $\beta$ nei luoghi umidi delle convalli presso il Maso! e Montecchio!, e nella Valle Paittena presso Alcenago! e Stalavena!, specialmente nelle rupi e nelle tufe basaltiche. La var. $\gamma$ nel vaio dei Rugolotti!, e nei dintorni di Verona presso Avesa!. La var. $\delta$ assieme alla forma normale presso Avesa!. La var. $\varepsilon$, forma mustruosa od anomala che Ciro Pollini (loc. cit.) dice di non avere mai incontrata nella Provincia, si osserva abbondantissiza nelle pittoresche convalli dei monti vicini ad Aresa. Non mi è mai cavitato però d' incontrare individui, nei quali tutte le frondi presentassero i caratteri propri di questa anamorfosi: sempre sopra lo stesso rizoma ho trovato frondi modificate in unione ad altre interissime e piane nei margini ed all'apice. Il chiarissimo Bertoloni (fl. it. erypt. pars I, pag. 84) scrive: frondem apice laciniatam possideo ex alienis locis non ex Italia; nel settembre 1878, io ho raccolto questa forma nei dintorni di Roma. Questa forma si trova pure nell' Erbario legnaghese del Rocchetti assieme alla var. crispa, ma sospetto provenga da qualche giardino.

Alcune forme di S. vulgare, segnatamente la crispa e la daedalea, sono coltivate nei giardini veronesi, e figurano nelle sale delle nostre вignore. 


\section{Gen. IX. - Ceterach.}

32. Ceterach officinarum $W . s p . p l . V, p .136 ;$ Pollin. $f$. ver. III, p. 272, et herb.!; Bertol. fl. it. erypt. pars I, p. 38; Vis. et Sacc. cat. p. 9; Asplenium Ceterach L. sp. pl. 1538; Asplenio di Dioscoride e Ceterach degli Arabi Pona M. Baldo p. 142; Asplenium sive Ceterach Segu. pl. veron. I, p. 74; MLoren. herb.!. - Vernacolo: Felese dei muri!, Capiler bastardo!, Erba dorada!.

Nei muri vecchi e nelle rupi copiosissima: dalla pianura, Legnago! (ex herb. Rocchetti!), Villa Bartolomea!, Verona!, sponde del Benaco! ecc. si innalza alla regione montana e penetra benanco nella subalpina, p.e. nel M. Baldo sin sopra la Ferrara!. - - Si mantiene in vegetazione e sporificata durante tutto l'anno. 20

\section{Tribus 4. - Aspidiaceae.}

Gen. X. - Phegopteris.

33. Phegopteris Dryopteris Fée gen. fil. p. 243; Milde fil. etc. p. 98; Polypodium Dryopteris L. sp. pl. 1555; Pollin. fl. veron. III, p. $2 r^{\prime} 4$ pro parte, et herb.!; Bertol. fl. it. crypt. pars 1, p. 46; Vis. et Sacc. cat. p. 9; Dryopteris Calc. viag. p. 8; Driopteri Pona II. Baldo p. 144; Filix ramosa minor, pinnulis dentatis $S e g u$. pl. veron. I, p.68. Vernacolo: Felese.

Si incontra qua e là nei monti veronesi dalla regione montana alla alpina: nei $M$. Lessini presso Cerro! e Revolio (C. Massalongo!), nel $M$. Baldo, nel $M$. Zeola, nel $M$. Bolca. Le frondi spuntano da aprile a giugno secondo l'altitudine; sono sporificate in estate ed autunno, e persistono in via generale sino a novembre e dicembre. 2

34. Phegopteris Robertiana A. Br.; Milde fil. etc. p. 99; Ph. calcarea Fée gen. fil. p. 243; Polypodium calcareum Smith; P. Dryopteris var. $\beta$ Bertol. fl. it. crypt. pars I, p. 47; P. Dryopteris $\beta$ calcareum, Vis. et Sacc. cat. p. 9; Goir. pl. vasc. etc. p. 18. - Vernacolo: Felese.

Cresce copiosissima mescolata alla precedente, della quale è ritenuta da molti botanici come una semplice varietà : e mie osservazioni particolari mi hanno convinto che questa seconda è di gran lunga più frequente della 
prima, specialmente nei crepacci delle rupi calcarec. Si avanza pure nella regione dei colli, sino quasi al limite della pianura : cos̀ ad es. si trova nelle fessure delle rupi in Val Pantena sopra Quinto e Pojano!. Il Tonini segnalo per il primo questa specie, indicandola nei pressi di Selva di Progno!. Da aprile e maggio ad ino'trato autunno: nella regione dei colli, io la ho osservata in vegetazione anche nel cuore dell'inverno. 2

Il Polypodium Dryopteris Pollini, e così pure le piante di Calceolari, Pona e Seguier citate nella sinonimia al precedente, abbracciano entrambe queste due specie: il Driopteri indicato dal Pona nella Valle di Caprino spetta secondo ogni probabilitì alla seconda. Noto che la presenza di ghiandole, secondo il mio modo di vedere, non è carattere esclusivo della Ph. Robertiana; avendo osservato in molti punti della catena delle Alpi, individui appartenenti sicuramente alla vera $P$. Dryopteris, i quali e sulla rachide e nei lobi dei segmenti delle frondi portavano ghiandole, sebbene in poca quantità: potrebbe darsi si trattasse di una forma ibrida.

35. Phegopteris polypodioides Fée gen. fil. $p$. 243; Polypodium Phegopteris L. sp. pl. 1550; Pollin. viag. p. 113, et fl. ver. p. 273, et herb.!; Bertol. fl. it. crypt. pars I, p. 15; Vis. et Sacc. cat. p. 9. - Vemacolo: Felese.

Nei luoghi selvatici della regione alpina e subalpina, scendendo benanco nella parte superiore della montaua: nel M. Baldo a Campion!, Acque negre!, Lavacci!, Pian della Cenere!, Artillon!, Altissimo di Nago!, nelle ralli alpine prospicienti il Benaco! ecc.; nei Lessini ai Trachi!, a Revolto ecc.; nel MI. Zeola! (e Caro Massalongo). - Compaiono le frondi al liquefarsi delle nevi; sono sporificate da metà estate ad inoltrato autunno. 2

\section{Gen. XI. - Aspidium.}

36. Aspidium Lonchitis Sw. in Schrad. Journ. (1800) II, p. 30 ; syn. fil. p. 43; Pollin. viag. p. 113, et fl. veron. III, p. 2\%5, et herb.!; Bertol. fl. it. crypt. pars 1, p. 49; Polypodium Lonchitis L. sp. pl. 1548; Hypopeltis Lonchitis Vis. et Sacc. cat. p. 10; Lonchite aspera Calc. viag. p. 11; Lonchite aspera maggiore del Ghini Pona $M$. Baldo p. 171; Lonchite aspera maggiore Pona op. cit. p. 244; Lonchitis aspera Segu. pl. ver. I, p. 71; Moren. herb.!.

Nella regione subalpina ed alpina dei monti veronesi; più raramente nella montana. Nel M. Baldo in Basiana!, fra le rupi di Valfredda (Pona), Val Losana! (e Pona), Campion!, Lavacci (Pollini), Pian della Cenere! (e Pollini), Novesa (Pollini), Artillon! (e Pollini, e Toninil), in tutte le 
valli alpine che prospettano il Benaco!; cresce pure nei Lessini!, nel $M$. Zeola!, nel Mr. Bolca!, nei Monti di Tregnago! etc. - Estate-autunno: qualche volta le frondi persistono durante il verno. $z_{0}$

37. Aspidium aculeatum Döll. vhein. Flora (1843) p. 20; Bertol. fl. ital. cript. pars I, p. 54.

- Stirps polymorpha: attamen formae variae ad Aspi- dium aculeatum pertinentes, facile typis duobus sequenti" bus referendae videntur. ,

a lobatum Sw. syn. fil. p. 53; Aspidium aculeatum Pollin. fl. veron. III, p. 279, var. $\beta$; Hypopeltis aculeata $\beta$ lobata Vis. et Sacc. cat. p. 10.

$\beta$ aculeatum Sw. loc. cit.; Aspidium aculeatum Poillin. viag. var. $\alpha$ et $\beta$ p. 113, et fl. veron. p. 27s, var. $x ;$ Hyporeltis aculeata var. a Vis. et Sacc. cat. p. 10. - Vernacolo: Felese.

Le due forme crescono promiscnamente dai colli ai monti fra $150 \mathrm{~m}$. e $2000 \mathrm{~m}$. di altitudine: nei colli di Valpantena al disopra di Quinto e Grezzana!; nel M. Baldo a Campion!, Acque negre! (e Pollin.), Pian della cenere! (e Pollini), Fassole!, Lavacci! (e Pollini), nelle valli alpine che so!cano il versante occidentale del monte! erc.; nei M. Lessini in tutta la catena!; in val d' Illasi ai Rugolotti!; nel M. Bolca! (e Caro Massalongo!). - Estate ed autunno nelle stazioni elevate; durante tutto l'anno nei colli. $z$

Nel colle delle Fassole in M. Baldo, ho raccolto fra i Rododendri, una forma sterile nella quale i segmenti della lamina sono pennatifidi nella parte mediana di questa: la profondità delle lacinie però va gradatamente diminuendo passando da un segmento all'altro, talchè i superiori $\epsilon d$ anche gli infimi sono finamente dentati. In questa forma anomala poi, il lobo infimo che sta nella parte superiore dei segmenti, da prima nettamente distinto e separato, si accosta man mano a quello che sta inmediatamente appresso, si congiunge con esso, e nei segmenti supremi figura come una orecchietta collocata alla base superiore degli stessi.

La var. $\gamma$ dell' $A$. aculeatum della flora di Ciro Pollini (l. c. p. 279) e raccolta dallo Zantedeschi presso Bovegno nei monti Bresciani, è una semplice forma.

38. Aspidium montanum Vogler dissert. de Polypodio montano, Gissae 1781; Milde fil.etc. p. 115; Aspidium Oreopteris Sw. syn. fil. p. 50; Pollin. fl. veron. III, p. 276; Bertol. fl. ital. crypt. pars I, p. 61; Polystichum Oreopteris Vis. et Sacc. cat.p. 10; Nephrodium Oreopteris Roeper Fl. Meclilbrg.p. 81.

Raro: nel M. Baldo (Sternberg); nel M. Zeola; nel M. Bolca (Pollini, Caro Massalongo!). - Estate-autunno. 2 
39. Aspidium Thelypteris Sw. syn. fil. p. 50; Pollin. viag. etc. p. 14, et fl. veron III, p. 277; Bertol. fl. it. crypt. pars I, p. 52; Polystichum Thelypteris Vis. et Sacc.cat. etc. p. 10; Filix minor non rainosa Segu. pl. ver. 1, p. 67. Vernacolo: Felese.

$\beta$ Rogaetzianum Bolle; Milde fil. etc. p. 117. - . La- ciniae fertiles dilatatae, margine planae. ,

Nelle risaie, nei fossi e nei luoghi palustri della pianura frequentissimo: presso S. Michele!, Legnago! (e Rocchetti herb.!), Oppeano (Seguier, Pollini et herb.. Da Campo!), Palù (Seguier) Villa Bartolomea! (e Pollini), Peschiera! (e Pollini) ecc. - Da giugno ad ottobre. $z^{\circ}-$ La var. $\beta$ è più frequente della forma genuina.

40. Aspidiun Filix-mas $S w$. in Schrad. Journ. p. 39, et syn. fil. p. 55 ; Pollin. viag. p. 80, et fl. veron. III, p. 279; Bertol. fl. it. crypt. pars I, p. 56; Polystichum Filix-mas Vis. et Sacc. cat. p. 19; Nephrodium Filix-mas Strempel fil. berol. p. 30; Felce maschio Calc. viag. p. 10; Pona Monte Baldo p. 15, 15\%, 230; Filix non ramosa dentata Segu. veron. I, p. 67; Moren. herb!. - Vernacolo:Felese, Felese mascio. Gli egregi amici, Conti Francesco e Carlo fratelli Cipolla, nelle loro accuratissime ricerche sul dialetto Cimbro dei Sette Comuni, hanno trovato che alla Giazza (Veronese), l'Aspidium Filix-mas, l'Athyrium Filixfemina e la Pteris aquilina ricerono la denominazione comune di Fliöute, e al plurale Fliöuter. Queste stesse piante, più a basso nella Valle d'Illasi, a Badia Calavena, come già ho accennato, vengono indicate col nome di Fóldare.

a genuinum Milde fil. etc. p. 119. - " Laciniae mar- gine subintegerrimae, apice rotundato densius serratae. •

$\beta$ crenatum Milde loc. cit.; A. Filix-mas Bertol. fl. ital. crypt. pars I, p. 57 var. $\beta$; Polystichum Filix-mas $\beta$ erosum Vis. et Sacc. cat. p. 10; P. Filix mas $\beta$ crenatum Trevisan syll. sporophyt. It. var. $\beta$ in atti della Società Italiana XVII, p. 226; Aspidium cristatum Pollin. fl. veron. III, p. 278! non $S w$. et excl. syn. - "Laciniae margine et apice rotundato! - vel subacuto!, manifeste serratae et plerumque grosse den. - tatae. Ceterum var. $\$$ transit ad formam genuinam. Vidi 
- quoque in Alpibus specimina, laciniis margine et apice - integerrimis vel subintegerrimis. "

Dalla pianura e dai dintorni di Verona alla regione alpina. Nel Bosco Mantico!, nelle vallette dei colli veronesi !, nel Baldo!, nel Pastello!, nei Lessini! e nei monti che ne dipendono copiosissino. La varietà $\beta$ nel M. Bolca (Pollini!, Caro Massalongo!) ; nel M. Baldo in Valle Basiana!, alle Acque negre!, Campion! etc. La forma raccolta in Basiana di M. Baldo, ha un portamento che ricorda quello dell'Aspidium rigidum col quale, a dire il vero, a prima vista io la arevo confusa : merita di essore vilteriormente studiata e forse di venire incalzata al grado di distinta varietà. - Estate ed autunno: in alcune stazioni più riparate, si mantiene anche nell' in erno. 2

L'Aspidium cristatum del Pollini spetta sicuramente alla nostra var. $\beta$, come testifica il Bertoloni sugli esemplari ricevuti dal chiariss. Autore della Flora veronese; e come risulta benanco da quelli che si conservano nell'Erbario Polliniano, sebbene sieno andati mescolati assieme ad altri di dirersa forma. - - Il Pollini riferisce con dubbio al suo A. cristatum la Filix non ramosa alpina, alis rarioribus, et pinnulis sinuatis, foliis autem dentatis di Pon'edera (comp. tab. bot. 13) e Seguier (pl. veron. I, 67). Nella mancanza di esemplari autentici, credo sia assolutamente impossibile ogni congettura sulla pianta voluta veramente indicare da questi autori.

Nelle nostre montagne l'Aspidium Filix-mas e l'Athyrium Filixfemina, sono ovunque 'estinati a quell' istesso uso che superiormente è stato indicato per la Peris aquilina.

41. Aspidium rigidam Sw. in Schrad. Journ. p. 3\%, et syn. fil. p. 53; Pullin. viag. p. 114, 117; fl. veron. III, p. 281, et herb.!; Brrtol. fl. ital. crypt. pars I, p. 61; Polystichum rigidum $V$ is. et Sacc. cat. p. 10; Nephrodium rigidum Desv. annal. Lim. VI, p. 261; Filix non ramosa alpina foliolis quae ad alas rotundioribus, omnibus autem dentatis Ponted. comp. tab. bot. p. 13, n. 13; Segu. pl. veron. III (suppl.), p. 53; Moren. herb.!. - Vernacolo: Felese. Icon. Segu. loc. cit. t. I, f. 1.

$\beta$ pusillum Goir. in herb. - a Planta pusilla: lamina 5-7 - cm. longa; $2-3 \mathrm{~cm}$. lata. "

Cresce copiosamente nei luoghi pietrosi alpini ed anche subalpini del M. Baldo,tanto nel versante occidentale che nell'orientale: a Costabella!, Val vaccara!, Valle delle buse!, Valle delle pietre!, Val grande,! Valle degli ossi!, Val finestra!, Val Losana!, Acque negre!, Altissimo di Nago! ecc.; nei Lessini nei gioghi più elevati!; nel M. Campobruno ed in Cima di Posta!; nel M. Zeola!; nei monti dlba!, Gramellon! ecc. - Ia var. $\beta$ in 
Val grande di M. Baldo ad nivem deliquescentem. Forse taluna delle forme raccolte uelle valli alpine di M. Baldo potrebbe riforirsi alla var. IIypodematium Trevis. syll. sporophyt. It. in Atti della Società Italiana di Scienze Naturali XVII, p. 230. - L。 frondi cominciano a svilupparsi allo squagliare delle nevi; sovo sporificato in estate ed autunno. 2

42. Aspidium eristatum Sw. in Schrad. Journ. p). 37 (non Pollin.); Goir. app. bot. in cron. alp. p. 69; Pölystichum cristatum Roth tent. fl. germ. III, I, p. 81 .

Raro. Nel M. Baldo nei luoghi umidicci presso Campion! ed alle Acque negre! in mezzo ai frutici, e nella Vall: delle pietre!. - Antunno. 2

A dire il vero gli esemplari raccolti sino ad oggi da me sono tutti sterili : ad ogni modo le frondi vella loro configurazione e struttura concordano esattamente con gli esemplari francesi raccolti dal sig. Magnier presso S.t Quentin nel dipartimento dell'Aisne e comunicatimi dal sig. G. Rouy.

43. Aspidium spinulosuma Sw. s'jn. fil. p. 51; Milde fil. etc. p. 13.; Bertol. fl. ital. crypt. pars I, p. 59.

a genuinum Iiöper zur Filora von Meclilbrg. p. 93; Milde ioc. cit. p. 132; Aspidium dilatatum Pollin. fl. veron. III, p. 280 (excl. syn.?); Polystichum spinulosum Vis. et Sacc. cat. etc. 1). 10. - Segmentis secundariis pinnatipartitis, ala rachidis - angustissima inter se conjunctis: infimis plerumque ste- rilibus. ,

$\beta$ dilatatum Röper loc. cit.; Milde loc. cit.; Aspidium spinulosum Pollin. fl. veron. 1II, p. 281 in nota; Polystichum spinulosum $\beta$ dilatatum Vis. et Sacc. cat. etc. p. 275. a Segmentis secundariis ala rachidis, late expansa, con- fluentibus.

Non ho raccolto ne!le alpi veronesi se non la var. $\propto$; alla quale è da riferirsi sicuramente l'Aspidium dilatatum del Pollini, come risulta dalla frase pinnulis oblongis distinctis... indusiis laevibus (fl. ver. III, p. 280): non è però improbabile cresca benanco la var. $\beta$. La var. $\alpha$ si incontra copiosissima in M. Baldo nella valle alpina delle pietre!, sul versante occidentale, fra i Mughi, i Rododendri, i Vaccini, l'Alnus viridis etc. : così pure nel $M$. Zeola, ove primieramente è stata raccolta da Pollini !, A. Massalongo!, Da Campo!. In M. Baldo ho pure raccolto una forma interamente sterile di A. spinulosum var. $\alpha$, che si distingue dalla fertile per il maggiore sviluppo di tutti i segmenti primari e secondari delia fronda, e per la tinta pallido-flavescente di questa. - Si sviluppano le frondi al fondersi dello nevi: si trovano sporificate in autunno. $z$ 


\section{Gen. XII. - Cystopteris.}

44. Cystopteris fragilis Bernhard. in Schrad. neu. Journ. Bot. I, p. II, p. 27; Vis. ct Sacc. cat. etc. p. 10, excl. var. o; Aspidium fragile Pollin. viag. etc. p. 91, 117, et flor. veron. 1II, p. 282, excl. var. $\delta$; Cyathea fragilis Bertol. fl. ital. crypt. pars I, p. 102-103, excl. var. $\chi$. - Vernacolo: Felese, Felesina.

- Stirps mire polymorpha et ludibunda, ab auctoribus - in species quamplurimas vexata, quae certe formis inter- mediis confluunt. In montibus nostris, sequentes formae a insigniores enumerandae videntur : "

a dentata Dickson; Milde fil. etc. p. 148; Cystopteris fragilis Vis. et Sacc. cat. etc. p. 10, var. $\gamma$; Polypodium Pontederae All. ped. II, p. 286; Aspidium fragile Pollin. viag. p. 103 et 11\%, var. $\beta$, et fl. veron. III, p. 283, var. $\beta$; Cyathea fragilis Bertol. fl. ital. crypt. pars I, p. 101, var. $\delta$; Filicula alpina, foliolis rotundioribus et crenatis Ponted. comp. tab. bot. p. 19; Segu. pl. veron. III, p. 51. - Icon.: Segu. loc. cit. t. I, fig. 2.

$\beta$ pumila Bertol.; Cystopteris fragilis Vis. et Sacc. cat. etc. p. 11, var. A; Polypodium asplenioides Scop. carn. ed. 2, II, p. 298; Aspiaium fragile Pollin. fl. veron. III, p. 283, var. $\gamma$; Cyathea fragilis Bertol. fl. ital. crypt. pars I, p. 104, var. $\gamma$; Filicula alpina latifolia et minor, foliolis dentatis Ponted. comp. taï. bot. p. 19. - Icon.: Scop. loc. cit. t. 62.

$\gamma$ anthriscifolia Milde fiv. etc. p. 148; Cystopteris fragilis Vis. et Sacc. cat. etc. p. 10; Aspidium fragile Pollin. fl. veron. III, p. 282-283, var. a pro parte et excl. syn. Polypodium rhaeticum All.; Cyathea anthriscifolia Roth tent. III, p. 91; Cyathea fragilis Bertol. fl. ital. crypt. pars I, p. 102, var. a pro parte.

o cynapifolia Milde fil. etc. p. 148; Cystopteris fragilis Vis. et Sacc. cat. etc. p. 11, var. $\varepsilon$; Aspidium fragile Pollin. fl. veron. III, p. 282-283, var. a pro parte; Cyathea cynapifolia Roth tent. III, p. 94; Cyathea"fragilis 2 Bertol. fl. ital. crypt. pars $I$, p. 102, pro parte.

$\varepsilon$ angustata IIilde fil. etc. p. 149; Cystopteris fragilis Vis. et Sacc. cat. p. 10, var. $\beta$; Cystea angustata Sm. eng. 
fl. IV, p. 301; Cyathea fragilis Bertol. fl. it. crypt. pars I, p. 103 , var. $\beta$. $\zeta$ stenoloba Al. Br.; Milde fil. etc. p. 149.

Dalle vette alpine questa elegantissima pianta scende alla regione collina, ed internandosi nelle valli si avanza verso il piano. Assume forme diversissime dipendentemente dalla natura e dalle condizioni della stazione nella quale vegeta. Ama in generale i luoghi ombrosi : nei vecchi tronchi degli alberi, nelle siepi, nelle fessure delle rupi umidicce, nelle ghiaie alpine trova condizioni eccellentissime. Conincia a vegetare in aprile nei luoghi più caldi, e si mantiene sino in ottobre ed anche in novembre. 3

La var. $\alpha$ cresce in tutte le valli alpine del Baldo; nelle quali è segnalata dal Seguier, dal Pollini e dal Brignoli che la raccolse in Val Iosana: scende-benanco in istazioni più basse, ed il Tonini ne ebbe un esemplare raccolto da A. Massalongo nel M. Belloca (830 m.). Di questa varietà scrive il Pollini, tanto nella Flora che nel Viaggio, che trasportata dalle valli alpine del Baldo nell' Orto Botanico di Verona, dopo due o tre anni di vegetazione, assunse l'aspetto ed i caratteri della forma più comune del suo Aspidium fragile. - La var. $\beta$ vegeta nelle fessure delle rupi alpine tanto nel M. Baldo che nei Lessini; io la ho raccolta nella Valle delle buse!: ad essa mi sembra debba riferirsi l'esemplare trovato da A. Massalongo alla Giazza ed esistente nell'Erbario Da Campo sotto la denominazione di Aspidium alpinum. Tutti gli esemplari raccolti da me sono completamente sterili: la qual cosa, osservò già il Pollini, riscontrarsi frequentemente tanto in questa che nella varietà precedente. La var. $\gamma$ è la più comune e diffusa, e si incontra ad ogni passo nei nostri monti, dai quali scende ai colli e nelle valli. - La var. $\delta$ è stata raccolta sul M. Baldo dallo Sternberg: di questa si conservano pure esemplari nell' Erbario Padovano, proveuienti dallo stesso monte. - La var. $\varepsilon$ si incontra qua e là, e lo Sternberg ora nominato la segnalò sul M. Baldo. La var. $\zeta$ è rarissima: io la ho trovata in una sola stazione, sul M. Baldo a Costabella! (2060 m.). - Ammetto come un dogma inconcusso la lenta ma incessante metamorfosi, se non di tutte, certo di moltissine forme vegetali: pertanto in questa somma di varietà, in questi lusus come direbbe taluno, vedo l' indefesso lavorio della natura, che nelle specie più polimorfe e per conseguenza atte maggiormente alla lotta per la esistenza, getta lo stampo delle specie avvenire.

Le piante descritte da Seguier con le frasi Filicula fontana major sive Adiantum album Filicis folio, Filicula quae Adiantum album tenuifolium Joannis Bauhini latifolia ( $p l$. veron. $I, p .69$ ), e raccolte da Pontedera nol M. Baldo; quelle indicate dal Martini con le denominazioni di Filix saxatilis ramosa nigris maculis punctata, Filix non ramosa nigris maculis punctata (cat. pl. M. Baldi) e raccolte da lui nelle rupi ai Lavacci, nello stesso M. Baldo, appartengono alla Cystopteris fragilis: ma credo difficile lo asserire a quale forma debbano più specialmente essere riferite. Mi pare 
perd che il Martini nella Filix non ramosa nigris nuaculis punctata indichi una forma che, secondo ogni probabilità, potrebbe corrispondere alla var. dentata.

45. Cystopteris alpina Desv. in annal. Soc. Lin. p. 264.

a genuina Trevis. syll. sporophyt. It. in atti della soc. ital. di scienze naturali XVII, p. 223; C. alpina Linti h. berol. p. 130; Milde fil. etc. p. 150; Vis. et Sacc. cat. p. 11; Aspidium alpinum Pollin. viag. etc. p. 103; A. fragile Pollin. $\mathrm{fl}$. veron. III, p. 284, var. $\delta$; Cyathea alpina Bertol. fl. ital. crypt. pars I, p. 106; Felce crespo sassatile Pona viag. di $M$. Baldo p. 224, e Moren. herb.; Filicula alpina, exilior, pinnulis in tenuissima foliola dentata divisis Ponted. comp. tab. bot. p. 20; Filicula alpina crispa Segu. pl. veron. III, p. 55. - Icon. Pona loc. cit.; Segu. loc. cit. t. I, fig. 3 (optima).

$\beta$ regia Milde fil. etc. p. 151; Trevis. loc. cit.; C. fragilis $\delta$ regia $\nabla$ is. et Sacc. cat. etc. p. 11; Cyathea fragilis Bertol. $f$. ital. crypt. pars. I, p. 103 var. $\chi$.

Questa elegante piantina cresce copiosamente nel M. Baldo ove è stata segnalata dal Pona, Seguier, Moreni, Pollini, Tonini ecc. Io la ho raccolta fra le ghiaie dei torrenti alpini, ed al piede delle rupi umidicce nelle valli degli ossi, grande, Valfinestra, delle pietre, Orsera, Losana ecc. E sporificata da agosto ad ottobre. La var. $\beta$ è più rara: io la ho raccolta al Passo della Lora, al confine della Propincia Veronese con la Vicentina; ad essa potrebbero forse essere riferite benanco alcune forme che ho raccolto nel M. Baldo, le quali crescevano promiscuamente alla forma tipica. 20

Nella Val Losana di M. Baldo, ed in altri punti, mi sono frequentemente imbattuto in esemplari totalmente sterili appartenenti alla vera C. alpina: essi, come ho già indicato per altre forme, presentano nei lobi dei segmenti delle frondi uno sviluppo superiore di molto a quello cho ei osserva negli individui fertili: e la stessa diversità di vegetazione appare benauco in quei campioni che sullo stesso piede portano frondi sterili ed altre più o meno ricche e fornite di sori. Devo pure segnalare alcune forme raccolte nello stesso M. Baldo, che sembrano quasi doversi ritenere quali forme intermediarie fra la $C$. fragilis e la $C_{\text {. alpina: la }}$ qual cosa darebbe ragione a quei botanici che hanno considerato la seconda come semplice varietà della prima.

La figura del Seguier $p l$ veron. I, t. I, fig. 1, non pù̀ convenire alla nostra pianta ; come riconosce lo stesso Seguier (pl.veron. III, p. 55). Il Seguier poi nota che la figura del Morison (hist. III, p. 581, t.4, fig. 27) è copiata, imperfettamente, su quella del Pona. 
Sinora nella nostra regione non è stata, cho io mi sappia, trovata la C. montana Bernh.: però non ritengo impossibile che la stessa possa rinvenirsi o nel M. Baldo, ovvero sulla sinistra dell'Adige nei monti che separano il Veronese dal Vicentino e dal Trentino.

\section{Subordo II. - Osmundaceae.}

\section{Gen. XIII. - Osmunda.}

46. Osmunda regalis L. sp. pl. 1521; Poilin. fl. veron. III, p. $2 \% 0 ;$ Vis. et Sacc. cat. p. 9 ; Osmunda vulgaris et palustris Segu. pl. veron. I, p. 75; Moren. herb.

Rarissima nella Provincia. Secondo il Seguier cresce nei Monti Lessini a breve distanza dal Tinasso, ove io non la ho potuto rinvenire. Il prof. A. Massalongo la raccolse nel. M. Zeola (herb. Da Campo!), ed in vicinanza della Giazza nel luogo detto Minerthall. 2 - Nell'Erbario del Rocchetti si trova un esemplare di Osmunda regalis: ma la scheda non portando indicazione di località, ignoro se sia stato raccolto nella Provincia.

\section{Subordo III. - OpHioglosseae.}

\section{Gen. XIV. - Ophioglossun.}

47. Ophioglossum vulgatum $L . s p . \quad p l$. 1518; Pollin. viag. etc. p. 121, et fl. veron. 111, p. 268; Bertol. fl. ital. crypt. pars I, p. 28; Vis. et Sacc. cat. p. 9; Ophioglosson Calc. viag. p. 10; Ofioglosso del Fucsio o Ennophillo Pona M. Baldo p. 143; Ophioglossum vulgatum Segu. pl. veron. I, p. 75; Moren. herb.! - Vernacolo: Lengua de serpente.

Dai dintorni di Verona (Seguier) alla regione subalpina, ed anche nella alpina, nei prati e pascoli umidisci, però non molto frequente: presso Montorio (Seguier), nella Valle di Caprino (Pona), nei dintorni di Torri sul Benaco (Rigo!), in M. Baldo nella Valfredda (Pollin. herb.!), presso Cavalo alle falde del Pastello (Pollin. herb.!) ecc. Da maggio a giugno. 2

Gen. XV. - Botrychium.

48. Botryehium Lunaria Sw. in Schrad. Journ. Bot. II, p. 110, et syn. fil. p. 171; Pollin. viag. etc. p. 121, et fl. veron. III, p. 209; Bertol. fl. ital. crypt. pars I, p. 30; 
Vis. et Sacc. cat. etc. p. 9; Osmunda Lunaria L. sp. pl. ed. II, 1519; Lunaria racemosa ovvero minore del Matthioli Pona M. Baldo p. 195; Lunaria minore o racemosa del Matthioli Pona ibid. p. 240; Lunaria del graspo Calc. viag. etc. p. 12; Osmunda foliis lunatis Segu. pl. veron. I, p. 75; Moren. herb.!

Nei pascoli e nei prati dalla regione montana alla alpina: nel M. Baldo in Costabella!, Ortigara!, La Ferrara (Seguier), Valfiedda (Pollin. herb.!), 'La Corona (Pollin. herb.!), Novesa (Pona, Pollini), Altissimo di Nago! ecc.; nei Lessini lungo tutta la catena!, presso Velo (A. Massalongo in herb. Da Campo!), Spiazzoi!, le Scandole!, Tinasso! (e Seguier), nel M. Pastello (Pollini herb.!) ecc. Da gingno a settembre secondo la altitudine. 2

Nel por termine alla euumerazione delle Protallogame raccolte sino ad oggi nella Provincia Veronese, mi corre l'obbligo di manifestare la mia riconoscenza ai colleghi ed agli amici, i quali mi favorirono di esemplari appartenenti a questo interessantissimo gruppo di piante. Devo speciali ringrazimenti al Barone Vincenzo Cesati Direttore del R. Orto Botanico di Napoli, ed al Dott. G. Gibelli Prof. di Botanica nella R. Università di Bologna, i quali mi offrirono il mezzo ed il modo di superare non poche, nè lievi difficoltà, mettendo a mia disposizione i pacchi dei loro erbari, che contenevano i campioni autotipi delle specie e delle forme che maggiormente mi importava di esaminare. Se nelle future ricerche, nuove forme verranno ad accrescere la Flora così ricca e splendida di questa mia Provincia, sarà mia cura farne argomento di un'appendice al lavoro, del quale ho intrapreso la pubblicazione nel presente fascicolo del Giornale Botanico. 






\section{Al. Coscie ${ }^{m}$ Amies \\ Com. Arsinso te Betto \\ A. Gouni}

Estratto dal Nuvo Giornale Botanico Italiano Vol. XIV, N. 2, Aprile 18ז2.

\section{PRODROMUS FLORAE VERONENSIS, AUCTORE}

\section{A. GOIRAN (Continuatio).}

Divisio II.

\section{Phanerogamae,}

Sopra una superficie di chilometri quadrati 2747,54 fra la altitudine massima di m. 2200 alla punta del telegrafo, in M. Baldo, e la minima di circa m. 6-7 nella bassa pianura, la Provincia Veronese offre una flora fanerogamica, che nella regione veneta appena è inferiore per varietà e dovizia di forme a quella brillantissima dell' Udinese. La nostra zona botanica pertanto è in grado da gareggiare vantaggiosamente con altre che in Italia e fuori, sopra una estensione di suolo uguale se non superiore, si dimostrano maggiormente favorite. Tale ricchezza di vegetazione troviamo distribuita sopra un terreno, il quale sebbene geologicamente e geognosticamente non molto variato, offre però una struttura orografica, che nelle molteplici catenelle, nelle quali si scindono e si frastagliano i monti maggiori, e nei colli, e nelle convalli, e nelle rupi ora apriche, altravolta umide ed ombrose, e nelle antiche torbiere, e sulle sponde 
dei numerosi corsi d'acqua, e nei paduli non ancora redenti alla coltivazione, presenta svariatissime stazioni nelle quali ponno a tutt' agio annidarsi le molteplici forme che da epoca antichissima resero celebratissima la classica nostra flora.

La ossatura della Provincia veronese è essenzialmente calcarea. In ordine ascendente la sua stratigrafia si può compendiare nel seguente quadro:

(a) Giura

Lias (Dolomie)

$\left.\begin{array}{l}\text { Oolite inferiore } \\ \text { Oolite media }\end{array}\right\}$ (Dogger)

$\left.\begin{array}{l}\text { Oxfordiano } \\ \text { Kimmeridgiano } \\ \text { Titoniano }\end{array}\right\}$ (Giura superiore)

(b) Creta I Creta media marnosa

Senoniano (Scaglia rossa)

(c) Eocene - Formazione nummulitica.

E incerta la presenza dell Oligocene; controversa quella del Miocene: che secondo alcuni comparirebbe a Porcino verso la estremità meridionale del $\mathrm{M}$. Baldo, mentre l'amico e collega Enrico Nicolis, il quale $\mathrm{mi}$ ha favorito le note sulle quali ho compilato il presente schizzo geologico, sospetterebbe tale formazione debba ascriversi al Piano Eocenico di Priabona. Le brecciole e le tufe basaltiche ed i basalti compatti squarciano e frastagliano le formazioni mesozoiche e le coceniche; le alluvioni quaternarie compaiono nella Valle dell'Adige e nell'Agro veronese; anzi i conglomerati, le breccie e le puddinghe che si osservano nella prima, secondo il parere di illustre geologo, sarebbero da ascriversi al periodo pliocenico; i depositi morenici, ammantate le falde estreme del M. Pastello, dei due versanti del M. Baldo e dei monti minori e colli che stanno fra l'Adige ed il Benaco, si spiegano nel grandioso anfiteatro di Rivole di storica e preistorica memoria, nelle graziose collinette, nei talus di sfacelo e nei terrazzamenti che formano per intero l'Agro 
veronese, per iscomparire poscia più al basso, mascherati dalle alluvioni moderne e dall'humus fertilissimo, che forma fra l'Adige, il Mincio ed il Po la parte più ubertosa e feconda della Provincia.

Sarebbe per fermo argomento di studio sotto ogni aspetto interessante lo indagare, quale sia il coefficiente di importanza da attribuirsi alla natura litica e geologica del suolo, sulla distribuzione delle forme vegetali viventi nelle varie regioni. Nel lavoro, che offro ai lettori del Giornale Botanico, non ho minimamente la pretesa di toccare solamente tale scabroso argomento; mi limito ad alcune induzioni, le quali sembrano legittimamente scaturire dalle osservazioni fatte nelle mie escursioni. 亡 un fatto che la ricchezza della flora nostra posta a confronto della poca varietà dei terreni geologici, sembrerebbe dimostrare, che la natura di questi non abbia sulle forme vegetali che vi hanno dimora, una influenza assoluta; e tale illazione trovo validamente corroborata, vedendo che il M. Baldo da solo, fatta eccezione per le forme che richiedono luoghi e condizioni speciali non reperibili in quella catena, ovvero che, per circostanze non facilmente determinabili, si trovano astrette a stabile dimora in un dato punto ed entro uno spazio anche angustissimo, ha una flora che per numero e qualità quasi compendia quella della intera Provincia. Però non si può negare che alcune forme, a parità di ogni altra condizione esterna, dimostrano una marcatissima elezione per terreni speciali. Se lungo la catena delle Alpi vedo alcune specie mostrarsi esclusivamente, o quasi, nei terreni granitici, ed altre invece nei calcarei, presso di noi trovo la Poa minor Gaud. vivere preferibilmente nelle rupi dolomitiche elevate; nei detriti basaltici ho osservato crescere più lussureggiante e più copioso che altrove il Rumex Acetosella $L$., e fra i basalti soltanto, almeno sino ad oggi, ho raccolto il Prumus Chamaecerasus Jacq. ed il Trifolium hirtum All., due specie nuove per la flora veronese; la Scorzonera hirsuta $L$. è sempre stata trovata da me nei terreni morenici della Provincia a Rivole, presso Villafranca, nelle vicinanze di Guastalla; la Daphne alpina $L$. nelle rupi giurassiche, dalla Valle dell'Adige presso Ceraino (m. 107) alle vette del MI. Pastello (m. 1122), al Corno d'Aqui- 
lio (m. 1543), al Passo della Sega (m. 1490); e così di altre. E pure degno di nota che se prendiamo a considerare la distribuzione delle forme in ordine alle tre formazioni predominanti Giurassica, Cretacen, Eocenica, non possiamo non rimanere meravigliati di fronte alla non dubbia povertà numerica delle forme che crescono nella cretacea, paragonata alla abbondanza di quelle che osserviamo nelle altre due. Ora questa povertà è dessa una reale conseguenza della semplice natura geologica del terreno? o, più razionalmente, della scarsezza di alimenti plastici ed assimilabili? della struttura fisico-molecolare dei materiali litici e del modo loro di comportarsi di fronte alle energie ed alle condizioni esterne? e quando vediamo che la Flora fossile della Creta, almeno nel versante meridionale delle Alpi, è poverissima se non nulla, mentre ricchissime in alcuni punti ci si presentano la Giurassica e la Eocenica, dobbiamo vedere nel fatto attuale la continuazione di un fenomeno che risale alle epoche geologiche? e la stessa esuberanza di vegetazione che presenta il M. Baldo non potrebbe avere una qualche relazione coi fenomeni sismici che da epoca immemorabile, e talvolta con periodi prolungati, tormentano quel monte? i quali fenomeni facendo variare la temperatura del suolo ed insinuando in questo anidride carbonica e vapore acqueo diventano in certo qual modo i fattori degli elementi più essenziali alla vegetazione?..... Tutto sommato inclino a ritenere, che nel fissare le stazioni e le abitazioni dei tipi e delle specie, la natura geologica del suolo, presa nel senso stretto della parola, ha soltanto una influenza relativa; la quale viene limitata a ciò, che certe forme potranno abitare esclusivamente o preferibilmente taluni terreni o roccie, perchè in queste trovano, secondo ogni probabilità, principî od elementi indispensabili al loro mantenimento ed alla loro conservazione. In quella vece le condizioni fisiche tanto interne che esterne, la natura idrologica della stazione, il grado di compattezza, di coesione, di permeabilità e di tenacità del terreno, la sua conduttività termica, il potere assorbente per il calore, la distanza delle temperature estreme, la esposizione, la altitudine, ed anche nella estensione relativamente piccola di una data zona la stessa latitu- 
dine, la orografia della regione, la natura, il modo, il grado della coltivazione, sono elementi tutti che indubbiamente fanno sentire la loro influenza nel caratterizzare le singole flore nello spazio e nel tempo, e contribuiscono potentemente alla distribuzione ed alla localizzazione delle forme, che nell'attuale periodo tellurico sono la manifestazione della vita vegetale. La quale distribuzione e localizzazione considero quale corollario di un postulato, che ritengo evidente: vale a dire che - nelle stazioni le quali presentano identiche condizioni interne ed esterne crescono o per lo meno possono crescere forme identiche; postulato che non solo vale a rendere ragione delle somiglianze e delle discrepanze che riscontransi nelle flore delle varie regioni, ma delle variazioni benanco che si osservano di anno in anno nelle flore locali, sia colla scomparsa di alcune forme, sia con la apparizione di altre non prima viste, e trasportatevi o per caso fortuito, ovvero per opera di alcuno dei tanti mezzi di disseminazione o anemofila o idrofila o zoofila, dei quali si vale la natura.

Sotto questo punto di vista la Provincia veronese si trova in condizioni favorevolissime. La catena del M. Baldo (Alpi Retiche) sulla destra dell' Adige, i Monti Lessini e lo sviluppo del gruppo del M. Posta (Alpi Carniche) sulla sinistra del fiume, formano un vasto arco parabolico che a NW, $\mathrm{N}$, NE accerchia la parte superiore della Provincia. Da questa barriera si staccano le catene secondarie ed i gruppi minori, che correndo approssimativamente da $\mathrm{N}$ a $\mathrm{S}$ ed in mille modi frastagliandosi e dividendosi determinano, in una alle collinette moreniche che formano ad W e SW l'Agro veronese, un vasto anfiteatro nel quale si adagia la nostra pianura. Le giogaie alpine pertanto valgono a moderare nei loro effetti le fredde correnti del primo e del quarto quadrante, le quali appena ne adustano i picchi e le creste più elevate; mentre i colli ed i monti minori sono convenevolmente orientati per risentire l'influsso delle altre più tepide e miti che spirano dal secondo e dal terzo. Per buon tratto dell'anno le cime più ardite delle nostre prealpi sono totalmente spoglie da nevi, le quali oggidì ad estate un po'inoltrata, a stento si mantengono con qualche 
rara falda in talune fra le valli alpine più elevate, che squarciano il fianco occidentale del M. Baldo, ovvero fra gli scoscesi dirupi del M. Posta; la orientazione apprcssimativamente da $\mathrm{N}$ a $\mathrm{S}$ delle ultime diramazioni delle prealpi, costituenti la frastagliatissima rete delle colline veronesi, rende queste sommamente atte a risentire la benefica influenza delle radiazioni solari; la immensa copia infine delle acque adunate nel vasto bacino del Benaco, trasforma questo in un vero serbatoio di calore, che mentre contribuisce a mantenere lunghesso quella riviera fortunata una eterna primavera, fa sentire benanco nelle zone finitime il suo influsso moderatore. Nel complesso tutte queste circostanze concorrono a determinare condizioni climatologiche oltre ogni dire dolci e temperate; e non verificandosi mai, a meno di anni eccezionali e fortunatamente a lungo periodo, temperature estreme così discoste, da caratterizzare i climi che dai meteorologisti sono detti continentali, noi possiamo asserire che, almeno nell'alta pianura e nei colli, godiamo di un clima se non marino, per lo meno prossimo assai a quello di altre stazioni più marittime della nostra. Quindi nella distribuzione topografica delle forme costituenti la flora nostra, fatta astrazione da quelle che come la Campanula ramosissima Sibth. et Sm. (C. Loreii Pollin.), o la Eleocharis atropurpurea Koch, o il Nardurus tenellus Rchb. ecc. si trovano circoscritte entro un' area limitatissima, ovvero esigono condizioni speciali come Stratiotes aloides L., Aldrovanda vesiculosa L., Littorella lacustris L., Ammannia verticillata Lam., Hibiscus roseus Thore ecc., troviamo predominante la influenza della altitudine: che è quanto dire della esposizione alle correnti superiori della atmosfera ed alle radiazioni solari, e forse alle azioni delle correnti elettriche telluricoatmosferiche, che sulle creste dei monti devono manifestarsi più vigorose che non nelle stazioni più basse. Mentre le rupi elevatissime, le praterie, i pascoli, gli ericeti ed i dumeti delle parti più alte ricettano gran parte dei generi e delle specie che allignano lungo tutta la zona alpina delle Alpi Italiane, i colli e le ime pendici dei monti sono rivestite da una vegetazione che si avvicina a quella della Liguria e della Toscana. Le creste del M. Baldo, le scoscese valli ed 
i torrenti che ne solcano il versante prospiciente il Benaco, accolgono forme prettamente alpine; che in parte si incontrano pure nei Lessini, nel M. Posta, nel M. Campobrun, alla Lora, nel M. Zeola, nel M. Alba ecc., e tra le più caratteristiche od insigni citerò: Festuca pumila Vill., Poa minor Gaud., Agrostis rupestris All., Carex firma Host, Lloydia serotina R.chb., Chamaeorchis alpina C. L. Rich., Papaver pyrenaicum DC., Arabis coerulea Haenlee, Petrocallis pyrenaica $R$. Br., Draba stellata Jcq., Hutchinsia alpina R. Br., Cerastiun latifolium L., Silene acaulis L., Cherleria sedoides L., Geraniun argenteum L., Saxifraga androsacea L., Soldanella minima Hoppe, Salix reticulata $L$., Salix retusa $L$., Salix herbacea $L$. ecc. Le ime pendici dei monti che corrono alla pianura o alle sponde Benacesi offrono asilo a numerose varietà dell' Olea europuea $L$. ed all'Amygdalus communis $L$., coltivati sopra vasta scala, mentre nei giardini ed attorno alle eleganti ville crescono lussureggianti il Pinus halepensis Mill., il Rhamnus Alatermus $L$., l'Hyacinthus orientalis $L$. ecc., asportati da regioni più meridionali della nostra; sulle sponde incantevoli del Benaco tra Garda e la punta di S. Vigitio il Laurus nobilis L. è oramai quasi inselvatichito, qualche volta l'Agave americana $L$. innalza i suoi fioriti candelabri, vivono il Crataegus Pyracantha Pers., la Plitllyrea variabilis Timb., e tra i crepacci delle roccie compare l'Olea europaea var. Oleaster Hoffm., mentre al disopra di Torri il Cistus albidus $L$. fa pompa delle fugaci ed incarnate corolle; nelle fessure delle rupi, nelle morene o nelle alluvioni, nelle siepi, nei muri allignano: Rhus Cotinus L., Zizyphus vulgaris Lmk., Paliurus australis Lmk., Punica Granatum L., Pistacia Terebinthus L., Jasminum officinale L., Quercus Ilex L., Capparis rupestris Sibth. et Sm., Capparis spinosa L., Centranthus ruber DC., Farsetia clypeata R. Br., Arabis muralis Bertol., Asparagus acutifolius L., Asphodelus fistulosus $L$. ecc.; nei campi coltivati, nei prati, negli argini, nei pascoli, negli stessi fossi della città si incontrano non poche stirpi segnalate in altre stazioni più meridionali, e tali sarebbero Erianthus Ravennae Palis. de Beauv., Allium neapolitanum Cyr., Campamila Erimus I., Trixago latifolia Richb., Spartium junceum L., Ononis reclinata L., Smymium Oiusatrum L., Erodium malacoides Willd., Erodium moschatum L'Herit., 
Ranunculus velutinus Ten. ecc.; sporadiche infine o subspontanee o avventizie non poche specie dimostrano colla loro presenza la attitudine del nostro suolo ad albergare le forme che crescono di preferenza nelle zone più vicine al mare, e come tali nomino Nigella damascena L., Glaucium luteum Scop., Bupleurum protractum LnT, Nardosmia fragrans Rchb., Cupularia graveolens Godr. et Gren., Nicandra physaloides Gärtn., Vitex Agnus-Castus L. ecc.

Nelle specie enumerate ho voluto in certo qual modo stabilire i limiti fra i quali oscilla la nostra flora. Entro questi termini estremi vivono e lottano centinaia di altre specie, ora generalmente diffuse e più o meno sparse e frequenti, altra volta localizzate, rare o rarissime. Ma nel sostratto che raccoglie la posta maggiore nella somma delle Fanerogame veronesi, non tardiamo a sorprendere un fenomeno rimarchevolissimo già accennato da me in altre scritture. Il quale in ultima analisi si riduce ad una continua oscillazione nel numero delle specie che rappresentano la flora attuale, nella scomparsa o nella emigrazione di taluna di esse, nella immigrazione incessante di altre, ad un viaggio o movimento di forme che, o preesistenti sembrano quasi circolare frammezzo a quelle che si dimostrano più stabili ovvero più volgari e diffuse, oppure importate da circostanze di natura e di indole diversa, appaiono d' un tratto fra le prime per prendervi stanza, effimera qualche volta, duratura frequentemente; recando per tal modo modificazioni sensibili e continuate nella flora della regione.

\section{Classis I.}

\section{Gynospermae.}

Dei cinque ordini appartenenti a questa classe, sono rappresentati nella nostra flora i due soltanto delle Taxaceae e delle Pinaceae. Delle Gnetaceae, cresce nel Tirolo meridionale la Ephedra distachya L. (E. vulgaris a subtristachya C. A. Meyer), sulle rupi del Doss' di Trento, a Schlanders nella Valle Venosta, a Siegmundsliron presso Bolzano: ma questa specie non è stata sino ad oggi rinvenuta nel Veronese, nè, per quanto io mi sappia, in alcun'altra parte del Veneto, nè nel Bresciano. Si coltivano nei giardini alcune specie dell'ordine delle Cycadaceae. 


\section{Ordo V.}

TAXACEAE.

Non cresce presso di noi che il Taxus baccata $L$.; unico rappresentante dell'ordine e del genere, non solo in Italia, ma in Europa.

Gen. unicum. - Taxus.

49. Taxus baccata $L . s p . p l$. 1472; Pollin. viag. p. 107, 120; fl. veron. III, p. 193, et herb.!; Bertol. fl. it. X, p. 388; Ambros. fl. Tir. austr. II, p. 28; Parlat. fl. it. IV, p. 95; Vis. et Sacc. cat. p. 65; Nomi volgari adoperati in Italia a designare le principali piante di bosco in Ann. Minist. Agricolt. etc. p. 180; Goir. erb. forest. veron. p. 103; Taxus arbor Calc. iter in Bald. p. 23; Tasso detto dal Lobelio Milos di Theofrasto ovvero $\delta \mu i \lambda x \xi$ di Dioscoride et di Galeno Pona M. Baldo descritto p. 152, e nell' indice dell'opera Tasso arbore; Taxus Segu. cat. pl. p. 98, et pl. veron. II, p. 262; Moren. herb.! - Ternacolo: Nasso, nome antichissimo ricordato dal Seguier, Nas, Tasso!, e presso Lughezzano e Squaranto nei Lessini Mazzacaval!. - Icon.: Rchb. ic. fl. germ. et helv. f. $114 \%$.

Il Tasso presso di noi è per lo più allo stato di frutice, ma può assumere le dimensioni di un albero, e tale diventa quando sia coltivato; non è molto frequente, e per lo più si incontra sporadico dalla regione montana e subalpina, ove predilige $\mathrm{i}$ boschi e le rupi ombrose, ai colli e quasi alla pianura. Il Pona lo osservò nella Valle di Caprino dove, diventato rarissimo ai tempi di Seguier, forse oggidì si trova appena coltivato nei giardini ; Calceolari in M. Baldo alla Valle dell'Artillon ove è pure indicato dal Pollini; Seguier ai confini del Veronese col Trentino, presso Cassone alle falde del M. Baldo, ed ai confini della Provincia veronese col Vicentino verso Volpiana nelle vicinanze di Bolca; il Pollini nel M. Baldo nella Valle dell'Orsa presso la Ferrara e nella antica selva di Malcesine. Io lo ho raccolto nel M. Baldo nelle selve presso le Acque negre! e le Fassole!; nei M. Lessini presso Lughezzano nel luogo detto la Valle!, in vicinanza di Squaranto!, nella Valle dei Ronchi!, ove è segnalato benanco dallo Ambrosi, presso Avio! nella Valfredda al piede della salita al passo della Sega ore cresce copiosissimo in societì con l'Ilex Aquifolium; infine a breve distanza dalla città di Verona nelle 
convalli dei monti e colli prossimi ad Avesa, p. e. nel Vajo del Borago!, presso il luogo detto il Salto fra il Maso e Costagrande, ed anche più a basso. Nelle Reliquie botaniche del prof. A. Massalongo ho trorato un esemplare raccolto presso Velo (M. Lessini), $\theta$ nella scheda si legge in nemore dicto dei Croci in quo una planta tantum adest: nella stessa scheda è detto che è frequente presso Selva di Progno, nella Valle d'Illasi. Nel complesso possiamo dire che vegeta spontaneo fra $1300 \mathrm{~m}$. e meno di $150 \mathrm{~m}$. di altitudine, trovandosi distribuito a sbalzi sopra di una linea che corre dal M. Bolca al Benaco, dalla quale accenna a muo. versi verso la pianura. - Fiorisce al principio della primavera ed anche sin dall'inverno se questo è mite: i frutti sono maturi in settembre ed ottobre. 5 - Per la bellezza della chioma e del portamento il Tasso viene coltivato in quasi tutti i nostri giardini nei quali cresce lussureggianı́e, tanto nel piano che nei colli e nei monti; ricordo il bellissimo esemplare che può osservarsi nell'Agro Veronese a Guastalla nella villa del conte Giulio Giusti: ha una altezza totale di 10 metri, presenta al piede una circonferenza di metri 3,83 , e porta 20 rami principali i quali misurano alla Joro base una circonferenza che oscilla fra met. 0,58 e m. 0,29. Nell' Erbario Polliniano si trovano due esemplari; il primo proveniente da pianta coltivata, il secondo raccolto in M. Baldo al Prà di Brentonico!.

\section{Ordo VI.}

P INA C E A E.

L'ordine delle Pinaceae, per le condizioni fisiche della regione, conta nella flora veronese poche specie soltanto dei generi Pinus e Juniperus; la metà appena, considerate complessivamente, di quelle che nascono sul suolo della Penisola italiana. Non parlo dej generi Cupressus e Thuja, perchè le tre specie ad essi appartenenti, che vivono presso di noi, ncn solamente non sono indigene, ma appena oso dichiarare inselvatichite: in verità non mi sono mai imbattuto in alcun individuo che si potesse chiamare con certezza subspontaneo, ovvero riconoscere come nato per disseminazione dalla pianta madre. Nel genere Pinus la natura si mostrò avara, limitando soverchiamente presso di noi il numero delle forme: l'opera inconsulta di distruzione delle generazioni che ci hanno preceduto, ha per ognuna di esse ridotta a minime proporzioni l'area di vegetazione. Non sono convinto che le norme e le discipline, le quali reggono in oggi nel Regno d'Italia la economia forestale, sieno sufficienti ad impedire lo sperpero del poco che rimane. 
Tribus 1. - Abietineae.

Genus I. - Pinus.

Sub-genus 1. - Pinus Endl. conif. p. 13\%.

Sectio 1. - Pinea.

50. Pinus sylvestris Segu. cat. p. 82, et pl.veron. II, p. 255; Moren. herb.!; L. sp. pl. 1418, excl. varietatibus; Pollin. viag. p. 107, fl. veron. III, p. 136 et herb.!; Bertol. fl. it. X, p. 25\%; Ambros. fl. Tir. austr. II, p. 9, excl. syn. P. rotundatae Link, P. uliginosae Neum., P. obliquae Saut.; Parlat. fl. it. IV, p. 46; Vis. et Sacc. cat. p. 65; Nomi volgari etc. p. 106; Goir. erb. forest. ver. p. 69; P. Mugus Jacq. icon. rar. I, p. 193; Pino Calc. viag. di M. B. p. 12 ; Pona $M$. B. p. 225; Pino silvestre montano Pona M. B. p. 13. Vernacolo: Pin, Pigno, Pigno salvadego, Pignolar salvadego (Monti), Pin nero!, Pino!: quest' ultimo nome è pure ricordato dal Seguier nella sua classica opera Plantae veronenses. Gli strobili sono chiamati Pigne o Pine, e nei M. Lessini si indicano benanco col nome di Cotai!: tali denominazioni appartengono pure nel linguaggio volgare alle altre specie del genere, nè quindi ne farò ulteriormente menzione. - Icon.: Rchb. Icon. fl. germ. et helv. f. $112 \%$.

Frequentissimo. Il Seguier lo indica soltanto nel M. Baldo presso Malcesine: ma sporadico in tutti i colli veronesi e benanco nella pianúra, si spinge da questa a tutta la regione montana e penetra nella subalpina, ove diventa assai raro e viene sostituito dal Mugo. Nei monti si trova frequentemente isolato, altra volta vive in società con il Larice, col Picea, con l'Abete comune ed anche con la Quercia: qua e là forma boschetto da se. Nei monti di Valpantena!, Tregnago!, etc. nel M. Bolca! nel M. Pastello!, S. Anna!, Erbezzo!; in tutta la catena dei Lessini!, e del Baldo!, dalle radici a $1200-1300 \mathrm{~m}$. di altitudine. Il legno del Pino si adopera presso di noi come materiale da costruzione e come combustibile; serve a fabbricar carbone: coi rami si coprono o si fabbricano le capanne dei pastori: è pure coltivato nei parchi e nei giardini. 5 Albero più o meno elevato: qualche rara volta si incontra allo stato di frutice; fiorisce in aprile e maggio. 
Nel Nizzardo, lungo la pittoresca strada della Cornice, ho raccolto il P. sylvestris alle falde del colle di Montgros a breve distanza ed a poca altezza sul mare: cresceva in società col P. halepensis Mill. e poco discosto dal P. Pinaster Soland.

51. Pinus Mugus Scop. fl. carn. ed. 2, p. 247; Pollin. fl. veron. III, p. 135, n. 1902; Bertol. fl. it. X, p. 258; Ambros. fl. Tir. austr. II, p. 11; Vis. et Sacc. cat. p. 65; Nomi volg. etc. p. 102 ; Goir. erb. forest. veron. p. 67; P. montana Duroi obs. bot. p. 42; Parlat. fl. it. IV, p. 49; P. Pumilio Haenle Beobacht. aut Riesengeb. p. 68; Poilin. viag. p. 51, 60, 100, 105, 121 et herb.!; Picea silvestre detta dagli abitanti Mughi Calc. viag. di M. B. p. 13; Pino Tubulo di Plinio, genere di Pinastro sterile, gl' Italiani lo chiamano Mugo Pona M. B. p. 196; Pino o Mugho del volgo, ovvero Pino Tubulo di Plinio o genere di Pinastro sterile Pona l. c. p. 237; Pinus sylvestris altera Segu. cat. $p l$. p. 82 ; Pinus sylvestris montana altera Segu. pl.veron. II, p. 256 ; Moren. herb.! - Vernacolo: Mugo, nome antichissimo, Muga, Bugo, Mughi, Mughe, Bughe, Pino salvadego. - Icon.: Rchb. icon. fl. germ. et helv. t. 1130.

Il Mugo cresce abbondantemente nel Baldo tanto sul versante orientale che sul fianco prospettante il Benaco, fatta eccezione dalla estremità più meridionale: può dirsi sia una delle piante caratteristiche della catena. Partendo dalla Valfredda e Naole (1200-1500 m.) si innalza alle creste più elevate $(2000-2200 \mathrm{~m}$.): presso lil Val brutta e la Lonza, $\Theta$ sotto il Coval Santo si possono oggidì ancora osservare estese e rigogliose mugaie. Si interna pure in tutte le valli che solcano il versante occidentale, nel quale scende al disotto dei 1000 m.: molto improvvidamente, sono alcuni anni, nella Valle delle pietre sono stati tagliati alcuni boschetti che ricoprivano quelle scogliere. Si trova pure sparso, forse meno abbondantemente, in tutta la catena dei Lessini!, sempre bene inteso nella zona più elevata; cresce benanco nel $M$. Posta!, Campobrun!, Zeola!, Alba! etc. Si adoperano i rami del Mugo come combustibile o per fabbricar carbone, per costrurre capanne etc.: di essi oggidì ancora, come ai tempi di Seguier (pl. veron. p. 256), incolae ligamina parant ad dolia vincienda.

Fiorisce da maggio a luglio secondo le stazioni. 5 - La forma che cresce nella nostra zona corrisponde al $P$. Pumilio Huente, così ben descritta dal Seguier $l$. c., quando la dice Pini species pumila.... quae statim a radice in lentos et obsequentes ramos, tametsi crassos dividitur, parum se extollentes, sed longe lateque sese diffundentes. Alcuni individui però, nelle stazioni più basse, accennano ad avvicinarsi alla forma cor- 
rispondente al $P$. uncinata $R a m$. In quei luoghi nei quali le nevi rimangono più a lungo ed in maggior copia, come nelle valli alpine del Baldo prospicienti il Benaco, sul M. Posta ecc., i rami del Mugo, gli inferiori specialmente, sono più lungamente repenti e quasi aderenti al suolo, per effetto sicuramente della pressione e del scivolamento della massa soprastante. - Ho scritto Mugus e non MIughus, ritenendo giuste e razionali le considerazioni che a proposito della ortografia di questa parola sono fatte dal dott. Francesco Facchini (in Ambr. fl. Tir. austr. II, pag. 117).

\section{Sectio 2. - Cembra.}

52. * Pinus Cembra L. sp. pl. 1419 ; Pollin. fl. veron. III, p. 137; Ambros. fl. Tir. austr. II, p. 14; Vis. et Sacc. cat. p. 66; Nomi volg. etc. p. 100; Goir. erb. forest. veron. p. 66; Pinaster Micheli nov. pl. gen. p. 223. - Vernacolo: Zimbro, Zirmo, Zirmolo, Cirmolo.

Stando alla testimonianza del Micheli $l$. c. e del Laicharding ( $A m b r$. op. cit. p. 15), sembrerebbe che una volti questa specie crescesse sul M. Baldo: tale illazione sarebbe confermata dal trovarsi perpetuato nel dialetto nostro il nome volgare della pianta. Però nè il Calceolari, nè il Pona, nè il Seguier, nè il Moreni accennano solamente ad essa, come posteriormente non la rinvennero nè il Pollini, nè il Tonini nè, per quanto io mi sappia, alcuno dei tanti botanici che perlustrarono il Veronese; anche Frate Fortunato da Rovigo, nell'elenco ms. delle piante che crescono sul Monte Baldo, e che ra unito al suo copiosissimo erbario esistente nel Civico Museo di Verona sotto la intitolazione Theatrum plantarum, non ne fa cenno; ne ho trovato un esemplare nelle Rcliquie botaniche di A. Massalongo, ma proveniente da pianta coltivata, perchè nella scheda si legge ex meo viridario. Del resto il nome vernacolo non sarebbe neanco argomento sufficiente per affermare, che effettivamente il C'embro crescesse in epoca remota sul M. Baldo: perchè dei quattro noni sopra citati, il quarto Cirmolo, non è che pura corruzione di Zirmolo, e gli altri tre sono quelli stessi coi quali questa conifera è chiamata nel vicino Trentino. Ora per le sue buone qualità il legno del Cembro viene adoperato per diversi lavori; laonde la pianta ha un valore artistico e industriale che, greggia o lavorata, la fa argomento di esportazione. Verona, come accenna il Seguier, è stata in tutti i tempi un emporio sul quale si versano i legnami provenienti dal Tirolo: il Cemuro importato per tal modo sul nostro mercato, ha lasciato nel dialetto. quei nomi istessi coi quali era designato nel luogo suo di origine. Ad ogni modo, dato anche che secoli addietro il $P$. Cembra crescesse sul M. Baldo, possiamo asseiire che oggidì è totalmente scomparso. Si coltivava e si coltiva tuttora nei giardini: potrebbe tentarsene la piantagione nel rimboschimento dei nostri monti. - L' esemplare di P. Cembra esistente nell' Erbario Polliniano proviene dalla Val di Fassa. 
Sub-genus 2. - Sapinus Endl. conit. p. 82.

Sectio 1. - Larix.

53. Pinus Lavix L. sp. pl. 1420; Pollin. viag. p. 107; fl. veron. III, p. 138, et herb.!; Bertol. fl. it. X, p. 268; Parlat. fl. it. IV, p. 59; Vis. et Sacc. cat. p. 66 ; Nomi volg. etc. p. 101; (7xoir. erb. forest. veron. p. 66; Larix europaea $D C$. fl. fr. III, p. 277; Larice Calc. viag. di M. B.p. 12; Pona M. B. p. 23s; Larix folio deciduo, conifera Segu. cat. pl. p.60, et pl. veron. II, p. 257; Moren. herb.! - Vernacolo: Larese, nome antichissimo ricordato da Seguier, Larzo, Arese, Ares, Areso. - Icon.: Rchb. ic. fl. germ. et helv. f. $113 \%$.

Il Larice si incontra si può dire in tutti i nostri monti, ma non vi è copioso, e dal limite dclla zona della Vite sale alla regione subalpina; ora è isolato, ovvero in società con il Pinus Picea e il P. sylvestris, ora forma qualche volta piccoli boschetti da sè. Il Seguier scriveva di questa conifera ( $p l$. verón. p. 257) " in montibus Lessinensibus mibi interdum * occurrit, pumila tamen ceterarum coniferarum instar, quae in agro "veronensium proveniunt, ita ut nullius sint usus "; non fa cenno della presenza del Larice nel M. Baldo, e ne arguisco che ai suoi tempi questa pinaceil vi fosse assai rara. Ogggidì le cose stanno negli stessi termini: nei Lessini e nei monti che ne dipendono si trova qua e là; a Lavel! e Foldrona! presso Cerro; Prati! presso Lugheazcino; Ormi! presso Chiesanova; S. Anna!; Fosse! etc.; fra Bolca e S. Bartolomeo!; nel MT. Tondo! fra le valli Policella e Pantena in società col P. Picea e $P$. sylvestris, e si può dire assieme all'Olivo, alla Vite, al Castagno, al Gelso. 亡̀ meno frequente nel M. Baldo, ove lo ho osservato alla Ferrara, e più in alto nella catena, all'Artillon ove è pure indicato da Calceolari e Pona. Sporadico si trova pure nei colli; viene infin? coltivato nei giardini. 5 - Fiorisce in aprile e maggio.

\section{Sectio 2. - Picea.}

54. Pinus Picea Duroi obs. bot. p. 37; Parlat. fl. it. IV, p. 62; Vis. et Sacc. cat. p. 66; Nomi voly. etc. p. 103; Goir. crb. forest. veron. p. 68; P. Abies L. sp. pl. 1421; Pollin. viag. p. 107; fl. veron. III, p. 150, et herb.!; Bertol.fl.it. X, p. 272; Abies Picea Ambros. fl. Tir. austr. II, p. 18; Picea Calc. viag. di M. B. p. 12; Avezzo Pona M. B. p. 225; Abies 
tenuiore folio fructuque deorsum inflexo Segu. cat.pl.p. 5, et pl. veron. II, p. 254; Moren. herb.! - Vernacolo: Pezzo, il nome antichissimo ricordato da Seguier, Pezzi (Pollin. herb. in scheda!), Abete o Abeto rosso!, Avezzo!. Nel dialetto cimbro della Giazza il Pezzo porta il nome di Tamne al singolare e Tannan al plurale; in alcuni documenti antichi ricere pure la denominazione di Tande (fratelli Cipolla). - Icon.: Rchb. ic. fl. germ. et helv.f. 1138. " Arbor; raro frutex. Duae formae occurrunt. »

$\alpha$ crassifolia. - - Foliis crassioribus tetragonis, mm. 16-17 " longis, mm. 1, 5-2 latis, confertis, undique versis. "

$\beta$ tenuifolia. - Pinus baldensis Zuccagni cent. I obs. bot. in collect. J. J. Roem. p. 158-159. - . Foliis intensius viri" dibus, tenuioribus, mm. 8-15 longis, mm. 1-1, 5 latis; subtea tragonis, utrinque carinatis, in suprema ramulorum super" ficie confertissimis et rectis, ad latera ramulorum incurvis, - in inferiore distichis. Rami juniores inferne nudi, vetustio- res autem foliis rarioribus, adpressis sulummodo vestiti - apparent. Ceterum, formis intermediis, transit in var. $\alpha_{0}$ 。

In tutti i monti veronesi crescono tanto la varietà $\alpha$ che la var. $\beta$; la seconda forse è più frequente della prima. In alcuni punti dei Lessini, e nelle parti del M. Baldo che confinano col Trentino, il Picea forma ancora foresta; sporadico s'incontra ovunque in tutta la zona montana, nei colli e benanco nella pianura, innalzandosi per tal modo da questa sino a circa $1800 \mathrm{~m}$.; vive frequentemente in società con il $P$. Abies, P. Mugus, P. sylvestris. - Fiorisce da marzo a giugno secondo la altitudine. 5

Il P. baldensis di Attilio Zuccagni, che forse è la pianta del Seguier, come apparirebbe dalla frase Abies tenuiore folio, è stato primieramente segnalato dal Monaco Bruno Tozzi da Vallombrosa, il quale dal M. Baldo lo recò in Toscana sotto la denominazione di Abies germanica: esso si distingue a primo aspetto dalla var. $\alpha$, perchè più delicato di questa in tutte le sue parti, per le foglie più minute e sottili, appena bicarenate non tetragone, distintamente ed elegantemente distiche e disposte a mo di petline nella parte inferiore dei rami giovani, che quivi appaiono nudi. Ciro Pollini nella Flora, ritiene il $P$. baldensis quale semplice sinonimo del suo $P$. Abies, che è il vero $P$. Picea: però nel viaggio a p. 107 aveva riconosciuto che era una varietà, sebbene di lievissimo momento, di questo: per parte mia, essendomi proposto di enumerare nel presente lavoro le varie forme vegetali che crescono in questa regione, ho creduto dover ritenere lia pianta del Tozzi e dello Zuccagni come distinta varietà, sebbene dal Bortoloni e dal Parlatore sia stata fusa nella specie. - Nei 
nostri monti è pure frequente una forma elegantissima, la quale ha la massima parte dei rami, gli inferiori specialmente, distintamente e lungamente pendenti: p. e. a Revolto, presso le Scandole, i Merli ecc. nei Lessini, e nella parte del Baldo che confina col Trentino.

Sono noti i vari usi ai quali serve il Pezzo, che per il magnifico portamento è pure coltivato nei giardini e nei parchi. Il Seguier scrive che ai suoi tempi, una gran quantitì del materiale ricavato dal tronco del Picea, dalle Alpi Retiche veniva trasportats a Verona, * ex qua tam« quam emporio ad circumvicinas Italiae regiones transportatur. " Tale commercio, non so se in eguali proporzioni, continua pure oggidì, sia per trasporto ferroviario, sia per mezzo di zattere sull'Adige.

\section{Sectio 3. - Abies.}

55. Pinus Abies Dur. obs. bot. p. 39 ; Parlat. Al. it. IV, p. 66 ; Vis. et Sacc. cat. 2. 66; Nomi volg. etc. p. 9s; Goir. erb. forest. veron. $p$ 65; P. Picea L. sp. pl. 1120; Pollin. viag. p. $10 \%$; fl. veron. III, p. 13\%, et her.t.!; Bertol. fl. it. X p. 270; Abies vulgaris Ambros. fl. Tir austr. II, p. 16; A. taxifolia Desf. cat. p. 356 ; Abete Calc. viag. di $I I$. B. p. 12; Abiete Pona M. B. p. 237; Abies conis sursum spectantibus sive mas Segu. cat. pl. p. 5, et pl. veron. II, p. 253 ; Moren. hert.! - Vernacolo: Avezzo, Avezzi (Pollin. herb. in scheda!), Abete o Abeto bianco!, Daza: - Daze etiam vocantur a rusticis qui Avium vicum et circumvicina loca incolunt »(Sequier $7 . c$.$) . Osservo che$ nei Lessini si da più propriamente il nome di Daze, anzichè alla intera pianta, ai rami che, come è noto, sono distribuiti in giro da 3 a 5 attorno al tronco primario. Icon.: Rchb. ic. fl. germ. et hel,v. f. 1139.

Vive mescolato al precedente nella zona montana elevata e nella subalpina, però è di gran lunga più raro. Calceolari e Pona lo segnalarono all'Artillon di M. Baldo; e Seguier scriveva : " in confinibus agri vero" nensis cum Tridentino provenit, rara tamen, nec nisi sparsim, si " excipiantur eae, quae promiscue cum Piceis extant in declivitate Baldi

" montis prope Malsesine vicum, et quas indigenis excidere non licet " nisi ad damnum resarciendum propriae domui casu quodam allatum. » Pl. veron. II, $p$. 254. Oggidì troviumo ancora la Daza rella parte più settentrionale del M. Baldo; e nei Lessini presso la Sega!, al passo della Vecetta!, a Revolto!, alle Pozzette presso le Scandole!, a Campo rotondo!, Spiazzoi!, alla Tommasola presso il lago Boaro fra Erbezzo e Chiesanova!, nella Vallina presso Erbezzo! ccc. - Fiorisce da aprile a 
giugno secondo la altitudine e le condizioni della stazione t̀ anche coltivata come pianta di ornamento nei parchi e nei giardini. 5

Gli esemplari di P. Picea e P. Abies che si trovano nell' Erbario Polliniano provengono dalla antica Selva di Malcesine, ridotta oggidì a proporzioni meschinissime, a simulacro appena dello antico splendore. Il Pollini nella frase diagnostica del $P$. Abies (P. Picea fl. veron.), dice che le foglie in questa specie sono smarginate : tale carattere però non è costante, trovandosi sullo stesso ramo foglie smarginate, ottuse, tronche, ottusamente mucronate.

Le maestose selve di Larici, di Pini, di Abeti e di Faggi che un tempo vestivano $\mathrm{i}$ nostri monti furono in questi ultimi secoli al tutto divelte: sorgente fatale, esclamava Ciro Pollini, dellincostanza del nostro clima, dello straripamento dei fumi, e d'altri danni gravissimi (viag. p. 54). Attualmente la Provincia, il Comizio agrario, i Comuni, i privati dimostrano di voler pensare sul serio all'opera del rimboschimento, e procurano di conservare quel poco che ancora rimane delle antiche ricchezze forestali. Nè mancano esempi generosi, ai quali si augurano molti imitatori. Sino dal 1874 il marchese Ottavio di Canossa in Ime e Val Andrine di M. Baldo, ha fatto un primo esperimento, collo scopo di vedere se si potesse tentare con frutto il rimboschimento di alcuni tratti del M. Baldo che a tal uopo si riconoscessero più adatti. Vennero pertanto piantate in diverse stazioni 398 piante di Abete, provenienti 158 da un vivaio di Vicenza, e 240 dai dintorni di Chiesanova nei Lessini. Nell'antunno del 1878 si fece una piantagione di Larici e, in via di esperimento, di cinque Cedri Deodara; nel 1879 la piccola colonia, sempre per prova, si è accresciuta di alcune piante di Pinus austriaca e Cryptomeria Lobbiana. Sopraggiunto l'inverno rigorosissimo del 1879-80, il quale fu fatale presso di noi a tante piante, perirono le Cryptomeria, e soffrirono non poco i $P$. austriaca; i Deodara non risentirono danno alcuno ed oggidì sono sani e robustissimi; i Larici poi e gli Abeti manifestano i segni più evidenti di una esuberante e rigogliosa vegetazione. Alcune piantagioni sono pure state fatte alla Ferrara di M. Baldo dal sig. Candelpergher, le quali hanno sortito esito eccellentissimo. Nello scorso anno 1881 infine, il Municipio della Ferrara, sotto la spinta di quell'egregio Sindaco signor Giacomo Stefanini, e coll'aiuto della Provincia e del Comizio Agrario di Verona, ha fatto in Noveza una prima piantagione di 700 Abeti rossi, dei quali oggi, forse per colpa della estate scorsa, soverchiamente calda ed asciutta, solo 400 sono in vita.

Nel Veronese sono coltivate, quali piante di ornamento, non poche specie appartenenti al genere Pinus, le quali sembrano adattarsi molto bene alle condizioni del suolo e del clima: mi limito a citare il $P$. $h a-$ lepensis Mill., ed il P. Pinea L., quest'ultimo conosciuto nel dialetto col nome di Pignolar, sino da epoca assai remota. 


\section{Tribus 2. - Cupressineae.}

Gen. II. - Juniperus.

Sectio. 1. - Oxycedrus.

56. Juniperus communis L. sp. pl. 1470; Pollin. viag. p. 86; fl. veron. III, p. 90, var. a, et herb.!; Bertol. fl. it. X, p. зяs, excl. syn. Juniperi non resiniferi Calc.?; Fr. Font. cat. ctc. p. 30; Ambros. fl. Tir. austr. II, p. 24, var. $\alpha$; Parlat. fl. it. IV, var. a et excl. syn. Calc.?; Vis. et Sacc. cat. p. 65; Nomi volg. etc. p. 73 ; Goir. ert. forest. veron p. 50; Juniperus Calc. viag. di MI. B. p. 12; Ginepro minore ovvero volgare Pona $M$. B. p. 152; Juniperus vulgaris fruticosa Segu.cat.p. 58, et.pl. veron.p. 260; Moren.herb.! - Vernacolo: Ginevro, Zinevro, Zineoro, Zinevrella!, Zinevrelle!, e nel dialetto Cimbro Kranabit. - Icon.: Richb.ic.fl.germ.ethelv.f. 1141. $\alpha$ fruticosa. - - Frutex, plerumque caespitem efficiens; a ramis, ramulisque erecto patulis.

$\beta$ arborea Rchl. fl. germ. exc. I, p. 161. - a Arbor vel " arbusculus: ramis inferioribus, ramulisque pendulis.

Raro nella pianura: comunissimo in tutti i colli ed in tutti i monti della Provincia, nei boschi cedui come nei pascoli aridi e secchi. È per lo più un frutice alto da mezzo metro, o meno, ad un metro ovvero ad un metro e mezzo (var. $\alpha$ ): qualche rara volta, se isolato, nè tormentato dagli animali o dall'uomo, si incontra qua e là con le dimensioni di un vero albero o per lo meno di un alberello che raggiunge l'altezza di 3-4 metri, e anche di più, come ho osservato nel M. Baldo, lungo la via che da Passone conduce a Spiazzi!, nel M. Bolca!, e specialmente nei Lessini in Val di Porro presso la Contrada Capuzzo!, e nel Pastello!(var. $\beta$ ) La qual cosa era già stata notata da Seguier pl. veron. II, p. 260 . a Interdum in justae magnitudinis arborem adolescit, ita ut vix ab ea separandam esse puto "Juniperum vulgarem celsiorem et arborescentem Clus. hist. 38. \- Nelle zone alpina e subalpina, ed anche nella parte più elevata della regione montana, il Ginepro comune è sostituito dal seguente, al quale si passa gradatamente per forme intermediarie. Fiorisce da febbraio e marzo a maggio secondo l'altezza della stazione, o le condizioni climateriche : matura le piccole bacche nell'autunno, ovvero nella primavera dell' anno seguente!. 5 - Presso di noi il Ginepro comune è appena adoperato come combustibile nei monti e nei colli; si fa commercio delle bacche. Per antichissima costumanza nei giorni che precedono la Epifania, i contadini e gli alpigiani scenảono in città a smeriare per le vie e per le case fasci di rami di Ginepro. 
Il Bertoloni ed il Parlatore considerano la pianta, che il Calceolari nel suo viaggio denomina Juniperi non resinosi, quale sinonimo del Juniperus communis. Io ho qualche dubbio a tale proposito: perchè il Calceolari cita questa pianta all' Artillon di M. Baldo, quindi in una stazione alpina: la quale ni sembra più adatta pel Ginepro nano che non al Ginepro comune; per tale considerazione non sono alieno dal ritenere che la pianta del Calceolari sia piuttosto da riferirsi alla specie seguente, a meno che sotto la denominazione di Juniperi non resinosi non si trovino compendiati tanto il $J$. communis quanto il $J$. alpina. Nella zona montana elevata è facile trovare alcune forme che si dimostrano prossime assai al J. hemisphaerica Presl, intermedia, come notò il Parlatore, tra il $J$. communis e il $J$. alpina.

Nei colli e nei monti del Nizzardo ho trovato che il J. Oxycedrus $L$. si presenta benanco, al pari del communis, ora come frutice, altra volta con le dimensioni di un vero albero.

57. Juniperous alpina Clus. hist. I, p. 38; Gren. et Godr. fl. de Fr. IIi, p. 157 ; J. nana W. sp. pl. IV,p. 85; Pollin. viag. p. 99 ; Bertol. fl. it. X, p. 380 ; Reichb. fl. germ. exc. I, p. 161; Vis. et Sacc. cat. p. 65 ; Nomi volg. etc. p. 76 ; Goir. erb. forest. veron. p. 51; J. communis var. nana Pollin. fl. veron. III, p. 191, et herb.!; Ambr. fl. Tir. austr. II, p. 21; J. communis var. alpina Parlat. IV, p. 24; Juniperi non resinosi Calc. viag. di IIT. B. p. 12?; Ginepro di spezie minima Pona M. B. p. 1ro; Juniperus minor montana folio latiore fructuque longiore Segu. cat. p. 58; pl. veron. II, p. 261; Moren. herb.!. - Vernacolo: Zinevro!, Zinevrelle!. - Icon.: Rchb. ic. fl. germ. et helv. f. 1142.

Frutice ramosissimo più 0 meno sdraiato al suolo, che cresce copiosamente dalla parte superiore della regione montana (1000-1200 m.), ove si fonde colla precedente, come già notò il Pollini, sino a tutta la zona subalpina ed alpina. In tutta la catena del M. Baldo, tanto sul versante occidentale che sull' orientale!, nei Lessini!, nel M. Posta!, Campobrun!, Zeola! Alba etc., frequentemente si trova assieme al Pinus Mugus, Rhododendron ferrugineum, Rhododendron hirsutum, Genista radiata ed a molte altre specie suffruticose ovvero erbacee proprie della nostra zona alpina. - Fiorisce in maggio e giugno, e matura i frutti nelle stesse epoche del precedente. 5 - In alcuni punti del M. Baldo, stante la distruzione quasi completa dei boschi, per procurare il calore necessario alle operazioni del caseificio, si è oramai ridotti al Ginepro nano, come unico combustibile, e lo si estirpa senza discrezione e misura alcuna unitamente alle poche ceppaie che rimangono del Fagus sylvatica. 
Il $J$. alpina si distingue a primo aspetto dal $J$. communis, a meno non si tratti di alcuna di quelle forme intermediarie che segnano il passaggio dall' una all'altra specie: nel secondo le foglie sono più lunghe, e più acuminate, sparse, di un verde pallido sulla pagina superiore, intensamente glauche nella inferiore, mentre quelle del primo souo più corte e meno acute, maggiormente appressite le une alle altre, ed offrono un verde caratteristico più o meno cupo; di più nel $J$. alpina $\mathrm{i}$ rami ed i rametti sono più rigidi e tozzi, quantunque i primi frequentemente sieno sdraiati sul suolo.

\section{Sectio 2. - Sabina.}

58. * Juniperus Sabina L. sp. pl. 1472; Pollin. fl. ver. III, p. 192, et herb.!; Vis. et Sacc. cat. p. 65; Nomi volg. etc. p. 77 ; Goir. erb. forest. veron. p. 52. - Vernacolo: Sabina, Erba Sabina.

Nel Veronese è coltivato nei giardini assieme a diverse specie esotiche del genere Juniperus Nel Catalogo delle piante vascolari del Veneto p. $65, n .697$, i signori Visiani e Saccardo indicano questa pianta nei boschi alpini del veronese, ma sino ad oggi io non la ho mai incontrata: il Pollini la descrive nella sua classica Flora, ma la raccolse nel Trentino in Val Sugana herb.!, e ne mandò esemplari al Bertoloni. Ho visto il J. Sabina quasi inselvatichito nei nostri colli, nelle siepi che servono di riparo alle uccellaie.

\section{Gen. III. - Cupressus.}

59. Cupressus sempervirens $L$. sp. $p l$. 1422; Pollin. fl. veron. III, p. 141, var. a; Vis. et Sacc. cat. p. 65; C. pyramidalis Targ. Tozz. obs. bot. dec. 3-5, p. 53. - Vernacolo: Pin, Pigno, Cipresso maschio, Zipresso, Arzipresso, Zipresso mascio.

Coltivato come pianta ornamentale dal piano ai monti. Fiorisce da gennaio a marzo. In Verona destano meritamente attenzione e meraviglia i magnifici cipressi i quali da epoca immemorabile vivono nel giardino del conte Giulio Giusti.

60. Cupressus horizontalis Targ. Tozz. obs. bot. dec. 3-5, p. 54; Vis. et Sacc. cat. p. 65; C. sempervirens Pollin. $f$. veron. $1 I I, p$. 141, var. $\beta$. - Vernacolo: Pigna, Pigno femina, Zipressa, Zipresso femina.

Colla specie precedente. 
Gen. IV. - THuJa.

61. Thuja occidentalis L. sp. pl.; Pollin. fl. veron. III, p. 142 ; Vis. et Sacc. cat. p. 65; Goir. erb. forest. veron. p. 31. - Vernacolo: Tuja, Zipressina del Brasil (Monti).

Adoperata come pianta di ornamento per far siepi. Nei colli veronesi frequentemente con il Juniperus Sabina, si incontra inselvatichita nei pressi delle uccellaie : così p. e. recentemente la ho osservata al Maso! (m. 461). - Fiorisce in febbraio e marzo.

Nei giardini veronesi si coltiva buon numero delle specie più pregiate appartenenti al gruppo delle Strobiliflore. Il sig. Giuseppe Menegazzoli, orticultore tanto intelligente quanto appassionato, mantiene nei suoi vivai una ricchissima raccolta di queste elegantissime piante.

\section{Classis II.}

\section{Anthospermae.}

La classe delle Anthospermae abbraccia i due ordini delle Viscaceae e delle Loranthaceae. Non consta in modo certo, almeno sino a questo giorno, che alcuna delle specie appartenenti a questa classe sia stata raccolta nella Provincia veronese; ritengo però che le esplorazioni future, con ogni probabilità, metteranno gli erborizzatori in grado da affermare la presenza delle Viscaceae nella nostra flora.

Ordo VII.

Viscaceae.

Gen. I. - Viscum.

62. Viscum album L. $s p . p l .1451 ;$ Pollin. fl. veron. III, p. 175 (pro parte?); Viscum baccis albis C. B. pin. p. 423; Moren. herb.

Nè Calceolari, nè Pona, nè Seguier fanno cenno o menzione del Visco nei loro scritti. Il trovarsi questa pianta nell'Erbario del Moreni poco conclude: perchè in via ordinaria questo botanico non indica mai la stazione delle specie da lui riunite, e provenienti in gran parte da località non veronesi. Del Viscum album si conserva un ramoscello nell'Er- 
bario di Fra Fortunato da Rovigo con le denominazioni di Viscum arboreum quercinum, Vischio quercino, 'Visco quercino o vero Pania di quercia, Visco de'Pomi o vero Pania de'Pomari, Visco di Ciriegie o vero Pania de Cerase!: ma non è indicato il luogo in cui è stato raccolto l'esemplare: del resto il paziente compilatore o preparatore dell'Erbario intitolato Theatrum plantarum, non cita questa pianta nell'elenco dello specie da lui osservate o raccolte nel M. Baldo; il quale elenco trovasi inserito a parte nell'ultimo dei sette volumi che formano detto Erbario. Inoltre Fra Fortunato, al pari di altri antichi botanici, confuse molto probabilmente il Viscum album con il Loranthus europaeus: cio sembrerebbero indicare le prime tre frasi sopra riferite, che accennano al secondo, mentre le altre due spetterebbero piuttosto al primo. Ciro Pollini fl. veron. III, p. 175 numera non poche piante arboree o frutescenti sulle quali vive parassita questa singolarissima specie: potrebbe pertanto sembrare, a prima vista, che la stessa almeno ai suoi tempi fosse assai frequente. Ma il ch. Autore nella sua Flora non cita alcun lucgo del Veronese nel quale sia stata da lui o raccolta ovvero osservata: di più nel Catechismo Agrurio ed. I, $p .334$ in nota confessa di non averla mai incontrata nella Provincia. Noto che in questa opera il Pollini confonde manifestamente il Viscum album con il Lorunthus curopaeus, come risulta dal seguente brano l. c.; " somministrano la pania i frutti e la « scorza del visco quercino (viscum album) arbusto parassita di molti alberi «d'Italia ma che non ho giammai rinvenuto nella nostra Provincia:» il quale errore il chiarissimo autore corresse più tardi nella Flora, distinguendo chiaramente nelle note, vol. III, p. 176, il Loranthus europaeus dal Viscum album. Anche il Monti nel suo Dizionario botanico veronese non nomina il Viscum album; nè ai giorni nostri lo rinvennero il Fontana, il Tonini, il Manganotti, il Rigo od altri che io mi sappia; si conserva nelle reliquie fanerogamiche del Prof. Abramo Massalongo, ma raccolto da questi a Possagno in quel di Vicenza nel 1855. Io non sono stato più fortunato: malgrado tutte le più diligenti ricerche sino ad oggi non mi sono mai imbattuto in questa pianta. La quale, del resto, essendo stata rinvenuta nelle zone finitime del Vicentino, del Bresciano e del Trentino, con ogni probabilitì deve crescere, certamente rarissima, benanco presso di noi. Così pure non sono alieno dal ritenere che nelle nostre alpi possa rinvenirsi il Viscum laxum Boiss. et Reut., dai signori Saccardo e Canestrini trovato copioso in Val di Non nel Trentino sul Pinus sylvestris. E siccome il Pollini fra le piante sulle quali asserisce crescere il Viscum album cita pure il Pinus sylvestris, così per conchiudere non posso a meno di esternare il sospetto, che nella pianta descritta da lui, non si trovino riunite e quella antica di Linneo e quella ai giorni nostri descritta dai signori Boissier e Reuter. Tale sospetto mi nasec benanco pel Viscum album dei signori Grenier e Godron $f$. de Fr. II, p. 4, che dal primo di questi due botanici è indicato nella valle del Quayras sul Pinus sylvestris. - Nell' Erbario Polliniano non ho trovato alcun esemplare di Viscum album. - Marzo-aprile. 5 


\section{Ordo VIII.}

\section{LORANTHACEAE.}

\section{Genus 1. - Loranthus.}

63. * Loranthus europaens $L . s p$. pl. 1672; Pollin. fl. veron. III. p. 176 in adnot. ad Viscum album; Nomi volgari etc. p. 85 .

Faccio cenno in questo luogo del Loranthus europaeus, perchè nella opera, già citata, e pubblicata per cura del R. Ministero di Agricoltura, Industria e Commercio, col titolo Nomi volgari adoperati in Italia a designare le principali piante di bosco, è detto che lo stesso nella Provincia di Verona viene chiamato Visco. Da questa indicazione potrebbe nascere la illazione che il Loranthus europaeus sia una specie la quale cresce nella nostra zona botanica. Dichiaro che nelle numerosissime mie erborizzazioni non la ho mai incontrata, come per quanto mi consta, non è stata raccolta da alcuno dei tanti botanici che hanno visitato il Veronese. Siccome anticamente il Loranthus europaeus era adoperato in farmacia, e correva in commercio sotto il nome notissimo di Visco quercino, potrebbe darsi che questa circostanza e questa denominazione abbiano dato origine ad un qualche equivoco, in forza del quale questa pianta si trova indicata nel catalogo ministeriale con nome veronese: nome che a dire il vero, ha presso di noi tutt'altro significato, venendo esclusivamente adoperato ad indicare la pania per la uccellagione, la quale si prepara non gì̀ con le bacche del Loranthus europaens, come è costume in altri luoghi d’Italia, ma bensì con la corteccia della radice del Viburnum Lantana. Nel dizionario della lingua cimbra pubblicato da Marco Pezzo prete Veronese (1763) trovo il vischio indicato col vocabolo laim: osservo che le parole vischio e laim non hanno in questo luogo alcun significato botanico, significano pania e null' altro. 

Al Lom "Mrares the Betta Rienis si

a. Govion

Goiran,

Prodromus Femae Veronemois.

4 parti. 




Estratto dal Nuovo Giornale Botanico Italiano, Vol. XV, N. 1, Gennaio 1883.

PRODROMUS FLORAE VERONENSIS, AUCTORE A. GOIRAN (Continuatio).

Classis III.

Angiospermae.

Subclassis I. - Monocotyledones.

Gli scavi fatti, a scopo di ricerche paleontologiche, nel M. Vegroni di Bolca, dimostrano che sul finire del primo periodo della epoca terziaria, le isole e le sponde continentali bagnate dal mare Eocenico accoglievano una flora splendidissima, affine a quella che oggidì è caratteristica delle regioni intratropicali, tanto del vecchio che del nuovo continente. Destano a buon diritto la meraviglia, di quelli benanco che sono profani alla scienza, i magnifici esemplari appartenenti specialmente ai generi Phoenicites, Latanites, Flabellaria che si conservano nel Civico Museo di Verona. Ma da quell'epoca, che dista dalla attuale forse migliaia e migliaia di secoli, tutto è radicalmente mutato; tanto le condizioni esterne, le igrometriche specialmente e le termiche, 
quanto quelle interne del suolo, i fattori cioè e gli elementi immediati e più essenziali della vita vegetale, in tutte le sue forme e manifestazioni. Il lavorio pertanto lentissimo mia incessante di evoluzione, che tormenta e trasforma la terra, ha gradatamente determinato la successione di nuove specie alle primigenie: però se guardiamo anche soltanto alla maestà ed allo splendore delle forme, l'attuale Flora monocotiledonea neanco può offerire una pallida immagine di quella che nel citato periodo geologico abbelliva la zona che doveva diventare col tempo la Provincia Veronese. Succedanei a quelle stirpi gigantesche non esistono presso di noi: la stessa Palma di S. Pietro martire, che pure vegeta nei colli e nelle rupi marittime della costa occidentale d'Italia ed in quasi tutte le nostre isole, presso di noi si coltiva in vasi che durante la stagione invernale, a meno di anni eccezionali, è uopo internare nelle serre. Tutte le Monocotiledoni veronesi sono allo stato erbaceo; pochissime diventano frutescenti come Asparagus acutifolius e Ruscus aculeatus fra le Asparagaceae, ed Erianthus Ravennae fra le Poaceae; la Arundo Donax, a rigor di termini, nella 'regione nostra appena può dirsi subspontanea. L'Agave americana da epoca già remota inselvatichita sulle sponde del Benaco, rappresenta la specie più gigantesca di questa sotto classe nella attuale Flora veronese.

Ad ogni modo però, nelle Monocotiledoni veronesi sono rappresentati tutti, si può dire, gli ordini che nella penisola e nelle isole italiane costituiscono questo gruppo di Fanerogame; essendo appena eccettuati quelli delle Cannaceae e delle Phoenicaceae. Sono naturalmente predominanti le Orchidaceae, Liliaceae, Juncaceae, Poaceae, Cyperaceae: ma in generale la natura ed il numero assoluto, tanto dei generi, quanto delle specie che figurano nei vari ordini, la densità o frequenza o grado di diffusione delle seconde, il modo e la legge della loro distribuzione sul suolo della Provincia, sono evidentemente dipendenti dalla latitudine, dalla altitudine delle singole stazioni, ed in modo specialissimo dalle condizioni e dalle circostanze locali: la qual cosa, del resto, meglio sarà dichiarata nel corso del presente lavoro. 
Ordo IX.

ORCHIDACEAE.

Orchidee Parlat. fl. it. III, p. 333, excl. gen. Cypripedium.

Nella Flora veronese abbiamo quasi tutti i generi italiani dell' ordine delle Orchidaceae. Ammettendo, che si conservi tuttora presso di noi il genere Aceras ( $A$. antropophora $R$. $B r$.), mancherebbero soltanto i generi Microstylis (MT. monophylla Lindl. = Malaxis monophylla Swartz), Liparis (L. Loeselii C. L. Rich. = Sturmia Loeselii Reichb.), Epipogon (E. aphyllus Aschrs.), Gennaria (G. diphylla Parl.), Barlia (B. longibracteata Parl.), Tinaea (T. cylindracea Biv. = Orchis atlantica $W$.), rappresentati ognuno da un' unica specie. La distribuzione geografica delle ultime tre specie in Italia ed in Europa dà immediatamente la ragione della loro assenza nella Provincia nostra e nelle finitime; la mancanza delle altre potrebbe derivare dall'essere le stesse rarissime. Però la Microstylis monophylla è stata dal Facchini trovata presso Forno in Val di Fiemme nel Tirolo meridionale; la Liparis Loeselii dallo stesso Facchini a Pauls e altrove, e dal sacerdote Paterno al confine del Vicentino col Trentino presso le Tezze; l'Epipogon aphyllus infine, vive nei boschi di faggi e di abeti delle regioni elevate delle Alpi: non sarebbe pertanto impossibile che queste specie insigni, le due prime più particolarmente, venissero un qualche giorno scoperte presso di noi da un paziente e fortunato erborizzatore.

Se ora nella vasta zona che abbraccia il Tirolo meridionale, il Veneto, il Mantovano ed il Bresciano, guardiamo alla quantità numerica delle specie riconosciute sino a questo giorno come viventi nelle singole provincie, troviamo che le stesse sono topograficamente distribuite nelle seguenti proporzioni: ${ }^{1}$

1 Ho ricavato questi numeri dalle seguenti opere: Catalogo delle piante vascolari del Veneto compilato da R. De Visiani e P. Saccardo; Prospetto delle piante vascolari spontanee o comunemente coltivate nella provincia di Brescia per Elia Zersi; Fr. Ambrosii Flora Tiroliae australis; La Provincia di Udine sotto l'aspetto storico naturale, cenni di G. A. Pirona; G. Bizzozero, Seconda aggiunta alla Flora Veneta. 


\begin{tabular}{|c|c|c|c|c|}
\hline Rovigo & ume & $d i$ & spe & 15 \\
\hline Venezia & , & , & " & 25 \\
\hline Mantova & , & " & n & 29 \\
\hline Belluno & D & , & D & 36 \\
\hline Treviso & - & $"$ & , & 39 \\
\hline Padova & , & , & , & 39 \\
\hline Udine & " & " & , & 44 \\
\hline Vicenza & 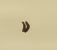 & " & $\Rightarrow$ & 46 \\
\hline Tirolo $\mathrm{m}$ & ridi & ale & 》 & 48 \\
\hline Brescia & - & D & " & 50 \\
\hline Verona & , & , & " & 52 \\
\hline
\end{tabular}

Questi numeri non possono essere che approssimativi; e per la probabile scoperta di forme non ancora osservate, sono per certo soggetti a delle variazioni: le quali però non potranno influire in modo sensibile sulle conseguenze, che legittimamente si possono dagli stessi dedurre. Se poi prendiamo a considerarli assolutamente, devono di necessità dipendere da cause e circostanze molteplici, fra le quali non ultima, il maggior o minor numero di stazioni botaniche esistenti nelle singole provincie, e di abitazioni atte ad accogliere le varie specie dell'ordine. Senza entrare in discussioni, che qui sarebbero fuori luogo, risulta evidente che la provincia di Verona, nella zona considerata, è quella che vanta il maggior numero di specie. Le Orchidaceae italiane essendo rappresentate numericamente da meno di cento forme specifiche, ne segue che il Veronese ricetta più della metà della somma totale delle specie viventi in Italia. La tribù delle Ophrydeae è, come di ragione, predominante; fra tutti poi, il genere Orchis dà il maggior numero di rappresentanti : però la metà appena delle specie italiane appartenenti al genere stesso. Infatti per ragione di latitudine o per altre cause, fra le quali non sono da trascurarsi nè la difficoltà di disseminazione in queste piante, nè probabilmente l'andamento ed il valore delle isotermiche e più specialmente delle isoteriche e delle isochimeniche le quali solcano la parte dell'Italia continentale che dalle Alpi si estende al mare, sono escluse tutte o quasi le specie proprie o caratteristiche della costa occidentale, delle parti centrali e meridionali della penisola e delle isole nostre: taluna di queste però si spinge sino a 
noi, ma o si incontra rara come la Orchis rubra Jacq., ovvero rarissima come la Orchis provincialis. Per lo stesso motivo noi troviamo assai scarso il numero delle specie aprortenenti al genere Ophrys : 5 appena, e taluna assai rara, su 18 che vivono in Italia; e più scarsamente ancura è rappresentato il genere Serapias, ospitando il nostro suolo 2 appena delle 8 specie italiane, Serapias longipetala Pollin. e Serapias Lingua $L$., delle quali inoltre la seconda è rarissima. In quella vece noi possediamo quasi tutte le specie proprie delle regioni alpina, subalpina e montana elevata; e non è improbabile che quelle mancanti, per le future ricerche, possano esse pure rinvenirsi presso di noi.

Le Orchidaceae nostre vivono diffuse sull' intera superficie della Provincia: noto che la sola catena del M. Baldo, cioè la massa montaguosa che sta fra la Valle dell'Adige ed il bacino del Benaco, le alberga quasi tutte; essendone appena escluse pochissime specie. Assai raramente si incontrano nei luoghi coltivati: a seconda della natura e dell'indole delle varie forme specifiche prediligono i luoghi erbosi, i pascoli tanto secchi che umidi o acquitrinosi, i prati naturali, il margine dei campi, gli argini, i cespugli, le macchie, le boscaglie, i luoghi rupestri, le esposizioni ora apriche e soleggiate, altra volta riparate dalle radiazioni solari ecc. Alcune specie crescono solitarie, o per lo meno distintamente separate le une dalle altre come Ophrys muscifera, Ophrys Arachnites, Cephalanthera pallens, Orchis Spitzelii ecc., e non di rado accade di vederle sparire d'un tratto dalla stazione nella quale primieramente avevano sede; altre come Herminium monorchis, Epipactis atrorubens, Cephalanthera ensifolia ecc. sono gregarie e per una serie indefinita di anni si mantengono fisse nello stesso luogo od appena accennano ad un lieve movimento di irradiazione all' intorno; ovvero come Orchis ustulata, Orchis variegata, Orchis militaris, Orchis fragrans, Orchis laxiflora, Serapias longipetala, sparse o adunate in gruppi più o meno numerosi, vivono promiscuamente ed in società, offrendo per tal modo condizioni propizie per la procreazione di non poche forme ibride. Si hanno specie frequentissime e volgari come Orchis Morio, Ophrys aranifera ecc., ed altre rare o rarissime o per lo meno cir- 
coscritte in zone limitate e situate talvolta a grandissima distanza le une dalle altre, p. e. Orchis Spitzelii, Listera cordata, Cephalanthera rubra, Orchis pallens ecc. Alcune specie vivono confinate nelle stazioni della pianura, o nella regione dei colli, ovvero nella zona inferiore della montana, p. e. Spiranthes aestivalis, Spiranthes autumnalis, Ophrys muscifera ecc.; taluna invece non abbandona mai le rupi più elevate delle giogaie alpine, p. e. Chamaeorchis alpina, ovvero non si avanza mai oltre la zona alpina e subalpina come Nigritella angustifolia: e fra questi limiti estremi non poche specie scendono dai pascoli alpini sino alla pianura, nella quale pongono sede duratura, come Listera ovata, Gymnadenia odoratissima, Gymnadenia conopea ecc., mentre altre, p. e. Anacamptis pyramidalis, Orchis variegata ecc. dal piano si spingono in quella vece, o per lo meno lottano per sollevarsi verso le zone elevate dei monti. La Neotiia Nidus-avis e la Corallorhiza innata infine sono Orchidacee humicolae, vivono cioè nel terriccio dei boschi, di faggi, abeti ecc.

Nella nostra zona botanica la fioritura delle Orchidacee. principia in marzo e dura sino alla fine di ottobre, dipendentemente dalla natura delle varie specie e dalla altitudine delle stazioni. Nella pianura fioriscono sin dal marzo la Ophrys aranifera e la Orchis Morio, in settembre ed ottobre la Spiranthes autumnalis.

\section{Tribus 1. - Arethuseae.}

\section{Gen. I. - Limodorum.}

64. Limodorum abortivum Swartz in nov. act. Holm. VI, p. 80 ; Pollin. viag. p. 109, 127 et fl. veron. III, p. 22 (excl. syn. Calc.?) et herb.!; Fr. Font. cat. p. 31; Bertol. fl. it. IX, p. 631; Ambros. fl. Tir. austr. 1, p. 721; Parlat. fl. it. III, p. 344; Vis. et Sacc. cat. p. 58; Orchis abortiva L. sp. pl. 1336; Epipactis abortiva All. fl. ped. II, p. 151; Limodorum austriacum Segu. cat. p. 65 et pl. veron. II, p. 137, excl. syn. Calc.?; Moren. herb. - Icon.: J. B. Barla iconographie des Orchidées tab. 1, fig. 1-21.

Raro nella pianura: da questa sale a tutta la regione collina e penetra nella zona montana, nella quale si eleva qualche volta oltre ai 1200 
metri di altitudine. Si incontra qua e là, ora isolato, altra volta in gruppi più 0 meno numerosi: però non si può dire comnne. Io lo ho osservato specialmente nei boschi di querce; è meno frequente in quelli di castagno; rarissimo nei boschi di faggio; lo ho pure visto crescere lussureggiante frammezzo ai Fraxinus Ornus, Carpinus Betulus, Ostrya carpinifolia, Ulmus campestris, Corylus Avellana, Viburnum Lantana, Cytisus sessilifolius, Juniperus communis ecc.: però è per lo meno dubbio il suo parassitismo, da taluno asserito, sulle radici delle diverse specie frammezzo alle quali si ritrova. - Nella zona e nei colli Benacesi a Lazise (Fr. Fontana, Rigo), alla Rocca di Garda (Rigo), Scaveaghe!, Bré!, Torri (Rigo) ecc.; alle falde orientali del M. Buldo lungo la salita da Brentino alla Corona!, stazione pure indicata dal Pollini, il quale inoltre nel suo viaggio ( $p .109)$ lo segnala alle falde settentrionali dello stesso monte verso Tierno, Castione, Brentonico ecc., mentre io lo ho incontrato lungo la Aviana nella ascesa da Avio al pian della Cenere!, e nel corpo istesso della catena, isolato, e non tutti gli anni, in Basiana!, Ime!, alla Lonza!; nel MI. Fastello!; nella valle dell'Adige p. e. a Rivole, lungo la salita da Peri alle Fosse! ecc.; nell' Agro veronese presso Guastalla! (e Pollin.herb.!); ad Ospedaletto (Pollin.herb!); nel bosco Mantico!; sopra il Maso nel Roccolo!; nella val Pantena presso Quinto!, Poiano!, Grezzana! (e Tonini) ecc. ; nei colli di Montorio!, Marcellise!, ed alla Mosella! ove è pure stato rinvenuto dal Seguier; nel $M$. Trezzolan!; nella valle d' Illasi presso Tregnago! (e da Campo herb.!, A. Mass. herb.l) etc. L'amico Abate Francesco Masè to ha raccolto nel Mantovano. - $\mathrm{E}$ in fiore da aprile a luglio secondo la altitudine, le condizioni locali e l'audamento della stagione. 20

Come è noto, in questa specie la forma del labello è assai variabile: nelle piante veronesi, talora sopra uno stesso individuo, mi fu dato esaminare fiori che presentavano nel labello le varie forme accennate dai fitografi. Nel $\boldsymbol{M}$. Pastello segnatamente, ho osservato la forma col labello quasi tondo, corrispondente al Jimodorum sphaerocephalum del Viviani. L'altezza del fusto di questa Orchidacea è non di rado assai considerevole: ho misurato individui i quali superavano l'altezza di un metro!.

Il Seguier, e dopo di lui Ciro Pollini hanno considerato la Orobanche indicata dal Calceolari, a p. 12 del suo viaggio, nella valle dell' Artillon, quale sinonimo del Limodorum abortivum: ma io credo sia assai difficile lo affermare con sicnrezza, quale pianta sotto la denominazione di Orobanche siasi voluta significare dal Calceolari, essendo la stessa troppo vaga ed incerta e mi sembra più naturale il ritenere che il Calceolari abbia inteso accennare effettivamente ad una Orobanche, taluna specie di questo genere essendo volgatissima nel M. Baldo. Tenendo conto della altezza considerevole, che come ora si è detto è raggiunta qualche volta dal fusto del nostro $L_{i-}$ modorum, con egual criterio sarebbe forse da riferirsi a questo l'Orobanche molto grande dello stesso autore (viag. p. 13): ma la stazione assegnata a questa pianta, cioè Monte maggiore, ed il trovarla elencata fra specie prettamente alpine, mi fanno rinunziare a questa supposizione. Del resto si tratta di semplici congetture, che cadono naturalmente, se in 
realtà l'Orobanche citata a pag. 12 dal Calceolari corrisponde al Limodorum abortivum: $\mathrm{ma}$ in tal caso questa Orchidacea si troverebbe pure compresa nella Orobanche di varie forme che il Pona, M. B. p. 168 dice crescere in Basiana ed in Ime.

\section{Gen. II. - Cephalanthera.}

65. Cephalanthera ensifolia C. L. Rich. in mém. du Muséum IV, p. 60; Bertol. fl. it. IX, p. 62s; Ambros. fl. Tir. austr. I, p. 721; Parlat. fl. it. III, p. 347; Vis. et Sacc. cat. p. 58; Goir. app. bot. in cron. alp. p. 78; Epipactis grandiflora All. fl. ped. II, p. 152; Epipactis ensifolia Pollin. fl. veron. III, p. 35 et herb.! - Icon.: Barla op. cit. tab. 2, fig. 1-12.

- Species variat magnitudine florum, longitudine folio" rum et bractearum. Rachis saepius laevis; quandoque, - sub vitro, asperitatibus minutissimis adspersa; ovaria - nunc glaberrima; alia vice glandulis parvis, sessilibus vel " subpedicellatis praedita. Duae varietates, vel potius formae, - occurrunt.

a brevifolia. - - Spica elongata: foliis supremis spica brevioribus. "

$\beta$ longifolia. - Floribus aliquantulum majoribus: spica " abbreviata: foliis supremis et plerumque bracteis infimis a spicam aequantibus superantibusve.

Questa bella specie si incontra qua e là, solitaria ovvero in gruppi; di preferenza nei boschi di querce della regione collina, più raramente nella pianura; è copiosissima in talune stazioni, ma non può dirsi, nel complesso, molto frequente o comune. - In sylvis collinis veronensibus una vice mihi se obtulit, nec loci peculiaris memini: così Ciro Pollini op. et loc. cit. p. 36 : ed infaíti si conserva nell' Erbario Polliniano un esemplare di questa pianta, raccolta nei colli veronesi, ma senza indicazione di luogo. - Alla Mosella! (91 m.); presso Montorio! e Marcellise!; nel M. Trezzolan! (412 m.); al Maso nel luogo detto il Roccolo (520 m.); nel M. Pastello sopra Cavalo! (600 m.) e Monte!; a Costermano!; alle sponde del Benaco a Scaveaghe! e S. Vigilio!; e più in alto nei colli Benacesi in Brè!, ove la ho trovata in società con la Cephalanthera pallens, mentre l'amico Gregorio Rigo la segnala copiosissima sopra Torri ed alla Rocca di Garda; nella valle d'Illasi nel $M$. Barbara ove è stata raccolta da Abramo Massalongo!. Probabilmente crescerà ancora in altri punti: l'Arciprete Masè la ha raccolta nella pianura veronese a S. Pietro in Valle.Fiorisce dalla fine di aprile a giugno: le due forme $\alpha$ e $\beta$ si incontrano per lo più unite assieme. is 
Non è esatto che questa Orchidacea prediliga le stazioni ombrose ed umide, come trovo scritto in alcune flore; avendola per lo più osservata in luoghi aridissimi ed esposta a vivissima radiazione solare. La esposizione e la condizione idrologica del suolo, hanno solo influenza per determinare nella piauta una maggiore o minore esuberanza di vegetazione: così ad esempio, dipendentemente da queste condizioni la spiga è più 0 meno fornita di fiori, maggiore o minore l'altezza della pianta, la larghezza delle foglie ecc.

Nella Cephalanthera ensifolia la rachide che porta i fiori, è frequentemente sparsa di minute scabrosità; e lo stesso può dirsi degli ovari: questi generalmente sono glabri, ma non di rado portano delle piccole ghiandole ora sessili, altra volta pedicellate. I fiori variano essi pure di grandezza; nella var. $\beta$ le loro dimensioni sono per lo più superiori a quelle che si notano nella var. $\alpha$. Le brattee inferiori si trasformano frequentemente in lamina fogliacea, la quale nella var. $\beta$ raggiunge tale lunghezza da sorpassare non solo il fiore corrispondente ad essa, ma l' inter s spiga. In alcuni esemplari veronesi, ad esempio, la spiga è lunga 6 centimetri, mentre la brattea spettante all'ultimo fiore è lunga sino a 10 centimetri. Tutte queste particolarità io ho notate non solo nel gran numero di esemplari veronesi che ho potuto esaminare, ma nelle piante benanco provenienti da altre stazioni italiane, p. e. dalle provincie meridionali, e favoritemi dal chiarissimo prof. Pedicino.

Il diligente ed oculatissimo Seguier non accenna a questa pianta, a meno che sotto la denominazione di Helleborine flore albo vel Damasonium montanum latifolium non abbia inteso indicare tanto la Cephalanthera pallens quanto la Cephalanthera ensifolia. Sembrerebbe del resto che la stessa fosse rarissima anche ai tempi di Ciro Pollini, almeno giudicando dalle parole di lui che sopra ho riferite: badando pertanto alla distribuzione ed al grado attuale di frequenza della Cephalanthera ensifolia sul suolo della Provincia, sarebbe da conchiudersi che per questa Orchidacea, siasi da quell'epoca considerevolmente allargata l'area di vegetazione.

66. Cephalanthera pallens $C$. L. Rich. in mém. du Muséum IV, p. 60; Ambros. fl. Tir. austr. I, p. 722; Parlat. fl. it. III, p. 349 ; Vis. et Sacc. cat. p. 58; Epipactis pallens Pollin. fl. veron. III, p. 35; Fr. Font. cat. p. 29; Cephalanthera grandiflora Bab. man. of. brit. bot. p. 296; Bertol. fl. it. IX, p. 626; Helleborine flore albo vel Damasonium montanum, latifolium Segu. cat. pl. p. 53 et pl. veron. II, p. 136; Moren. herb. - Icon.: Barla op. cit. tab. 3, fig. 1-21. $\beta$ uniflora - Planta pumila. Spica uniflora."

Nei luoghi boschivi e selvatici della regione collina e montana, però non comune; più raramente nella pianura e nella zona subalpina. Nel M. Baldo alla Lonza in mezzo ai faggi!; presso Grezzana (Seguier); 
nei M. Lessini a Corbiolo! e presso Chiesanova! (e Pollini); nella Valle d'Illasi presso Tregnago, nel bosco di F'errari e nella Valle dell Orso (A. Mass. herb.!); nella Riviera e nei Colli Benacesi, presso Lazise (Pollini, Fontana), alla Rocca di Garda (Rigo), sopra Torri e S. Vigitio in Brè! (e Rigo); a Costermano!; Ospedaletto (Pollini), Guastalla! (e Pullini); nel M. Pastello ecc.; nella pianura l'Arciprete Masè la ha raccolta a Campalano. La var. $\beta$ nel $M$. Baldo alla Lonza colla forma tipica. Fiorisce nella stessa epoca della precedente, colla quale si trova qualche volta in società. 2

67. Cephalanthera rubra $C$. L. Rich. in mém. du Muséun p. 60; Bertol. fl. it. IX, p. 629; Ambros. fl. Tir. austr. I, p. 725; Parlat. fl. it. III, p. 350; Vis. et Sacc. cat. p. 58; Serapias Helleborine $\delta L . s p . p l$. 1314; Epipactis rubra All. fl. ped. 1I, p. 15.3; Pollin. viag. p. 16, 122 et fl. veron. III, p. 36; Franc. Font. cat. p. 29; Helleborine montana, angustifolia, purpurascens Segu. cat. pl. p. 53 et pl. veron. II, p. 136; Moren. herb. - Icon.: Barla op. cit. tab. 4, fig. 1-18.

\& oligantha - « Spithamea, subtriflora: floribus mi- noribus quam in specie, foliisque angustioribus. 》

Nei luoghi selvatici e boschivi delle regioni collina e montana, dalle quali accerna ad avvicinarsi alla zona subalpina; non la ho mai incontrata nella pianura: è pianta piuttosto rara. - Nella regione del Benaco presso Lazise (Fr. Fontana) e nei boschetti della Rocca di Garda (Rigo); nel M. Baldo in Basiana ed Ime (Pollini), nella Valle dell'Acqua!, nel Pian della cenere (Rigo); presso Novare in Val Policella (Pollini); nei Lessini presso S. Anna d'Alfaedo!, nel vajo del Falcone! ed in quello dell'Anguilla!, presso Lughezzano!, Chiesanova!; nella Val Pantena nei pressi di Grezzana (Seguier); nel Bosco del Romitorio presso il MLaso!; nella Valle d'Illasi presso Tregnago; a Vestena e Castelvero (A. Mass. herb.!) ecc.: nel complesso si trova dunque sparsa sulla zona montagnosa o collina che dal confine vicentino si distende alle sponde del Benaco, sino ad oltre 1200 metri di altitudine. - $\mathrm{E}$ in fioritura dalla fine di maggio al termine di luglio secondo la altitudine e le condizioni della stazione. 2

La mia varietà $\beta$ si distingue dalla specie per essere in tutte le sue parti più piccola e delicata di questa, e per la spiga che è poverissima di fiori: la ho raccolta vel $M$. Baldo nella Valle dell'Acqua. Ho pure incontrato specialmente nei luoghi un po' umidi, una forma con le foglie assai grandi, 11-12 cent. lunghe e 2 cent. larghe; e con la spiga lunga 15 centimetri formata da 12-15 fiori. Il Seguier scriveva di questa pianta che: "Florum spica modo longa et modo brevis cauli insidet. Multum * etiam in foliis ludit » (Pl. veron. $I I, p .13 \%)$. 
Tribus 2. - Neottieae.

Sub-tribus 1. - Listereae.

Gen. III. - EpIPaCtis.

Sectio 1. - Arthrochitium Irmisch, Reichb. fil.

68. Epipactis palustris Crantz stirp. austr. p. 462; Pollin. fl. veron. III, p. 33; Bertol. fl. it. IX, p. 620; Ambros. fl. Tir. austr. I, p. 726; Parlat. fl. it. III, p. 355; Vis. et Sacc. cat. p. 58; Serapias longifolia L. syst. nat. edit. 12, t. II, p. 593 ; Serapias palustris Scop. fl. carn. ed. 2, t. II, p. 201; Epipactis longifolia All. fl. ped. II, p. 152; Helleborine pratensis angustifolia flore herbaceo Moren. herb. - Icon.: Barla op. cit. tab. 5, fig. 1-17.

$\beta$ humilis - a Spica pauciflora. Tota planta vix spithamea.

Rara; nei luoghi paludosi, od umidi e sabbiosi dalla pianura ai monti: presso Legnago! (e Pollini); nei boschi lungo l'Adige al Casino!, Giarone!, Mambrotta!; nella valle dell'Adige presso Rivole, Incanale! ove l' ho sempre incontrata in piccoli gruppi; l'amico Gregorio Rigo la ha rinvenuta nei prati fra Garda e Costermano: probabilmente a parità di condizioni, si troveri in altri punti della Provincia non solo nel piano ma benanco nei monti. La var. $\beta$ cresce qua e là assieme alla forma tipica. - Fiorisce in giugno e luglio, almeno nelle stazioni reronesi nelle quali sino ad oggi ho trovato questa pianta. 2

Ho pure raccolto l' Epipactis palustris nella Naunia (Trentino), in un luogo paludoso presso Cles, lungo la via che conduce alla pittoresca e selvaggia valletta di Tovel.

Sectio 2. - Euepipactis Irmisch, Reichb. fil.

69. Epipactis latifolia All. fl. ped. II, p. 152: Swartz in act. Holm. ann. 1800, p. 232; Pollin. viag. p. 122, 126 et fl. veron. III, p. 34 pro parte (et excl. nonn. syn. ?); Bertol. fl. it. IX, p. 623 pro parte; Ambros. fl. Tir. austr. I, p. 728, var. a; Parlat. fl. it. III, p. 357; Vis. et Sacc. cat. p. 58 ; Serapias Helleborine $L . s p$. pl. 1344, var. a; Serapias la tifolia L. syst. nat. edit. 12, tom. II, p. 193 et mant. alt. p. 490. - Icon.: Barla op. cit. tał. 6, fig. 1-11. 
$\beta$ viridiflora Reichb. fl. germ. exc. p. 131. - Icon.: Barla op. cit. tab. 7 frg. 1-4.

Dalla regione alpina ai colli, internandosi benanco nelle valli ed avvicinandosi alla pianura nella quale compare qua e là sporadicamente: cresce fra le boscaglie ed i cespugli tanto nei luoghi umidi ed ombrosi, quanto nelle starioni maggiormente apriche e soleggiate. È pianta gregaria, che vive in piccole famiglie, dimostrando una tal quale predilezione pei luoghi sassosi : si trova però anche isolata, ma più di raro. Nel M. Baido all'Arlitlon! (e Rigo), al Tret de spin (Rigo), Noveza!, Lonza!, Valfredda! (e De Bracht), Ime!', Basiana!, La Ferrara!, Albaré! ecc.; alla Rocca di Garda (Rigo) e presso Lazise nel bosco Alberghini (Rigo); in tutta la catena dei Lessini e nei monti dipendenti!; nella valle Pantena! (e Tonini!); nel vajo del Falcone!, nel vajo dell'Anguilla!; nella valle d'Illasi!; nei monti confinanti col Vicentino!; a S. Ambrogio!, Ospedaletto!, Guastalla! ecc. L'amico Masè mi scrive di averla raccolta nella pianura a S. Giovanni in Valle. - Ho osservato esemplari appartenenti alla var. $\beta$ (Giugno 1876) in Valpantena presso Costoli. - Fiorisce da maggio a luglio ed agosto dipendentemente dalla altitudine.

Nella Epipactis latifolia tanto del Pollini quanto del Bertoloni sono riunite e la vera $E$. latifolia All. e la $E$. atrorubens Schult. Così pure le piante del Pona Helleborina del Lobelio (M. B. p. 211), Helleborina ovvero Epipattide del Pona, del Lobelio e del Dodoneo (M. B. p. 230), e la Helleborine latifolia montana del Seguier (cat. pl. p. 53, et pl. veron. II, p. 138) abbracciano secondo ogni probabilità tanto la prima quanto la seconda forma.

70. Epipactis atrorubens Schult. oesterr. $f l . I, p .5 S$ ex Lindl.; Parlat. fl. it. 111, p. 359; Vis. et Sacc. cat. p. 58; E. latifolia Pollin. viag. et fl. veron. l. c. pro parte; Bertol. fl. it. IX, p. 623 pro parte; E. latifolia $\beta$ rubiginosa Gaud. fl. helv. $V$, p. 465; E. rubiginosa Koch syn. fl. germ. et helv. edit. 2, p. 801; E. latifolia $\beta$ atrorubens Ambros. $f l$. Tir. austr. I, p. 728. - Icon.: Barla op. cit. tab. 7, fig. 5-12.

Nelle selve e nei boschi montani della Provincia, dai quali scende nei colli e nelle valli procedendo verso la pianura: frequentissimamente nelle stazioni e nei luoghi della precedente colla quale cresce in società. Nel M. Baldo, copiosissima tanto sul versante orientale che sull'occidentale, p. e. nella salita da Brentino alla Corona!, in Basiana!, la Ferrara! ecc.; presso Malcesine!; alla Rocca di Garda (Rigo); nei Lessini presso Lughezzano!, Chiesanuova! ecc. ecc. - $\grave{\mathrm{E}}$ in fioritura nella stessa epoca della precedente. 2

In questa forma la lunghezza delle brattee, le inferiori specialmente, è variabilissima: queste ultime infatti, se frequentemente eguagliano appena l'ovario, altra volta invece, trasformate in lanine fogliacee, sono 
lunghe per modo, da egungliare non solo, ma da superare il fiore. Nelle foglie le nervature ora sono totalmente liscie, altravolta coperte da numerose scabrosità. Varia pure il grado di pubescenza nei tepali esterni e nel peduncolo che porta il fiore. Sono mutabili infine la forma e la grandezza delle foglie e le distanze degli internodi. Frequentemente mi sono imbattuto in individui che segnano un vero passaggio alla specie precedente; ed altravolta p. e. nel M. Pastello nel Veronese, e presso Fondo nella Naunia (Tirolo mexidionale), ho notato delle forme che si mostravano vicinissime alla specie che segue. Osservo ancora che la tinta variabilissima che presentano nei fiori la $E$. latifolia e la $E$. atrorubens, non solo dipende da condizioni locali, come già scrisse il Seguier, ma benanco dalle circostanze esterne. Difatti esaminando, durante un periodo di oltre dieci anni, sempre nelle stesse stazioni, alcuni gruppi di questa Orchidacea, osservai di anno in anno e talvolta nel corso di una stessa stagione, che le tinte ed i colori dei fiori mutavano a seconda àelle condizioni meteoriche.

71. Epipactis microphylla Swartz in act. holm. ann. 1800, p. 232; Bertol. fl. it. IX, p. 622; Parlat. fl. it. III, p. 361; Vis. et Sacc. cat. p. 5s. - Icon.: Barla op. cit. tab. 8, fig. 1-16.

Rarissima. - Una volta soltanto ho raccolto un esemplare di $E$. $m i$ crophylla nel M. Pastello sopra Cavalo in un boschetto di querce: probabilmente crescerà in altri punti della Provincia. L'esemplare raccolto allora da me, deve trovarsi nell' erbario del sempre compianto amico Prof. Ferdinando Rosellini, in Casale Monferrato. - Fiorisce in giugno e luglio. 2

Nell' Erbario Polliniano, sotto la scheda Serapias Helleborine var. foliis angustioribus, ma senza designazione di località, si trova un esemplare che sembra doversi riferire alla $E$. microphylla.

Nel disporre le Orchidacee veronesi, avendo prescelto l'ordine e la distribuzione adottata dal Prof. Filippo Parlatore nella sua Flora Italiana, ho mantenuto come tre specie distinte la E. latifolia, E. atrorubens, $E$. microphylla: le quali però, meglio sarebbero da ritenersi quali semplici varietà, o meglio forme, di un tipo unico; come del resto hanno opinato alcuni illustri fitografi.

\section{Gen. IV. - Neottia.}

72. Neottia Nidus-avis C. L. Rich. in mém. du Muséum $I V$, p. 51; Bertol. fl. it. IX, p. 614; Ambros. fl. Tir. austr. I, p. 730 ; Parlat. fl. it. III, p. 364; Vis. et Sacc. cat. p. 59; Ophrys Nidus-avis L. sp. pl. 1339 et mant. alt. p. 488; Epipactis Nidus-avis All. fl. ped. II, p. 151; Pollin. viag. p. 113 et fl. veron. 1II, p. 36; Satirio abortivo del Lobelio 
Pona M. B.p. 23S; Satyrium abortivum Lobelii Segu. cat. pl. p. 91 et pl. veron. II, p. 357 ex Pona. - Icon.: Barla tab. 9 fig. 1-12.

Vive nel terriccio dei boschi di Faggi, di Abeti, di Mughi!, e probabilmente di altre essenze silvestri o boschive, quali Sambucus racemosa, Rhododendron ferrugineum e hirsutum, Genista radiata ecc. Questa bellissima Orchidacea si incontra ora in piccoli gruppi, altre volte solitaria, ma non molto frequentemente, in tutta la zona subalpina dei nostri monti, dal versante occidentale del $\boldsymbol{H}$. Baldo al confine Vicentino: penetra benanco nella zona alpina ma assai di raro, e scende nella regione montana internandosi nelle valli: compare rarissimamente nei colli a poca altezza sul Benaco. - Il Pona indica il Sativio abortivo del Lobelio nel M. Baldo all'Artillon: Seguier che cita questa pianta sulla fede del Pona, dichiara di non averla mai trovata: Ciro Pollini osservò copiosissima la Neottia Nidus-avis nel M. Baldo nelle selve di faggio che circondano la depressione detta il Piano della Cenere (fl. ver. l. c.), come pure al disopra di questo luogo ai Lavacci (viag. l. c.); e nei $M$. Lessini presso Chiesanuova e le Scandole. Io ho osservato o raccolto questa Orchidacea non solo nelle località indicate dal Pona e dal Pollini ma in molti punti: p. e. nel M. Bolca!; nella valle d'Illasi presso Selva di Progno e S. Andrea! ecc.; nei Lessini a Revolto! (e Caro Massalongo), ai Trachi!, ai Tinazzi!, al passo della Sega! ecc.; nel M. Baldo nella Valfredda!, alla Lonza!, in Novezina e Noveza! ecc. L'amico Gregorio Rigo infine ne ha raccolto il 6 maggio 1873 tre esemplari nei colli Benacesi, sopra Torri presso il luogo chiamato Bré, framezzo alle querce, a meno di $300 \mathrm{~m}$. sul livelio del lago.

Nel Veronese pertanto la Neottia Nidus-avis dalla regione alpina scende a quella dei colli. Osservo che il Sig. Francesco Negri l' ha raccolta nei boschi del Casalese!; ed il Cav. G. B. Barla presso Nizza nella collina di Cimella, a pochissima distanza cioè dalle sponde del mare: quindi è lecito conchiudere che questa Orchidacea dalla regione alpina si spinge, si può dire, sino alle sponde del mediterraneo. Non sussiste pertanto l' affermazione dei Sigg. Grenier e Godron (Fl. de Fr. III, p. 275) che la Neottia Nidus-avis " manque dans la région méditerranéenne et " dans la région des oliviers. »

Dipendentemente dalla altitudine e dalla latitudine della stazione la Neottia Nidus-avis muta di abito, o meglio se si vuole di aspetto: le forme più meridionali si presentano più piccole e più delicate in tutte le loro parti ; così p. e. gli esemplari raccolti nei colli Benacesi dal Rigo, riproducono si può dire esattamente la pianta trovata dal Barla a $C i$ mella nelle vicinanze di Nizza, e da questi riprodotta nella tav. 9, fig. 8 della sua opera intitolata Iconographie des Orchidées. Anche la tinta è assai variabile, essendo ora più, ora meno fosca, e secondo ogni probabilità dipendentemente dalla natura e dalla costituzione dell'humus nel quale la Neottia è astretta a vivere. L'altezza del fusto è variabilissima; 
tengo esemplari veronesi ed altri favoritimi dal Irof.re G. Gibelli e provenienti dall'alto Appennino modenese, lunghi sino ai 5 decimetri, mentre quelli Casalesi del Negri appena toccano i 15 centimetri. Il fusto che ordinariamente è diritto, qualche volta è contorto e sinuoso come negli esemplari da me raccolti alla Lonza di $M$. Baldo.

Fiorisce da maggio a luglio secondo i luoghi. $z_{0}$

Gen. V. - Listera.

73. Listera ovata R. Brown. in Ait. h. kew. V, p. 201; Bertol. fl. it. IX, p. 616; Ambros. fl. Tir. austr. I, p. 732; Parlat. fl. it. III, p. 36r; Vis. et Sacc.cat. p. 58; Ophrys ovata L. sp.pl.1340; Epipactis ovata All. fl.ped.II, p.151; Pollin. viag. p. 116 et fl. veron. III, p. 37; Fr. Font. cat. p. 29; Bisfoglio Calc. viag. di M. B. p. 11; Ophrys Calc. viafl. di 11. B. p. 12?; Orchis falso, o Bifolio del Dodoneo, da alcuni Ofris Pliniano creduto; nè mancano alcuni che lo dissero Grame di Parnasso Pona M. B. p. 189; Ophrys bifolia Segu. cat. pl. p. 76 et pl. veron. II, p. 138; Pollin. herb.! - Icon.: Barla op. cit. tab. 9, fig. 17-26.

Dalla regione subalpina e montana, nelle quali cresce copiosissima, scende ai colli; si interna pure nelle valli e si avanza verso la pianura nella quale compare qua e là: la ho osservata benanco nella regione alpina. Vive nei prati, nei pascoli e nei luoghi boschivi ombrosi. - In tutta la catena del $M I$. Baldo; sul versante orientale in Pravazar!, Ime!, Valfredda!, alla Ferrara!, Noveza! (e Pollini), Artillon (Calceolari), e sull'occidentale in Ortigara!, val Vaccara!, presso il fonte di Brigaldello! ove venne pure indicata dal Pona, ecc.; alle sponde del Benaco presso Torri (Rigo!) e Lazise (Fontana); nella val d'Adige! (e Ambrosi); in tutta la catena dei Lessini!, scendendo dai pascoli elevati nel vajo del Falcone!, nel vajo dell' Anguilla!, nella Val Pantena sin presso Grezzana (Seguier, Tonini), nel rajo di Squaranto, nella valle d'Illasi sin presso Tregnago! (A. Massalongo!); nel M. Bolca! ecc.; si trova presso Verona alle sponde dell'Adige nell' isolotto detto il Pestrino!, stazione indicata pure da Ciro Pollini; nella pianura infine Pollini la raccolse a S. Pietro in Valle (herb.!), io nei prati umidi presso Vigasio!. L'amico Masè mi scrive che questa specie cresce pure nel Mantovano. - $\hat{\mathrm{E}}$ in fiore alla fine di aprile nella pianura; in maggio e gingno nelle stazioni elevate. 2

Secondo Seguier e Pollini, l'Ophrys o Ofride di Calceolari (viag. di MI. B. p. 12) corrisponderebbe alla nostra pianta: per veriti io ho su questo punto un qualche dubbio, e sospetto che sotto tale denominazione il Calceolari abbia voluto indicare una qualche altra Orchidacea. Tale sospetto nasce in me da ciò che il Calcsolari indica la sua Ofvide per la stessa lo. calità nella quale dice di aver osservato il Bisfoglio. Se si trattasse di 
una stessa pianta, mi pare che il Calceolari non avrebbe nello stesso elenco ed a breve distanza, adoperate distinte le denominazioni di Bisfoglio e di Ofride per designarla. Che l' Ofride segnalata dal Calceolari all'Artillon corrisponda al Satirio abortivo (Neottic Nidus-avis) trovata posteriormente dal Pona nella stessa località?

La Listera ovata ha una statura assai variabile; i miei esemplari oscillano tra 45 e 20 centimetri; i fiori che formano la spiga sono ora numerosissimi, altra volta radi e pochi; le foglie largamente ovate o elittiche ed ottuse nella forma tipica, in alcuni esemplari raccolti nel $\boldsymbol{M}$. $B a l d o$, sono invece distintamente lanceolate ed acute. Dal rizoma, indipendentemente dal fusto fiorifero, si dipartono fusti secondari, e sterili in seguito all'abortimento dello scapo destin ato a portare la spiga.

74. Listera cordata $R$. Brown in Ait. h. liew. V, p. 201; Bertol. fl. it. IX, p. 618; Ambros. fl. Tir. austr. 1, p. 733; Parlat. fl. it. III, p. 369 ; Vis. et Sacc. cat. p. 59; Ophrys cordata L. sp. p'. $13 \hat{1} 0$ var. a; Epipactis cordata All. fl. ped. II, p. 152; Pollin. fl. ver. III, p. 39 in notis ad gen. Epipactis. - Icon.: Barla op. cit. tab. 9, fit. 1\%-26.

Rarissima Fu scoperta primieramente dal sig. Francesco Fontana, farmacista a Lxzise, sul $M$. Baldo nel lnogo detto le Busette (2000 m.)!, ove venne pure raccolta da Manganotti e Tonini!: il Prof. Abramo Massalongo la trorò nel 1849 noi $\boldsymbol{M}$. Lessini nei luoghi erbosi e rupestri del Malèrx!. Nell' erbario del Pollini se ne trova un esemplare ma senza indicazions d lla localiti di provenienza. - Fiorisce da giugno a luglio secondo la altitudine. $\tilde{z}$

\section{Subtribus 2. - Spirantheae.}

\section{Gen. VI. - Spiranthes.}

75. Spilanthes aestivalis $C$. L. Rich. in mém. du IVuséum $I V$,

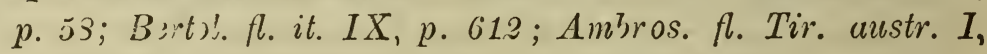
p. 735; P.trut. fl. it. III, p. 372; Vis. et Sacc. cat. p. 59; Ophryz spiralis $\gamma$ L. sp. p'. $13 t 0$; Neottia aestivalis Dec. fl. frxi. III, p. 2j3; Pullin. fl. verun. III, p. 32. - Icon.: Burle op. cit. tr') 10 ,is. 1-5.

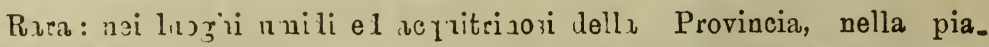
nura specialmente. Il Pollini la indica presso Buolone, inter iuncos radices ajen 3 ; il M $\mathrm{M}$ ๖̀ ed il dott. R) le rhér la scopersero presso Nogàra; l'Amb:Jsi op. cit. p. 73 j scriva c's e cresca fra Riva e Torbcle sul Benaco.

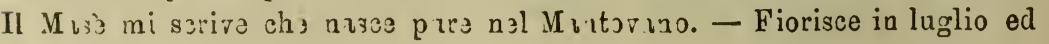
agวงว). 2 
76. Spiranthes autumnalis $C$. $L$. Rich. in mém. du Muséum IV, p. 59; Bertol. fl. it. IX, p. 610; Ambros. fl. Tir. austr. I, p. 736; Parlat. fl. it. III, p. 374; Vis. et Sacc. cat. p. 5?; Ophrys spiralis L. sp. pl. 1310; Epipactis spiralis All. fl. ped. II, p. 152; Neottia spiralis Willd. sp. pl. IV, p. 74 var. $\alpha$; Pollin. fl. veron. III, p. 31; Fr. Fontana cat. p. 31; Orchiastrum autumnale pratense, spirale, album, odoratum Segu. pl. veron. 111 (Suppl.), p. 252; Moren. herb. - Vernacolo: Castagnole. - Icon.: Segu. loc. cit. tab. VIII, fig. 9; Barla op. cit. tab. 10, fig. \%-12.

Nei luoghi erbosi, tanto umidi che secchi, dalla regione collina alla pianura. Il Seguier la indica in collibus ad Benacum vergentibus solo aprico; e nei colli Benacesi la raccolsero pure il Fontana presso Lazise, e Gregorio Rigo nei dintorni di Torri nei prati di S. Antonio, a Forno ecc.; da Pollini e Lorei fu trovata nei pressi di Verona in Campo Marzo, e nei colli di Sommacampagna; da Abramo Massalongo a Bussolengo!; da Antonio Manganotti nel Bosco Mantico; io ho visto copiosissima la Spiranthes autumnalis nei dintorni di Rivole!, ed il dott. Rodeghér recentemente me la ha segnalata a Nogàra nella bassa pianura veronese. Cresce pure nel Mantovano, come mi scrive il Masè. - Fiorisce in settembre ed ottobre. 2

I fiori mandano odore acutissimo, ora di vaniglia, altra volta di mandorla amara: il labello che per lo più è verdognolo, fu riscontrato frequentemente da me perfettamente candido, tanto nel Veronese che nella Liguria.

\section{Gen. VII. - GOODYerA.}

77. Goodyera repens I. Brown in Ait. h. liew. V, p. 198; Bertol. fl. it. IX, p. 60s; Ambros. fl. Tir. austr. I, p. \%34; Parlat. fl. it. III, p. 377; Vis. et Sacc. cat.p. 59; Satyrium repens L. sp. pl. 1339; Epipactis repens All. fl. ped. II, p. 15:; Neottia repens Swartz in act. holm. ann. 1800, p. 226; Pollin. fl. veron. III, p. 32 et herb.!; Epipactis Segu. pl. veron. III, p. 253. - Icon.: Segu. op. et loc. cit. tab. VIII, fig. 10; Barla op. cit. tab 10, fig. 13-18.

Rarissima. Il Seguier loc. cit. dice che, in pineto Craccorum prope Volpianam ad Bolcae montis radices reperitur; il Pollini nella Flora cita questa pianta sulla fede del Seguier, ma non dice di averla raccolta od osservata: si trova nel suo erbario, ma senza indicazione di luogo. Non mi è mai calpitato di visitare la stazioue indicata dal Seguier jer questa pianta, nel tempo in cui la stessa è in fioritura; non sono pertanto in 
grado di dire se la stessa vi si ritrovi ancora in oggi. - La Goodyera repens cresce del resto sul M. Baldo, ed il Chiarissimo Antonio Manganotti la trovò nella Valle di S. Zeno. - Luglio ed agosto. 20

Io ho raccolto la Goodyera repens nella Naunia alla Mendola; nella stazione stessa indicata dal Chiarissimo Ambrosi,

\section{Tribus 3. - Malaxideae.}

\section{Gen. VIII. - Corallorhiza.}

78. Coralloriza innata $R$. Brown in Ait.h. liew. V, p. 209; Bertol. fl. it. IX, p. 635; Ambros. Al. Tir. austr. I, p. 738; Vis. et Sacc. cat. p. 59; Ophrys Corallorhiza L. sp. pl. 1336; Cymbidium Corallorhizon Swartz in act. holm. anm.1800, p. 238; Pollin. fl. veron. III, p. 38 et herb.! - Icon.: Barla op. cit. tab. 10, fig. 19-23.

Rara. Oichidacea rizomatosa ed arriza vivente specialmente nei boschi di Faggi ed anche di Mughi. - Nel M. Baldo in Noveza! ed ai Lavacci!; nei Lessini presso Chiesanova nel Bosco grande!, alle Scandole (e Pollini)!, ai Trachi e Bocca di Selva!, a Revolto! (e Caro Massalongo). - Da giugno a luglio. 2

Il fusto della pianta raccolta da me nel Veronese oscilla fra 9 e 22 centimetri, la spiga compresa. La intera pianta presenta nelle sue parti una tinta decisamente giallastra, qualche volta color mattone, senza sfumatura alcuna di verde: esemplari provenienti da altre stazioni italiane e forastiere concordano coi miei. La figura del Barla loc. eit. non mi sembra rappresenti fedelmente questa bella specie.

Il Chiarissimo Pollini nel Catalogo delle piante dell' Oito botanico Veronese per l'anno 1814 con un cenno di varie piante nuove (Verona. Tip. Mainardi), cita una Ophrys callirhiza da lui considerata come specie nuova e scoperta in sylvis abietineis montium Veronensium prope vicum Ecclesiam novum, e definita parvula Ophrydis species radice singulari praedita. Io non ho potuto trovare traccia di questa pianta in alcuna delle altre opere di Ciro Pollini: di quale specie intenda parlare non posso sapere, ma mi è nato il dubbio che la stessa possa essere benissimo la Corallorhiza innata, che per lo appunto si incontra nei pressi di Chiesanova; la forma precisamente singolare del rizoma di questa Orchidacea verrebbe in mio appoggio: seppure non traduciamo la parola singulari in unica, una sola; nel qual caso il Pollini avrà forse voluto accennare ad una qualche forma della Ophrys monorchis $L$.: però reputo più probabile la prima versione. 


\section{Tribus 4. -- Ophrydece. \\ Subtribus 1. - Gymnadenieae.}

Gen. IX. - Herminium.

79. Herminium clandestinum Gren. et Godr. fl. de Fr. III, p. 299; Herminium Monorchis R. Brown in 1 it. h. lew. ed. 2, V, p. 191; Bertol. fl. it. IX, p. 578; Ambros. fl. Tir. ausitr. 1, p. r19; Parlat. fl. it. III, p. 391; Vis. et Sacc. cat. p. 58; Ophrys Monorchis L. sp. pl. 1312; Pollin. viag. p. 113, 120 et fl. veron. III, p. 23 et herb.!; Orchis Monorchis $A l l$. $f l$. ped. II, p. 118; Orchis trifolia, floribus spicatis, herbaceis Segu. cat. p. $r 7$ et pl. veron. 1I, p. 131; Monorchis montana, minima, flore obsoleto, vix conspicuo Segu. pl. veron. III, p. 251; Moren. herb. - Icon.: Segu. pl. veron. II, tab. XVI, n. 15 et III, tab. VIII, fig. s; Barla op. cit. tab. 11, fig. 1\%-27.

Nei pascoli e nei luoghi boschivi dalla regione alpina alla montana; qualche volta isolata, più frequentemente gregaria: però non è comune. - Nel MI. Buldo nei pascoli della Valfiedda!, nella valle del Bastion!, nella val Brutta! (e Pollini), e secondo lo stesso Pollini in Campedello, alla Lonza, ai Lavacci, nel Pian della Cenere; nei monti Lessini agli Spiazzoi! e nella discesa da questo luogo al vajo di Squaranto!, e quindi ai Merli!; nel M. Zeola (A. Massalongo!) ecc. - Fiorisce in giugno e luglio. $2_{0}^{\circ}$

Il Seguier scrive di aver raccolto questa pianta presso Verona, nelle sabbie dell'Adige in vicinanza del Porto S. Pancrazio: si tratta evidentemente di un qualche individuo quivi trascinato ed abbandonato dalle acque, forse in seguito ad una piena del fiume.

Questa Ofridea varia assai per la altezza del fusto e per il numero delle foglie: i sinonimi del Seguier accennano manifestamente, il primo alla forma più lussureggiante munita qualche volta di tre ed anche di quattro foglie, ed il secondo alla forma a due foglie con il fusto cortissimo e la spiga formata di pochi fiori. Siccome in questa specie possono trovarsi da uno a tre ingrossamenti tuberiformi, ho preferito la denominazione specifica di clandestinum proposta dai Signori Grenier e Godron, all'altra di Monorchis, per verità antichissima e generalmente accettata dagli antori, ma in contraddizione col fatto.

Nelle frasi Orchis di molte sorte (Calceolari Viag. di M. B. p. 10), e molte specie di Orchis (Pona M. B. p. 190), secondo ogni probabilità devono intendersi compendiate, almeno nella massima parte, le Orchidacee appartenenti alla tribù delle Ofridee le quali vivono sul M. Baldo. 


\section{Gen. X. - Bicchia.}

80. Bicchia albida Parlat. R. it. III. p. 397 ; Satyrium albid.um L. sp. pl. 1338; Orchis albida Scop. fl. carn. ed. 2, II, p. 201; All. fl. ped. 1I, p. 149; Pollin. viag. p. 113 et fl. veron. $I I I, p$. ¿1 et herb.!; Gymnadenia albida C. L. Rich. in mém. du Muséum IV, p. 5\%; Ambros. fl. Tir. anstr. I, p. r02; Vis. et Sacc. cat. p. 56; Peristylus albidus Lindl. orchid. p. 299; Bertol. fl. it. IX, p. 572; Pseudo-oj'chis alpina flore herbaceo Segu. pi. veron. III, p. 254; MIoren. herb. - Icon.: Segu. pl. veron. III, tab. VIII, fig. 11; Barla op. cit. tab. 11, fig. 1-16.

Nei prati e nei pascoli della zona alpina e subalpina dai confini della provincia Veronese col Vicentino, al versante occidentale del $M$. Baldo; scende qualche volta nella regione montana al disot to dei 1000 metri: però non è pianta comune. - Qua e là in tutta la catena del M. Baldo, ai Colonei!, Montesel!, nella Valfredda! (e Seguier), in Cerbiol!, ai Lavacci (Pollini), nel Pian della Cenere! ove è pure stata segnalata da Pollini, Manganotti, Kellner; nei M. Lessini in Podesteria! (e Pollini), Maléra!, Spiazzoi! ecc.; nel M. Campobrun! (e Pollini Tierb.!); nel M. Alba (Bordoni sec. Seguier, Pollini); presso la Giazza (A. MIassalongo!). Giugno e luglio. 2

\section{Gen. XI. - Grinnadenia.}

81. Gymmadenia conopea $R$. Brown in Ait.h. kew.ed. 2, V, p. 191; Bertol. fl. it. IX, p. 562.; Ambros. 月. Tir. austr. I, p. 699 ; Parlat. fl. it. III, p. 400 ; Vis. et Sacc. cat. p. 56 ; Orchis conopsea L. sp.pl. 1335; Pollin. viag.p. 96, 119, 128, f. veron. III, p. 119, excl. syn. Segu. Orchis montana purpurea odurata et herb.!; Fr. Fontana cat. p. 32; Orchis palmata minor, calcaribus oblongis Segu. pl. veron. III, p. 251; Moren. herb. - Vernacolo: Erba della man, Manine, Zattelle, Zattine! - Icon.: Segu. pl. veron. III, tab. VIII, fig. 7; Barla op. cit. tab. 12, fig. 1-20.

$\beta$ albiflora. - Gymnadenia conopsea var. b. Parlat. $f$. it. III, 3. 101; Orchis ornithis Jacq. fl. austr. II, p. 23 (in icone tab. 138, radices erronee tuberiformes pictae). - Icon.: Barla op. cit. tab. 12, fig. 21-26. 
Volgatissima nei luoghi erbosi e freschi della intera provincia: dalla regione subalpina e montana sale alla zona alpina, scende nelle valli e si spinge a tutta la pianura, incontrandosi frequentissima nei prati e pascoli umidicci, lungo le sponde dei corsi d'acqua, ed anche nei luoghi boschivi ombrosi specialmente montani. - Secondo Seguier ( $p l$. veron. III, p. 251), in Mantica sylva sub maii mensis finem florens invenitur; Ciro Pollini nell'erbario la segnala nel $M$ r. Baldo, nei M. Lessini presso Velo e Chiesanuova, nel M. Pastello e nell'Agro Veronese presso Valeggio!; A. Massalongo in Vestena e nel M. Bolca!; Gregorio Rigo nei prati presso Rivole veronese, a $S$. Zeno di Montagna nel $M I$. Baldo ed in altri luoghi. Io per parte mia la ho raccolta, ovvero osservata copiosissima nella intera catena del $M$. Baldo, dei Lessini e dei monti che ne dipendono!; nella formazione montagnosa che separa il Vicentino dal Veronese!; nella valle dell'Alpo!; nella valle d'Illasi!; nella valle dell'Adige in vicinanza di Rivole!, ed alle sponde stesse del fiume ai piedi dei monti S. Marco e Cordaspina presso la contrada Casetta al disopra del porto della Perarola!; sulle rive del Benaco presso Colà!, Lazise!, ove venne pure indicata da Francesco Fontana ecc.; e nella pianura nei prati di Vigasio!, Bovolone!, Isola della Scala!, Sanguinetto!, Legnago! ecc. -- La varietà a fiori bianchi si incontra, però non molto frequentemente, assieme alla forma normale. - Da maggio a luglio secondo la altitudine della stazione la quale oscilla fra meno di $10 \mathrm{~m}$. e circa $2000 \mathrm{~m}$. sul livello del mare. 2

In questa specie $\mathrm{i}$ fiori mandano per lo più un odore gratissimo come di vaviglia, ma qualche volta sono inodori affatto $(G$. inodora Fries): variabili pure, a seconda dei luoghi, sono l'altezza del fusto, la larghezza delle foglie, la grossezza e compattezza della spiga, la grandezza dei tepali, la: lunghezza dello sperone, il quale però è sempre più lungo dell'ovario. Frequentemente ho osservato delle forme, le quali sembrauo quasi intermediarie fra la specie della quale è parola, e la seguente: non di rado la $G$. conopea e la $G$. odoratissima vivendo in società, non è impossibile il loro connubio, e la procreazione di susseguenti forme ibride. Il Seguier ( $p l$. veron. III, p. 251) scrive che: « Ex Alba monte varieta" tem detulit Bordonius, cujus calcaria brevioria, tum floris color ex albo " in rubrum vergebat; coetero eadem facies: » la varietà raccolta dal Bordoni potrebbe per lo appunto appartenere ad alcuna di tali forme intermediarie e forse ibride.

Il Pollini (viag. p. 96 et fl. veron. III, p. 124) riporta la Orchis montana purpurea odorata iel Segnier (cat. pl.p. 96 et pl. veron. II, p. 124) quale sinonimo della Orchis (Gymnadenia) conopea: il Par'atore ( $f$. it. III, p. 410) lo riferisce pure, ma dubitativamente, e sulla fede del Pollini. Il Seguier così descrive la sua pianta: "Flores in spicam longam dispo* nuntur, omnino purpurei, longo calcari donati: et Fuchsiana icone haud * male expressi. Odoris fiagrantia ab aliis speciebus facile distinguitur. * Julii mensis initio, dum colles Gretianenses peragrarem mihi occurrit, " rara tamen in agro veronensi: » ma nel contesto di questa diagnosi non trovo dati sufficienti per affermare che volesse effettivamente parlare 
della G. conopea: anzi il sinonino del Fucsio Orchis femina major e la figura di questi citata da Seguier darebbero a vedere il contrario; perchè nella figura del Fucsio gli ingrossamenti tuberiformi sono interi non divisi. Mi confermo in questi apprezzamenti osservando che nelle $p l$. veron. il Seguier inserisce la sua Orchis montana purpurea fra la 0 . militaris pratensis (O. ustulata) e la O. Morio mas foliis maculatis (O. mascula): il Pollini fu probabilmente indotto in errore dai caratteri desunti dalla lunghezza dello sperone e dalla fragranza dell'odore emesso dai fiori, i quali si riscontrano pure in altre specie; ed in me è nato il sospetto che la pianta del Seguier possa riferirsi alla O. fragrans Pollin., $\mathrm{i}$ cui fiori sono per lo più interamente porporini, disposti in spiga allungata, soavemente odorosi, muniti di uno sperone assai lungo, talchè frequentemente uguaglia in lunghezza l'ovario. Ad ogni modo è assai difficile sentenziare in modo assoluto quale pianta siasi realmente voluta indicare dal Seguier; nè escludo possa trattarsi di alcuna fra le tante forme ibride che effimere ed accidentali compaiono d'un tratto, rendendosi poscia invisibili per una lunga serie di anni: infatti il Seguier la dice rara nel Veronese.

82. Gymnadenia odoratissima C. L. Rich. in mém. du $M I u$ séum. IV, p. 57; Bertol. fl. it. IX, p. 561; Ambros. fl. Tir. austr. 1, p. 701; Parlat. fl. it. III, p. 402; Vis. et Sacc. cat. p. 56; Orchis odoratissima L. sp. pl. 1335; All. fl. ped. II, p. 150; Pollin. viag. p. 99, 122 et fl. veron. III, p. 250 et herb.!; Fr. Fontana cat. p. 32 ; Orchis palmata angustifolia, minor odoratissima segu. pl. veron. III, p. 250; Moren. herb. - Vernacolo: i nomi stessi della specie precedente. - Icon.: Segu. pl. veron. III, tab. VIII; fig. 6 ; Barla op. cit. tab. 13, fig. 1-15.

$\beta$ floribus roseis Parlat. fl. it. III, p. 403.

$\gamma$ floribus albis Parlat. fl. it. III, p. 403.

$\delta$ Idae. - Planta pumila, cum spica $10-12 \mathrm{~cm}$. longa : " spica laxa, pauciflora: bracteis flores aequantibus vel su«perantibus: foliis angustissimis. .

Cresce abbondantissima nei luoghi selvatici e rupestri, nei pascoli, nei prati, fra $\mathrm{i}$ frutici e gli arbusti delle zone alpina, subalpina e montana, dalle quali scende nei colli e nella pianura; nelle stazioni più basse ed a fioritura più precoce, frequentemente vive in società con la precedente; nelle stazioni più elevate la sua fioritura è posteriore a quella della G. conopea. - In tutta la catena del M. Baldo!; in Pravazar, Ime, Valfredda, Naole, la Ferrara, Valbrutta, Lonza....... salendo insino alle creste più elevate, nonchè in tutte le valli che solcano il versante occidentale fra i crepacci delle rupi e nei luoghi erbosi!; nei $M$. Lessini lungo tutta la catena!; nel M. Posta!, Campobrun!, Passo della Lora!; 
M. Zeola! (e Seguier e Pollini); M. Alba! (e Seguier e Pollini) ecc.; nei colli Benacesi, nei prati di Pezzoi sopra Torri (Rigo), ed in luoghi acquitrinosi alla Volpara!, ed alla Bagatta presso Lazise (Pollini, Fr. Fontana) ecc.; nella Valle dell'Adige sotto Rivole! ed alle sponde del fiume sotto la contrada Casetta! in società con le G. conopea, Orchis fragrans, Anacamptis pyramidalis, Serapias longipetala; nella Valle d'Illasi sin presso Tregnago (A. MLassalongo!); nella pianura Veronese nei prati di Vigasio! ecc. ecc. Le var. $\beta$ e $\gamma$ crescono promiscuamente alla forma tipica; la seconda si incontra assai raramente. La var. \& è stata primieramente da me trovata il 10 agosto 1872 sulle cime dei monti Laures e Macajon nella Naunia (Tirolo meridionale) fra 1500 e $1800 \mathrm{~m}$. di altitudine; recentemente in riva dell'Adige al piede delle ultime pendici del M. Baldo: si distingue dalla forma tipica per le minori proporzioni di tutte le sue parti, per la spiga composta da pochi fiori e distanti, per le foglie lineari e strettissime, per le brattee che uguagliano o superano in lunghezza $\mathrm{i}$ fiori. - Io dedico questa graziosa piantina alla pia e santa memoria di Ida de Betta, coltissima signorina veronese, rapita da morbo crudelissimo all'affetto dei suoi in Fondo della Naunia negli ultimi giorni dell'agosto 1872. - Fiorisce da maggio a settembre secondo la altitudine; nel 1875 io la ho raccolta nella Val grande di $M$. Baldo $(2100 \mathrm{~m}$.) nel mese di settembre, ed assieme a diverse altre specie alpine, in una buca dalla quale la neve, che in essa esisteva accumulata a memoria d'uomo, scomparve in quell'anno. 2

La Gymnadenia odoratissima, al pari della specie precedente, varia assai per l'altezza del fusto, per la spiga più o meno lunga e fitta, per la lunghezza dello sperone, ecc. Qualche volta i fiori inferiori sono assai discosti dai rimanenti, ed in tal caso le loro brattee assumono quasi un aspetto ed una consistenza fogliacea. - Nell'erbario Polliniano, assieme agli esemplari di G.odoratissima, trovasi pure un campione di Bicchia albida.

\section{Gen. XII. - Coeloglossum.}

83. Coeloglossum viride Hartm. fl. scand. ed. 1, ann. 1820, p. 329; Parlat. fl. it. III, p. 408; Vis. et Sacc. cat. p. 56; Satyrium viride $L . s p . p l$. 1337; Orchis viridis Crantz fasc. 6, ed. 2, p. 491; All. fl. ped. II, p. 150; Pollin. viag. p. 9s, 10s, 121 et fl. veron. III, p. 20 et herb.!; Gymnadenia viridis Ambros. fl. Tir. austr. 1, p. 703; Peristylus viridis Lindl. orchid. p. 299; Bertol. fl. it. IX, p. 5\%0; Orchis palmata, odore gravi, ligula bifariam divisa, flore viridi Segu. cat. p. 7 r et $p l$. veron. II, p. 133; Moren. hert. Vernacolo; Manine!, Zattine!. - Icon.: Segu. pl. veron. II, tab. XV, n. XVIII et tab. XVI, n. 18; Barla op. cit. tab. 13, fig. 16-19. 
Nei luoghi erbosi della regione alpina e subalpina scendendo dalle creste più elevate dei monti al disotto dei $1200 \mathrm{~m}$. - Lungo tutta la catena del $M$. Baldo, in Ime e Val Andrine!, Valfredda! (e Pollini), Naole!, Costabella! (e Rigo), la Lonza!, Novezina!, Noveza!, Artillon!, Altissimo di Nago! (e Pollini), M. Laste (Pollini), M. Cerbiol! ecc., e nei pascoli e nelle valli che solcano il versante occidentale in Ortigara! (e Pollini), Val Vaccara (Rigo), Valgrande!, Valle degli ossi! (e Tonini!) ecc.; nei Lessini alla Sega!, Podesteria!, Sparaver!, Malèra!, Spiazzoi! Revolto! ecc.; nel M. Posta!, Campobrun!, Passo della Lora!; M. Zeola! (e Pollini); M. Alba! (e Pollini) ecc. - Da maggio ad agosto e settembre secondo la altitudine e le condizioni locali. In Ime di M. Baldo ho trovato un esemplare in piena fioritura alla metà di aprile nel 1875 . 2

Questa specie varia assai per la statura, per il numero dei fiori che formano la spiga, per la forma e la lunghezza delle brattee. I fiori non sono affatto verdi, ma bensì flavo-virides, come osserva il Reichenbach ( $f$. germ. exc., p. 120). Nell'agosto del 1874, nella Valle delle pietre in M. Baldo, ho raccolto una forma mostruosa nella quale i fiori, ad eccezione dell'inferiore, per abortimento erano ridotti appena a mozziconi informi; mentre le brattee assai sviluppate formavano come una specie di ciuffo alla sommità del caule.

Gen. XIII. - Platanthera.

84. Platanthera bifolia C. L. Rich. in mém. Muséum p. 57; Bertol. fl. it. IX, p. 56 ex ex parte; Parlat. fl. it. III, p. 411; Vis. et Sacc. cat. p. 56; Orchis bifolia L. sp. pl. 1331; Pollin. viag.p. 113, 116 et fl. veron. III, p. 4 (an. ex parte?) et herl.!; Gymnadenia bifolia Ambros. fl. Iir. austr. I, p. r04; Orchis alba, bifolia, minor, calcari oblongo Segu. cat. pl. p. 76; pl. veron. II, p. 128. - Vernacolo: Castagnòle. Icon.: Segn. pl. veron. II, tab. XV, n. X; Barla op. cit. tab. 11, fig. 1-15.

$\beta$ laxiflora Reich. fil. orchid. Europ. p. 121, tab. LXXVI; Barla op. cit. tab. 15, fig. 1-3.

Frequentissima nei pascoli e nei boschi della regione subalpina e montana, meno frequente nella zona alpina; scende benanco nei colli e nelle valli e si spinge nella pianura. Nel M. Baldo nei pascoli di Pravazar!, Ime!, Valfredda!, la Ferrara!, Cambrigar!, Campedello! (e Pollini), la Ionza!, Noveza (Pollini), Artillon (Pollini) ecc., e così pure sul versante occidentale; nei Lessini presso Chiesanova! ecc. e in tutti i monti finitimi a questi o dipendenti da essi: Abramo Massolongo la raccolse a Cientro - Tregnago!, Ciro Pollini presso Lazise sul Benaco e nell' Agro veronese presso Guastalla, l'arciprete Masè nella pianura a S. Pietro in Valle, ove venne pure raccolta dal Pollini (herb.!). La varieta $\beta$ cresce promiscua- 
mente alla specie nei pascoli e nei prati del $M$. Baldo e dei Lessini. Da maggio a luglio 20

In questa specie è assai variabile la forma delle foglie, le quali sono più o meno allungate ed attenuate alla base, ottuse o quasi acute, talvolta assai larghe e quasi elittiche (9 cent. lungh. $-3,5$ cent. largh.), altra volta quasi lanceolato-lineari (10 cent. lungh. $-1,8$ cent. largh.) e talmente attenuate alla base da sembrar quasi prolungate in un picciuolo assai lungo.

85. Platanthera chlorantha Custor. ap. Reichb. in IMösl. handb. II, p. 1565 et fl. germ. excurs. p. 120; Parlat. fl. it. III, p. 413; Vis. et Sacc. cat. p. 56; Orchis virescens Gaud. fl. helv. V, in app. p. 497; Gymnadenia chlorantha Ambros. fl. Tir. austr. I, p. 705. - Icon.: Reichb. fil. orchid. tab. 430; Barla op. cit. tab. 15, fig. 4-17.

Meno frequente della precedente, insieme alla quale per lo più cresce in società. L'amico Gregorio Rigo la ha raccolta nei boschi montani del M. Baldo ai Cervi, fra Borno e la Prà; nello stesso monte io la ho trovata nei pascoli di Pravazar!; nei M. Lessini presso Chiesanuova!; ed in altri luoghi. - Fiorisce nella stessa epoca della precedente. 20

\section{Gen. XIV. - Traunsteinera.}

86. Traunsteinera globosa Reichb. fl. saxon.p. 87; Parlat. fl. it. III, p. 416; Orchis globosa L. sp. pl. 1332; All. fl. ped. II, p. 146; Pollin. viag. p. 108, 119 et fl. veron. III, p. 5 et herl.!; Fr. Fontana cat. p. 32; Bertol. fl. it. IX, p. 520; Ambros. fl. Tir. austr. 1, p. 6\$5; Orchis carnea, spica congesta, brevi calcari Segu. cat. pl. p. 77 et pl. veron. II, p. 129. - Vernacolo: Castagnòle. - Icon.: Barla op. cit. tab. 16, fig. 1-23.

$\beta$ humilis. - Planta pusilla, 16-17 cent. alta : caule - tenuissimo, triphyllo; folio inferiori lineari-lanceolato, an- gustissimo, ceteris bracteiformibus. Spica pauciflora; flores - exigui. .

Frequente nei prati e nei pascoli dei monti veronesi nei quali si innalza al disopra dei 2000 metri; scende pure nella regione dei colli; più raramente si incontra nella pianura, nella quale però sino ad oggi a me non venne ancor dato di osservarla. - Nel $M$. Baldo cresce lungo la intera catena; la ho osservata e raccolta nei pascoli dei Colonei, nella valle di Naole ove fu pure trovata dall'amico Rigo; indicata dal Seguier 
in radicilus jugi Costabella, fu rinvenuta nei pascoli che coronano questa cresta dallo stesso signor firgo e da me; cresce pure all' Altissimo di Nago! (Pollini, Ambrosi), nel M. Laste (Pollini), in Cerbiol!, nella catenella che correndo parallelamente all'Adige si distende dal M. Cornè sopra Brentino e la Corona, alla punta dei Lavacci al disopra di Avio. Nel complesso pertanto troviamo questa Orchidacea fra 1500 e 2100 metri di altitudine: e più al basso in Ime!, Valfredda! (e Seguier), Pravazar! ove è copiosissima, la Lonza!, Novesina!, i Zocchi (Pollini) ecc. Nei Lessini troviamo la Traunsteinera globosa al Corno d'Aquitio!, alla Sega!, in Podesteria!, sul Malèra!, ai Parpari!, presso Cliesanuova! ecc. E così pure nei monti che separano il Vicentino dal Veronese, p. e. nel M. Loblia!, M. Alba!, S. Bartolomeo Tedesco! ecc. Il professore Abramo Massalongo la raccolse nella Valle d'Illasi al II. Barbara, e presso Badia Calavena!; il chimico-farmacista Francesco Fontana nei colli Benacesi presso Lazise; infine Ciro Pollini scrive di averla trovata presso Verona nel Bosco Mantico e nel Pestrino - due stazioni nelle quali oygidì questa pianta è sicuramente scomparsa; della seconda localitì esistono esemplari nell'erbario!. La mia varietà $\beta$ rappresenta la forma tipica, si può dire, in miniatura; io ho raccolto tre esemplari di questa elegante piantina nei Mr. Lessini sulla cima del Malèra. - Da maggio a luglio secondo la altitudine. 2

Negli esemplari veronesi il caule di questa Orchidacea talora raggiunge $\mathrm{i}$ cinque o $\mathrm{i}$ sei decimetri ed anche più. La spiga, che è per lo più ovata, qualche volta si allunga e diventa quasi cilindrica; non oso affermare se a questa forma debba ascriversi un esemplare esistente nell'Erbario Polliniano colla scritta O. globosa var. ex sylva del Pestrino spica longa, ovvero se si tratti di una specie diversa.

\section{Subtribus 2. - Angiadenieae.}

\section{§1. - Monadenieae.}

Gen. XV. - Serapias.

87. Serapias Lingua L. sp. pl. 1314 ex parte; Pollin. fl. veron. III, p. 29; Vis. et Sacc. cat. p. 58; S. oxyglottis Bertol. fl. it. IX, p. 605. - Icon.: Barla op. cit. tab. 1\%, fig. 1.24.

Rarissima. L' Illustre Prof. Abramo Massalongo ha scoperto questa specie presso Centro!; essa si trova fra le piante di lui che si conservano nel Civico Museo: inoltre nell'Erbario del Chiarissimo P. A. Saccardo, Professore di Botanica nella R. Università di Padova, esistono esemplari raccolti dallo stesso A. Massalongo nel $M$. Baldo. Io sino a questo giorno non mi sono imbattuto in questa pianta; bensì ho trovato recentemente in val d'Adige, e sulla destra del fiume, alcune forme che po- 
trebbero ritenersi intermediarie fra la stessa e la specie che segue; e che per molti caratteri non esiterei punto riferire alla $S$. Lingua, se non fossero le due callosità esistenti alla base della lacinia mediana del labello che sono nettamente distinte.

88. Serapias longipetala Pollin. fl.veron.III, p.30; Gren. et Godr. fl. de Fr. III, p. 2r8; Parlat. fl. it. III, p. 424; Reichb. fl. germ. excurs. p. 130; S. Lingua L. sp. pl. 1344 ex parte; Pollin. herb.!; Bertol. fl. it. IV, p. 600, var. a et excl. syn. S. oxyglottis Tod.; Fr. Fontana cat. p. 32; Ambros. fl. Tir. austr. I, p. 711. var. $\alpha$; Zersi cat. p. 202, var. $\alpha$; S. pseudocordigera Moric. fl. venet. I, p. 574; Koch syn. fl. germ. et helv. ed. 2, p. 799 ; Car. prodr. fl. tosc. p. 598; Orchis montana italica, flore ferrugineo, lingua oblonga Segu. pl. veron. III, p. 248. - Icon.: Segu.pl.veron. III, tab. VIII, fig.4; Reichb. fil. orchid. tab. 441; Barla op. cit. tab. 18 et tab. 19. $\beta$ albiflora. - S. Lingua flore albo Fr. Fontana cat. $p .32$.

Copiosa nei luoghi umidi od acquitrinosi della provincia, tanto nel piano, come nei colli; più raramente nei monti. - Nell'Erbario del chiarissimo Professore Saccardo si consevano esemplari raccolti da Abramo Massalongo sul M. Baldo, ma sulla scheda non è indicata la stazione precisa della pianta; la quale si trova abbondante alle falde di questo monte tanto sul versante occidentale quanto sull'orientale: fu raccolta dal Sig. Gregorio Rigo nei prati acquitrinosi che sono sotto Rivole $(140 \mathrm{~m}$.); da me in questa stessa stazione e in riva all'Adige presso la Casetta sotto ai monti S. Marco e Cordaspina!; lo stesso Sig. Rigo la segnala nei prati di Pezzoi sopra Torri del Benaco: seguendo la riva del lago ed il corso del Mincio la troviamo presso Lazise (Pollini, Fr. Fontana, De Bracht, Rigo), a Peschiera! (Pollini, A. Massalongo), nel luogo detto il Paradiso!, a Salionze! (e Rigo!), a Valeggio (Pollini) ecc. Si trova pure nell' Agro e nella pianura veronese; presso Guastalla indicata da Pollini e ritrovata da me nei prati e nei luoghi torbosi lungo il corso del Tione!; nei prati al Bovo presso Cadidavid (A. Manganotti)!; presso Buttapietra (37 m.) lungo il Menago!; nei prati di Vigasio!, Isola della Scala! (30 m.) ecc. Il Pollini la raccolse pure nei colli presso Verona al Tagliaferro (herb. sub S. Lingua!); Seguier infine scrive che Bordoni la trovò nei monti presso Cogolo nella valle d' Illasi. Vire nella vicina provincia di Mantova (Pollin., Vis. et Sacc., Masè), nella Valsugana (Ambros.), nei prati del Trentino (fratres Perrini), nel Distretto di Rovereto (Facchini); lo Zersi infine la dice frequentissima fra il Benaco e Fozzolengo $(134 \mathrm{~m}$.). E rimarchevole che nella provincia Veronese questa bella specie si trora specialmente distribuita fra oltre i $200 \mathrm{~m}$. e meno di $30 \mathrm{~m}$. di altitudine alla parte occidentale della provincia, sopra una zona che dai piedi degli ultimi contrafforti del M. Baldo, si distende approssimativamente da $\mathrm{N}$ a S, allar- 
gandosi nell'Agro e nella pianura: è pure degno di nota che presso di noi la stessa sembra dimostrare una particolare predilezione per i terreni e le formazioni moreniche. - La var. $\beta$ fu scoperta da Fr. Fontana presso Lazise: forse la stessa corrisponde alla var. pallidiflora dell'Illustre Prof. Todaro. - Da aprile a giugno. 2

Della S. longipetala si conservano pochi esemplari nell' Erbario Polliniano, provenienti da località diverse, ed assai malandate, con la denominazione di S. Lingua: alcune schede portano la intestazione S. rubra, nessuna quella di $S$. longipetala. Ciro Pollini, che evidentemente confondeva in origine la vera $S$. Lingua con la specie della quale ora teniam parola, le separò all'epoca della pubblicazione del terzo volume della sua F'lora (a. 1824) creando la S'. longipelala: la qual cosa era gia stata fatta prima dal Moricand (a. 1820), descrivendo nelli Flora veneta I, p. 5r4 la S. pseudo-cordigera, che lo stesso Pollini poi riporta quale sinonimo. L'Allioni $f l$. ped. II, p. 148 nella sua Orchis Lingua ha realmente fuso insieme a questa la pianta corrispondente alla $S$. longipetala; dal momento che riporta il sinonimo di Seguier e cita la figura che lo stesso dà di questa pianta nella opera $p l$. veron. ecc.

La S. longipetala è pianta assai polimorfa e variabile per l'altezza e la robustezza del caule; per il numero, la grandezza e la tinta più o meno carica dei fiori; per essere questi più o meno approssimati o discosti; per la lunghezza e la larghezza del lobo mediano dellabello, per la lunghezza e la ampiezza delle brattee.

Formae hybridae.

Le specie del genere Serapias frequenternente vivono in società tra di loro e con diverse altre Orchidacee specialmente del genere Orchis. Tale promiscuità o coesistenza dà origine a diverse forme ibride: fra queste la più insigne è certamente la $S$. triloba Viv., alla quale non pochi fitografi si sforzano di ridurre il prodotto di tutti gli incrociamenti, dei quali uno o entrambi gli autori appartengono al genere Serapias. Queste forme sono generalmente rare, compaiono a lunghi intervalli, e per lo più in uno o pochi individui : della provincia veronese mi sono note le due seguenti, che certamente differiscono dagli esemplari di $S$. triloba favoritimi dall'amico Francesco Negri e provenienti da Castágnolo presso Pisa.

\section{Serapias Fontanae Rigo et Goir.}

- S. pedalis, foliis radicalibus quatuor lineari-lanceolatis - acutis, binis caulinis alternis, spica elongata laxa septem- flora, tepalis exterioribus oblongo-linearibus obtusis, binis 
- interioribus paulo minoribus obtusiusculis, omnibus li- beris, labello tepalis interioribus longiore a basi ad mar- ginem radiatim venoso, laeviter trilobo, inaequaliter cre- nato, lobo medio parvo ovato vel ovato-lanceolato obtuso, - gynostemio recto, bracteis ovarium duplo superantibus

- (4 cm. 1, $1 \mathrm{~cm} .1)$ : fibris radicalibus tuberosis.......

Questa forma ibrida venne raccolta dal sig. Francesco Fontana il 28 maggio, non si sa di quale anno, nei prati della Bottona vicino a Lazise. Il distinto botanico ed amico sig. Gregorio Rigo ne possiede un unico esemplare, avuto dallo stesso sig. Fontana, sul quale è stata compilata la descrizione che precede. Il sig. Rigo si è ripetutamente recato sopra luogo, per riutracciare questo ibrido, ma sempre infruttuosamente: sospettavamo da prima che la pianta del sig. Fontana fosse il risultato del connubio fra la Serapias longipetala e la Orchis laxiflora, la quale assieme ad altre specie del genere Orchis cresce abbondantissima nei prati della Bottona in società con la prima. Ma dopo maturo esame ci siamo convinti che l'esemplare da noi posseduto non risponde nè alla descrizione che i sigg. Grenier e Gudron fl. de Fr. III, p. 277 danno della Serapias longipetala-luxiflora, nè alla figura vella quale il sig. Barla op. cit. tab. 22 fig. 9-11 riproduce un ibrido delle Serapias longipetala $\theta$ Orchis laxiflora raccolto dall Ardoino presso Diano Marina (Flore des alpes maritimes p. 359). Opineremmo piuttosto che la pianta del Fóntana risulti dal conuubio della $S$. longipetala con la Orchis Morio, ma nella incertezza preferiamo intitolarla dal nome dello scopritore.

\section{Serapias Roselliniana.}

- S. spica sub-nutante 7-8ffora, floribus superioribus ap- proximatis, inferioribus remotiusculis; tepalis subaequali- bus, omnibus liberis, patentissimis; tribus exterioribus oblongo-lanceolatis, obtusis, binis interioribus e basi late - ovata attenuato-lanceolatis, obtusiusculis; labello tepalis - exterioribus sesquilongiore, callis duobus linearibus di* stinctis instructo, trilobo, lobis lateralibus patentibus, basi - truncatis, margine rotundatis, inaequaliter crenatis, lobo medio lateralibus sub-duplo longiore, laeviter incurvo, * subovato-lanceolato, apice rotundato, subintegro, glabro, - gynostemio recto; bracteis spathaceis, amplis $(47 \mathrm{~mm}$. l long., $12 \mathrm{~mm}$. lat.), ovato-lanceolatis, elongato-acuminatis, - flores aequantibus vel superantibus; cauli, superne rubente, cum spica $30 \mathrm{~cm}$. alto; foliis lineari-lanceolatis, longe acuminatis; fibris radicalibus duobus tuberosis subrotundis. ' 
Le brattee sono identiche a quelle della S.longipetala: i tepali, lunghi circa $20 \mathrm{~mm}$, sono di colore rossiccio sfumati di verde, gli esterni specialmente, e percorsi da linee longitudinali più cariche. Il labello è di un bel color rosso porporino rivo che contrasta con quello più sbiadito e chiaro dei tepali interni ed esterni; la sua lunghezza totale è di $30 \mathrm{~mm}$., la massima larghezza di $20 \mathrm{~mm}$.: i lobi laterali, lunghi quanto i tepali, presentano molte vene che dalla base corrono irradiando verso il margine, ramificandosi lungo il loro tragitto, specialmente in vicinanza del margine stesso: il lobo mediano è attraversato da 3-5 vene dalla base all' apice, ramificandosi esse pure orvero congiungendosi coi loro estremi prossimamente a questo.

Ho scoperto questa forma singolarissima (17 maggio 1882) nei prati presso Vigasio, nei quali colla $S$. longipetala vive buon numero di altre Orchidacee: erano due soli esemplari uniti insieme, con le fibre radicali intrecciate. - Ho creduto per un momento fosse il prodotto dello incrociamento della $S$. longipetala con la $O$. laxiflora. Ma anche in questo caso ho trovato che alla mia pianta non corrispondono nè la descrizione dei sigg. Grenier e Godron, nè la figura del Barla superiormente citate. Sospetto possa trattarsi di un ibrido fra la $S$. longipetala e la Orchis fragrans Pollin.: ma non volendo per ora avventurarmi a pronunziare un giudizio che sembrerebbe definitivo, consacro questa pianta alla memoria di Ferdinando Rnsellini, il maestro carissimo che seppe inspirarmi l'amore alla bella scienza.

Gen. XVI. - Chamaeorchis.

89. Chamaeorchis alpina C. L. Rich. in mén. Muséum IV, p. 57; Ambros. fl. Tir. austr. I. p. 717; Parlat. fl. it. III, p. 436; Goiran app. bot. in cron. alp. etc. p. 78; Vis. et Sacc. cat. p. 57; Ophrys alpina L. sp. pl. 1342; Pollin. fl. veron. III, p. 28 in adnot. ad gen. Ophrys, non viag.; Orchis alpina All. fl. ped. II, p. 149; Herminium alpinum Lindl. orchid. p. 305; Bertol. fl. it. IX, p. 579. - Icon.: Barla op. cit. tab. 23, fig. 14-20.

Rarissima. Ho scoperto questa vaza piantina, erborizzando assieme all'amico Caro Massalongo, negli ultimi giorni dell'Agosto 1874 in M. Baldo nei luoghi erbosi e fra i crepacci delle rupi nella Valle delle pietre, sul versante occidentale del monte a circa 2100 met.! - Fiorisce da luglio a settembre. 2

Ecco un nuovo punto nella catena delle alpi per definire più esattamente la linea di vegetazione di questa Orchidacea: la quale, copiosa in alcuni luoghi, rarissima in altri, cresce nei pascoli alpini della Carnia ed ai confini del Bellunese col Tirolo (Pirona, Vis. et Sacc.), in diversi Juoghi del Trentino e del Tirolo meridionale tedesco (Ambros., Facchin., Rota, 
Leyb.), sul M. Gazzo (Zersi), nel M. di S. Gottardo (G. Bauh., prodr. p. 29), nella Valsesia nell'Alpe Olen (Carestia il quale mi ha fornito molti esemplari), nelle alpi di Safau e Gressoney (Allioni), nelle alpi della Tarantasia (Bellardi), nel col de la Seigne (Webb herb.), nel Cenisio (Allioni il quale la indica ivi copiosissima), nella cima di Ronche (Negri), nelle Alpi marittime (Risso). Da questo cenno si vede che la Chamaeorchis alpina comparirebbe qua e là in tutta la cerchia delle alpi; per fermo le ricerche dei botanici alpinisti dimostreranno che la stessa deve ancora rinvenirsi in altri punti all'infuori di quelli ora citati.

Questa Orchidacea non è stata raccolta nè dal Pollini, nè da alcuno dei botanici che anteriormente e posteriormente a lui esplorarono il Veronese. Non so che possa essere la Ophrys alpina che il Pollini cita a p. $60,98,99,121$, del suo Viaggio; certamente non la nostra pianta: forse avrà voluto indicare una qualche forma pumila di Listera (Epipactis) ovata alla quale secondo Seguier e Pollini sarebbe da riferirsi l' Ophris - Ofride del Calceolari, che in tutto il Viaggio trovo citata una sola volta $p .116$ in Noveza, mentre si incontra frequente in tutta la catena del Baldo e precisamente nelle stazioni indicate a $p .98,99,121$. Ritengo però più probabile abbia voluto accennare ad una qualche forma di Coeloglossum viride (Orchis viridis $f$. veron.): infatti il Pollini pubblico il suo Viaggio, che doveva essere così acerbamente criticato, nel 1816; ora nel suo Erbario, e precisamente nel foglio che contiene la Orchis viridis, si trova fra le altre una scheda con la scritta Ophrys alpina, la quale è cancellata, e sopra alla cancellatura, che porta la data del 1819 , è scrittó non est: questa sclieda dimostrerebbe che sino al 1819 Ciro Pollini ritenne per la Ophrys alpina $L$. una qualche forma di Orchis viridis.

\section{Gen. XVIII. - Aceras.}

90. Aceras antrophora $R$. Brown in Ait.h. kew.ed. 2, V, p. 191; Reichb. fl. germ. excurs. p. 127; Parlat. fl. it. III, p. 439; Ambros. fl. Tir. austr. I, p. $75 t$ in app.; Ophrys antropophora L. sp. pl. 1343; Pollin. fl. veron. III, p. 24 et herb.!; Orchis antropophora All. fl. ped. II, p. 148; Orchis flore nudi hominis effigiem repraesentans mas Segu. cat. pl. p. 76; pl. veron. III, p. 126 non C. Bauh. pir. et excl. syn.; Moren. herb. - Icon.: Barla op. cit. tab. 23, fig. 1-13.

Scriveva Seguier: " in collibus urbi circumvicinis mihi semel occur- rit haec pulcherrima Orchidis species ( (pl. veron. II, p. 127); ब Ciro Pollini (fl. veron. III, p. 24) dice che < in collibus veronensibus raro occurrit $\gg$, probabilmente sulla fede del Seguier. Nell' Erbario Polliniano se ne conserva un esemplare unico!, e sulla scheda è scritto ubique vulgatissima, ma senza indicazione della località in cui venne raccolta la 
pianta, e con carattere che non è del Pollini, il quale la ebbe probabilmente dal Balbis: infatti nella Flora veron.l.c. scrive di avere ricevuti esemplari della specie in quistione da questo botanico. Io per pirte mia la ho replicatamente ricercata con ogni cura e pazienza, anco di recente: ma senza risultato alcuno. Il Parlatore la indica nel Veronese ma sulla fede del Pollini; e così pure, secondo ogni apparenza, l'Ambrosi : il Tonini, il Manganotti, il Rigo ne fecero essi pure ricerca ma infruttuosamente: sospetto che molto probabilmente la $A$. antropophora sia scomparsa dalla nostra Provincia. Del resto è pianta rarissima nel Veneto, dove ai giorni nostri è stata raccolta solo nei lunghi umidi dei Colli Euganei dal chiarissimo Trevisan; l'Ambrosi non la annovera fra le Orchidacee del Tirolo meridionale; non cresce nel Mantovano; lo Zersi la indica rarissina nel Bresciano in luoghi erbosi sopra Zone.

\section{Gen. XVIII. - Himantoglossum.}

91. Himantoglossum hircinum Spreng. syst. veg. 11I, p. 691; Bertol. fl. it. IX, p. 56s; Ambros. fl. Tir. austr. I, p. 697; Parlat. fl. it. III, p. 113; Vis. et Sacc. cat. p. 56; Satyrium hircinum L. sp. pl. 1337; Orchis hircina Scop. A. carn. ed. 2, II, p. 193; All. fl. ped. II, p. 116; Pollin. viag. p. 18, 110; fl. veron. III, p. 15 et herb.!; Fr. Fontana cat. p. 32; Orchis barbata, odore hirci, breviore latioreque folio Segu. cat. pl. p. 76; pl. veron. II, p. 121; Moren. herb. - Icon.: Segu. pl. veron. II, tab. XV, n. I; Barla op. cit. tab. 21, fig. 1-23.

Stando a quanto scrive il Seguier ( $p l$. veron., II, p. 121), ai tempi di questo botanico l' Himantoglossum hircinum doveva essere specie assai rara presso di noi: "Ex omnibus Orchidum speciebus quae in agro re- ronensi reperiuntur haec minus praesto est, mihique semel aut iterum - occurrit Junio mense circa Gretianam vicum, in eo loco quem indigenae * gli Sarmazzi vocant. » Oggidì si incontra assai frequente e copiosa tra il Benaco ed il confine vicentino, nei boschetti e nei pascoli di tutta la regione collina; è più rara nella montana; non mi venne mai dato di osservarla nella regione subalpina ed alpina. - Presso Soave (Follini); nella Valle d'Illasi alla Canova presso Tregnago (A. Massalongo!) alla Mosella!; nei colli di Montorio! (e A. Manganotti!); nel MI. Trezzolan!; nel Vajo di Squaranto!; nei Lessini presso Velo (m. 1292, A. Massalongo!); in tutta la Valle Pantena alle Stelle!, Vendri!, Novaglie!, Cellore!, Stalavena (Tonini!), Grezzana!, Quinto! ecc.; nei colli presso Avesa! (e Pollin. herb.!) ed altrove; nella Valle dell'Adige presso Rivole! ecc.; nella Valpolicella!; nel M. Pastello!; alle falde settentrionali del M. Baldo presso Castione (500 m., Pollini); nei colli Benacesi a Lazise (Fr. Fon- 
tana), Rocca di Garda (Pollini), Scaveaghe!, S. Vigilio (Pollini), Albisano (Rigo), Torri del Benaco (Rigo) ecc. - Fiorisce in maggio e giugno. 2

In questa specie la lacinia mediana del labello è lungamente o brevemente bifida, altre volte solo smarginata ovvero appena munita di piccoli denti. - Variabilissima è pure la altezza del caule e la grandezza delle foglie.

\section{Gen. XIX. - Anacamptis.}

92. Anacamptis pyramidalis $C$. L. Rich. in mém. du Muséum IV, p. 55; Parlat. fl. it. III, p. 451; Vis. et Sacc. cat. p. 56; Orchis pyramidalis L. sp. pl. 1332; All. fl. ped. II, p. 145; Poliin. viag. p. 18, 88, 119; fl. veron. III, p. 6 et herb.!; Fr. Fontana cat. p. 32; Bertol. fl. it. IX, p. 518; Ambros. fl. Tir. austr. I, p. 609; Orchis purpurea spica congesta pyramidali Segu. cat. pl. p. 77 ; pl.veron. II, p. 129; Orchis purpurea spica congesta, flore roseo Segu. l. c. - Vernacolo: Castagnola, Guardaprà, Supa (Monti Diz. bot. veron. p. 134). - Icon.: Segu. pl. veron. II, tab. XV, n. XI; Barla op. cit. tab. 26.

"Variat longitudine calcaris, ovarium sub-aequantis - vel superantis; floribus purpureis vel laete roseis et $\beta$ floribus albis Purlat. fl. it. III, p. 451.

Frequentissima dalla pianura alla regione montana, raramente nella subalpina; si innalza sin quasi ai 1200-1300 metri: ama i prati, i pascoli, i luoghi erbosi e soleggiati; fra i querceti la ho trovata in unione al Limodorum abortivum. - Nei prati di tutta la pianura: Legnago!, Villabartolomea!, S'anguinetto!, Isola della Scala!, Vigasio! ecc., in tutto l'Agro Veronese a Cadidavid!, Buttapietra!, Custoza!, Sommacampagna!, nei pascoli di Prebian fra Villafranca e Valeggio!, Castelnuovo!, Colà (e Seguier) ecc.; presso Verona e nella cerchia stessa della città p. e. nel giardino del Collegio degli Angeli!; in tutti i colli e monti prossimi a Verona!; nelle Valli dell'Alpo!, d' Illàsi! (e A. Massalongo!), nella Valle Pantena! (e Tonini!), nella Valpolicella!, nei Lessini e monti dipendenti!, nel M. Pastello!, nella Val d'Adige!, alle falde del $M$. Baldo da Bellumo veronese a $R$ ivole ecc. specialmente presso Brentino!, Preabucco!, Tessari!, Casetta!, Incanale!; lungo la sponda del Benaco da Malcesine a Peschiera!, e nei colli Benacesi alla Rocca di Garda (Rigo), Costermano (Rigo), Valle dei Molini (Rigo), Torri (Rigo); nel MI. Baldo nei pascoli di Pravazar!, alla Ferrara! ecc. - La var. $\beta$ a fiori bianchi è rarissima: l'amico Rigo la ha raccolta nei colli sopra Torri del Benaco. - Fiorisce dalla meta di aprile a tutto giugno secondo l'altitudine delle varie stazioni. 2 - L'Arciprete Masè ha pure raccolto questa specie ai confini del Mantovano col Veronese a Casteldario. 


\section{§ 2. - Diadenieae.}

\section{Gen. XX. - Nigritella.}

93. Nigritella angustifolia $C$. L. Rich. in mém. du Muséum IV, p. 56; Bertol. fl. it. IX, p. 573; Ambros. fl. Tir. austr. 1. p. 708; Parlat. fl. it. III, p. 527; Vis. et Sacc. cat. p. 57; Satyrium nigrum L. sp. pl. 1333; Orchis nigra Scop. fl. carn. ed. 2, II, p. 200; All. fl. ped. II, p. 150; Pollin. viag. p. 98, 108, 122; fl. veron. III, p. 20 et herb.!; Orchis palmata angustifolia, alpina, nigro flore Segu. cat. p. 77; pl. veron. II, p. 133; Moren. herb. - Vernacolo: Zattine!, Manine!. - Icon.: Segu. pl. veron. I1, tab. XV, n. XVII; Barla op. cit. ta\}. 27, fig. 17-30.

$\beta$ rosea Vis. et Sacc. cat. p. 57; Nigritella angustifolia var. $\beta$ Bertol. fl. ital. IX, 574; Parlat. fl. ital. III, p. 527 var. $\mathrm{b}$; Orchis nigra var. $\beta$ Pollin. $f$. veron. III, p. 21.

Dal confine della Provincia veronese colla vicentina al fianco occidentale del $M$. Baldo, frequentissima nei pascoli della regione alpina e subalpina, più rara nella montana. Nel M. Baldo ai Colonei!, Naole! (Pollini! Rigo), Costabella! (Rigo), Ime!, Valfredda!, Valbrutta!, Campedello (Seguier), Val Losana!, Noveza!, Cerbiol!, Dossioi!, Zocchi!, Altissimo di Nago! ecc. fra $2196 \mathrm{~m}$. e $1180 \mathrm{~m}$; nei M. Lessini al Corno d'Aquilio!, Corno Mozzo!, Podesteria!, Malèra (1873 m.)!, Passo della Pertica (1559 m.)!, Spiazzoi (1376 m.)!, ecc.; nel M. Posta!, Campobrun!, M. Zeola! (e A. Massalongo!), M. Alba! (c Pollini!) ecc. - La varietà a fiori rosei $\theta$ porporascenti è più rara, essa si trova qua e là assieme alla forma tipica: io la ho raccolta nel $M$. Baldo in Cerbiol!, nei M. Lessini agli Spiazzoi!, e nel M. Alba! - Fiorisce da giugno a luglio; nelle stazioni elevatissime anche in agosto e settembre. 2

La Nigritella suaveolens Koch è ritenuta forma ibrida nata dallo incrociamento della $N$. angustifolia e della Gymnadenia conopea, e da altri fra la prima e la $G$. odoratissima: ritengo col Parlatore, che sia ibrida ora delle prime ora delle seconde piante. E pianta rarissima, che sino ad oggi fu rinvenuta nel Veneto soltanto nei pascoli del Trivigiano e del Vicentino (Vis. et Sacc. cat. p. 57). Il Facchini la raccolse nei pascoli alpini di Val di Fassa. Le ricerche future dimostreranno probabilmente la esistenza di questo ibrido anche nelle alpi Veronesi. Nel mio erbario ne tengo un esemplare unico favoritomi dall'amico G. Cuboni che lo raccolse in Val Formazzo nelle alpi centrali. 


\section{Gen. XXI. - Orchis.}

Sub-genus 1. - Herorchis Reichb. fil. orchid. p. 11.

\section{Sectio 1. - Papilionaceae.}

94. Orchis rubra Jacq. collect. I, p. 60; All. auctar. p. 31; Pollin. herb.!; Ambros. fl. Tir. austr. I, p. 752 in App.; O. papilionacea Pollin. fl. veron. III, p. 1.3, excl. nonn. syn.; Vis. et Sacc. cat. p. 56; O. papilionacea b rubra Parlat. fl. it. III, p. 459. - Icon.: Barla op. cit. tab. 23, fig. 16-18.

Rara : nei luoghi erbosi secchi e nei pascoli sterili dell' Agro Veronese; eccezionalmente anche nella bassa pianura. - Il Pollini la raccolse nei pascoli del Bosco Mantico', e la indica pure nei prati presso Valeggio; A. Misssalongo presso Villafranca nei pascoli di Prebian!; A. Manganotti nei prati al Bovo presso Cadidavid!'; l'amico Rigo nei prati sotto Custoza!'; il farmacista Giovauni Navi presso Nogara! - Fiorisce da aprile a giugno. 20 - Caro Massalongo mi ha favorito esemplari raccolti nei colli Euganei: il Rigo mi scrive che vive copiosa nella finitima provincia Vicentina nei prati di S. Orso alle falde del $M$. Summano: cresce pure nel Bassanese, Trivigiano, Bellunese, nel Friuli, non che nel Bresciano, ma rara, e in quel di Bergano.

Il Pollini nella descrizione di queste specie dice bracteis ... germine longioribus: il che non è rigorosamente vero, perchè in generale le brattee sono sempre alqquanto più brevi dell' ovario (e tale è il caso dei suoi esemplari), od appena, nei fiori inferiori, sono talora un po'più lunghe o quasi uguali all' ovario.

\section{Sectio 2. - Moriones.}

95. Orehis Morio L. sp.pl.1333; Allion. fl. ped. II, p. 146, excl. syn. et icon. Seguieri Orchis Morio foemina, calcari extuberante, bifariamque diviso; Pollin. viag. p. 18, 88, 96, 119; fl. veron. III, excl. var. $\beta$; herb.!; Fr. Fontana cat. p. 32 ; Bertol. fl. it. IX, p. 521; Ambros. fl. Tir. austr. 1, p.686; Parlat. fl. it. III, p. 463; Vis. et Sacc. cat. p. 55; Orchis Morio foemina Segu. cat. pl. p. 76; pl. veron. II, p. 125; Moren. herb. - Vernacolo: Salep, Castagnole: il primo di questi due nomi è dato, più particolarmente dagli erbaiuoli, anzichè alla pianta intera, agli ingrossamenti tuberiformi. - Icon.: Segu. pl. veron. II, tab. XV, n. VII; Barla op. cit. tab. 30, fig. 1-32. 
- Stirps satis variabilis longitudine caulis, magnitudine - et coloratione florum, nec non spicae densitate. In ditione - nostra, praeter genuinam, sequentes occurrunt formae. ' $\beta$ picta Lois fl. gall. ed. 2, p. 161 (icon autem, tab. 26, monente cl. Parlatore, mala); O. Morio var. d Parlat. fl. it. III, p. 464; O. Morio var. picto-violacea Barla op. cit. p. 45. Icon.: Barla op. cit. tab. 31, fig. 1-\%. - Haec forma culta in horto ab amico J. Menegazzolio, rei herbariae cultore eximio, constanter faciem suam servavit.

$\gamma$ rosea; O. Morio var. c Parlat. fl. it. 1II, p. 464; O. Morio var. picta-rosea Barla op. cit. p. 45 et 46; O. Morio foemina, flore roseo Segu. pl. veron. III, p. 243; Moren. herb. Icon.: Barla op. cit. tab. 31, fig. 8-19.

o alba; O. Morio var. fl. albo Fr. Fontana cat. p. 32: O. Morio var. b Parlat. fl. it. 1II, p. 464; O. Morio var. picta alba Barla op. cit. p. 46; O. Morio foemina, flore albo Segu. pl. veron. 1I1, p. 244; Moren. herb. - Icon.: Barla op. cit. tab. 31; fig. 20-23.

foliis maculatis Parlat. fl. it. III, p. 464.

Frequentissima nei pascoli, nei prati aridi e secchi, nel margine dei campi, negli argini, nei dumeti ecc. della intera Provincia, ascendendo dalla pianura sin quasi alla zona subalpina del Baldo, dei Lessini e dei monti da essi dipendenti. - Nella pianura a Legnago!, Sanguinetto!, Cerea!, Isola della Scala!, Bovolone!, Vigasio! ecc.; nell'Agro veronese a Buttapietra!, Cadidavid!, S. Giovanni Lupatoto!, ecc.; presso Villafranca nei pascoli di Prebian!; a Custoza!, Guastalla!, Sommacampagna!, Castelnuovo! ec.; nel Bosco Mantico!; nei pressi di Verona, e nei fossi e negli orti della stessa città!; in tutti i colli!; nelle valli dell'Alpo!, di Roncà !, di Illàsi!, di Squaranto!, Pantèna!, Policella!, dell' Adige! ed in tutti i monti confinanti!; lungo lo sponde del Benaco ed in tutti i colli Benacesi!; nel $M$. Baldo dalle falde alla Ferrara!, Pravazar!, Ime! ecc. sin oltre a circa 1200 metri. La var. $\beta$ si incontra qua e là frammista alla forma tipica; recentemente la ho osservata presso Giare alle falde del $M$. Tesoro a poca distanza dal Ponte di Veja: però è assai rara. Le var. $\delta$ e $\gamma$ sono frequenti; Seguier le indica presso Verona, io le ho rinvenute nel Bosco Mantico!, nel Colle delle Ungherine!, al Masetto! ecc.: la var. $\gamma$ a fiori bianchi è pure segnalata dal Fontana a Lazise, e dall'anico Rigo nei pascoli dei colli Benacesi. Io non mi sono mai imbattuto nella var. a foglie macchiate: il prof. A. Massalongo la ha raccolta presso Tregnago nel $M$. Barbara e nel luogo chiamato Gazzà! - Fiorisce da marzo a maggio secondo l'altitudine. 20 - Gli erbainoli raccolgono gli ingrossamenti tuberiformi delle fibre radicali per smerciarli ai farmacisti ed ai droghieri. 
A torto Allioni e Pollini hanno riferito alla O. Morio la piantia del Seguier 0 . Morio foemina, calcari extuberante, bifariamque diviso cat. pl. p. 76 et pl. veron. II, p. 125, che invece spetta alla 0 . laxiflora. Noto però che fra le varie forme di $O$. Morio, ne ho trovata uva che offre lo sperone più lungo di quello che generalmente sia nella forma normale, alquanto ingrossato all'apice e quivi leggermente smarginato.

\section{Sectio 3. - Coriophorae.}

96. Orehis corioplisra L. sp. pl. 1332; All. fl.ped. II, p. 116; Pollin. herb.! et fl. veron. III, p. 6, var. $x$; Fr. Fontana cat. p. 32; Bertol. fl. it. IX, p. 522, ex parte; Reichb. fl. germ. excurs. p. 12.3; Gren. et Godr. fl. de Fr. III, p. 287, var. a; Parlat. fl. it. III, p. 463, var. b; Vis. et Sacc. cat. p. 55, var. $\alpha$; O. cimicina Crantz stirp. austr. VI, p. 498; Orchis odore hirci minor Segu. cat. pl.p. ry; pl.veron. II, p. 12s; Orchis spica purpurea foetida Segu. cat. pl. p. $7 \% ;$ pl. veron. II, p. 128. - Icon.: Barla op. cit. tab. 32, fig. 1-16.

- Variat colore florum fuligineo vel purpureo: spica teter- vime foetet (Seguierus loc. cit.). Legi quoque formam fibris - tuberosis radicalibus trinis praeditam. "

Poco frequente. Si incontra qua e là nei prati e nei pascoli umidicci od acquitrinosi ed anche secchi della pianura e dei colli: il Seguier però scrive che cresce in pascuis montis Baldi orientem versus. - Lo stesso Seguier dice di averla rinvenuta una sola volta nei fossi di Verona presso la porta di S. Zeno: Pollini la raccolse al Pestrino (herb.!) e presso $L a$ zise (e Fr. Fontana); G. Rigo nei prati umidi sopra Torri del Benaco: a me si è offerta in pochissimi esemplari, sola o mescolata alla specie seguente, nel Bosco Mantico presso Verona!, sulla destra dell'Adige ai piedi del monte S. Marco presso Incanale!, e recentemente in un prato presso Vigasio!. - Fiorisce in maggio e giugno. نे

97. Orchis fragrans Pollin. herb.!; cat. pl. hort. bot. veron. ad anmum 1812, p. 16; elem. bot. II, p. 155-157 (in exemplaribus selectis tantum); Reichb. fl. germ. excurs. p. 121; O. coriophora Pollin. fl. veron. III, p. \%, var. $\%$; Bertol. fl. it. IX, 5.2 ex parte; Gren. et Godr. fl. de Fr. III, p. 287, var. $\beta$; Ambros. R. Tir. austr. I, p. 681; Parlat. fl. it. III, p. 463, var. ; 0. Polliniana Sprengel pl. min. cogn. pug. 1I, p. 78; Pollin. hort. et prov. veron. pl. nov. vel min. cogn. fasc. I, p. 25. - Vernacolo: Castagnole. - Icon.: Pollin. elem. bot. 1I, tab. ultima, fig. 2; Barla op. cit. tab. 32, 
fig. 17-28. - Exsicc. Goir. ap. Société helvétique pour l'échange des plantes, anno 1880.

- Stirps egregia ab Orchide coriophora satis diversa, mo- nente Cl. Ludovico Reichenbach; qui tres enumerat di-

- stinctas formas: ,

$\alpha$ fragrans Pollin. - "Floribus densissimis, saturate - rubris. "

$\beta$ cassidea M. B. Al. taur. III, p. 60. - Floribus - laxis, pallidioribus, magnitudine illorum praecedentis. '

$\gamma$ grandiflora Reichb. fl. germ. excurs. p. 121. --- Spica - densa, floribus majoribus, pallide roseis. - Praeterea se- quens adjungenda est forma quae nuperrime mihi obviam - venit,

$\delta$ albiflora. - . Spica laxiuscula; floribus omnino albis. 。 - Variat quoque longitudine calcaris. Flores odorem flomun - Mespili monogynae et M. Oxyacanthae fortiter olentes (Pollin. - fl. veron. III, p. 7). - Stirps certissime haud proxima "Orchidi pyramidali ut ait Cl. Sprengel."

Copiosissima nei lúoghi erbosi e scoperti, nei pascoli e nei prati tanto umidi quanto secchi nella parte occidentale della Provincia specialmente: frequentemente gregaria, altre volte isolata, io la ho trovata non di raro in societa con la Epipactis palustris, Anacamptis pyramidalis, Gymnademia conopea, Gymnadenia odoratissima, Serapias longipetala, Orchis laxiflora, ecc. Si trova approssimativamente distribuita sopra quella stessa zona che segna l'area di vegetazione della. Serapias longipetala colla quale in molte stazioni cresce promiscuamente. - Presso Guastalla lungo il corso del Tione!; nei pascoli tra Villafranca e Valeggio (Pollin. herb.!), lungo il corso del Mincio! (e Pollini), a Peschiera!, presso Garda - Torri (Rigo), a Costermano (Gius. Fontana!) ed altrove nei colli Benacesi; nella Valle dell' Adige in vicinanza di Rivole!, e seguendo le sponde del fiume ad Incanale!, la Casetta! ecc. La var. $\propto$ è la più comune; le var. $\beta, \gamma, \delta$ sono assai rare: le ho raccolte recentemente in luoghi erbosi lungo l'Adige al piede dei monti S. Marco e Cordaspina! - Fiorisce in maggio e giugno. 2

\section{Sectio 4. - Militares.}

98. Orchis ustulata $L . s p . p l .1333 ; A l l$. fl. ped. II, p. 147; Pollin. viag. p. 88; fl. veron. III, p. 10 et herb.!; Fr. Fontana cat. p. 32; Bertol. fl. it. IX, p. 531; Ambros. fl. Tir. austr. I, p. 683; Parlat. fl. it. III, p. 472; Vis. et Sacc. 
cat. p. 55; Orchis militaris pratensis Segu. cat. p. 77; sp. pl. II, p. 123; Moren. herb. - Vernacolo: Castagnole. Icon.: Segu. pl. veron. II, tab. XV, n. IV; Barla op. cit. tab. 33, fig. 1-15.

$\beta$ pauciflora. - "Spica depauperata. "

Comunissima in tutta la Provincia dal limite della pianura e dai colli a tutta la regione montana; penetra benanco nella zona subalpina: si incontra nei luoghi erbosi, nei pascoli, negli argini ed anche nei luoghi boschivi. - A Legnago!, Isola della Scala! (e Masè), Vigasio! ecc.; fra Villafranca e Valeggio nei pascoli di Prebian!; Sommacampagna!, Custoza!, Guastalla! ecc.; nel Bosco Mantico!, Parona!, Avesa! ecc.; nei fossi della città di Verona!, in Campo Marzo!, al Pestrino! ove venne pure indicata da Seguier e Pollini, in tutti i colli!, nella Valle dell'Alpo!, Val d'Illàsi!, Valpolicella!, Val d'Adige!, Val di Caprino!; a Pastrengo (B. Pellegrini!), Costermano! ecc.; presso Lazise (Fr. Fontana); alle falde del Baldo presso Rivole!, Incanale! ecc., in Val Lunga presso Castion, Albisano, Marciaga (Rigo , e nello stesso monte alla Ferrara!, nei pascoli di Pravazar!, Ime! ecc.; nel M. Pastello! ; nei Lessini presso Velo (A. Massalongo!) ecc. La var. $\beta$ nel M. Trezzolan!. - Fiorisce da aprile a giugno secondo la altitudine. 2

Ho osservato esemplari col labello verdognolo. La lacinia mediana del labello qualche volta è intera, tronca o smarginata; generalmente biloba, ed in tal caso manca non di raro il piccolo dente che è situato frammezzo ai due lobi. - Recentemente ho osservato nei pascoli di Prebian, stazione nella quale vive buon numero di specie appartenenti al genere Orchis, usa forma che appare come intermediaria fra la O. ustulata e la 0 . tridentata: la spiga è quasi tonda come nella seconda non cilindracea come nella prima; $i$ fiori di un bel rosso porporino, piccoli, banno i tepali esterni che si avvicinano a quelli della 0 . tridentata, mentre il labello è prossimo a quello della 0 . ustulata. Forse un ibrido O. tridentata $\times 0$. ustulata?

99. Orchis tridentata Scop. fl. carn. ed. 2, 11, p. 190; Parlat. fl. it. III, p. 476; O. variegata All. Al. ped. II, p. 147; Pollin. fl. III, p. 11 et erb. sed specimina non veronensia!; Bertol. fl. it. IX, p. 534 ex parte et exclus. plerisq. syn.; Ambros. fl. Tir. austr. 1, p. 682; Vis. et Sacc. cat. p. 55; Orchis militaris, pratensis, elatior, floribus variegatis Segu. cat. p. 76 ; pl. veron. $I_{1}^{r}$, p. 123. - Vernacolo: Castagnole. - Icon.: Segu. pl. veron. tab. XV, n. III; Barla op. cit. tab. 31, fiq. 1-18.

$\beta$ sulcata. - " Spica congesta, intense purpurea. Cal- care sulco laevissimo percurso.

$\gamma$ candidissima. - Floribus candidissimis. 
Comunissima nei pascoli, nei prati, nei margini dei campi, negli argini, più raramente nei luoghi boschivi : dalla pianura e dai colli alla regione subalpina. Ho raccolto questa specie nella città stessa di Verona nel giardino del Collegio degli Angeli!, si trova dal resto in tutte le stazioni indicate più sopra per la $O$. ustulata, nè starò a fare una inutile ripotizione: basti il dire che nella pianura, nell'agro, nei colli, nei monti si incontra ad ogni passo, e più di quest'ultima si spinge in altitudine trovandosi abbondantissima nel M. Baldo nei pascoli di Pravazar!, Cambrigar!', Campedello!, Lonza! ecc. - La var. $\beta$ cresce nei pascoli del M. Baldo!: la spiga è piuttosto povera di fiori molto avvicinati, di un rosso porporino assai vivo: lo, sperone presenta un leggero solco dal quale è percorso in tutta la sun lunghezza: forma interessantissima da studiarsi ulteriormente. - La var. $\gamma$ è rarissima: ne ho trovato tre esemplari fra Villafranca e Valeggio nei pascoli di Prebian! - Recentemente ho osservato nel $M$. Tondo $(640 \mathrm{~m}$.) un unico esemplare, assai deturpato per le brinate del mese di aprile, che mi sembra potersi riferire alla Orchis tridentato-militaris Barla et Canut (Barla op. cit. tab. 34, fig. 16-26). - Dalla fine di marzo a tutto maggio ; in giugno nelle stazioni elevate. 2

La spiga è ricca, altro volte poverissima di fiori: questi presentano una tinta ora molto carica, altre volte pallidissima. Il labello è stato trovato in molti esemplari da me osservati anche più lungo dei tepali esterni : la forma delle lacinie mediana e laterali è variabilissima; queste sono più o meno larghe, cuneate o lineari, munite di denti brevi o lunghi ; in alcuni esemplari sono obliquamente troncate, interissime, prolungate in una specie di orecchietta dalla parte della lacinia mediana.

Il Pollini descrive nella Flora questa pianta ma non la indica in alcun luogo del Veronese, nè mai la nomina nel suo viaggio; anche nel suo Erbario nel foglio che contiene la $O$. tridentata, che è la sua $O$. variegata, non si trova alcun esemplare reronese: il chiarissimo Autore ha senza dubbio confuso la 0 . tridentata, almeno per quanto riguarda le piante veronesi, con qualche forma della $O$. militaris, con quella segnatamente a spiga ovata e povera di fiori. Infatti riferisce alla O. militaris il sinonimo di Seguier Orchis militaris, pratensis, clatior, floibus variegatis che a termini della descrizione e della figura datane dall'autore spetta sicuramente alla $O$. variegata. Anzi staudo a quanto si legge nell'Appendix in calce al III vol., p. 312 , si rende sempre più eridente lia confusione fatta dal chiarissimo autore.

100. Orchis tephrosanthos Vill. pl. du Dauph. II, p. 33, exclus. syn. Seguieri; Pollin. fl. veron. III, p. 11, exclus. syn. Segu., et herb.!; Bertol. fl. it. IX, p. 538; Parlat. fl. it. III, p. 482; O. militaris \& L. sp. pl. 1334; O. Simia Lamk. fl. franc. III, p. 50\%, var. $\beta$; Ambros. fl. Tir. austr. I, p. b31, var. $\alpha$; Vis. et Succ. cat. p. 52. - Vernacolo: Castagnole, Castagnola. - Icon. : Barla op. cit. tab. 35, fig. 1-13. 
- Spica sub-ovata, post anthesim cilindracea, ovariis rachidi - arcte adpressis, unam partem floribus spectantibus. La- bəlli laciniae purpureae, violaceae, roseas, saepe fere al- bae: eodem modo variant tepala exteriora. Dens qui adest - inter partitiones laciniae mediae labelli quandoque elonga- tur, ita ut lacinia ipsa fere trifida videatur. »

$\beta$ nivea. - - Floribus niveis ; O. tephrosanthos var. $\beta$ Pullin. fl. veron. III, p. 11 et herb.!

Comunissima nei prati, nei pascoli, nei boschetti, dalla pianura e dai colli alla regione montana nella quale direnta più rara. - Cresce a Legnago!, Nogara (Masè), Sanguinetto!, Vigasio! ecc.; nei pascoli di Prebian fra Villafranca e Valeggio! (e A. Massalongo); nel Bosco Mantico! (e Manganotti!), in Valdonega!; nei colli di Montorio!, nel Bosco delle Ferrazze!, nel $M$. Trezzolan! ove è abbondantissima; nella Valle d'Illàsi (Abr. e Caro Massalongo!), nei colli di Grezzana! (e De Bracht, A. Massalongo, Tonini!) nel M. Pastello!; nel colle delle Ungherine!; a Pastrengo (B. Pellegrini!); Costermano; sulle sponde del Benaco e nei colli Benacesi a Peschiera!, Colà!, Lazise (C'lementi), Rocca di Garda (Rigo), Scaveaghe!, T'orri (Rigo) ecc.; alle falde del $M$. Baldo presso Rivole, Brentino! ecc. ecc. La var. $\beta$ è rarissima : è stata raccolta da Pollini presso Valeggio (herb.!), da me nel Monte Trezzolan. - Fiorisce in aprile e maggio: anche in giugno nelle stazioni più elerate. そे

Negli esemplari veronesi appartenenti a questa speciè, ho trovato valiabilissima la forma delle brattee, le quali mi si presentarono ovate, acute, acuminate, ottuse, troncate, smarginate benanco ma sempre brevissime, eguaglianti in lunghezza piccola frazione appena dell' ovario, e qualche volla minime o quasi nulle. -- In esemplari che tengo dalla Spagna le brattee, lungamente acuminate, eguagliano in lunghezza la metà dell'ovario.

101. Ot.chis militaris L. fl. suec. ed. 2, p. 310; All. fl. ped. II, p. 148; Pollin. fl. veron. III, p. 12, excl. syn. Seguieri, et herb.!; Bertol. fl. it. IX, p. 540; Ambros. fl. Tir. austr. 1, p. 680, var. $\alpha$; Vis. et Sacc. cat. p. 54 ; O. militaris L. sp. pl. 1333, excl. var. $\beta . \delta . \varepsilon$; Orchis flore Simiam referens, Segu. cat. pl. p. $\approx 6$ et pl. veron. II, p. 127, excl. syn. (et ex parte?). - Vernacolo: Castagnole. - Icon.: Segu. pl. veron, II, tab. XV, n. IX; Barla op. cit. tab. 36, fig. 1-22.

Comunissima dalla bassa pianura a tutta la regione montana; penetra benanco nella subalpina. Si trova nei prati, nei pascoli, nei boschetti di querce, castagni ecc. - Nella pianura presso Legnago!, Vigasio! ecc.; nell'Agro Veronese a Gruastalla!, Sommacampagna!, tra Villafranca e Va- 
leggio nei pascoli di Prebian! ecc. ; presso Verona nei fossi vicini alla Porta del Palio(Seguier), al Pestrino! (Pollin. herb.!, Manganotti!), S. Michele! ecc.; nei boschi lungo l'Adige!; nel Bosco Mantico!; nei colli e monti confinanti col Vicentino e nelle valli dipendenti; a Monteforte!, nella Valle d' Illàsi nel M. Barbara presso Tregnago (A. Massalongo!) ecc., nel M. Trezzolan!, nel vajo di Squaranto!, nei colli di Montorio!, nella Val Pantena (Tonini!), nei Lessini presso Cerro!, Chiesanova! ecc., nel M. Tondo!, nel M. Comun!, nel M. Tesoro!, S. Anna d'Alfaedo!, MI. Pastello!, Val Policella!. eec., a Pastrengo (B. Pellegrini!), Rivole!, Costermano (Manganotti, Rigo), Rocca di Garda (Rigo), sopra Torri nei prati di Pezzoi (Rigo) ecc.; nel M. Baldo a Brentino!, nei prati della Ferrara!, Pravazar!', Ime! ecc. - Fiorisce nei mesi di aprile e maggio nelle stazioni basse, in giugno nei luoghi elevati. $z$

Ho osservato due forme principali: la prima con il caule alto $40-43 \mathrm{~cm}$. e la spiga cilindracea lunga sino a $22-23 \mathrm{~cm}$; la seconda con il caule più basso e la spiga ovata ed assai più piccola, ha un portamento che ricorda quello della 0 . tridentata.

Sospetto che nella pianta del Seguier, Orchis flore Simiam referens l. c., possano essere compendiate tanto la 0 . tephrosanthos che la O. militaris : ciò mi sarebbe dimostrato dal sinonimo del Colonna riportato dal Seguier Orchis altera Oreades Cercopithecophora Ecphr. pars I, p. 320 (cum icone) che per lo appunto appartiene alla 0 . tephrosanthos, mentre la descrizione e la figura del Seguier sono da riferirsi alla 0 . militaris.

102. Orehis purpurea Huds. $f$. angl. ed. I, p. 334; Parlat. fl. it. III, p. 487; O. militaris L. sp. pl. 1334, var. $\beta$ et $\gamma$; O. fusca Jacq. austr. IV, p. 4; All. fl. ped. II, p. 148; Pollin. viag. p. 110; fl veron. III, p. 13 et herb.!; Fr. Fontana cat. p. 32; Bertol. fl. it. IX, p. 511; Ambros. fl. Tir. austr. I, p. 679; Vis. et Sacc. cat. p. 54; Orchis militaris major Segu. cat. pl. p. 76 ; pl. veron. II, p. 125; Moren. herb. - Vernacolo: Castagnole. - Icon.: Segu. pl. veron 11, tab. XY, n. II; Barla op. cit. tab. 3\%. fig. 1-18.

$\beta$ stenoloba Reich. fil. orchid. p. 31; Parlat. fl. it. III, p. 488 .

$\gamma$ alba; O. fusca $\beta$ flore albo Pollin. fl.veron. III, p. 13; Fr. Fontana cat. p. 32.

Nei luoghi boschivi e nei pascoli specialmente della zona collina $\theta$ montana copiosamente: meno frequente si incontra nella pianura: non la ho mai vista nei luoghi subalpini. - Il Pollini indica questa bella specie, e possiamo dire la massima delle Orchidacee nostrali, alle falde settentrionali del M. Baldo verso Tierno e Castione (300-500 m.), e a Lazise 
ove è pure segnalata dal Fontana, Ospedaletto, S. Ambrogio, Grezzana (e Seguier); A. Massalongo presso Tregnago nel Bosco di Ferrari! (e Da Campo herb. !), e nel M. Precastio!; Gregorio Rigo alla Rocea di Garda, a Passone (m. 347) alle falde meridionali del $M$. Baldo ecc. Io la ho raccolta od osservata nei boschi lungo l'Adigo; presso Guastalla; sui due versanti del Baldo alle falde del monte; nel $M$. Pastello sopra Cavalo e Fumane; nel M. Tondo (m. 640); nei M. Lessini; nel Colle delle Ungherine ed in tutti gli altri colli prossimi a Verona; nel vaio di Squaranto; presso Montorio, Marcellise, alla Mosella (m. 92); nel M. Trezzolan; a Castagnè; presso Monteforte, Soave, Costalunga, Roncà ecc.; nel M. Bolca ecc. La var. $\beta$ è assai rara ed il Parlatore sospetta possa essere una forma ibrida: essa fu trovata dal dottore Facchini nella Valle dell'Adige presso Rovereto; probabilmente potrà benanco rinvenirsi sul suolo veronese. La var. $\gamma$ è rarissima: fu scoperta dal sig. Francesco Fontana presso Lazise sul Benaco. - L'amico Masè ha pure raccolto la 0 . purpurea a Casteldario al confine del Mantovano col Veronese. - Fiorisce da aprile a maggio ed anche in giugno. 2

La spiga è più o meno ricca di fiori, ascendendo questi negli esemplari più lussureggianti sino a $48,50,60$, in altri invece trovandosi anche inferiori a 20 . Gli esemplari frequentemente sembrano poverissimi di fiori per l'arvenuto abortimento di una parte di questi.

\section{Sub-genus 2. - Androrchis Reichb. fil. Orchid. p. 34.}

\section{Sectio 1. - Provinciales.}

103. Orehis provincialis Balb. misc. bot. alt. p. 33 ; Bertol. fl. it. IX, p. 516; Pollin. fl. veron. III, p. 8, in adnotationibus ad Orchidem Morionem; Parlat. fl. it. III, p. 491; Vis. et Sacc. cat. p. 55. - Icon.: Barla op. cit. tab. 38, fig. 1-5.

Il prof. Abramo Massalongo ha scoperto l'O. provincialis nel $M$. Baldo, come risulta dall'esemplare che si conserva nell' Erhario centrale di Firenze: mi è impossibile indicare qualo sia la stazione precisa nella quale il celebre botanjco veroneso rinvenne questa specie. Il direttore del R. Museo e Orto botanico di Firenze, prof. T'eodoro Caruel, interrogato su questo proposito da me, mi scrive che sulla cartolina la quale accompagna l'esemplare sta semplicemente scritto $e$ M. Baldo. Io sino ad oggi ho valiamente cercato sul M. Baldo questa specie, nè la rinvennero il Tonini, il Manganotti, il Rigo. Nel 1875, se non mi tradisce la memoria, ne vidi un esemplare, pur troppo guasto e malandato, nei colli della Valle Pantena sopra le Stelle. Specie da ricercarsi, e che raccomando agli amici e culleghi che percorrono i monti della Provincia.

L'Autore della Flora veronensis evidentemente non vide giammai la O. provincialis: diversamente non avrebbe scritto clie questa bella ed 
ottima specie non solo è prossima alla O. Morio, ma è da ritenersi como una semplice varietà della stessa.

104. Orchis laxiflora Laml. fl. franc. ed. 1, III, 21. 504; Pollin. fl. veron. III, p. 11; Bertol. fl. it. IX, p. 549; Parlat. fl. it. III, p. 496; Vis. et Sace. cat. p. 55; O. ensifolia Vill. pl. du Dauph. II, p. 29; All. auctar. p. 31; Pollin. herb.!; Orchis Morio foemina, calcari extuberante, bifariamque diviso Segu. cat. pl. p. 77 ; pl. veron. II, p. 126; Moren. herb. - Vernacolo: Castagnole. - Icon.: Segu. pl. veron. II, tab. XV, n. VIII; Barla op. cit. tab. 39, fig. 1-20.

$\beta$ bifida. - - Calcare apice dilatato, bifido, lobis late - divaricatis, extrorsum recurvis. Formis intermediis tran- sit in formam genuinam. ,

$\gamma$ brevifolia? Tin. in Guss. syn. fl. sic. IT, p. 535.

Iuoghi acquitrinosi della pianura: più raramente nei colli. Presso Legnago! (e Pollini, Rocchetti), Nogara (Masè), Vigasio! ecc.; nei prati lungo le rive del Mincio a Valeggio (Pollini), Monzambano (Rigo), Salionze (Rigo) ecc.; a Peschiera!; nella Valle dell'Adige sotto Rivole! e lungo il corso del fiume presso Incanale!, la Casetta! ecc.; nei colli Benacesi e in vicinanza delle rive del lago presso Garda (Rigo), alla Volpara! (Rigo), sopra Torri (Rigo) ecc. La var. $\beta$ cresce assieme alla forma tipica; la var. $\gamma$ ? presso Peschiera. - Dalla fine di aprile a tutto giugno. 2

Ho trovato questa specie assai variabile. Esaminatala in diverse stazioni e sopra un grandissimo numero di esemplari, ho verificato, che non sempre, anzi dovrei dire frequentemente, la stessa nei particolari corrispondeva alla descrizione datane dagli autori. Varia la lunghezza della spiga, come il numero dei fori, i quali sono ora più ora meno discosti od avricinati. La lunghezza delle foglie eguaglia frequentemente o su. pera appena il fusto, lasciando libera interamente la spiga. Le brattee, e qualche volta sopra di una stessa pianta, sono ora più lunghe dell'ovario, altra volta uguali ad esso, ovvero più brevi; per lo più quinquenervi. I a rachide che ordinariamente è scabra o scabrosetta negli angoli, si incontra pure affatto liscia. Lo sperone è frequentemente ottuso, altravolta quasi troncato, ovvero leggermente smarginato: ma merita speciale menzione una forma (var. $\beta$ ), che corrisponde forse alla pianta del Seguier superiormente citata; nella quale lo sperone va rapidamente diminuendo di spessore, dilatandosi notevolmente nel tempo stesso, talchè la estremità si presenta affatto piana, con larga smarginatura, i lati della quale incurvandosi dall' interno all'esterno si protendono all' infuori quasi in forma di orecchiette. - Ho raccolto presso Peschierc una forma nana, alta, la spiga compresa, circa 15 centinetri : la spiga, brevissima, si componeva appena di 3-5 fiori. Questa forma potrebbe forse essere rife- 
rita alla varietà brevifolia del Tineo $l$. c.; ma non avendo mai vista la vera pianta del botanico siciliano, non posso asserire nulla in proposito. Probabilmente potrà rinvenirsi presso di noi la $O$. palıstris Jacq.

\section{Sectio 2. - Masculae.}

105. Orehis pallens $L$. mant. alt. p. 292; Allion. fl. ped. II, p. 147; Pollin. fl. veron. 111, p. 15 et herb.!; Bertol. fl. it. IX, p. 515; Ambros. fl. Tir. austr. I, p. 689; Vis. et Sacc. cat. p. 55; Orchis bulbosa, floribus flavescentibus Segu. $p l$. veron. IIT, p. 217; Orchis montana lutea Moren. herb. Icon.: Segı..pl. veron. III, tab. VIII, n. III; Barla op. cit. tab. 43, fig. 1-17.

Fra il confive della Provincia veronese colla vicentina ed il lago di Garda, si incontra qua e là, per lo più gregaria, nei boschi ed anche nei pascoli della zona montana; qualche volta scende nella collina: però non è molto comune. Seguier indica questa specie nella Valle Orcàna presso S. Burtolomeo tedesco (918 m.); il Pollini nello stesso luogo, l'unico esemplare però che si trova nel suo erbario non ha nella scheda la indicazione della località nella quale venne raccolto; il Manganotti la scoprì nel M. Pastello!, ed il Rigo alle falde occidentali del M. Baldo nei boschetti sopra Torri del Benaco nel luogo detto le Sorti, nella Val Volpara, ecc. $(150-200 \mathrm{~m}$.); io la ho raccolta nei M. Lessini presso Velo! (1292 m.), Roverè di Velo (842 m.), nella salita a Chiesanova fra questo pæese e Corbiol! (916 m.) ecc. - Fiorisce da aprile a giugno. 2

Nelle stazioni veronesi nelle quali ho potuto osservare questa bella specie, ho trovato che la stessa si presenta costantemente con caratteri nettamente definiti: i vari esemplari si assomigliano esattamente, solo danno a vedere una qualche diversità nell'altezza del caule, nella spiga più 0 meno fornita di fiori, nelle foglie più o meno larghe ecc.: le quali variazioni sono evidentemente una conseguenza delle condizioni della stazione in cui vive la pianta. Il caule è alto 15-30 cent. ed anche più: le foglie sono ora acute, altre volte ottusissime anzi rigorosamente tondeggianti, e tali differenze si verificano quasi sempre sopra di una stessa pianta.

Il dottore Facchini (in Ambros. fl. Tir. austr. I, p. 834) nota che questa pianta anno frigido 1844, caulem non edidit. Io ho osservato qualche cosa di simile dopo l'inverno rigidissimo 1879-80: tra Chiesanova e Corbiol nei Lessini, in una stazione nella quale la Orchis pallens si trova assai copiosa, pochissimi individui diedero fiori.

106. Orelnis speciosa Host $f$. austr. II, p. 527; O. mascula L. sp. pl. 1333?; All. fl. ped. II, p. 146; Pollin. viag. p. 119; 
fl. veron. III, p. 8 et herb!; Bertol. fl. it. IX, p. 527; Ambros. fl. Tir. austr. I, p. 690; Vis. et Sacc. cat. p. 55. - Vernacolo: Castagnole, Salep. - Icon.: Segu. pl. veron. II, tab. XV, n. V; Barla op. cit. tab. 4i, fig. 1-21.

- Stirps polymorpha: in ditione nostra praeter genuinam, " quae vulgatissima est, sequentes reperiuntur formae; quae - plus minusve frequentes aut rarae cum illa promiscue \& vivunt.

$\beta$ acutiflora Koch syn. fl. germ. et helv. p. 791.

$\gamma$ macrostachya. - Planta elata; spica 25-30 cent. " longa. "

$\delta$ brachystachya. - a Humilior: spica.breviori vel de" pauperata; quandoque floribus aliquantulum minoribus. " $\varepsilon$ maculata. - "Foliis maculatis. - Orchis Morio mas, foliis maculatis Segı. cat. pl.p. $r 7 ;$ pl. veron. II, p. 124.

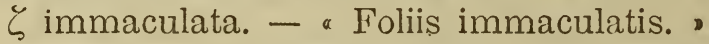

$\eta$ rosea. - "Floribus roseis." - Orchis Morio mas, foliis non maculatis flore roseo Segu. cat. pl. p. $77 ;$ pl. veron. II, p. 125; O. maculata var. b Parlat. fl. it. III, p. 503.

$\theta$ alba. - "Floribus albis. " - Orchis Míorio mas, foliis non maculatis flore candido Moren. herb.; O. maculata var. c Parlat. fl. it. III, p. 503.

Comunissima: dai pascoli elevatissimi del $M$. Baldo, dei Lessini e dei monti confinanti o dipendenti scende ai monti minori, ai colli, ed internandosi nelle valli si avanza nella pianura Veronese nella quale si incontra assai raramente $(2000-15 \mathrm{~m}$.). In tutta la catena del $M$. Baldo tanto sul versante occidentale che sull'orientale; sopra Porcino, a Pozzagalletto, ai Masi, Spiazzi, Pravazar, Basiana, Ime, Valfredda, Naole, Costabella, la Ferrara, Campedello, Lonza, Noveza, Cerbiol, Artillon ecc. ecc.!; nel Pastello! (Manganotti, Rigo); S. Anna, Erbezzo ecc.! ed in tutta la catena dei Lessini!; nel IM. Bolca! ed in tutti i monti che separano il vicentino dal veronese; presso Tregnago ai Finetti, nel $I T$. Belloca ecc. (A. Massalongo!); nel M. Trezzolan!; nel Vajo di Squaranto!, nel Vajo dell'Anguilla e del Falcone!, nella Val Pantena sin presso Grezzana! (e Seguier, Tonini!), al MLaso sopra il Roccolo e nel M. Tondo! ecc. ecc.; e nella bassa pianura presso Nogara (Rodegher) e Isola della Scala! - Tutte le varietà sopra citate crescono promiscuamente: le varietà $\eta$ e $\theta$ le ho di preferenza trovate nei colli alle Volpare ed alle falde del $\boldsymbol{I} \boldsymbol{T}$. Tondo. L'amico Masè ha raccolto la $O$. mascula nel mantovano. - Fiorisce da aprile a giugno secondo la altitudine. $\%$ - Gli erbainoli smerciano gli ingrossamenti tuberiformi delle fibre radicali sotto il nome di Salep, assieme a quelli della 0 . Morio. 
10\%. Orchis Spitzelii Saut. in Koch syn. fl. germ. et helv. ed. 2, p. r90; Reichb. fil. orchid. p. 40, var. a; Bertol. fl. it. IX, p. 525; Ambros. fl. Tir. austr. I, p. 688; Parlat. fl. it. III, p. 508; Vis. et Sacc. cat. p. 55. - Icon.: Reichb. fil. orchid. tab. 383, fig. 1; atlas der alpenflora zu der von prof. Dr. K. W. v. Dalla Torre 439.

Rarissima. Vegeta, dice l'Ambrosi (l. c.) nei luoghi graminosi ed ordinariamente fra le Eriche e la Genista radiata. L' Hausmann la riporta come proveniente dal $M$. Baldo sotto la Colma di Malcesine dove fu trovata dal Leybold (1600 m.). L'illustre botanico, Rev.do Pietro Porta la ha raccolta nello stesso M. Baldo al Trét de spin ed ai Zocchi nei boschi di faggio. - Fiorisce in giugno e luglio. 2

\section{Sectio 3. - Sambucinae.}

108. Orehis sambucina $L . s p . p l$. 1334; All. fl. ped. II, p. 149; Pollin. viag. p. 121 et fl. veron. III, p. 16, var. $x$; Bertol. fl. it. IX, p. 556, var. $\alpha$; Ambros. fl. Tir. austr. I, p. 691; Vis. et Sacc. cat. p. 56, var. a; O. pallens Puccin. fl. luc. $p .476$ non L.; Orchis palmata pratensis latifolia, longis calcaribus, flore albo Segu. cat. $p l . p$. $77 ; p l$. veron. II, p. 132; Orchis palmata, lutea, floris labio maculato Segu. pl. veron. III, p. 219; IIoren. herb. - Icon.: Segu. pl. veron. III, tab. VIII, fig. 5; Barla op. cit. tab. 46, fig. 1-10.

$\beta$ purpurea Koch syn. Al. germ. et helv. ed. 2, p. 792; Vis. et Sacc. cat. p. 56; Orchis incarnata All. fl. ped. II, p. 119 exclus. syn. Seguieri; O. sambusina var. \& Pollin. viag.p. 121, et fl. veron. III, p. 17, exchus. syn. Linn.; O. sambucina var. $\beta$ Bertol. fl. it. IX, p. 55\%; O. sambucina var. b. Parlat. fl. it. 512. - Icon.: Barla op. cit. tab. 46, fig. 11-19.

- Forma genuina duas exhibet variationes, a Clarissimo "Seguiero descriptas: sive var. labello concolore impunctato, - et var. labello lineolis vel maculis violaceis adsperso. Flo" res lutei vel lutescentes: quandoque albidi. - In var. $\beta$ - flores rubri, vel purpurei, vel rubro-violacei. •

S' incontra copiosamente nei prati e nei pascoli dalla regione montana alla alpina; frequentemente nei luoghi scoperti dei boschi di faggio. - Nel M. Baldo, segnalata da tatti i botanici che hanno perlustrato il veronese (Visiani, Tonini!, Manganotti, Ambrosi, Rigo ecc.): Seguier 
la indica a Campedello, Pollini nella Valfredda ed in Naole; la ho trovata io pure in questi luoghi ed inoltre in Ime!, alla Lonza!, Novezina!, Noveza!, Cerbiol! ecc. Cresce pure in tutta la catena dei Lessini p. es. Chiesanuova!, Roverè di Velo!, Velo!, ai Trachi!, Bocca di Selva!, Malèra! ecc.; e nei monti che separano il Veronese dal Vicentino nel M. Alba! (e Seguier) ed altrove. - Le due varietà crescono promiscuamente. - Fiorisce da aprile a giugno. 2

L'Arciprete Masè mi scrive di aver raccolto questa specie nella pianura veronese a $S$. Pietro in Valle: per verità questa mi sembra una stazione poco appropriata alla $O$. sambucina, e sospetto possa trattarsi di una qualche specie diversa.

Il Pollini, $f$. veron. III, $p .17$, ha confuso la $O$. incarnata $L$. con la varietà a fiori rossi della $O$. sambucina: errore, del resto, nel quale sono caduti anche altri botanici.

\section{Sectio 4. - Maculatae.}

109. Orchis maculata $L . s p . p l .1335 ;$ All. fl. ped. II, p. 150; Pollin. viag. p. 1.28; fl. veron. III, p. 18 et herb.!; Bertol. f. it. 1X, p. 555; Ambros. fl. Tir. austr. I, p. 692; Parlat. fl. it. III, p. 516, var. $\alpha$; Vis. et Sacc. cat. p. 56; Orchis palmata montana maculata Segu. cat. pl. p. ry; pl. veron. II, p. 132. - Vernacolo: Erba della man, Zatelle, Nanine, Zattine! - Icon.: Segu. pl.veron. II, tab. XV, n. XVI; Barla op. cit. tab. 47, fig. 1-19.

Frequentissima: dalla zona alpina e subalpina scende nella montana e benanco nella collina, internandosi nelle valli ed avvicinandosi alla pianura; ama i prati, i pascoli, i luoghi boschivi. - Nel $M$. Baldo in tutta la catena: lungo la salita da Brentino al Santuario della Corona! (e Pollini), presso la Ferrara!, Ime!, Val di Borno (Rigo), la Lonza!, Novezina!, Noveza!, Artillon! ecc. ecc.; nel M. Pastello! (e Pollini); nei Lessini presso S. Anna!, Molina!, Erbezzo!, Chiesanuova!, ecc.; nel Vajo del Falcone!, nel Vajo dell'Anguilla! ecc.; presso la Giazza! e Tregnago (A. Massalongo!') ecc.; nel M. Lobbia! ecc. ecc. - Fiorisce da maggio a luglio. 2

In questa specie è variabilissima l'altezza del fusto, il numero delle foglie, la lunghezza delle brattee, la tinta dei fiori. Le foglie, generalmente macchiate, alle volte sono immacolate affatto: nota il Da Campo (herb.!) che maculae nigrae quibus folia in planta vivente sunt notata, exsiccatione evanescunt.

110. Orchis incarnata $L . s p . p l .1335$ et fl. succ. ed. 2, p. 312, excl. syn. Seguieri; Parlat. fl. it. III, p. 520, var. x; O. latifolia Pollin. herb. et fl. veron. III, p. 17 (an ex parte?) excl. syn. 
Seguieri; O. latifolia var. $\beta$ Bertol. fl. it. IX, p. 552; O. comosa var. $\beta$ Ambros. fl. Tir. unstr. I, p. 691 ex parte; O. latifolia $\beta$ incarnata Vis. et Sacc. cat. $p$. 55; Orchis palmata palustris latifolia Moren. herb. - Icon.: Barla op. cit. tab. 50, fig. 1-17.

Nei luoghi palustri e nei prati umidi della pianura veronese: in generale però non molto comune. - Presso Verona al Pestrino (Pollin. herb.!) nelle basse di S. Michele!; presso Campalto!, Giarone!; Belfiore di Porcile! ecc.; a Legnago!, Villa Bartolomea! ecc. - Fiorisce in maggio e giugno. 2

Sono rimasto lungamente titubante nel riferire le forme veronesi alla $O$. incarnata $L$. piuttosto che alla $O$. latifolia $L$. Io ho osservato o raccolto questa specie in diversi punti della pianura veronese; ma $\mathrm{i}$ caratteri di essa, desunti specialmente dalla forma e dalla disposizione delle foglie, o dalla lunghezza delle brattee, sono variabilissimi: e come rettamente osservò l'Ambrosi (osservaz. alla Orchis comosa fl. Tir. austr. I, p. 695), appena servono a caratterizzare degli individui, anzichè delle specie. Mi sono deciso per la $O$. incarnata ossequente al giudizio del Bertoloni, che appunto a questa specie Linneana (corrispondente alla var. $\beta$ della sua $O$. latifolia) attribuì le piante veronesi avute dal Pollini. - Un esemplare raccolto recentemente presso Legnago, potrebbe forse corrispondere alla Orchis Traunsteineri Saut. in Koch syn. fl. germ. et helv. ed. $2, p .793$.

\section{Gen. XXII. - Ophrys.}

\section{Sectio 1. - Araniferae.}

111. Ophrys aranifera Huds. fl. angl. ed. 2, p. 392; Poilin. herb. ex parte!; viag. p. 18, sS et fl. veron. III, p. 26 ex parte; Fi. Fontana cat. p. 32 (ex parte?); Bertol. fl. it. IX, p. 586, var. $\alpha$; Ambros. fl. Tir. austr., I, p. 314; Parlat. fl. it. III, 530; Vis. ct Sacc. cat. p. 5\%; O. fusca Spreng. plant. minus cogn. pug. II, p. 80; Orchis fucum referens flore subvirente Segu. cat. pl. p. $\approx 6 ;$ pl. veron. II, p. 131; Orchis fucum referens major, foliolis superioribus candidis et purpurascentibus Segu. cat. pl. p. $76 ;$ pl. veron. II, p. 130. - Vernacolo: Colombire. - Icon.: Segu. pl. veron. II, tab. XV, n. XIII, et tab. XV, n. XIV (mala); Barla op. cit. tab. 51, fig. 1-9.

È la specie più frequente e comune del genere e forse dell'ordine: essa si incontra in tutta la provincia, raramente solitaria, per lo più gregaria, nei prati, nei pascoli, nei luoghi erbosi, ed anche nei boschetti 
specialmente di querce, tanto nella pianura e nell'agro veronese, come nei colli ed anche nella regione montana spingendosi sino a circa $900 \mathrm{~m}$. di altitudine; avendola raccolta il prof. Abramo Massalongo nel H. Belloca! (830 m.), ed io presso Chiesanuova! - Fiorisce prima fra tutte le Orchidacee veronesi, ed a seconda dell'altitudine da marzo a maggio. 2

E necessario osservare più centinaia di individui appartenenti a questa specie, per avere una idea del polimorfismo di cui la stessa è capace: non solo da un individuo all'altro, ma anche sopra di uno stesso esemplare, i fiori presentano diversità rimarchevolissime, tanto più se prendiamo a considerarli nei diversi stadi di loro vegetazione. - Riferisco alla O. araniferc la pianta del Seguier Orchis fucum referens major ecc. per le figure che l'autore dà delle varie forme che presenta la macchia lucida del labello; !e quali effettivamente si incontrano nella nostra pianta. Il Pollini ha confuso insieme nella sua $O$. aranifera, la vera $O$. aranifera, e la O. Bertolonii Moretti, come risulta dall'esame degli esemplari che si conservano nel suo Erbario, e dal sinonimo O. Speculum Bertol. pl. gen. p. 124 ecc. che spetta alla seconda, e che nella Flora veronensis è attribuito alla prima; e dalla testimonianza dello stesso Bertoloni che scrive aver ricevuta da Pollini la $O$. Bertolonii sub nomine $O$. aranifirae. Non oso asserire se la piauta raccolta da Seguier presso il Castello di Montorio, Orchis fucum referens floribus albis cat. pl. p. 76 e pl. veron. II, p. 131, sia da riferirsi piuttosto alla 0 . aranifera 0 alla 0 . Bertolonii, trattandosi evidentemente di una anamorfosi o se si vuole di un caso di albinismo; stando alla frase descrittiva, attribuisco invece alla seconda la Orchis fucum referens colore rubiginoso che il Bertoloni $I X, p .586$, riporta alla 0 . aranifera.

Malgrado le più diligenti ricerche, sinora non mi venne dato di imbattermi nella forma corrispondente alla $O$. atrata $L i n d l$. : si hanno forme vicinissime a questa, corrispondenti in tutti i caratteri segnatamente per le linee longitudinali, parallele, distinte, glabre del labello; ma nella smarginatura del lobo mediano del labello ho sempre trovato una piccola appendice dentiforme: pero non dubito punto che la 0 . atrata Lindl., se pure è vera e buona specie, possa rinvenirsi presso di noi, come è stata trovata dal chiarissimo Trevisan nei Colli Euganei.

\section{Sectio 2. - Apiferae.}

112. Ophrys apifera Huds. fl. angl., ed. 1, p. 340;. Pollin. viag. 18, 88; fl. veron. III, p. 25 et herb.!; Franc. Fontana cat. p. 32; Reichb. fl. germ. excurs.p. 12s; Bertol. fl. it. IX, p. 582; Ambros. fl. Tir. austr. I, p. 716; Parlat. fl. it. III, p. 538; Vis. et Sacc. cat. p. 57 var. $\alpha$; Orchis araneam referens rostro recurvo Segu. pl. veron. III, p. 246; Moren. herb. - Vernacolo: Colombine. - Icon.: Segu. pl. veron. III, tab. VIII, fig. 2; Barla op. cit., tab. 56. 
Poco frequente, sebbene Pollini scriva che passim occurrit: s' incontra qua e là nei luoghi erbosi dei colli e dei monti minori ed anche, ma più raramente, nella pianura. - In vertice collis qui inter Pogianum et Dominicam vallem medius est (Seguier); nei fossi della città di Verona (Pollini), al Pestrino (De Bracht), S. Pietro in Valle (Masè), presso Grezzana (De Bracht, Tonini!), Avesa (Pollini), Montorio (Pollini), Lazise (Pollini herb.!, Fr. F'ontana), Rocca di Garda (Rigo!), nella Valle dei molini presso Costermano (Rigo): io la ho pure raccolta sulle rive del Benaco a Scaveaghe!, presso Rivole!, lungo l'Adige al piede del monte S. Marco presso la Casetta!, fra la Valle Pantena ed il Vajo di Squaranto nel Monte Sguizzo ecc., ed A. Massalongo nel $M$. Viacara! presso Tregnago $\left(591 \mathrm{~m}\right.$.) e altrove. - Fiorisce dalla fine di aprile a giugno. $\mathcal{\tau}^{\mathrm{a}}$

\section{Sectio 3. - Speculiferae.}

113. Ophrys Bertolonii Morett. pl. it. dec. VI, p. 9; Bertol. f. it. IX, p. 593; Ambros. fl. Tir. austr. I, p. 756 in app.; Parlat. fl. it. III, 513; Vis. et Sacc. cat. p. 57; Goir. app. bot. in cron. alp. p. 79; O. Speculum Bertol. pl. gen. p. 124 et amoen. ital. p. 201; O. aranifera Pullin. fl. veron. III, p. 26 ex parte; Ophrys fucum referens colore rubiginoso Segu. cat. pl. p. 76; pl. veron. III, p. 131. - Vernacolo: Colombine. - Icon.: Barla op. cit., tab. 58, fig. 1-15.

Confusa dal Pollini con la Ophrys aranifera, questa bella specie cresce copiosissima nei prati e nei pascoli della intera regione collina e della montana inferiore: s' incontra pure nell'alta pianura veronese. - Presso Verona a S. Pancrazio!, S. Massimo! (85 m.), in tutte le collinette moreniche della campagna veronese!, in tutti i colli prossimi alla città di Verona!; nella Val Pantena e nei monti circostanti p. e. Zago! (620 m.), Romagnano! ecc.; nei colli di Montorio!; nel M. Trezzolan!; nella Valle d' Illàsi! ecc.; nel M. Pastello presso il fortilizio di Ceraino e altrove!; nell'anfiteatro morenico di Rivole!; alle falde del Monte $S$. Marco e nei luoghi erbosi lungo l'Adige!; nei Colli Benacesi sopra Marciaga, Albisano, Torri ecc. (Rigo). - Dalla metà di aprile ai primi di giugno. 2

\section{Sectio 4. - Tenthrediniferae.}

114. Ophrys Arachnites Host syn. p. 492; Pollin. viag. p. 18, 88; fl. veron. III, p. 27 et herb.!; Fr. Fontana cat. p. 32; Bertol. fl. ital. IX, p. 581; Ambros. fl. Tir. austr. I, p. 715; Parlat. fl. it. III, p. 545, var. $\alpha$; Vis et Sacc. cat. p. 57; Ophrys insectifera $\eta$ arachnites L. sp. pl. 1313; Orchis Arachnites $A l l$. fl. ped. II, p. 147; O. bombyliflora 
Spreng. plant. min. cogn. pug. II, p. 79; Orchis araneam referens Segu. pl. veron. III, p. 244; Moren. herb. - Vernacolo: Colombine. - Icon.: Segu.pl. veron. III, tah. VIII, fig. 1; Barla op. cit. tab. 60, fig. 1-11.

Prati e pascoli della pianura e dei colli: penetra anche nella regione montana. - Il Seguier la dice trovata dal Bordoni nella Valle d' Illàsi, presso Cogòlo; nel qual luogo la rinvenni io pure or sono alcuni anni, come pure più in alto presso Giazza!; Pollini la indica in Valdoneg'a presso Verona, Guastalla, Ospedaletto (herb.!), Novare; Francesco Fontana a Lazise; Gregorio Rigo nei colli di Torri nel luogo chiamato Fradèr, a Marciaga!, Costermano, Salionze, Cisano, Lazise ece.; De Bracht nei colli veronesi: io la ho pure raccolta od osservata nella Valle dell' Adige alle falde del M. Pastello presso Ceraino, tra Villafranca e Valeggio nei prati di Prebian, alle sponde del Benaco a Scaveaghe, alle falde dei Lessini presso Lughezzano e più in alto lungo la strada che conduce a C'hiesamuova! ecc. - Maggio-giugno. 2

\section{Sectio 5. - Musciferae.}

115. Ophrys muscifera Huds. fl. angl. ed. 1, p. 340; Ambros. fl. Tir. austr. I, p. 713; Parlat. fl. it. III, p. 552; Vis. ci Sacc. cat. p. 57; Ophrys insectifera $\alpha$ myodes L. sp. pl. 1343; Ophrys myodes Jacq. ic. rar. I, tab. 184; Pollin. fl. veron. III, p. 25 et herb.!; Bertol. fl. it. IX, p. 5\$1; Ophrys Muscaria All. fl. ped. II, p. 14\%. - Icon.: Barla op. cit. tab. 60, fig. 14-20.

Nei luoghi erbosi e boschivi della regione collina e montana: è pianta piuttosto rara; però si trova ordinariamente isolata o tutto al più in pochi individui, qua e là dalle sponde del Benaco al confine orientale della provincia. - Il Pollini la raccolse nel colle chiamato Montindon, Gregorio Rigo sopra Torri del Benaco! nel luogo chiamato Campitelo, le Sorti ecc., Antonio Manganotti alle falde del M. Baldo presso Brentino!, Abramo Massalongo presso Tregnago!; io ho trovato la Ophrys muscifera nei colli Benacesi presso Albisano!, nei pascoli presso S. Anna d'Alfaedo! (939 m.), lungo la strada che conduce da Avesa al Maso presso Calserega!, al Masetto!, al bosco del Romitorio presso il Maso!, nella Val Pantena! ecc. ecc. - Maggio-giugno. 2

Ringrazio quelle egregie persone, le quali col favorirmi di notizie relative alle Orchiòacee veronesi, mi resero più facile la enumerazione delle specie e forme le quali crescono nella nostra provincia. In modo specialissimo poi i miei ringraziamenti devono essere diretti agli egregi professori T. Caruel, P. A. Saccardo, O. Penzig, A. Manganotti, ed ai miei vecchi amici sig. Gregorio Rigo ed arciprete Francesco Masè per la speciale benevolenza della quale hanno dato prova a mio riguardo. 


\section{Ordo X.}

CYPRIPEDIA CEAE.

Le Cypripediaceae sono rappresentate nella Flora Veronese dal solo Cypripedium Calceolus, l'unica specie di questo ordine la quale viva nella catena delle Alpi Italiane.

\section{Gen. I. - Cypripedium.}

116. Cypripedium Calceolus L. sp. pl. p. 1346, var. $\alpha$; All. fl. ped. 1I, p. 151; Pollin. viag. p. 107; flor. veron. III, p. 39 et herb.!; Bertol. fl. it. IX, p. 639; Ambros. fl. Tir. austr. I, p. 743; Parlat. fl. it. III, p. 565; Vis. et Sacc. cat. p. 59; Damasonio bastardo dei moderni, dal Lusitano con nome di Lonchite dipinto e da altri detto Cosmo Sandalo di Pausonia Pona M. B. p. 239; Calceolus Segu. pl. veron. III, p. 253; Calceolus Marianus Moren. herb. - Icon.: Barla op. cit. tab. 63 ; atlas der alpenflora ecc. $44 \mathrm{~S}$.

Rarissimo nel M. Baldo e nei M. Lessini. - Nel Baldo il Pona indica questa bellissima pianta nella Valle dell'Artillon e nell'altro versante verso Malcesine; il Seguier dice che in silvulis Baldi montis e regione Malcesine qua Benacum spectat reperitur; il Pollini la indica pure nella stazione stessa del Seguier e soggiunge praesertim secus praeruptam, horridamque viam di Ventrar dictam. L' Erbario Polliniano conserva un esemplare di Cypripedium Calceolus raccolto sul Baldo nell'anno 1811. Ai giorni nostri, per quel che io ne so, non era più stato ritrovato, quando recentissimamente (20 gingno 1882) l'amico G. Rigo lo scopriva dal lato occidentale del monte in luoghi erti e scoscesi nel luogo chiamato l' Ortal del Deorsil (1400-1500 m.). - Il Cypripedium Calceolus cresce pure nei M. Lessini: il Prof. A. Massalongo lo scoprì presso Roverè di Velo! Fiorisce in maggio e giugno. 2

\section{Ordo XI.}

IRIDACEAE.

Irideae Parlat. fl. it. III, p. 209.

Il numero delle Iridaceae che crescono nel Veronese, sta a quello che rappresenta la somma totale delle specie italiane appartenenti a questo ordine, nel rapporto approssi- 
mativamente di 1 a 4, 3. Possediamo appena poche specie dei generi Crocus, Gladiolus, Iris, Xiphion, IIermodactylus; mancano i generi Romulea, Gynandriris, Thelysin; ed a rigor di termini alcune specie, come Xiphion foetidissimum Parlat., Xiphion vulgare Parlat. ed Ilermodactylus tuberosus Salisb., non sono indigene nostre ed appena possono dirsi inselvatichite. Una buona parte delle specie di questo ordine abita particolarmente le regioni più meridionali e più orientali dí Europa, e non poche mostrano una decisa predilezione se non per le rive del mare, almeno per le stazioni non molto discoste da questo; pertanto la posizione geografica della Provincia e la distanza relativa della nostra collina e della pianura dalle sponde marine, spiegano la tenuità del contributo portato dalle Iridaceae alla nostra Flora.

Le Iridaceae nostre amano i pascoli, i prati, i seminati, i luoghi boschivi ed erbosi, i siti umidi o paludosi, le stazioni rupestri, i vecchi muri a seconda delle varie specie; lo Xiphion Pseido-Acorus Parlat. non abl)andona mai i fossati della pianura od appena tocca la regione dei colli; il Crocus vernus All. invece non scende mai nella collina, ovvero rarissimamente accenna ad essa, mentre in quella vece si estolle insino alle vette delle nostre alpi, ove lo vediamo talvolta aprire i graziosissimi perigoni a fianco alla neve che comincia a squagliarsi.

\section{Tribus I. - Croceae.}

Gen. I. - Crocus.

117. Crocus biflorus Mill. dict. 4; Bertol. descriz. dei zaffer. ital. n. 4 ; fl. it. I, p. 213; Fr. Fontana cat. p. 29; Reichb. fl. germ. excurs. p. 84; Ambros. fl. Tir. austr. I, p. 669 in app.; Parlat. fl. it. III, p. 219; Vis. et Sacc. cat. p. 53; Crocus vernus Moretti, notiz. sop. div. piant. da agg. alla fl. vicent. in mem. ed osserv. I, p. 245 (planta ex locis demissis tantum); Crocus vernus Pollin. viag. p. 83?; $f$. veron. I, p. 46 non Allion. et $111, p .768$ in app. exclus. syn. fere omnibus et habitatione alpina et montana; Crocus lineatus, bulbo membranaceo Pollin. lett. a Gius. Gauteri in bibliot. ital. XLV, p. 413; Croco Calc. viag. di $M$. 
B. p. 9 (ex loco); Crocus vernus minor albicans Segu. cat. plant. p. 33 ; plant. veron. II, p. 55. - Vernacolo: Castagnole!, Castagnola, Magnugole, Patròni (Monti).

$\beta$ lineatus (Jan) elench. $p .1$; Crocus biflorus var. b Janii Reichb. l. c.

$\gamma$ pusillus (Ten.) cat. h. neap. anno 1813; Crocus biflorus var. c Tenorii Reichb. l. c.

$\delta$ forma monstrosa perigonii limbo septempartito.

Comunissimo nei prati e nei pascoli dal piano ai colli non molto elevati, ed allo sbocco delle valli : non lo ho mai incontrato nella regione montana propriamente detta. - In tutta la pianura e nell'agro veronese!; nei dintorni o nelle vicinanze di Verona, Campo Marzo!, Tomba!, S. Massimo!, Chievo!, Bosco Mantico!, ecc., anzi nella cerchia stessa della città p. e. nel giardino del Collegio degli Angeli!, nei terrapieni e nelle scarpe di tutte le opere di fortificazione e nei fossi che circondano le mura ecc ; colline di Sommacampagna!, Guastalla!, Custoza (Rigo), Valeggio; colli Benacesi presso Lazise (Fr. Fontana), Torri, (Rigo); Costermano (G. Fontana); Pastrengo (B. Pellegrini); nella Valle di Caprino (Calceolari); nella Val Policella!; a Parona!; nella Val Pantena (Tonini); nel Vaio di Squaranto presso Pigozzo! ecc. ecc. La var. $\beta$ è la più frequente; la var. $\gamma$ si trova nei luoghi più magri e secchi, le piante veronesi concordano esattamente con gli esemplari napoletani comunicatimi dai signori Cesati e Pedicino; ho trovato la forma anomala $\delta$ in Campo Marzo presso Verona. - Fiorisce dalla metà di gennaio alla fine di marzo: correndo stagioni invernali molto miti mi è capitato di trovarlo in fioritura sin dalla fine di dicembre. E pianta per lo più gregaria; raramente cresce isolata o solitaria. 2

Il Crocus biflorus è pianta straordicariamente polimorfia. Si trova: (a) colle lacinie interne del perianzio di un color violetto pallido e le esterne bianche o bianco-giallognole, sempre però di una tinta gialla più carica alla base, per modo da simulare quasi una macchia; più raramente le lacinie esterne sono esse pure violacee; - (b) colle lacinie interne bianche, e le esterne bianche e lineate; - (c) colle lacinie esterne bianche o bianco-giallognole lineate solo internamente, in modo però che le linee non souo visibili se non per trasparenza; - (d) col tubo concolore, violaceo, ovvero totalmente bianco senza linee; - (e) col tubo candidissimo, ovvero bianco sfumato di viola, lineato. - Anche il numero delle linee varia, e le lacinie esterne frequentemente sono come chiazzate perchè le righe colorate si dilatano e si ramificano, e le ramificazioni anastomizzandosi si intrecciano fra di loro. Varia infine il Crocus biflorus per il numero dei fiori, per la grandezza del perianzio, per le foglie rette od arcuate. Nel Veronese è comunissima la forma ad un fiore solo.

Il Crocus vernus della $f$. veron. $I, p .46$, omissis synonimis fere omnibus, non è altro che il Crocus biflorus Mill. Il Pollini stesso lo dichiara 
in altri suoi scritti, p. e. nelle Osservazioni agrarie inserite negli Atti della Accademia di Verona: di più nella lettera a Giuseppe Gautieri che trovasi nel vol. XLV della Biblioteca Italiana, dice esplicitamente che il Crocus vernus della sua Flora corrisponde alla pianta avuta dallo Jan e dal Welden sotto il nome di Crocus lineatus, soggiungendo che secondo alcuni botanici è questa specie il Crocus biflorus Mill. ed il Crocus pusillus Ten.: erra però quando dice che è volgarissimo sui monti; perchè il Crocus biflorus è pianta della pianura e della collina esclusivamente.,

118. Crocus vernus $A l l$. $f l . p e d . I, p .84 ;$ Moretti not. sopr. div. piant. da agg. alla fl. vicent. in mem. ed osserv. $I$, p. 245 (planta ex locis editioribus tantum); Pollin. viag. p. 116; Bertol. fl. it. I, p. 206, var. $\beta$; Ambros. fl. Tir. austr. I, p. 618; Parlat. fl. ital. .LII, p. 221, var. a; Vis. et Sacc. cat. $p$. 53; Crocus vernus verus Pollin. fl. veron. III, p. 769 in app.; Crocus vernus, bulbo fibrilloso Pollin. lett. al Gaut. in bibliot. ital. XLV, p. 114; Croco marcio Calc. viag. di M. B. p. 10; Primo Croco verno di Carlo Clusio Pona MI. B. p. 188; Croco verno primo del Clusio Pona M. B. p. 239; Crocus vernus latifolius albus variegatus Segıt cat. plant. p. 32 ; plant. veron. II, p. 51; Crocus vernus latifolius purpureo violaceus Segu. cat. plant. p. 32; plant. veron. II, p. 55. - Vernacolo: I nomi stessi indicati per il Crocus biflorus, e nei M. Lessini Semenarole!. Icon. : comp. fl. ital. tab. XX, fig. 1.

$\beta$ albiflorus (Kit.) in Schult. oestr. fl. 1, p. 101; Hopp. und Hornsch. tagenb. einer reis. nach den Kïste des adriat. meer p. 18\%; Bertol. descr. dei zaff. ital. n. 2; non Pollin. fl. veron. III, p. 769 in app.; Crocus vernus Vis. et Sacc. catal. p. 53, var. $\beta$.

$\gamma$ dianthus. - Bulbis scapos binos gerentibus.

$\delta$ prolifer. - Bulbo ad basim bulbillo florifero praedito.

$\varepsilon$ controversus - Staminibus perianthii laciniis ae"quilongis. Haec ulterius inquirenda."

a Flores occurrunt concolores saturate vel dilute pur- pureo-violacei, saepe albi et ad basim laciniarum violaceo" vittati. Variant praeterea magnitudine perianthii. .

Frequentissimo fra il confine vicentino ed il versante occidentale del M. Baldo, nei pascoli della intera zona alpina e subalpina, ed in quasi 
tutta la regione montana. - In tutto il $M$. Baldo dalle cime più elevate p. e. Costabella (2044 m.), Cima di Valfinestra (2150 m.), Sassetto ecc., scendendo ai prati della Ferrara $(815 \mathrm{~m}$ ), e Fraine $(875 \mathrm{~m}$.), ed avanzandosi verso sud sin quasi a toccare la regione collina alla Papalina e presso Brojeschi (568 m.) ove cresce copiosissimo sotto ai castagni; nei M. Lessini lungo tutta la catena, dalla Sega (1490 m.) e dal Corno d'Acquitio (1543 m.) alla Valle d'Illàsi nei monti sovraincombenti a Giazza e Selva di Progno, e dai pascoli più elevati di Podesteria (1660 m), Castelberto (1765 m.), Sparavér (1784 m.) ecc., sin presso al Cerro $(729$ m.) ed a meno di duecento metri più sotto presso Bocca di Vento nel vaio di Squaranto a pochi chilometri di distanza dal punto che in questa valle rappresenta la stazione più settentrionale del Crocus biflorus!. Si trova pare abbondantissimo nel M. Posta!, Campobrun!, al Passo della Lora!. II. Zeola!, M. Alba! ecc. - La var. $\beta$ cresce promiscuamente alla specie nel $M$. Baldo, nei $M$ Lessini e negli altri monti ora nominati, nei luoghi specialmente abbandonati da poco dalla neve; la var. $\gamma$ a due fiori è meno frequente di quella ad un fior solo, si trova anche essa assieme alla forma tipica; la forma $\delta$ è stata da me trovata nei $M$. Lessini presso ai Trachi; la var. $\varepsilon$ con gli stami lunghi quanto le divisioni del perianzio nelle cime più elevate del $M$. Baldo, e credo che la stessa meriti di essere ulteriormente studiata. - $\mathrm{E}$ in fiore dal mese di marzo a luglio; io però la ho trovata fiorita anche nel mese di agosto: l'epoca della fioritura dipende evidentemente dalla altitudine, dalla abbondanza della neve caduta, e sopratutto nelle stazioni elevate dallo andamento della stagione. 2

Come ho detto, il Crocus vernus descritto da Ciro Pollini nel volume $I$ della sua Flora (a. 1882) è tutt' uno con il Crocus biflorus: però l'autore della Flora veronese confondeva insieme le due specie, come risulta dai sinonimi da lui riportati e dallo stesso suo Erbario!, nel quale le stesse si trovano assieme mescolate. Nella appendice inserita in fine al terzo volume (a. 1824) della Flora veronese, $p$. 768, distingueva tre formo 0 lusus, il Crocus lineatus, il Crocus albiflorus, il Crocus vernus verus: ma durano la confusione $\theta$ la incertezza del chiariss. Autore, perchè al Crocus lineatus è benanco assegnata la stazione dei monti, perchè il Crocus albiflorus di Pollini non è già la vera pianta di questo nome, ma bensì la forma a fiori bianchi o bianco-giallognoli del Crocus biflorus, perchè infine, $p$. 769, è detto che: quoad diversam bulbi formam inter varios Croci verni lusus, et imprimis inter varietatem planitiei et collium (C. lineatus) et montanam vel alpinam flore albo minore (C. vernus recent.); hanc distinctionis notam nequivimus agnoscere. Posteriormente (a. 1826) nella lettera a Giuseppe Gautieri il Pollini si dichiarava poco soddisfatto (Bibliot. ital. XLV, p. 413) di quanto aveva scritto nella Flora intorno ai croci o zafferani veronesi, e finalmente considerava come specificamente distinti il Crocus lineatus (bulbo membranaceo) ed il Crocus vernus (bulbo fibrilloso). Ma errò nuovamente scrivendo del primo che cresce tanto nei monti $\theta$ colli che nel piano veronese, e dicendo del secondo che nasce 
nei prati alpini e subalpini assieme al primo. Te due specie nel nostro paese non crescono mai promiscuamente: il Crocus biflorus non abbandona la regione dei colli; non esce dalla montana il Crocus vernus: i punti estremi ai quali si spingono le due specie, dalla pianura al monte o reciprocamente, determinano due curve irregolarissime, le quali giammai offrono un punto di intersezione. - Giuseppe Moretti nella notizia sopra diverse piante da aggiungersi alla Flora Vicentina (Mem. ed Oss. I, p. 245) confonde esso pure il Crocus biflorus ed il Crocus vernus; come facilmente risulta dalla asserzione che il suo Crocus vermus è una piantina di quelle che vegetano in pianura e sulle più alte montagne.

Diverse specie appartenenti a questo genere vengono coltivate nei giardini veronesi.

\section{Tribus 2. - Gladioleae.}

\section{Gen. II. - Gladiolus.}

119. Gladiolus palustris Gaud. fl. helv. I, p. 97; Ambros. fl. Tir. austr. I, p. 631; Parlat. fl. it. III, p. 25\%; Vis. et Sacc. cat. p. 53; G. triphyllus Bertol. mant. fl. alp. apuan. 2. 5 et fl. it. I, p. 225 non Sibth.; Gladiolus floribus uno versu dispositis Zannich. istor. delle piant. ven. p. 113. Icon.: Zannich. op. cit. tab. 53.

Raro. Questa bella specie è stata scoperta dal già mio allievo Prof. Dottore Rodegher nella bassa pianura veronese nei prati umidi presso Nogara: probabilmente crescerà anche in altri siti. - Fiorisce in maggio e giugno. 2

120. Gladiolus itadiens Lobel icon. stirp. p. 99; Gaud. fl. helv. I, p. 96; Ambros. fl. Tir. austr. I, p. 63r; G. segetum Gawl. in bot. mag. tab. 719; Parlat. fl. it. III, p. 263; Vis. et Sacc. cat. p. 53; G. communis All. fl. ped. I, p. 85; Pollin. viag. p. 85 ; fl. veron. I, p. 47, excl. syn. Ponae; Fr. Fontana cat. p. 29; Bertol. fl. it. I, p. 2.27; Gladiolus floribus uno versu dispositis major et procerior, flore purpureo rubente Segu. cat. plant. p. 4r; plant. veron. II, p. 52; Moren. herb. - Vernacolo: Castagnola salvadega, Spade (Monti), Cortelasso!.

Comunissimo nei seminati di tutta la provincia: si incontra anche nei campi, nei luoghi erbosi, negli argini ecc. Dalla pianura ascende ai colli ed alla regione montana nella quale va diventando man mano più raro: presso Verona!, S. IMartino!, S. Bonifacio!, Legnago!, Nogara 
(Rodegher!), Sanguinetto! Acc., Vigasio! Peschiera! ecc.; nei colli!; nelle valli Pantena!, Policella! ecc.; nell' Agro veronese!; fra le messi a Vestena e Bolca!; nei MI. Lessini e nel MT. Baldo frammezzo al Marzuolo sebbene assai raro!; il Rigo lo segnala negli oliveti di Fradèr presso Torri del Benaco, e nei seminati di Lazise, Pacengo ecc.; il Facchini lo raccolse al confine del Trentino col Veronese al Vò per Borghetto. - E in fiore da aprile a giugno, ed anche in luglio nelle stazioni più elevate. 2

Si coltiva nei giardini il Gladiolus communis $L$., al quale credo debbano riferirsi come sinonimi le piante del Pona (ML. Baldo descritto) Gladiolo p. 138 e nell'indice dell' opera Gladiolo di fior rosso, e Gladiolo di for molto grande e tutto candido ibid. p. 138: infatti il Pona nel luogo ora citato parla evidentemente di piante coltivate.

Tribus 3. - Xiphieae.

$$
\text { Gen. III. - IrIS. }
$$

121. Iris florentina $L . \quad s p . ~ p l . ~ 55$; Bertol. fl. it. I, p. 231; Ambros. fl. Tir. austr. I, p. 666 in app.; Partat. fl. it. III, p. 2r 1; Goir. pl. vasc. nov. vel. min. cogn. cut. crit. cent. $I$, p. 27; Iris germanica flore albo MIonti dizion. bot. veron. p. 124? - Icon.: bot. mag. t. 671 .

Rarissima. Nel maggio del 1870 ho trovato in piena fioritura un individuo unico di questa bellissima specie sopra di un muro a secco, nella collina che sta tra Montorio e Marcellise. - Sospetto che alla Iris florentina sia da riferirsi la Iris germanica flore albo (in vernacolo Gazòi bianchi) riportata da Lorenzo Monti nel suo Dizionario botanico veronese (a. 1817), e forse a quei tempi coltivata nei giardini veronesi.

122. Iris germanica L. sp. pl. $55 ;$ Allion. fl. ped. I, p. 36 ex parte?; Pollin. viag. p. 86; fl. veron. I, p. 48; Bertol. fl. it. I, p. 232; Ambros. fl. Tir. austr. I, p. 610; Parlat. 1 . it. 111, p. 274; Vis. et Sacc. cat. p. 52; Iride volgatissima Pona. M. B. p. 1\%; Iris vulgaris germanica, sive sylvestris Segu. cat. plant. p. 58; plant. veron. II, p. 57; Moren. herb. - Vernacolo: Iris, Ireos, Iride, Riosse, Riossi, Gazòi: le tre ultime denominazioni sono più specialmente destinate ad indicare i rizomi mondati e disseccati che corrono in commercio. - Icon.: bot. mag. tab. 670 .

$\beta$ humilis. - Planta pumila. .

$\gamma$ variegata. - Foliis variegatis. 
Vulgatissima, spontanea o coltivata, in tutta la provincia, dalla pianura al limite superiore della regione montana, avendola io trovata in II. Baldo presso Campedello, Meneghei, Ardiforte! alla altitudine cioè di circa 900-1100 m. - Nella pianura come nei colli, dal confine vicentino alle sponde del Benaco, si trova ovunque presso le case coloniche, nei pascoli, al margine dei campi, nei muri vecchi, nelle rupi e benanco nelle siepi: è abbondantissima presso Verona in Val Donega e nella salita fuori porta S. Giorgio alle Torri!, nel colle delle Ungherine!, presso Avesa!, a Quinzano!, Montorio!, nel M. Trezzolan!, nel M. San Marco! ecc. La var. $\beta$ che riproduce la forma genuina sminuita in tutte le sue parti, e porta frequentemente un solo fiore, cresce nei luoghi aridissimi e soleggiati p. e. nel colle delle Ungherine! (282 m.) e nel M. S. Marco! (449 m.): è una bellissima forma, che merita di venire ulteriormente esaminata. La var. $\gamma$ a foglie variegate di verde e bianco è coltivata nei giardini. - Aprile-giugno. $z$ - La Iris germanica è coltivata su vasta scala nella provincia veronese presso Soave, nella Valle d' Illàsi ecc. per ricavarne i rizomi che sono argomento di un ramo non indifferente di commercio sotto il nome di Radice d'Ireos. Può vedersi l'opuscolo del sig. cav. Stefano De Stefani Produzione e commercio della radice dell' Iride germanica nella provincia di Verona, ed un mio articolo sul Corriere della settimana a. II, n. 19.

123. Iris pallida Lamk. encycl. méth. bot. p. 291; Pollin. fl. veron. I, p. 50 in adnot. ad gen. Iris; Bertol. fl. ital. I, p. 234; Amb*os. fl. Tir. austr. I, p. 639; Vis. et Sacc. cat. p. 52.Vernacolo: I nomi stessi della specie precedente colla quale probabilmente è stata confusa per il passato. Icon. : bot. mag. tab. 685 .

$\beta$ floribus laevissime coerulescentibus. Rigo in litt.

$\gamma$ variegata. - Foliis variegatis. ,

$\delta$ anomala. - a Acaulis, uniflora, pedunculo brevi ebracteato inter folia ensiformia repente. Flos intense coeruleus. 'Rigo in litt.

Questa bella specie, frequentemente coltivata per l'odore gratissimo, che specialmente nelle piante da orto acquistano i fiori, si incontra pure spontanea nella provincia nostra, ma meno frequente della specie precedente colla quale qualche volta è mescolata. Si incontra di preferensa nella parte occidentale della provincia fra la riva destra dell'Adige e le sponde del Benaco: l'amico Rigo mi scrive che cresce copicsa nelle colline aride e pietrose sopra Torri dzl Benaco; io la ho trovata presso Cavajon! ed Affi!, lungo la strada che dal fiumo Adige conduce al paese di Rivole!, in vicinanza al forte di Rivole! ecc. Si trova pure nella città stessa di Verona nel Collegio degli Angeli!, e nel cortile del Teatro filarmonico!; presso Parona!, Pescantina!, ecc. La var. $\beta$ ̀̀ stata scoperta dal Rigo fra Lazise 
c la Bagatta lungo la spiaggia del Benaco; la var. $\gamma$ è coltivata nei giardini; la var. $\delta$ è stata trovata dal Rigo nei luoghi pietrosi della collina di Torri del Benaco! - Aprile-giugno. 2

Sospetto che la Iris florentina inserita dal Pollini fl. veron. I, p. 49 in calce alla Iris germanica col sinonimo Iris illyrica Zannich., sia da riferirsi alla Iris pallida: il quale sospetto è legittimato dalla stazione asseguata a questa pianta, cioè l'Istria.

I rizomi della Iris pallida corrono in commercio mescolati a quelli della specie precedente. - Noto infine che in questa specie è assai variabile la tinta dei fiori.

124. Iris squalens $L . s p . p l$. 56; Bertol. fl. it. $111, p .609$; Ambros. fl. Tir. austr. I, p. 611; Vis. et Sacc. cat. p. 52; Iris sambucina Pullin. viag. p. 128?; fl. veron. I, p. 48 e loco non ex descriptione; herb.!; Vis. et Sacc. cat. p. 52 quoad pl. veron? - Vernacolo: I nomi stessi delle due specie precedenti. - Icon.: bot. mag. 787 ; Reichb. icon. fl. germ. et helv. tab. CCCXXXVI, fig. 763.

Mentre la Iris pallida abbonda nella parte occidentale della provincia veronese, la Iris squalens cresce invece copiosa nella orientale, dal limite superiore della pianura alla regione montana, sola ovvero mescolata alla I. germanica, colla quale viene pure promiscuamente coltivata. Io la ho osservata specialmente presso Soave!, Illàsi!, Tregnago!, Monteforte!, Costalunga!, Brognoligo!, Montecchì!, Roncà! e nel M. Bolca (m. 827): le due ultime stazioni ora nominate suno quelle precisamente indicate da Ciro Pollini per la sua $I$. sambucina. - Anche i rizomi della $I$. squalens con quelli delle due specie precedenti corrono in commercio sotto il nome di Ireos.

Nulla posso dire della $I$. sambucina che il Pollini a $p .127$ del viaggio dice di aver osservata sulla destra dell' Adige lungo la strada che parte dal porto della Peraròla a lato ad un antico castello, che altro non può essere se non Incanale: però in quella zona sino ad oggi io non ho visto che la $I$. gerinanica e la $I$. pallida. Sono poi sicurissimo che la $I$. sambucina della Flora veronese corrisponde alla $I$. squalens, perchè nelle stazioni di Roncà e di $M$. Bolca, dopo minutissime ricerche, ho sempre osservato quest'ultima, e nessuna forma che risponda alla prima, e perchè gli esemplari esistenti nell' Erbario Polliniano sotto il nome di I. sambucina spettano certamente alla $I$. squalens. - Nel Dizionario botanico veronese di Lorenzo Monti (a. 1817), a pag. 125 trovo nominate la Iris pumila? (in vernacolo Cagnòi) e la Iris variegata: non so che dire di queste due piante, probabilmente a quell'epoca coltivate nei giardini.

Indipendentemente dalle specie del genere $I r$ is da me elencate nel presente lavoro, forse potrà rinvenirsene qualche altra ancora. Nelle stazioni aride dei colli che circondano Verona, p. e. nel Colle delle Ungherine, ed 
anche in altri punti, p. e. nel MLonte S. Marco, ho osservato forme interessantissime, che però non ho avuto agio di esaminare nelle successive fasi della loro evoluzione: e non è improbabile possa ritrovarsi o la I. Italica Parlat., ovvero qualche altra forma affine a quella che sotto la denominazione di Iris Cengialti l'Ambrosi descrisse nella sua $f$. Tir. austr. I, p. 643.

Gen. IX. - Xiphox.

\section{Sectio 1. - Psendoxiphion.}

125. Xiphion Pseudo-Acorus Parlat. nuov. gen. $e$ more specie di piante monocot. p. 45; A. ital. III, p. 295; Iris Pseudo-Acorus L. sp. pl. p. 56; All. fl. ped. I, p. 85; Pollin. viag. p. 17; fl. veron. $1, p$. 49; Fr. Fontana cat. p. 30; Bertol. fl. ital. I, p. 2:36; Ambros. fl. Tir. austr. I, p. 614; Vis. et Sacc. cat. p. 5i; Iris palustris lutea Segu. cat. plant. p. 58; plant. veron. II, p. 58. - Vernacolo: Irio zalo, Corezoni. - Icon.: fl. dan. tab. 491.

Frequentissimo in tutti $\mathrm{i}$ fossi dalla pianura alla regione collina. In tutta la pianura; Isola della Scala e al Bussè! (e Seguier), Legnago!, Vigasio! ecc.; presso Verona in Campo Marzo!; Montorio!; Monteforte! ; Roncà! ecc.; presso Mozzecane!; a Valeggio!; nella Valle dell'Adige!; a Castelnuovo!; a Peschiera e lungo le rive del Benaco a Lazise, Bardolino ecc.! (e Rigo). - $\mathrm{E}$ in fiore da aprile a luglio. $z^{2}$

126. Xiphion foetidissimum Parlat. nuovi gen. e specie di piant. monocot. p. 15; Parlat. fl. ital. III, p. 297; Iris foetidissima L. sp. pl. p. 57; Pollin. fl. veron. I, p. 49; Rcichb. fl. germ. excurs. I, p. 82; Bertol. fl. ital. I, p. 238; Ambros. fl. Tir. austr. $I$, p. 667 in app.; Iris foctidissima seu Xyris Segu. catal. plant. p. 57; plant. veron. III, p. 298; MIoren. herb. - Vernacolo: Coral, Corai; tali denominazioni però anzichè alla intera pianta, più propriamente spetterebbero alle cassule rnature, le quali aprendosi mettono in evidenza i semi che, come è noto, sono di un bel color rosso scarlatto. - Icon.: Dodon. pempt. p. 247, fig. inf.

Assai raro: si incontra qua e là nei siti umidi ed ombrosi, nelle siepi, nei luoghi boschivi dei colli. Il Seguier indica questa specie nella Valpantena in sylvula comitum Iustorum presso le Stelle; il Pollini in questo istesso luogo e piesso il Castel S. Felice; Gregorio Rigo nella 
Valle dei Molini presso Costermano; io infine la ho trovata nella Valpantena presso Pojano!, in Val Donega!, lungo la via che da Porta S. Giorgio sale alle Torri! - Dalla fine di maggio a luglio. $2<$ - Siccome le cassule, tuttochè maturissine ed aperte, conservano lungamente i semi, così sono adoperate nell' inverno dalle fioraie per far mazzettini.

Io non credo affatto alla spontaneità di questa Iridacea nella nostra provincia. Da epoca remotissima lo Xiphion foctidissimum era coltivato nei giardini veronesi, e credo che col tempo siasi inselvatichito: e veramente osservo che tutti gli esemplari da me trovati crescevano o in vicinanza a luoghi abitati, ovvero a breve distanza da orti e giardini.

127. Xiphion sibiricum Parlat. fl. it. III, p. 300; Iris sibirica L. sp. pl. p. 37; All. auct. ad fl. ped.p. $6 ;$ Pollin. fl. veron. I, p. 50 in adnot. ad gen. Iris; Ambros. fl. Tir. austr. I, p. 615; Vis. et Sacc. cat. p. 52; Iris angustifolia II Clus. rar. pl. hist. p. 229. - Icon.: Clus. loc. cit.

Raro. Nei prati umidi presso Nogara nella bassa pianura veronese (Rodegher). - È in fiore nei mesi di maggio e giugno. 2

128. Xiphion gramineun Parlat. movi gen. e specie di piant. monocot. p. 45; fl. it. $I I I$, p. 303 ; Iris graminea $L$. sp. pl. 58; All. fl. ped. I, p. 86; Pollin. viag. p. $116 ; f$. veron. I, p. 49 et herb.!; Reichb. fl. germ. excurs. p. 82; Bertol. fl. it. I, p. 24; Ambros. fl. Tir. austr. I, p. 646; $V i s$. et Sacc. cat. p. 52; Iris angustifolia prunum redolens major Segı. plant. veron. I11, p. 230; Moren. herb. - Vernacolo: Iride!, Armellin. - Icon. : bot. mag. tab. 681.

$\beta$ sylvaticum comp. fl. it. p. 171; Goir. plant. vasc. ecc. cent. I, p. 27; Iris sylvatica Balb. cat. stirp. hort. bot. taurin. ann. 1813, p. 14.

Questa bella specie si incontra qua e là nei colli e nei monti fra il Benaco ed il confine vicentino, salendo dal limite superiore della pianura alla regione alpina: in alcune stazioni è copiosissima. Seguier e Pollini la indicano nei colli veronesi a Novaglie, e nella zona alpina in Noveza di M. Baldo; Abramo Massalongo la raccolse nella Valle d' Illàsi a Vico presso Tregnago (herb.!); Gregorio Rigo nei luoghi gramignosi delle colline sopra Torri del Benaco chiamati le Sorti, le Anze, ecc., dalle quali scende verso il lago talchè si incontra presso S. Vigilio! $(75 \mathrm{~m}$.$) : io ho$ raccolto lo Xiphion gramineum presso S. Martino, ove è pure indicato da Seguier, e precisamente alla Mosella! (91 m.); nel bosco delle Ferrazze! (e Pollini!); presso Montorio!'; nei colli di S. Mattia!, S. Leonardo', Avesa! (e Pollini); nel M. Baldo in Basiana e lungo la strada che da questo 
luogo conduce in Ime!, nei jascoli e nelle rupi di Valfredda! ecc.; nel complesso fra $75 \mathrm{~m}$. e $1500-1600 \mathrm{~m}$. di altitudine.

La var. $\beta$ è piuttosto rara e cresce promiscuamente alla forma tipica: io la ho raccolta nei colli veronesi a S. Leonardo!. Nell' Erbario Polliniano se ne conserva un esemplare proveniente dal bosco delle Ferrazze: la scheda porta la scritta $A$ n Iris germanica $W$ ? seguita dalla diagnosi della pianta, in calce alla quale si legge a Seguier non descripta. Altro esemplare di questa varietà, mescolato a quelli appartenenti alla specie, si trova fra le piante di Abramo Massalongo, raccolto da questi presso Tregnago!.

Lo Xiphion gramineum è pianta che varia assai nelle dimensioni del fusto, nella grandezza dei fiori, nella lunghezza e nella larghezz foglie. Negli esemplari veronesi le foglie sono larghe $6-8 \mathrm{~mm}$.: in altri provenienti dall'Austria la larghezza delle foglie non supera i $3 \mathrm{~mm}$. Nella var. $\beta$ la brattea, interamente fogliacea, che accompagna il fiore inferiore, è lunga nella pianta veronese 14 centimetri e supera di 7 centimetri i fiori: ho pero visto esemplari con la brattea inferiore leggermente scariosa ai margini e superante appena i fiori. - Tanto la specie che la varietà sono qualche volta coltivate nei giardini.

\section{Sectio 2. - Euxiphion.}

129. Xiphion vulgare Parlat. movi gen. e muve spec. di piant. monocot. p. 45; fl. it. III, p. 306; Iris variabilis Jacq. collect. $I I, p .32$; Iride bulbosa di vari colori, et di foglia angusta di Carlo Clusio Pona M. B. p. 27. - Icon.: bot. mag. tab. 686 .

" Ho raccolto più fiate questa bellissima specie quasi inselvatichita - negli orti di Costermano al principio di giugno »G. Rigo in lit. Come si vede anche dal sinonimo citato del Pona, questa Iridacea da enoca remotissima veniva coltivata nei giardini veronesi.

\section{Gen. V. - Hermodactylus.}

130. Hermodactylus tuberosus Salisb. hortic. soc. I, p. 304; Parlat. movi gen. e nucve spec. di piant. monocot. p. $45 ; \mathrm{f}$. it. 11I, p. 313; Goir. plant. vasc. nov. ecc. cent. I, p. 27; Iris tuberosa L. sp. pl. 18; Pollin. fl. veron. III, p. 769 in app.; Bertol. fl. it. I, p. 242; Vis. et Sacc. cat. p. 52. - Icon.: Reichb. icon. fl. germ. et helv. tab. CCCXLVIII, fig. "7\%6.

Insel vatichita in alcuni giardini veronesi, e particolarmente nel giardino de'conti Giusti in Veronce!. - Fiorisce da febbraio al principio di aprile. $\%$ 


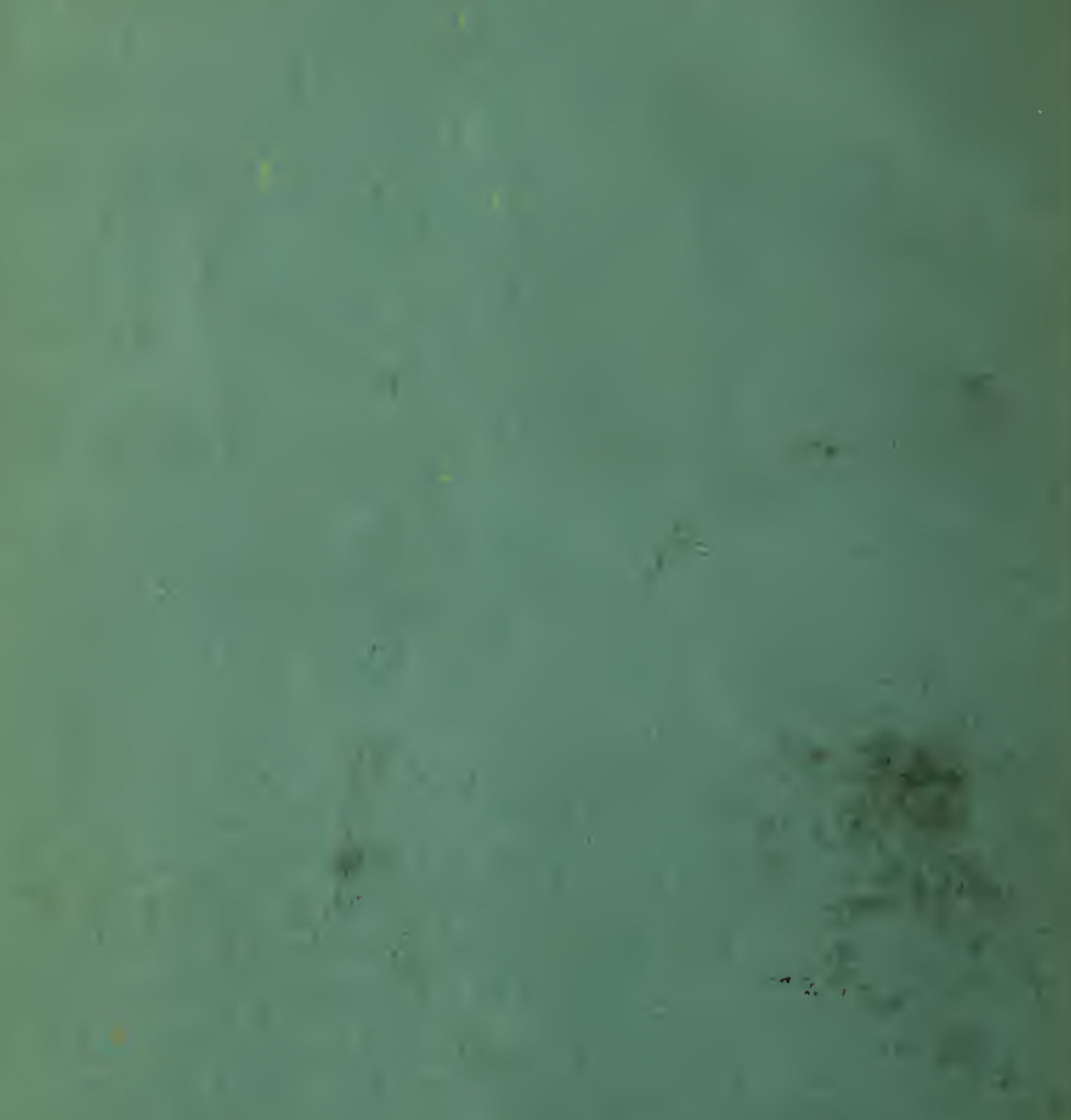

$$
\text { th }-1+2=
$$

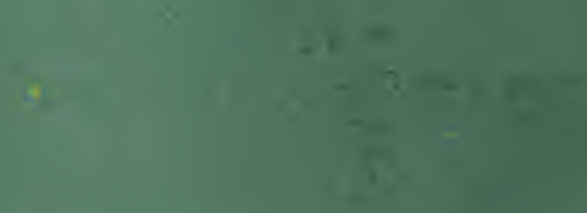


Ql lom

Oroves te Bults fimes s.

a. Goums 


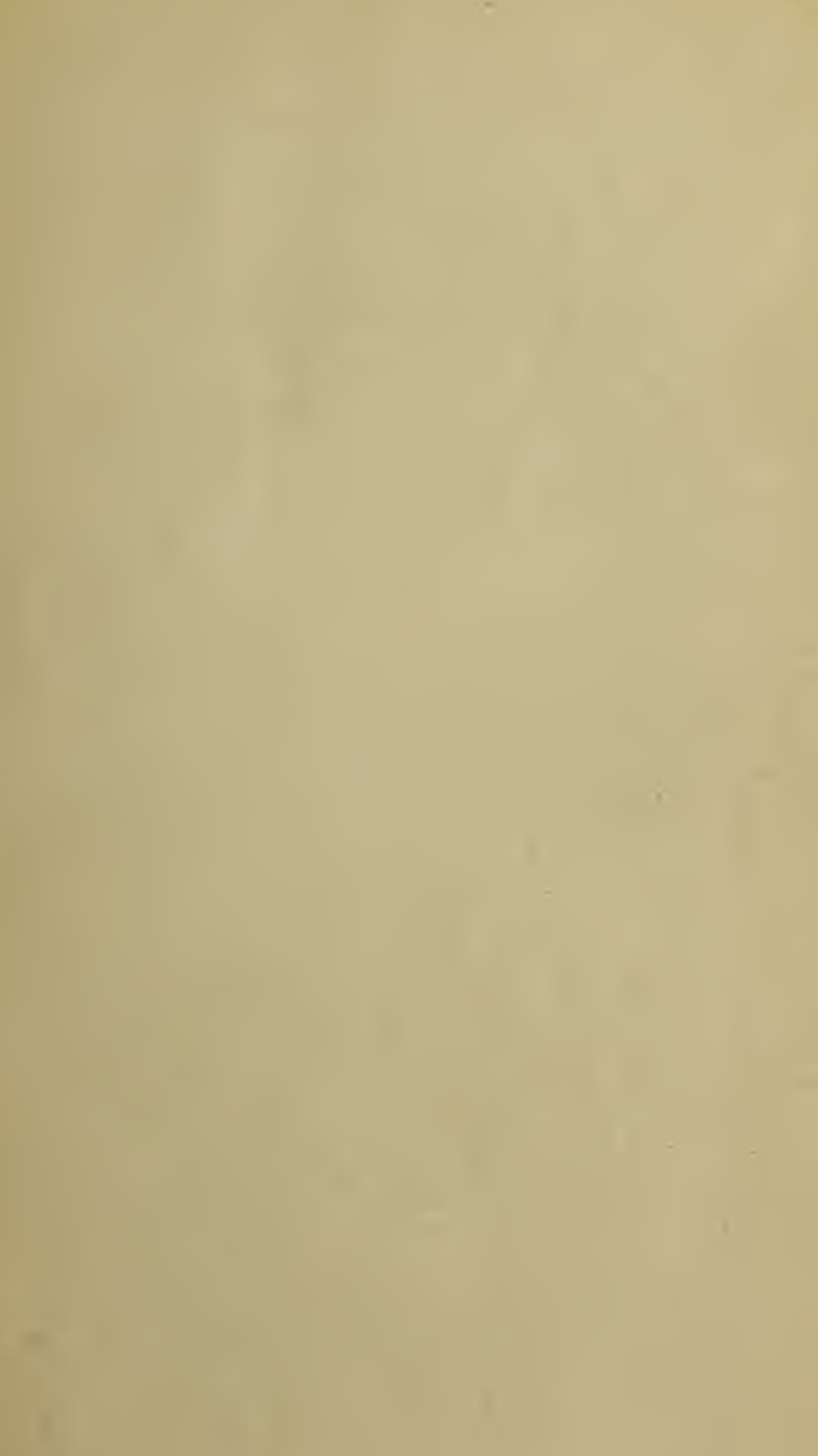

a 



Estratto dal Nuovo Giomale Botanico Italiano, Vol. XVII. N. 1, Gennaio 1885.

PRODROMUS FLORAE VERONENSIS, AUCTORE A. GOIRAN (Continuatio).

Ordo XV.

ASPARAGACEAE.

Le Asparagaceae sono rappresentate nella Flora veronese da tutti i generi crescenti nella penisola e nelle isole nostre, eccezione fatta del genere Myrsiphyllum: questo, come è noto, ha un rappresentante nella Flora italica nel $M$. asparagoides $W$. il quale, oriundo dal Capo di Buona Speranza, vive oramai allo stato salvatico in Sicilia presso Palermo. La nostra zona ricetta pure quasi tutte le specie italiane che compongono i detti generi: ma la esclusione di talune forme nei generi Ruscus, Asparagus, Polygonatum si dimostra evidentemente dipendente dalle condizioni locali e dalla potenza della radiazione solare.

Infatti mentre troviamo presso di noi le forme più settentrionali dell'ordine in Polygonatum officinale All., Majanthemum bifolium Desf., Paris quadrifolia $L$., le specie assenti, se togliamo Polygonatum latifolium Desf., sono precisamente quelle che abitano le arene marittime, o le stazioni prossime al mare, ovvero le regioni più calde d'Italia: e tali sarebbero Aspara- 
gus scaber Brign., A. atbus L., A. aphyllus L., A. stipularis Forsk., Polygonatum Gussonii Parlat.

Le Asparagaceae veronesi sono variamente sparse sul suolo della Provincia: Polygonatum officinale All. dai pressi della città di Verona e dall' Agro veronese si estolle sino a quasi intera la zona subalpina, mentre $P$. multiflorum All. da questa scende nella regione della collina; $P$. verticillatum All., $M I a-$ janthemum bifolium Desf., Streptopus amplexifolius D. C., Paris quadrifolia $L$., salvo rare eccezioni, non escono presso di noi fuori dei confini della regione montana elevata e della subalpina; infine Ruscus aculeatus $L$. e le specie nostrane del genere Asparagus dal piano si estollono appena a tutta la zona dei colli o penetrano tutto al più nella regione montana. Le stazioni infine che le diverse forme prediligono sono subordinate alle speciali loro attitudini od abito: laonde le troviamo raramente nei luoghi coltivati e sabbiosi (A. ofricinalis $L$.), sempre nelle siepi e nelle localita apriche, nei luoghi sassosi o rupestri, negli argini, nelle boscaglie, nei siti umidi ed ombrosi ecc.

Tribus 1. - Asparageae.

Gen. I. - Asparagus.

205. Asparagus tenuifolius Lamk. encycl. I, p. 204; dict. I, p. 274; Pollin. fl. veron. I, $p .456$; Fr. Fontana cat. p. 28 ; Reichb. fl. germ. excurs. I, p. 118; Bertol. fl. it. IV, p. 147 ; Ambros. fl. Tir. austr. I, p. 584; Parlat. fl. it. III, p. 14; Vis. et sacc. cat. p. 50 ; Arcang. comp. del. fl. it. p. 681; Asparagus officinalis var. tenuifolius Pollin. herb.!; Asparagus silvestris, tenuissimo folio Segu. pl. veron. III, p. 90; Moren. herb. - Vernacolo: Sparasina! - Icon.: Red. lil. VIII, tab. 434; comp. fl. it. tab. XVI, fig. 7, b.

« Planta laete viridis: cladodia tenuissima, elongata, ca«pillacea. Variat caule erecto, vel basi prostrato apice adsur" gente. Baccae maximae, semper rubrae, nondum nigrae ut « asserit Cl. Reichenbach. l. c. »

Questa elegantissima specie cresce presso di noi assai copiosa: la troviamo qua e lá, frequontemente gregaria, nella pianura, nell'Agro veronese, nei colli ed anche alle falde dei monti maggiori. Ama i luoghi pietrosi, il margine dei fossati, le siepi, le stazioni 
boschive. - Nell'Agro o nalla pianura veronese presso Valeggio (Pollini), Cadidavid!, al Bovo!, (Buttapietra!), Sanguinetto! (o Seguier, Pollini), l'Allaro (Pollini) ecc. ecc.; alle falde dei Lessini nel Vajo di Squaranto sopra Pigozzo e nei colli circostanti p. e. presso Casale!; nella valle dell'Adige alle falde del M. Pastello sotto al fortilizio di Ceraino!, ed alle falde del M. Baldo sotto al Castello presso Brentino!; nelle vicinanze di Costermano e Garda!; alle sponde del Benaco presso Lazise (Fr. Fontana); ecc. ecs. - Fiorisce in maggio e giugno, qualchevolta sino da aprile secondo l'andamento della stagione: le bacche sono mature in luglio. 20

206. Asparagus officinalis $L . s_{p}$. pl. $p .448$ excl. var. $\beta$; All. fl. ped. II, p. 166; Monti diz. bot. veron. p. 63, 101; Pollin. viag. p. 80 ; Reichb. fl. germ. excurs. p. 118 ; Bertol. fl. it. IT, p. 146; Ambros. Al. Tir. austr. I, p. 582; Parlat. fl. it. III, p. 16; Vis. et Sacc. cat. p. 50 ; Arcang. comp. fl. it. p. 681; Asparagus officinalis $\beta$ altilis Pollin. fl. veron. I, p. 456. - Vernacolo: Sparaso, Spareso, Sparasi, Sparesi. - Icon.: Engl. bot. V, tab. 339.

Si incontra qua e là, per lo più isolato o in piccoli gruppi, nella pianura e nell'Agro veronese; è meno frequente nella regione collina; non ricordo di averlo trovato nella zona montana. Ama i luoghi sabbiosi e boschivi, i campi coltivati, le sponde dei corsi di acqua, gli argini ecc. ecc. - Presso Verona al Pestrino!, in Campo Marzo! ecc.; nei campi, nelle rive e negli argini o nei boschi lungo l'intero corso dell'Adige!; in tutta la pianura presso Arcole!, S. Bonifazio!, Caldiero!, Legnago!, Cerea!, Sanguinetto!, Erbè!, Tarmassia! (e Rigo!), Vigasio! ecc.; nell'Agro veronese presso Villafranca!, Valeggio! ecc.; lungo le sponde del Benaco!; nella valle di Caprino! ( Pollini); presso Rivole ed Incanale nella valle dell'Adige!; nelle valli Policella e Pantena!; nella collina veronese alle Are! ecc. ecc. - Fiorisce in maggio e giugno: le bacche sono mature nell'estate $\theta$ nell'autunno. Recentemente nei luoghi stati invasi dalle acque per la spaventosa piena dell'Adige del 1882 lo ho trovato in piena fioritura nei mesi di settembre ed ottobre. 20

L'Asparagus officinalis è coltivato su vasta scala nella nostra provincia sino da epoca immemorabile: i giovani getti sono portati in mazzi sui nostri mercati sotto il nome di Sparasi. Ai tempi di Seguier ( $p l$. veron. III, $p .9$ ) vendevansi a vilissimo prezzo: in oggi però, causa la sempre cresconte esportazione, mantengonsi a prezzo assai elevato, e sono diventati per i coltivatori sorgente di guadagni non lievi.

Questa specie varia per l'altezza del caule, per la maggiore o minore lunghezza dei cladodi, per la tinta generale della pianta, ora 
di un bel verde, altra volta glaucescente (A. glaucescens Gidgr). La var. a dello Asparagus officinalis Pollin. fl. veron. l. c., come rilevasi benanco dall' Erbario, spetta all'Asparagus scaber Brign., la quale è specie prossima alla nostra però affatto distinta da essa.

207. Asparagus acutifolius $L$. sp. $\mu l . p .419 ; A l l . \wedge . p e d . I I$, p. 167; Monti diz. bot. veron. p. 63, 101; Pollin. viag. p. 14, 80; 1. veron. I, p. 457 ubi excl. loc. agri Tridentini; Fi. Fontana cat. p. 28; Reichb. 1l. germ. excurs. p. 118 ; Bert. fl. it. IV, 1). 151; Ambros. Al. Tir.austr. I, p. 585; Parlat. 1l. it. III, p. 21; ris. et Sacc. cat. p. 50; Goir. erb. forest. veron. p. 16; Arcang. comp. n. it. p. 682; Corruda Ponc M. B. p. 18; Asparagus foliis acutis Segu. cat.pl. p. 13; pl.veron. I, p. 113; Moren. herb. - Vernacolo: Spareselle, Sparaselle, Sparesar salvadego, Sparasini, Sparasina. - Icon.: Corruda prior Clus. ra . pl. hist. II, p. 177; Asparagus foliis acutis Zannich. ist. dell. piant. venet. tab. 179; Sibth. et Smith fl. graec. IV, tab. 337 ; comp. fl. it. tah. XIT, fig. $7, a, c-k$.

«Stirps polymorpha. Tres occurrunt formae precipuae a « longitudine et crassitudine cladodiorum ortae, quae ceterum * formis intermediis confluunt.

$x$ genuinus; A. acutifolius auct. - «Forma typica. Cla« dodiis brevibus: long. 2-6 mm. »

$\beta$ commutatus (Ten.) A. nap. III, p. 374 in obs. ad Asparagum acutifolium. - "Cladodiis crassioribus et duplo longioribus: long. 4-12 mm. »

$\gamma$ ambiguus (De Not.) rep. fl. lig. p. 401 ; Cald. tent. $f$. Favent. in nuov. giom. bot. XII, p. 233. - "Cladodiis tenuis« simis fere capillaceis: long. 4-12 min. Haec saepe sterilis. »

«Variat quoque longitudine caulis: caule, plerumque ra« mosissimo, repente vel inter frutices scandente: cladodiis nunc « atro-virentibus, alias laete viridibus. »

Si incontra copioso nelle stazioni apriche, nei luoghi rupostri e sassosi, nelle siepi, nei boschetti dell'Agro e dell'alta pianura veronese e dei colli: scomparo nella regione montana, o tutto al più appena la tocca. - In Verona nel giardino del Conte Giulio Giusti! e nei fossi stessi della città! (e Seguier, Pollini); presso S. Pancrazio, Tombetta, Tomba, S. Massimo, Chievo! ecc. ecc.; nei colli veronesi!; nella valle dell'Adige alla Chiusa (Rainer in Bertol. l. c.) e 
presso Rivole!; nalla valle di Caprino! (e Pollini) estollendosi sino alle estreme pendici del $M$. Baldo; alle sponde del Benaco presso Lazise, Bardolino, Garda (Pollini, Fontana, Rigo) lungo il Vajo di Squaranto nel quale scompare totalmente non appena oltrepassata la Rocchetta!; nol colle S. Briccio di Lavagno dove cresce copiosissimo alle falde orientali verso la valle di Mezzane!; presso ai Molini di $S$. Michele e da questo punto seguendo sempro la destra dell'Adige insino alla Colombara! ecc. ecs. - Fiorisce in agosto e settembre. - Viene adoperato per infrascar $\mathrm{i}$ bachi da seta: i giovani getti sono nella primavera venduti sui nostri mercati assieme a quelli di Humulus Lupulus sotto il nome di Sparesini, Sparesi salvadeghi, Sparesina. 20

Le due forme $\alpha$ e $\beta$ crescono pər lo più promiscuamente: la $v a r . \gamma$ è stata da me rảccolta in Verona nel giardino Giusti e nei colli di S. Leonardo e S. Mattia. Quest'ultima si distingue a prima vista per i cladodi lunghi, delicati, quasi capillacei: si direbbe quasi costituisca una forma intermediaria tra $A$. acutifolius e $A$. officinalis, o meglio $A$. tenuifolius.

L'A. acutifolius rappresenta nella Flora veronese la specie più meridionale dell'ordine delle Asparagaceae; e l'Agro e la collina veronese danno le coordinate di uno dei punti della linea che segna il limite settentrionale al quale si ferma la vegetazione di questa pianta, che a torto il Pollini indica come vivente nell'Agro Trentino : aff $\lrcorner$ rmando l'Ambrosi 7 . c. che nè il Facchini nè alcun altro botanico potè verificare l'esistenza dell'A. acutifolius nella località dal Pollini riportata.

Tribus 2. - Rusceae.

Gen. II. - RUscus.

208. Ruscus aculeatus L. sp. pl. p). 1474; All. fl. ped. II, p. 221; Monti diz.bot.veron. p. 41, 84, 147; Pollin. viag. p. 18, 87; fl. veron. III, p. 194 et herb.!; Fr. Fontana cat. p. $33 ;$ Bertol. fl. it. X, p. 397; Ambros. fl. Tir. austr. I, p. 621 ; Parlat. fl. it. III, p. 29 ; Vis. et Sacc. cat.p. 51 : Goir. erb. forest. veron. p. 93; Arcang. comp. fl. it. p. 681; Ruscus myrtifolius aculeatus Segu. cat.pl.p. $90 ;$ pl.veron. I, p. 139. - Vemacolo: Brusco, Busassorzi, Bruschiamari, Ponzicul, Spinarati, Bruscanzi, Candalinare, Spin da rati. Icon.: Engl. bot. VIII, tab. 560; comp. fl. it. tab. XVII, fig. 1 .

«Stirps mire polymorpha. In ditione nostra sequentes « legi formas insigniores. 
a genuinus. - « Cladodiis ovatis, ovato-acutis, ovato« acuminatis vel ovato-lanceolatis, in mucronem acutissimum « desinentibus. Forma typica.

"Cladodia conformia in ramis singulis: saepissime e basi « ad apicem ramorum gradatim decrescentia.

$\beta$ stenoclados. - « Planta elatior. valde ramosa; ra* mis divaricato-patentibus, quandoque sed raro arcuatis. Cla« dodiis lanceolatis, approximatis, angustis: long. 27-17 mm., «lat. 7-4 mm.

$\gamma$ loricatus. - « Cladodiis ovato-lanceolatis, acuminatis, « magis contortis, juxta nervum medium plicatis, marginibus « ad apicem conniventibus.

$\delta$ microclados. - « Cladodiis ut in var. $\alpha$ sed brevioribus " angustioribusque: lat. 7-3 mm., long. 15-6 mm.

$\varepsilon$ macroclados. - « Ramis elongatis. Cladodiis distanti« tibus, angustissimis, exquisite lanceolatis, vel lineari-lanceo«latis: lat. 9-3 mm., long. 37-18 mm. Forma elegans et pul« cherrima, sed rara.

$\zeta$ minutissimus. - « Forma pusilla ; tota planta vix 13,5* 17 cent. alta, ramosissima. Ramis erecto-fastigiatis: cladodiis « exiguissimis, dense approximatis.

$n$ Barrelieri; Bruscus seu Ruscus rotundifolius vel Myrtacantha latifolia Barrel. ic. 517; Ruscus aculeatus $\beta$ foliis majoribus Bertol. fl. it. X, p. 397; Parlat. fl. it. III, p. 30."Cladodiis ut in var. a sed latitudine et longitudine longe ma« joribus: lat. $25-17 \mathrm{~mm}$., long. 58-40 mm. »

Frequentissimo nello siepi, lungo gli argini, nei luoghi sassosi e nei boschetti della intera Provincia dal piano a tutta la regione collina fra il Benaco ed il confine vicentino: in qualche luogo, più favorevolmente esposto, accenna benanco a penetrare nella regione montana. - Le var. $\alpha, \beta, \delta$ sono le più comuni e si incontrano ovunque. La var. $\gamma$ singolare per la conformazione dei cladodi i quali sono fortemente contorti alla base, piegati nella nervatura mediana, coi margini conniventi al loro apice - cresce nella collina veronese dalla banda di Valpantena: quivi si rinviene pure, ma rarissima la var. $\varepsilon$, bellissima forma dai rami allungati e dai cladodi distanti, strettissimi, quasi lineari. La var. $\zeta$ riproduce la forma tipica sminuita in tutte le sue parti: cresce nel Bosco Mantico o nel Vajo di Quinzano. La var. $\eta$ infine, riconoscibile immediatamente dalla grandezza dei cladodi, è stata trovata da me nel Bosco Mantico e nel Vajo di Squaranto presso Pigozzo: dessa è importan- 
tissima, come quella che a parer mio rappresenta una forma intermediaria fra il Ruscus acutifolius ed il Ruscus Ilippoglossum.

Fiorisce da gennaio a maggio: peró devo aggiungere che frequentemente lo ho trovato in fiore nell'autunno, durante tutto l'inverno e nella primavera e qualche volta anche in estate, nel corso di tutto l'anno in una parola: le bacche sono mature nell'autunno e si mantengono sino alla primavera dell'anro seguente. 2 - Di questo frutice si mangiano nella prinavera i turioni a guisa di quelli di Asparagus acutifolius, Humulus Lupulus, Clematis Vitalba, Spiraea Aruncus. Lo stesso vale ottimamente a rafforzare le siepi che servono di tutela ai campi, per bordure od ornamento nei giardini, ed a fabbricare granate grossolane per spazzare le vie e stalle. Assieme ad altri frutici è pure adoperato come combustibile.

209. Ruscus Hippoglossum L. sp. pl. p. 1474; All. fl. ped. II, p. 225; Pollin. viag. p. 121; fl. veron. III, p. 195 var. a; herb.!; Reichb. A. germ. excurs. p. 100; Bertol. Al. it. X, p. 399; Ambros. Al. Tir. austr. I, p. 622; Parlat. fl. it. III, p. 32; Vis. et Sacc. cat. p. 51; Goir. erb. forest. veron. p. 93; Arcang. comp. fl.it.p. 681; Hippoglossum Dioscoridis et Lauro Taxa Plinii Column. ecphr. p. 166; Hippoglosso di molti, da Fabio Colonna stimato Radice Idea Pona $M$. B.p. 172; Ruscus angustifolius, fructu folio innascente Segu. cal. pl. p. 90; pl. veron. I, p. 140; Moren. herb. - Vernacolo: Rusco. - Icon.: Sibth. el Smith fl. graec. X, tab. 955; Hypoglosson Laurotaxa Column. ecphr. p. 165.

$\beta$ microglossus (Bertol.) fl. it. $X$, p. 401; Ruscus Hypoglossum var. $\beta$ Pollin. Al. veron. III, p. 195; Ruscus Hypoglossum var. b angustifolius Parlat. fl. it. III, p. 33; Hippoglossi alia species ligulata.... parvis admodum ligulis, dilutiore foliorum colore.... Column. ecphr. p. 167 sub cap. 53.

Questa bella Asparagacea è segnalata dagli antichi botanici veronesi Pona, Seguier, Moreni, Pollini nel M. Baldo fra i sassi della Valfredda: ma nè a me, nè al Rigo fu dato rinvenirla in questa stazione, la quale anco di recente è stata con ogni cura da noi esplorata. Vieno pure coltivata nei giardini in piccoli cespuglietti ovvero come siepe e bordura, ed oggidi cresce abbondantissima ed affatto inselvatichita in Verona nel giardino dell'amico Conte Giulio Giusti. - La var. $\beta$ cresce promiscuamente, o meglio confusa colla specie : dalla quale rigorosamente parlando in molti casi è assai difficile il distinguerla nettamente, p эrchè tanto la maggiore o minore larghezza dei cladodi, quanto le dimensioni della foglia ascellante ecc. 
non offrono che caratteri variabilissimi e di una importanza assai relativa. ${ }^{1}$ - Fiorisce da febbraio ad aprile, $\theta$ anche da dicembre e gennaio se l'inverno è mite. 2

\section{Tribus 3. - Convallarieae.}

Gen. III. - Convallaria.

210. Convallaria majalis $L . s p . p l . p .451$; Monti diz. bot. veron. p. 62, 110; Pollin. viag. p. 112, 125; 1 . veron. I, p. 441 et herb.!; Reichb. 1. germ. excurs. p. 99; Bertol. fl. it. IV, p. 139; Ambros. fl. Tir. austr. I, p. 618; Parlat. fl. it. III, p. 40; Vis. et Sacc. cat.p. 51; Arcang. comp. fl. it. p. 683; Lilio-convallio, ovvero Vernale di Teofrasto, creduto dal Fuchsio Colchico non letale Pona $M . B$. p. 42; Lilio convallio Pona M. B. p. 209; Lilium convallium album Segı. cat. pl. p. 65; pl. veron. I, p. 135; Moren. herb. - Vernacolo: Mughetto, Lili, Liri, Livi, Campanelle, Campanelline. - Icon.: Engl. bot. XV, tab. 1035; comp. fl. it. tab. XVII, fig. 3 .

$\beta$ stenophylla. - « Foliis eximie lanceolatis, angustiori«bus quam in forma typica; lamina foliacea $145 \mathrm{~mm}$. longa, « $20 \mathrm{~mm}$. lata.»

$\gamma$ flore pleno Monti diz. bot.p.62, 110 ; Convallaria majalis $\beta$ flore pleno intus roseo Pollin. $f$. veron $I, p .441$; Lilio convallio con fior rosseggiante Pona M. B. p. 42? - Vernacolo: Lili doppi, Livi doppi.

È pianta gregaria, la quale ama i luoghi salvatici e rupestri, ombrosi o scoperti dei monti, specialmentэ d3lla regione montana elevata; da questa scende talvolta nelle zone inferiori, mentre penetra non di rado nella subalpina: ad ogni modo presso di noi dimostra una speciale predilezione per la zona dei faggi. - Nel $M$. Baldo sul versante occidentale nella valle degli Ossi (Pona, Seguier) e nelle adiacenze di questa (Rigo) tra 1600 e $1800 \mathrm{~m}$. di altitudine, ed alle falde a breve distanza dalle sponde del Benaco ed a meno di $100 \mathrm{~m}$. di altitudine nal luogo detto il Śalto fra Pai e Castelletto!; e sul fianco orientale nella valle dell'Artillon (Pollini), non chè lungo la salita da Brentino a Spiazzi (Pollini), ove si trova a

1 Sulla identità specifica dei tre Ruscus Hypophyllum Linn., R. Hypoglossum Linn., e R. microglossus Bert. Nota di T. Caruel. Nuov. giorn. bot. ital. vol. VIII, p. 60. 
piedi della immensa rupe sovraincombente alla valle del Pissol e sulla quale è costrutto il santuario della Corona!, e più in alto tra il santuario stesso e Spiazzi!; nei Lessini e monti dipendenti p. e. sul M. Pastello (Pollin. herb.!), nei boschi sopra Dolcè, nei monti sopra Peri sotto al Corno d'Aquilio e Rocea pia!, nella salita dal Vò al passo della Segu! ecc.; probabilmente si troverà ancora in altri punti. Il Seguier segnalava ai tempi suoi il Lilium convallium, in omnibus dumetis circa Gratianam vicum: ma in oggi nè da me, nè dal Tonini che minutamente ha visitato quei luoghi, nè da altri per quanto io mi so, vi è stato rinvenuto. Noto che tutte le stazioni surriferite della nostra pianta si trovano distribuite sopra di tina zona, la direzione della quale taglia quasi normalmente la grande vallata dell'Adige. - La var. $\beta$ facilmento riconoscibile alle foglie strette e rigorosamente lanceolate è stata da me fondata su esemplari provenienti dal MI. Pastello e raccolti da Ciro Pollini (herb!): forse ad essa è da riferirsi la var. $b$ minor accennata dal Reichenbach fl. germ. excurs. p. 100. - La var. $\gamma$ è coltivata nei giardini.

Fiorisce in maggio e giugno: però, come mi venne dato di usservare replicatamente, non tutti gli anni. 2 tivato in tutti i nostri giardini per la eleganza e l'odore soavissimo dei fiori: attualmente paro sia per diventare cespite di una fra le numerosissime e piccole industrie alpine. Gli erbaiuoli di montagna infatti cominciano a raccogliere in gran quantita il Mughetto per ismerciarlo poscia ai farmacisti od ai negozianti di medicinali. 亡 noto infatti che questa Asparagacea contiene divorsi principii assai attivi quali la Majalina, l'Acido Majalico ecc. ecc.

\section{Gen. IV. - Polygonatum.}

211. Polygonatum officinale All. fl. ped. I, p. 131; Parlat. fl. it. III, p. 42; Arcang. comp. fl. it. p. 682; Convallaria Polygonatum L. sp. pl. p. 451; Monti diz. bot.p. 82, 110; Pollin. viag. jo. 83, 125; fl. veron. I, p. 442 excl. nonn. syn. herb.!; Franc. Fontana cat. p. 29 ; Bertol. fl. it. $I T$, p. 142; Ambros. fl. Tir. austr. I, p. 615; Polygonatum vulgare Desf. in annal. mus. hist. natur. we Paris IX, $p$. 19; Polygonatum anceps Vis. et Sacc. cat. p. 51. - Vernacolo: Segillo de Salomon. - Icon.: Red. lil. T, tab. 258; Polygogonatum latifolium II, Clus. hist. pl. lib. II, p. 277. $\beta$ flore pleno Pollin. fl. veron. $l$. c.

Frequentissimo nel Veronese, fra il Benaco ed il confine Vicentino, dall'alta pianura $\theta$ dai dintorni di Veronc alla regione subalpina: forma per lo più dei piccoli gruppi, più raramente si trova isolato: oltrepassata la pianura si incontra qualche volta in società con la 
specie seguente. Ama i luoghi sabbiosi nelle stazioni della pianura; i luoghi salvatici, boschivi e rupestri in quelle più elevate. - In Campo Marzo di Verona lungo l'Adige! (e Pollini), alle sponde della isoletta detta del Pestrino! ecc. ecc.; presso le rive del Benaco ai Montioni di Lazise (Fontana, Rigo), nella Busa di Valonè in vicinanza di Torri (Rigo) ecc. ecc.; nella valle di Caprino! (o Pollini); nol $M$. Baldo, nel qualo è copiosissimo tanto sul fianco occidentale cho sull'orientale specialmento in valle Ime!, Basiana!, prosso la $\mathrm{Fer}$ rara!, lungo la ascesa da Brentino alla Corona! (e Pollini); nella valle dell'Adige ed in generale in tutta la catena dei Lessini e monti dipsndenti o finitimi, talchè si trova nel $M$. Pastello, nei monti di Peri, lungo la salita dal Vò alla Sega, a S. Anna d'Alfaedo, Erbezzo ecc., nel Vaio del Falcone, nel Vaio dell'Anguilla, nel M. Tondo, al Maso nel bosco del Romitorio, nella Valpantena, presso Chiesanova, Cerro, Roverè di Velo, Velo, nella valle d' Illàsi ecc. ccc. !. - Fiorisce da aprile a giugno secondo la altitudine. 2 . Nei giardini è qualchevolta coltivata la varietà a fiore doppio.

Prossimo al $P$. officinale All. ̀̀ il P. latifolium Desf. (Convallaria latifolia $J(a c q$.$) , che sino ad oggi in Italia è stato rinvenuto$ soltanto nei boschi della Carnia e dei Monti Vicentini (Vis. et Sacc. cat. p. 51, Arcang. comp. fl. it. p. 682): non è però improbabile cho lo stesso possa rinvenirsi presso di noi e sarebbe da ricercarsi nei Monti Veronesi confinanti con la Provincia Vicentina. - Fra le piante fanerogame del prof. Abramo Massalongo si trova un Polygonatum classificato per $P$. latifolium e raccolto da lui nei $M$. Lessini presso Velo; ma la pianta del Massalongo, pel complesso dei caratteri, mi sembra non spetti punto al $P$. latifolium ma bensi ad una forma latifolia del $P$. multiflorum.

212. Polygonatum multiflorum $A l l$. $A$. ped. I, p. 131; Parlat. fl. it. III, p. 45; Vis. et Sacc. cat.p. 51; Arcang. comp. fl. it. p. 683 ; Convallaria multiflora L. sp.pl.p. 452; Monti diz. bot. veron. p. 82,110 ; Pollin. viag. p. 123; 凡. veron. I, p. 443 et herb.!; Reichb. fl. germ. excurs. p. 100 ; Bertol. fl. il. IV, p. 143 excl. syn. Guss. et Cup. et plant. sic.; Ambros. A. Tir. austr. I, p. 616; Sigillo di Salomone ovver di S. Maria Calc. viag. p. 12; Poligonato di larga foglia Pona M. B.p. 171; Polygonatum latifolium vulgare Zannich. istor. delle piant. venet. p. 235; Segu. cat. pl. p. 83; pl. veron. I, p. 136. - Ternacolo: Segillo de Salomon. - Icon.: Zannich. l. c. tab. 34; engl. bot. IV, tab. 279; Polygonatum latifolium I, Clus. hist. pl. II, p. 215 ; comp. della fl. it. tab. XVII, fig. 4.

\section{«Variat}


$\beta$ bracteatum Parlat. fl. it. III, p. 46; Convallaria multiflora $\beta$ bracteata Koch syn. fl. germ. et helv. edil. 2, p. 814; Ambros. 1 . Tir. austr. I, p. 617.

$\gamma$ pedunculis omnibus bifloris.

* Folia in hac stirpe, forma et magnitudine, summopere va* ria. Occurrunt enim ovata, elliptica, ovato-elliptica, lanceo" lata etc., plus minusve lata et longa, supra viridia et subtus "glaucescentia vel omnino glauca. Praeterea in montibus nos"tris sequentes insigniores formas legi: 1. forma foliis parvis, « viridibus, eximie oratis; 2. forma foliis falcatis: long. $18 \mathrm{~cm}$., "lat. 4-3 cm.; 3. forma foliis lanceolatis, sub-acuminatis, glau" cis, supremis subpetiolatis: long. 7-5 cm., lat. $2-4 \mathrm{~cm}$., fere " intermedia intra $P$. mulliflorum et $P$. verticillatum. - Ul«terius examinandae et inquirendae. »

Meno frequente della specis precedente, colla quale non di rado cresce di conserva: si incontra isolata, ma più frequentemente in piccoli gruppi: abita i luoghi boschivi, salvatici e rupestri della zona subalpina e montana, raramente scende nei colli. - Nei Colli veronesi presso al Maso (461 m.) nel bosco del Romitorio!: nel M. Tondo! (640 m.); nel MI. Baldo presso la Corona!, in Pravazar! (e Pollini), Ime!, Valfredda! (e Pona, Seguier), la Ferrara!, Noveza! (e Seguier), Artillon!, Cerbiol!', Gambon! ecc. ecc.: nei Monti Lessini presso Chiesanuova!, Velo (A. MIass. herb.!) ecc.; nei monti confinanti col Trentino e col Vicentino in Campobrun Zeola (A. Mass. herb.!). - Fiorisce in maggio e giugno : anche in luglio nelle stazioni più elevate. 2

Erroneamente, come già osservó il Reichenbach $l$. c., il Pollini l. c. scrive essere rosse le bacche di questa Asparagacea, le quali invece sono turchine, ovvero appaiono quasi bianchiccie per una sostanza cerea che le ricopre.

Sospetto cho nel suo Polygonatum latifolium vulgare il Seguier possa avere compendiato tanto il $P$. officinale che il $P$. multiflorum, che nelle stazioni indicate dal chiariss. autore ho trovato crescere promiscuamente. Anche Calceolari e Pona secondo tutte le apparenze hanno insieme confuse queste due specie.

Ho raccolto le due varietà $\beta$ e $\gamma$ nel bosco del Romitorio presso il Maso. Segnalo pure ai fitografi ed agli erborizzatori le tre forme da me indicato coi numeri 1,2,3: ho raccolto la prima in Cerbiol di M. Baldo ; la seconda tra Castelletti e la Ferrara in questo stesso monte; la terza noi Lessini. Quest'ultima specialmente è sotto ogni aspetto meritevole di attenzione, come quella che si presenta quasi quale forma intermediaria tra la presante e la specie che segue. 
213. Polygonatum verticillatum All. fl. ped. I, p. 131 ; Parlat. fl. it. III, p.・17; Tis. et Sacc. cat.p. 51; Arcang. comp. fl. it. p. 683; Convallaria verticillata L. sp. pl. p. 4.51; Pollin. viag. p. 95, 107, 112, 125; fl. veron. I, p. $442 \mathrm{et}$ herb.!; Bert. Al. it IV, p. 140; Ambros. A. Tir. austr. I, p. 614; Poligonato dalle foglie strette Calc. viag. p. 14; Poligonato angustifoglio Calc. vicg. p. 10; Poligonato angustifoglio, ovvero minore, stimato dal Mutone Chamedaphne di Dioscoride Pona M. B. p. 171; Polygonatum alpinum non ramosum angustifolium, flore monopetalo albo cylindraceo Martini cat. pl. p. 13; Polygonatum non ramosum angustifolium foliis exadverso ternis, quaternis, vel quinis Ponted. comp. p. 76 ; Segu. cat. pl. p. 83; pl. veron. I. p. 137 et 138. - Icon.: Polygonatum V sive angustifolium I Clus. rar. pl. hist. II, p. 277; Martini M. B. natural. fig. tom. II, p. 17, fig. 13 ined.; Red. lil. V, tab. 244.

Cresce copiosamente qua e là nei luoghi salvatici, rupestri e boschivi della regione montana e subalpina. - Nel $M$. Baldo in Ortigara (Pollini), val Vaccara!, sotto la Bncca di Navene ecc. ecc. sul versante occidentale, o sull'orientale presso la Corona!, in Pravazar! (e Pollin. herb.!), Ime!, Valfredda! (e Calceolari, Pona, Martini), in Noveza!, Acque negre!, Lavacci!, Artillon! ecc. ecc.; $\Theta$ nei Lessini presso Chiesanova!, Trachi!, Roverè di Velo!, Velo! (e A. Mass. herb.!) ecc. ecc. - Fiorisce in maggio e giugno. 2.

Gen. V. - Majanthemui.

214. Majanthemum bifolium Desf. in annal. du mus. d' hist. nat. IX, p. 54; Bertol. fl. it. IT, p. 115; Ambros. fl. Tir. austr. I, p. 619 ; Parlat. fl. it. III, p. 50 ; Vis. et Sacc. cat. p. 51; Arcang. comp. fl.it.p. 683; Convallaria bifolia L. sp. pl. p. 452; Monti diz. bot.p. 70, 110; Pollin.viag.p. 107, 112 ; . veron. I, p. 114 et herb.!; Unifolium quadrifidum All. fl. ped. I, p. 124; Grame di Parnaso Calc. viag. p. 14; Gramegna di Parnasso del Dodoneo Pona M. B. p. 68?; Gramegna di Parnasso, ovvero specie di Lilio convallio presso il Dodoneo, altri la nominó Unifoglio, e il Dalecampio la stimó II specie di Ciclamino presso Dioscoride, altri credè ch'ella fosse la Ceratia di Plinio Pona $M$. B. p. 170 ; Lilium convallium minus Unifolium nemorosum, fiore candido mono- 
petalo quinquefariam diviso Martini cat. pl.p. 12 ; Lilium convallium minus Bifolium nemorosum, flore candido monopetalo quinquefariam diviso Martini l. c.; Unifolium Segu. cat. pl. p. 107 ; pl. veron. I. p. 409. - Vernacolo: Ornio, Orno. - Icon.: Martini M. B. natural. fig. tom. I, p. 49, fig. 41 et p. 50, fig. 52 ined.; Red. lil. IV. tab. 216, fig. 2; comp. fl. it. tab. XVII, fig. 6.

Abita i luoghi umidi, ombrosi $\theta$ rupestri nella regione montana elevata e nella subalpina, specialmente nei boschi di faggio. 亡̇ pianta gregaria. - Nel M. Baldo sul versante occidentale in Ortigara (Rigo), Val Vaccara! (e Calceol.), presso la Bocca di Navene ecc. ecc., e nel fianco orientale in Ime!, Valfredda! (e Pona), Valbrutta!, la Lonza!, Noveza!, Gambon!. Cerbiol!, ai Lavacci!, nei boschi intorno al pian della Cenere!, alla Madonna della neve! (1100 m.), Artillon! (e Rigo), Tredespin! (e Rigo), Zocchi! (e Rigo), ecc. ecc.; nei M. Lessini presso i Trachi!, Revolto! ecc. ecc.; nel M. Zeola!; nel M. Bolca (A. Mass. herb.! 945 m.) ecc. ecc. - Fiorisce in maggio e giugno. 26

Varia questa vaga piantina per l'altezza del fusto e pel numero delle foglie portate da questo. - Non posso affermare se la Gramegna di Parnasso del Dodoneo, che il Pona a p. 68 del suo Viaggio indica come coltivata nei giardini dei Conti Nichesola, sia da riferirsi alla nostra specie, ovvero alla forma crescente nell'America settentrionale e distinta dal Desfontaines col nome di Majanthemum canadense. Del resto per esperienza fatta posso affermare che il $M a$ anthemum bifolium trasportato al piano e coltivato si in vasi che in piena terra fa ottima riescita.

\section{Gen. VI. - StREptopus.}

215. Streptopus amplexifolius $D$. . $f$. fr. III, p. 174; Bertol. fl. it. IV, p. 137 ; Reichb. fl. germ. excurs. p. 100; Ambros. Al. Tir. austr. I, p. 613; Parlat. fl. it. III, p. 52; Vis. et Sacc. cat. p. 51; Arcang. comp. fl. it. p. 683; Uvularia amplexifolia L. sp. pl. p. 436; All. fl. ped. II, p. 160; Pollin. viag. p. 114; l. veron. I, p. 459 et herb.!; Polygonatum latifolium III ramosum Clus. rar. pl. hist. I, p. 276. - Icon.: Clus. l. c.; comp. fl. it. tab. XVII, fig. 5; atlas der alpenflora taf. 450.

Abita i luoghi rupestri e boschivi della regione montana elevata e della subalpina: presso di noi è pianta assai rara. - Nel $M$. Baldo in Noveza sotto alla Coronetta fra le radici di Cytisus alpinus ed i virgulti che sorgono dalle vecchie ceppaie di F'aggio!', ai 
Lavacci e noi dintorni del piano della Cenere! (e Pollin. herb.!, o Rigo), al Tredespin (Rigo); nell'alta valle d'Illasi presso Selva di Progno e nel M. Zeola! (e A. Mass. herb.!). - Fiorisce in maggio $\Theta$ giugno. Le bacche sono mature alla fine di luglio: le stesse sono mangiate dagli uccelli, perchè nel Pian della Cenere mi venne dato di vedere magnifici esemplari di questa Asparagacea sul tronco di un vecchio faggio. $z$ - Il Pollini scrive che in questa specie di spesso le foglie sono segnate da macchie bianche: a me non venne mai dato di verificare tale particolarità, sebbene abbia osservato questa Asparagacea in diversi punti delle Alpi; se pure le macchie viste dal chiariss. Autore non sono dovute alla presenza di un qualche miceto.

\section{Tribus 5. - Parideae.}

\section{Gen. VII. - PARIS.}

216. Paris quadrifolia L. sp. pl. p. 526; All. 1l. ped. I, p. 281 ; Monti dizion. bot. veron. p. 49, 135; Pollin. viag. p. 112 ; fl. veron. I, p. 519; Bertol. Al. it. IV, p. 391 ; Ambros. fl. Tir. austr. I, p. 611; Parlat. fl. it. III, p. 36; Vis. et Sacc. cat. p. 50 ; Arcang. comp. fl. it. p. 683; Herba Paris Calc. viag. p. 12; Herba Paride Pona M. $B$. p. 211; Solanum bacciferum monococcum tetraphyllum luteum, flore viridi et tetrapetalo Martini cat. pl. p. 12; Segu. cat. pl. p. 54; pl. veron. 1, p. 40\%. - Vernacolo: Erba Paris. - Icon.: Martini M. B. natural. fig. tom. I, p. 53, fig. 45 ined.; Red. lil. IV, tab. 226; comp. fl. it. tab. XVII, fig. 2 .

Abita i pascoli ed i luoghi boschivi e ombrosi della regione montana e subalpina; penetra pure nella zona alpina, e qualche volta scende nelle valli: si incontra isolata ovvero in piccoli gruppi. - Cresce copiosa in tutta la catena del $M$. Baldo! dove si trova nella val di Borno (Rigo), in val Vaccara, Naole, Valfredda, Ime, Pravazar, la Ferrara, la Lonza, Novezina, Noveza, Albaré, Gambon, Cerbiol, le Fassole, Artillon, la Prà ecc. ecc. 亡̀ pure frequentissima in tutta la catena dei Lessini!, alla Sega, Podesteria, Trachi, Chiesanova, Revolto ecc. ecc., nonchè nei monti confinanti col Vicentino!, M. Zeola, M. Campostrin, M. Alba ecc. ecc. - Il prof. Abramo Massalongo la raccolse nella valle di Illàsi presso Calavena (herb.!). Fiorisce in giugno e luglio. 2

Il numero delle foglie varia da quattro a sei: le forme però a 5 o 6 foglie presso noi si incontrano assai di rado. Le foglie ordinariamente acuminate all'apice, qualche volta si presentano quasi decisamente ottuse. 


\section{Ordo XVI.}

\section{SMII،ACACEAE.}

La riverenza al nome glorioso di Ciro Pollini mi consiglia a segnare questo Ordine nel presente lavoro. Ma in verità nè a me nè ad altri, almeno per quanto mi consta, venne dato di rinvenire Smilax aspera che l'Autore della Flora veronensis annovera fra le piante nostrane.

\section{Gen. I. - Smilax.}

217. *Smilax aspera L. sp. pl. p. 1158 excl. var. $\beta$; Monti dizion. bot. veron. $p .83,149$; Pollin. fl. veron. III, $p .183$; Bertol. fl. it. $X$, p. 356 ; Parlat. fl. it. III, var. a p. 55; Vis. et Sacc. cat. p. 51; Arcang. comp. fl. it. p. 684. Vernacolo: Smilaze, Smilazze. - Icon.: Sibth. et Smith fl. graec. $X, t a b .959$; comp. fl. it. tab. XVII, fig. 7 ; Smilax aspera rutilo fructu Clus. rar. pl. hist. I, p. 112.

Indicata da Pollini nelle siepi presso Grezzana, dove certamente oggidi non si trova. Credo Smilax aspera debba essere radiata dalla Flora Veronese: e non ritengo improbabile che la pianta vista dal Pollini provenisse da un qualche orto, tanto più che la stessa era adoperata nella farmacia quale succedanea alla Salsapariglia. - Oggidi si trova qualcho volta coltivata presso di noi, quale pianta ornamentale, unitamente ad altre forme congeneri, tanto in vasi, che in piena terra. Nello Square dell' Indipendenza che occupa oggidi l'area dell'antico Orto Botanico, può tuttora vedersi, vestito da lussureggiante vegetazione, un bellissimo campione di Smilax aspera var. mauritanica (Desf.), che da epoca assai remota era coltivato nell' Orto ora ricordato.

\section{Ordo XVII.}

\section{MELANTHACEAE.}

Le Melanthaceae veronesi abbracciano tre generi che complessivamente accolgono cinque forme specifiche: il terzo appena delle specie italiane di questo ordine. La ragione di tale povertà sta nella influenza che la latitudine e la forma che affettano le isotermiche o meglio le isoteriche e le isochimeniche, eserci- 
tano sulla distribuzione geografica delle forme vegetali. Infatti il genere predominante dell'ordine Colchicum, che annovera dieci specie distribuite sul suolo della intera Penisola e delle isole nostre, conta nella nostra Flora due sole specie, in causa appunto della natura meridionale ed orientale di questo genere. - Manca sinora presso di noi il genere Bubbocodium (B. vernum $L$.), indicato del resto in Italia unicamente nei prati del Cenisio e del M. Rosa.

\section{Tribus 1. - Colchiceae. \\ Gen. I. - Colchicum.}

218. Colchicum antumnale $L . s p . p l . p .485 ; A l l . f l . p e d$. I, p. 117; Monti diz. bot. veron. p. 50, 52,60,110; Pollin. viag. p. 83 ; h. veron. $I, p .477$ var. $\alpha$; Fr. Fontana cat.p. 29; Reichb. fl. germ. excurs. p. 96 ; Bertol. fl. it. IV, p. 272; Ambros. fl. Tir. austr. I, p. 523 ; Parlat. fl. it. III, p. 181; Vis. et Sacc. cat. p. 45; Arcang. comp. del. fl. it. p. 707; Colchicum Calc. it. in B. p. 15 (ed. lat.) et Colchico fiorito e Colchico senza fiore Calc. viag. p. 8 (ed. ital.); Hermodattilo o Colchico venenato delle specierie da alcuni stimato Narcisso di Virgilio Pona $M . B . p .143$; Colchicum commune Zannich. ist. delle piant. venet.p. 70 ; Segu. cat. pl. p. 71 et pl. veron. II, p. 54. - Vernacolo: Strangola preti, Castagnole, Famegi, Scartozzi, Grole, Fior per i pioci: (baldenses succo ex floribus et radicibus expresso utuntur ad pediculos enecandos Pollin. fl. veron. I, $p .477$ ). - Icon. : Reichb. ic. fl. germ. et helv. fig. 949-50; comp. fl. it. tab. XIX, fig. 3, o-p.

$\beta$ longiflorum. - «Tepalis lanceolatis obtusiusculis vel sub-acuminatis; long. $52-40 \mathrm{~mm}$., lat. 9-7 mm.

$\gamma$ flore albo Fr. Fontana cat. p. 29.

$\delta$ vernale (Hoffm.) deutsch. fl. ed. 2, tom. I, p. 174; Colchicum autumnale b vernale Parlat. fl. it. III, p. 180. - Icon.: Reichb. icon. fl. germ. et helv. fig. 951.

$\varepsilon$ Forma anomala; folio inferiore transmutato in vaginam $(20 \mathrm{~cm}$. longam in specimine a me lecto) clausam, appendice foliacea brevi instructam. » 
Pianta firequentemente gregaria; più raramente cresce isolata: è comunissima nei prati e pascoli della intera Provincia dalla pianura alla zona alpina, fra il Benaco ed il corso del Mincio, ed il confine Vicentino: si incontra pure n॰i luoghi boschivi. - Fiorisce da agosto ad ottobre: $m i$ è però capitato di trovarlo in fiore anche alla fine di luglio: lo foglie spuntano nell'anno seguente, in marzo nella pianura, nelle stazioni elevate non appena scompare la neve: le cassule maturano da aprile a giugno, ed anche in luglio!, secondo la altitudine. 2

Le cipolle portano da uno a cinque o sei fiori, ed anche un maggior numero nei luoghi pingui: nè tutti ad un tempo, quindi por una stessa pianta la fioritura continua giorni parecchi. La qual cosa fu già scritta dal Bertoloni; il quale osserva inoltre che «qui "viderit plantas in loco natali, obstupesceret lusibus earum. Vix in« dividuum alteri simile l. c. » In tale sovrabbondanza di forme segnalo la mia var. B la quale cresce fuori Porta nuova nei prati del basso Acquar e va distinta per i tepali strettissimi e straordinariamente lunghi. - La tinta dei tepali varia dal roseo o dal violetto roseo al bianco: la forma a fiori bianchi è segnalata da Francesco Fontana presso Lazise: io la ho osservata qualche volta tanto al piano che nel monte. - Nella var. $\gamma$, che frequentemente diventa mostruosa, i fiori spuntano di primavera assieme alle foglie in quelle stazioni che all'autunno si trovano inondate. - Variabili in sommo grado sono la forma e le dimensioni delle foglie: nei boschi che sono tra il Maso e Montechio nella collina veronese, e presso Roverè di Velo nei Lessini ho raccolto esemplari con foglie strettamente lineari; alle falde del $M$. Pastello sopra Fumane altri ne ho osservati con foglie lineari di lunghezza straordinaria; nel colle S. Dionigi sopra Parona ho raccolto una forma con le foglie pure lineari e larghe appena 4 millimetri. $\mathbf{E}$ meritevole di attenzione la forma anomala $\varepsilon$ superiormente segnalata: nella quale la foglia inferiore è convertita in una guaina che avvolge le altre, essendo i suoi lembi congiunti tra loro da una sottile membrana. - Sono convinto che un esamø attento e minuto sui luoghi e sulle piante vive dimostrerà che molte forme le quali corrono sotto il nome di $C$. autumnale appartengono a specie distinte. - Noto ancora che a quanto scrive il Pollini, una forma a fior pieno era ai tempi suoi coltivata nei giardini.

Scrive il diligentissimo Seguier che il Colchicum commune « in « omnibus fere pratis, quae sunt in humili solo reperitur : in illis « etiam oritur, quae parum ab Urbe abscedunt, et a primo Athe« sis flexu comprehenduntur. Segu. pl. veron. l. c. » Stando le cose in tali termini e vista la attuale distribuzione della pianta sul suolo dalla Provincia, sarebbe da conchiudersi che da quei tempi ai giorni nostri, l'area di sua diffusione siasi considerevolmente accresciuta e che la stessa sia andata peregrinando dalla pianura ai monti. Osservo che il Calceolari indica la nostra pianta nell'Agro veronese 
presso Bussolengo, il I'ona nella valle di Caprino: nè l'uno nè l'altro la citano nel Baldo propriamente detto.

219. Colchicum alpinum D. C. $f$. fr. III, p. 195; Gaud. $l$. helv. II, p. 601 ; Reichb. f.germ. excurs. p. 98; Ambros. $\mathrm{f}$. Tir. austr. I, p. 593 in app.; Parlat. A. it. III, p. 184; Vis. et Sacc. cat. p. 45; Arcang. comp. fl. it.p. 707; Colchicum montanum All. Al. ped. I, p. 727 non L.; Colchichum autumnale var. $\beta$ Pollin. l. veron. I, p. 477 excl. syn. Waldst. et Kil., et IVilla.; Colchicum arenarium Reichb. fl. germ. excurs. p. 98 quoad plantam Monzambano, Valeggio, Pavia, Piemonte; Colchicum autumnale * Bertol. fl. it. IV, p. 272 excl. nonn. syn. - Icon.: All. fl. ped. I, tab. 74, fig. 2; Reichb. icon. R. germ. et helv. fig. 946, 947, 948 ; comp. fl. it. tab. XIX, fig. $3, a$.

Rara. Per quante ricerche io abbia fatto, non mi venne mai dato di incontrare questa vaga piantina nè sul $M$. Baldo nè sui Lessini. Il Pollini la indica copiosa lungo il Mincio presso Monzambano e Valeggio: io non ne ho mai fatto ricerca in queste due stazioni; in quella vece la ho raccolta nell'Agro veronese al Bovo a poca distanza da $C a$ di David frammezzo ai ciottoli ivi abbandonati dalle vetustissime alluvioni postglaciali (m. 43). - Fiorisce in agosto e suttembre in società con la Scilla autumnalis $L$. e Spiranthes autumnalis Rich.: spuntano le foglie nell'aprile e maggio dell'anno seguente: non mi fu possibile sino ad oggi veder le capsule. 2

La pianta veronese differisce alquanto da quella dell'Appennino comunicatami dal chiariss. prof. Gibelli, pər essere più piccola di questa in tutte le sue parti; cosi pure da quella delle Alpi valdesi (Rostan), e maggiormente ancora da quelle del M. Rosa (Ab. Carestia).

I sigg. Visiani e Saccardo nel loro eccellente Catalogo non indicano il Colchicum alpinum in alcun punto delle Alpi venete; nè l'Ambrosi lo annovera fra le piante trentine. Reca pertanto meraviglia la presenza di questa pianta al Bovo, nella campagna veronese, confinata sopra una estensione di pochi metri quadrati, ad una altitudine di appena $43 \mathrm{~m}$.; tanto più che la situazione della stazione esclude assolutamente l'ipotesi, che la pianta possa essere stata ivi trasportata e depositata in seguito ad una qualche piena dell'Adige. Essendomi rivolto all'amico Riccardo Avanzi, notissimo illustratore delle formazioni glaciali nel veronese, pэr avere la sua opinione sulla origine e sulla natura del suolo sul quale si trova la stazione della mia pianta, ebbi in risposta da lui una Nota, che credo pregio dell'opera riportare interamente.

« Cà di David sorge sul terre .1o alluvionale del conoide dell'Adige " proveniente, almeno superiormente, dallo sfacelo della morena 
* mediana di Bussolengo. - La giacitura del materiale poligenico " apparisce stratificata senza continuità e con varia pendenza, il che « accennerebbe alla esistenza di correnti limitate e temporanee an«zichè ad una allluvione torrenziale su largo tratto. I ciottoli si « corrispondono per grossezza. Fra essi però trovansi incastrati * dei monoliti piuttosto grossi e deposti conforme le leggi di gra« vità: il che accennerebbe al trasporto su ghiacci galleggianti. Le « stratificazioni sono distinte da straterelli di sabbia e spesse volte " d'argilla interposti. Il materiale diminuendo gradatamente in gros« sezza, sfuma col terriccio vegetale a 4 kilm. nella direzione S. « press'a poco all'altezza di Buttapietra, ad un livello inferiore di $9 \mathrm{~m}$. « da quello di Cà di David. Questi terreni costituiscono il terrazzo "destro dell'Adige, la cui incisione presınta una profondità di circa « $10 \mathrm{~m}$. misurata a S. Giovanni Lupatoto presso a poco all'altezza " di C’à di David. (R. Avanzi). »

La origine glaciale dei terreni sui quali giacciono $C \grave{a}$ di David, il Bovo ecc. darebbe a parer mio una ragione plausibile della presenza del Colchicum alpinum in una stazione la quale sembra poco confacente alla natura di questa pianta.

Se infatti risaliamo all'epoca remotissima nella quale il ritiro dei ghiacciai generava quelle immani correnti cho incisero, rovesciarono e dispersero i depositi morenici, facilmente possiamo immaginare che una flora svariatissima, ben diversa dall'attuale ed in parte almeno alpina, dovette comparire sui greti e nelle sabbie che il ritiro delle acque lasciava allo scoperto. Ma col tempo e col mutarsi dei fattori e delle circostanze esterne mutò pure e scomparve totalmente o quasi la Flora per cosi dire transitoria di quell'epoca, lasciando libero il campo ad altre forme meglio adatte alle nuove condizioni. Alle quali però poterono acconciarsi talune forme forse perchè dotate di maggiore energia di resistenza: talchè riescite vittoriose nella loro lotta per la esistenza, sopravvissero a quelle altre che meno favorite furono condannate a scomparire, perpetuandosi e mantenendosi insino alla epoca nostra. - Il Colchicum alpinum a parer mio sarebbe per lo appunto una di tali forme privilegiate. Nè si credano azzardate tali congetture: nel seguito di questo lavoro non faranno difetto altri casi ed esempi i quali varranno a convalidare queste mie induzioni. ${ }^{1}$

1 Recentemente (15 settembre 1884) ho scoperto una nuova stazione, anche questa limitatissima, di Colchicum alpinum al confine meridionale del Bosco Mantico (89-82 m.) a poco più di un chilometro dalle sponde dell' Adige e all'altezza di circa $10 \mathrm{~m}$. sul letto del fiume. La nuova stazione appartiene precisamente alla stessa formazione geologica nella quale trovasi situata quella del Bovo: e la presenza di $C$. alpinum in questo luogo conferma quanto ho scritto nel testo. 
Tribus 2. - Tofieldieae.

Gen. II. - Tofieldia.

220. Tofieldia calyeulata Wahlenb. tl. lapp. p. 88 in obs. ad Tofieldiam borealem; Gaud. fl. helv. II, p. 594; Reichb. fl. germ. excurs. p. 97; Bertol. $九$. it. IV, p. 24; Ambros. fl. Tir. austr. I, p. 516 ; Parlat. fl. it. III, p. 200; Vis. et Sacc. cat. p. 44; Arcang. comp. fl. it.p. 708; Anthericum calyculatum L. sp. pl. p. 447 ex parte; Narthecium calyculatum $A l l . f l$. ped. II, $p$. 165; Tofieldia palustris Monti diz. bot. veron. p. 85, 152; Pollin. viag. p. 98, 117, 122; fl. veron. I, p. 478 excl. nonn. syn; Falso Asfodello Ongarico del Clusio Pona M. B. p. 174; Asfodello falso del Clusio Pona ibid. p. 241; Phalangium alpinum palustre Iridis folio Segu. cat.pl.p. 81; pl. veron. II, p.61. - Vernacolo: Tajetta, Tajola. - Icon.: Ces. comp. dell. $\mathrm{fl}$. it. tab. XIX, fig. 5 .

a spicaeformis Ambros. 1. Tir. austr. I, p. 517. - Icon.: Segu. pl. veron. II, tab. XIV; Reichb. icon. fl. germ. et helv. fig. 930, 931 .

$\beta$ pygmaea Ambros. l. c.; Tofieldia glacialis Gaur. fl. helv. II, p. 596; Reichb. fl. germ. excurs. I, p. 97; Tofieldia palustris $\gamma$ capitata Hopp. in. herb. lucoeano; Tofieldia calyculata b glacialis Parlat. III. p. 201; Tofieldia calyculata $\beta$ capitata Vis. et Sacc. cat. p. 44. - Icon: Reichb. icon. Al. germ. et helv. fig. 933.

$\gamma$ ramosa Thom. $p l$ exsicc.; Ambros. fl. Tir. austr. I, p. 517; Parlat. fl. it. III, p. 201; Tofieldia glacialis $\beta$ ramosa Gaud. fl. helv. II, p. 526.

«Varietates $\beta$ et $\gamma$ occurrunt etiam tepalis et capsulis rubentibus. »

Cresce nei luoghi umidi, nei pascoli, nelle boscaglie, nei siti pietrosi: abita principalmente lo stazioni comprese nella zona montana superiore, nella subalpina e nella alpina, ma qualche volta scende anche in stazioni più basse avendola io raccolta nella valle dell'Adige al disotto di Rivole, a meno di 190 met.! - Nel M. Baldo è segnalata da Pona, Seguier, Moreni, Pollini, Barbieri, Manganotti, A. Massalongo (herb.!): io la ho raccolta su tutte le creste più ele- 
vate da Costabella all' Altissimo di Nago!, nelle giare di Valbrutta sopra la Ferrara!, allo Struzhenall!, I3asiana!, Valfredda!, Noveza!, Artillon! ecc. ed in tutte le valli che solcano tanto il versante orientale che l'occidentale della catena!. Cresce pure nei $M$. Lessini p. e. presso Revolto!, e negli altri monti finitimi p. e. nel M. Posta!, Campobrun! ecc. ecc. Le due forme $\alpha$ e $\beta$ crescono di solito promiscuamente, ma la $\beta$ ama di preferenza le stazioni più elevate ed alpestri: più rara è la var. $\gamma$; io la ho osservata nel $M$. Baldo nella valle delle pietre. - Fiorisce da luglio a settembre ed ottobre: nelle stazioni basse la ho vista fiorita anche negli ultimi giorni di giugno. 2

\section{Tribus 3. - Veratreae.}

\section{Gen. III. - Veratrum.}

221. Veratrum album $L . s p . p l . p .1479 ;$ Allion. $f$. ped. II, p. 166; Pollin. fl. veron. III, p. 219 var. $\alpha$; Reichb. fl. germ. excurs. p. 97; Ambros. fl. Tir. austr. I, p. 519; Parlat. fl. it. III, p. 204 var. $\alpha$; Arcang. comp. fl. it. p. 708. $\beta$ Lobelianum Pollin. $f$. veron. III, $p$. 220; Koch syn. fl. germ. et helv. edit. 2, p. 831; Parlat. fl. it. III, p. 205; Arcang. comp. dell. fl. it. p. 709; Veratrum Lobelianum Bernh. in Tromms. journ. XVI, p. 206; Reichb. icon. Al. germ. et helv. p. 97 ; Bertol. fl. it. X, 403; Vis. et Sacc. cat.p. 45; Veratrum album Monti diz. bot. veron. p. 50, 53, 79, 153 ; Pollin. viagg. p. 101, 106; Elleboro bianco Calc. viag. p. 12; Helleboro bianco con fior pallido Pona M. B. p. 169; Veratrum flore subviridi Segu. cat. pl. p. 104; pl. veron. I, p. 506 . - Vernacolo: Falagro, Giavardo, Sabadilia nostrana, Sabadilion. Col nome di Giavardo in alcuni luoghi del veronese si indica pure la Scrophularia nodosa L. - Icon.: Reichb. icon. fl. germ. et helv. fig. 937, 938 .

La forma tipica si riconosce immediatamente all'aspetto generale della pianta ed alla tinta dei tepali bianchi di sopra, verdi o verdognoli di sotto. Si incontra rarissimamente, almeno nella nostra zona, in unione alla var. $\beta$ : io la ho sino ad oggi rinvenuta in due sole stazioni, in $M$. Baldo nella Valfredda!, e nei $M$. Lessini ai Trachi!. - La var. $\beta$ è volgatissima $\Theta$ copiosissima in tutta la zona montana e subalpina dei nostri monti, nei pascoli, nelle boscaglie ed anche nei luoghi rupostri, scende qualche volta a stazioni più basse nelle vallate, e non di rado si spinge pure alla zona alpina: si incontra raramente isolata, ma per lo più cresce gregaria formando delle vaste macchie nelle quali fa pompa di una splendida 
- lussuregoriante vegetazione, oltrepassando frequentemente l'altezza di un metro. - In tutta la catena del $M$. Baldo sui due versanti nei quali si innalza sino a qusi $2000 \mathrm{~m}$.!, diventa però più rara verso la sua estremità meridionale: cosi pure cresce in tutto il gruppo dei Lessini e monti dipendenti! - Fiorisce in giugno e luglio. 2 I montanari scavano il rizoma del Falagro e mondatolo diligentemønte lo lasciano, tagliato a pзzzi, seccare al sole : cosi preparato lo vendono ai negozianti di medicinali e di piante industriali.

222. Veratrum nigrum L. sp.pl.p. 1479; Pollin.viag.p. 101, 106, 112; fl. veron. III, p. 221 ; Reichb. fl.germ. excurs. p. 97 ; Ambros. f. Tir. austr. I, p. 521 ; Bertol. fl. it. X, p. 403; Parlat. fl. il. III, p. 207; Vis. et Sacc. cat. p. 44; Arcang. comp. ft. it. p. 703 ; Helleboro bianco con fiori che nel porporeo nereggiano Pona M. B. p. 170; Helleborus albus latissimo nervoso folio, flore imperfecto atrorubente Martin. cat. pl. p. 15; Veratrum flore atro-rubente Segu. cat. pl. p. 104; pl. veron. I, p. 507. - Vernacolo: Falagro. - Icon.: Helleborum album floribus atrorubentibus praecox Lobel. p. 511; Martini M. B. natur. figur. II, tab. 37, fig. 33 ined.; Reichb. icon. fl.germ. et helv. fig. 939; comp. della fl. it. tab. XIX, fig. 6.

Raro: nogli stessi luoghi del Veratrum Lobelianum, ma più di esso dimostra una particolare tendənzi a scendere in istazioni più bass. - 亡 indicato nel M. Baldo da Pona, Martini e Pollini, dai quali è səgnalato in Valfredda, val Vaccara, Valle delle pietre, Artillon, Tredespin, Zocchi ecc. Io lo ho raccolto nella Valfredda! nella stessa stazione notata da Pona, De Bracht, Manganotti ed in Noveza! sotto alla Coronetta. Il Seguier lo indica nei pascoli dei M. Lessini: io lo ho osservato alle falde di questi monti nell'alta Valpantena presso Lugo e Bellori, e più a basso nella valle stessa sul $M$. Tondo (640 m.). - Fiorisce in giugno, luglio ed agosto, ma non tutti gli anni! 2 


\section{.}





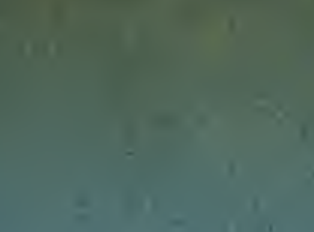

\section{$x \rightarrow$}

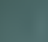

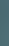

7. $=8$ ,

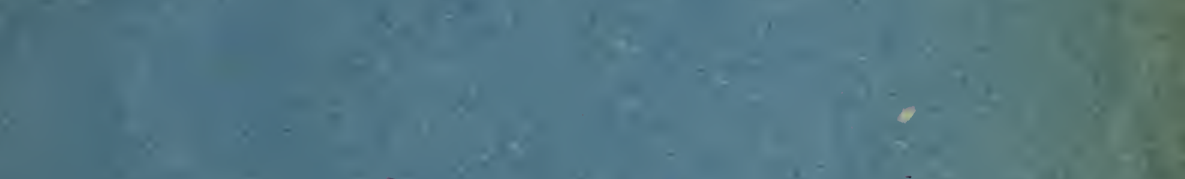

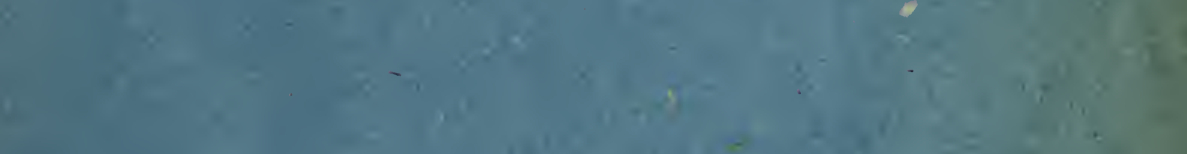
.

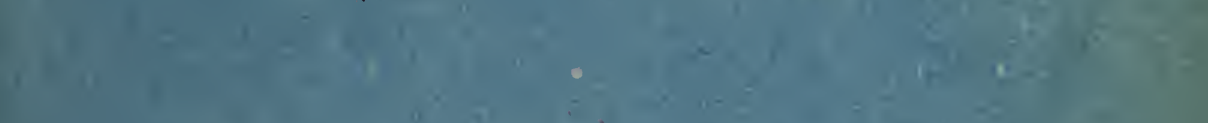

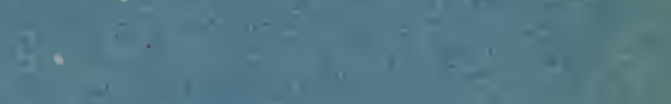
.

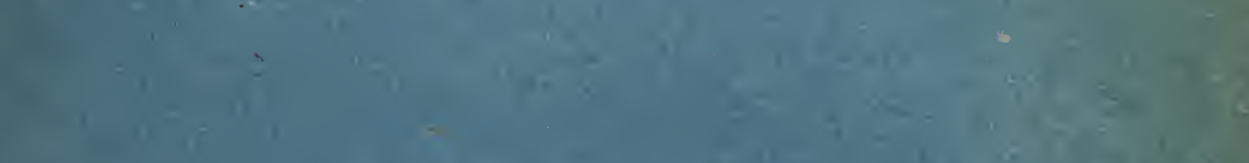

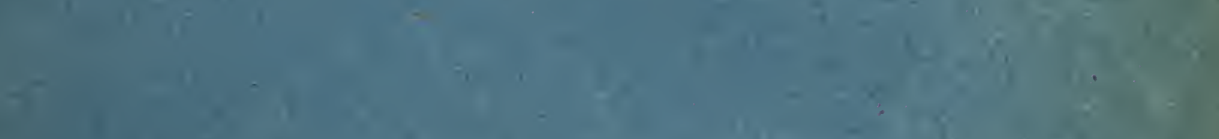
6

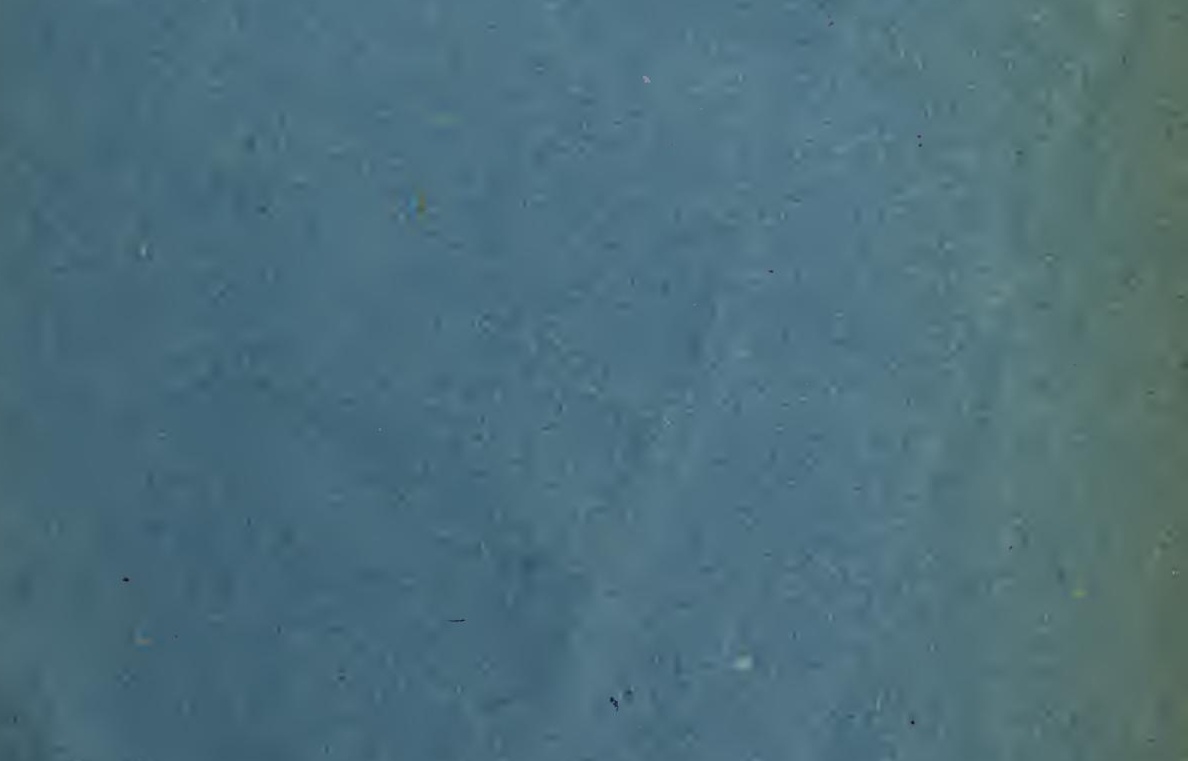

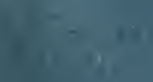

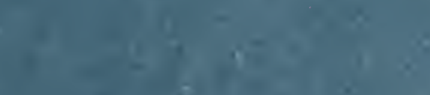




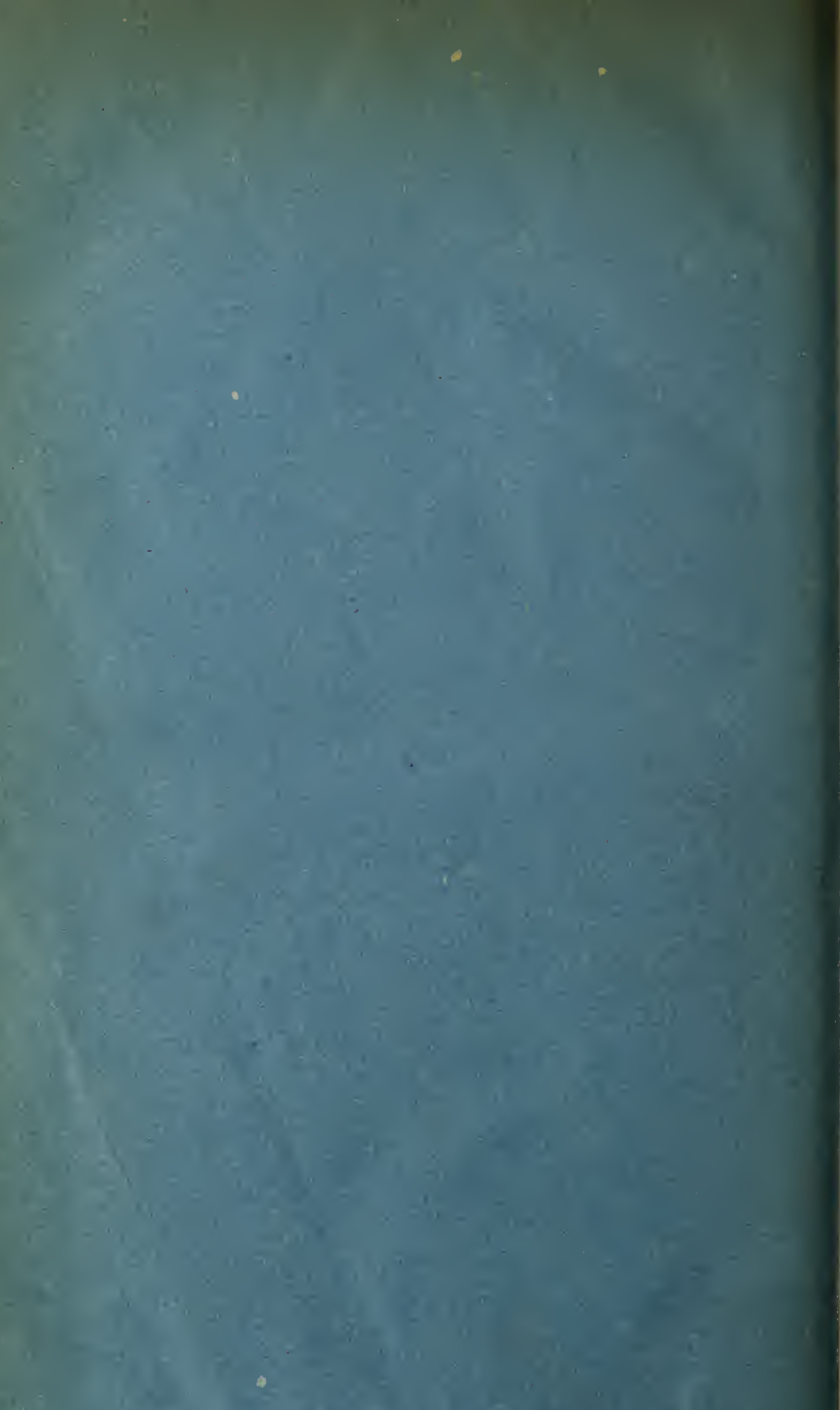





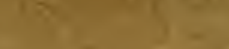

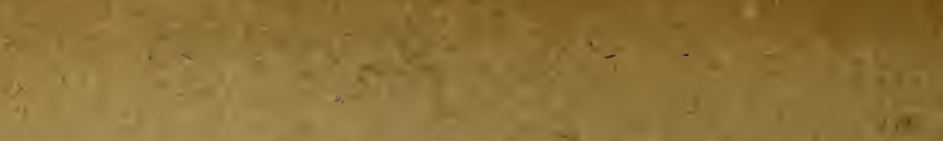

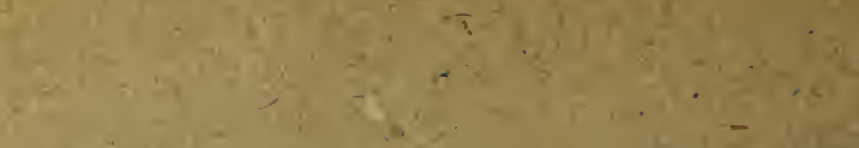

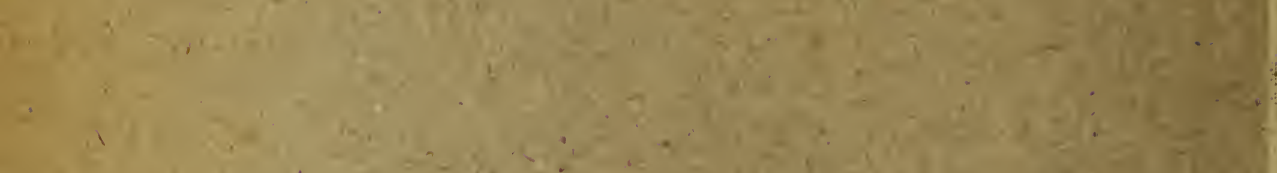
min

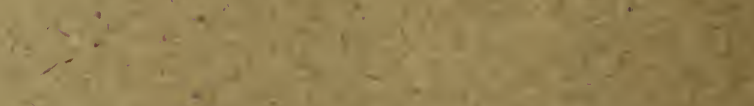

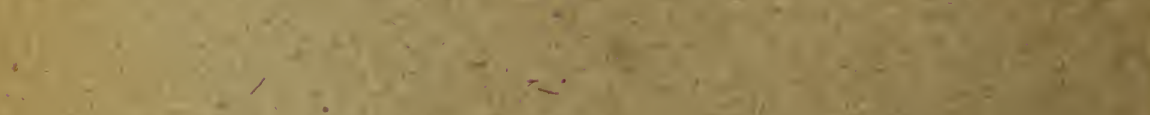

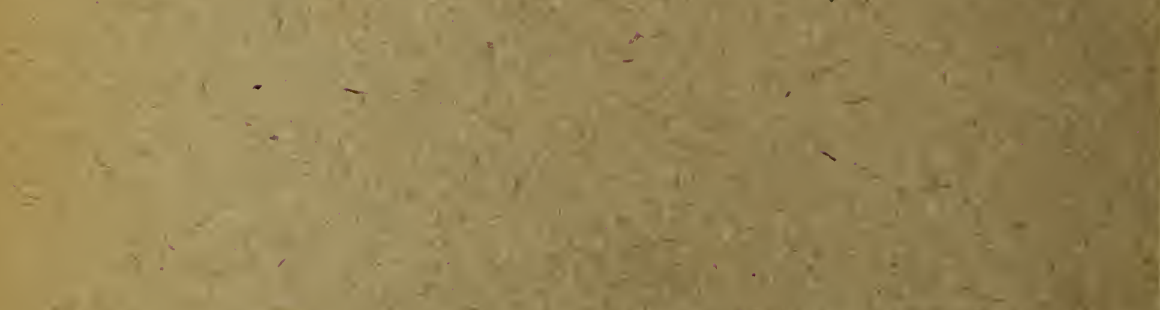

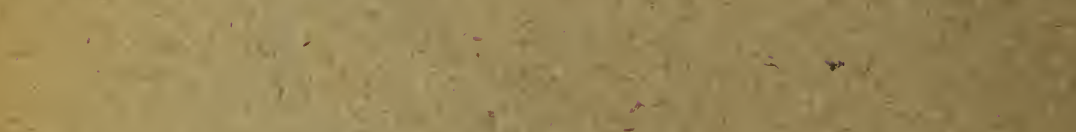

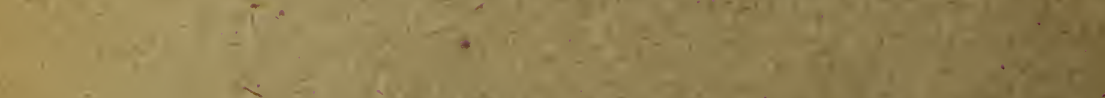

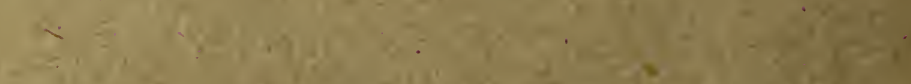

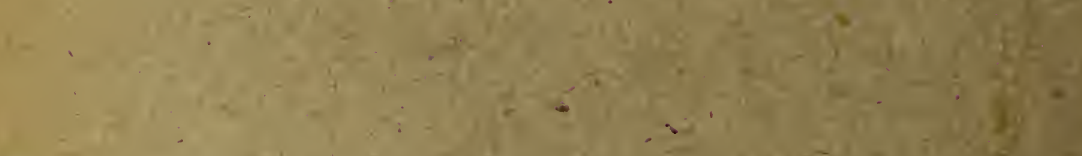

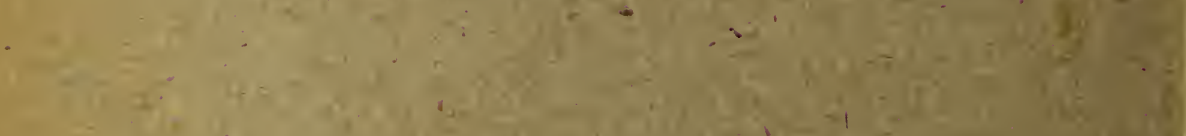

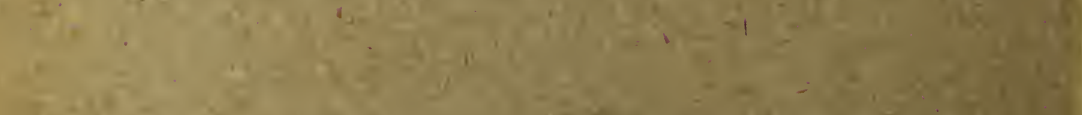

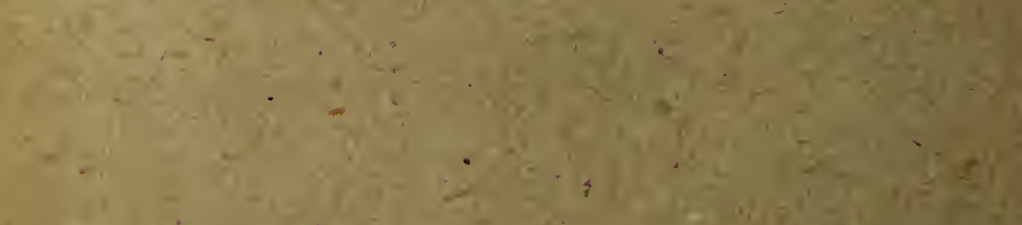

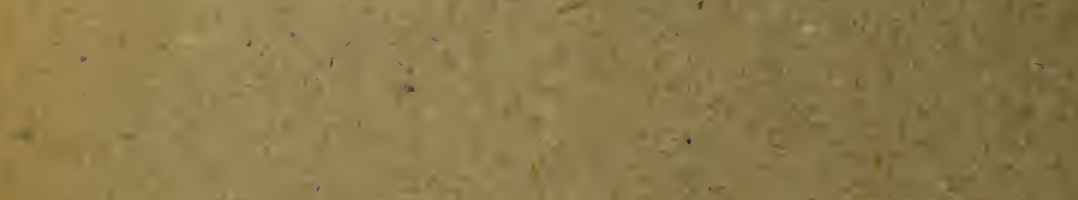

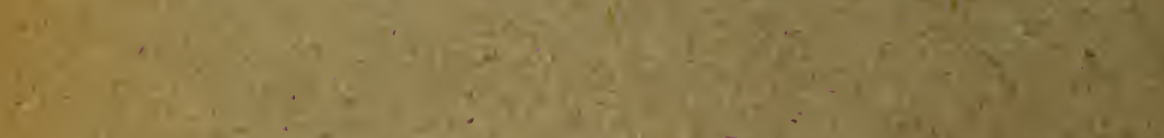

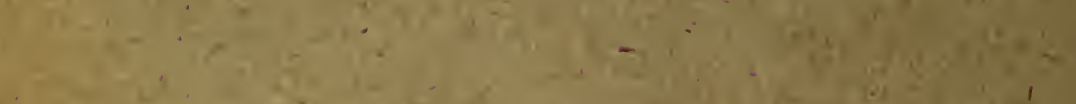

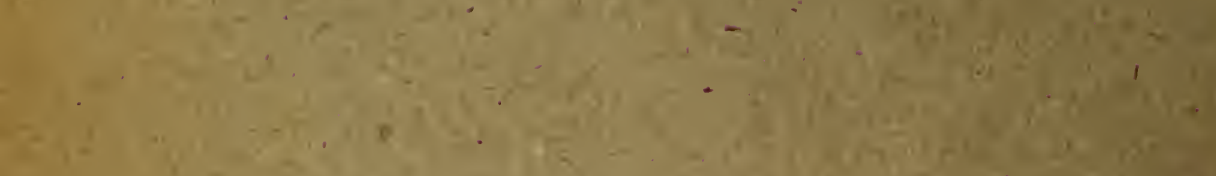

